\title{
Design and Optimisation of Morphing Aircraft
}

\author{
Wang, Chen
}

How to cite:

Wang, Chen (2018) Design and Optimisation of Morphing Aircraft. Doctoral thesis, Swansea University.

http://cronfa.swan.ac.uk/Record/cronfa40876

Use policy:

This item is brought to you by Swansea University. Any person downloading material is agreeing to abide by the terms of the repository licence: copies of full text items may be used or reproduced in any format or medium, without prior permission for personal research or study, educational or non-commercial purposes only. The copyright for any work remains with the original author unless otherwise specified. The full-text must not be sold in any format or medium without the formal permission of the copyright holder. Permission for multiple reproductions should be obtained from the original author.

Authors are personally responsible for adhering to copyright and publisher restrictions when uploading content to the repository.

Please link to the metadata record in the Swansea University repository, Cronfa (link given in the citation reference above.)

http://www.swansea.ac.uk/library/researchsupport/ris-support/ 
Swansea University

Prifysgol Abertawe

\section{Design and Optimisation of Morphing Aircraft}

Chen Wang

Swansea University

March, 2018 
Swansea University

Prifysgol Abertawe

\title{
Design and Optimisation of Morphing Aircraft
}

\author{
by Chen Wang
}

\author{
Supervised by Professor Michael I. Friswell \\ Dr. Hamed Haddad Khodaparast \\ Submitted to Swansea University in fulfilment of the \\ requirements for the Degree of \\ Doctor of Philosophy \\ in \\ Aerospace Engineering
}

Swansea University

March, 2018 


\begin{abstract}
Morphing has the potential to improve the aircraft performance by adaptively changing the shape during different flight conditions. The capabilities of changing shape and carrying aerodynamic loads simultaneously make the design of the morphing structure challenging. The weight increase of morphing aircraft should also be considered, which requires system level analysis and evaluation, and the optimisation of the morphing structure.

The thesis focuses on morphing wingtip devices, which are small in size but have a significant influence on the aerodynamic performance.

A compliant structure based on unsymmetrical stiffness is proposed. The compliant structure has unsymmetrical stiffness allocation, which will have differential axial deflections when it is actuated. The differential deflections lead to a rotation of the compliant structure. A simplified model of the compliant structure is built, and analytical expressions are derived, which highlight the effects of the total stiffness and the stiffness asymmetry. A case study also represents the system level influence of retrofitting a morphing winglet to a baseline wing.

Corrugated panels are applied to provide the stiffness asymmetry. An equivalent model is built to predict the deformation of the corrugated panels. A coupling between the vertical deflection and the axial load is found, which will affect the deflections of the compliant structure significantly. An equivalent beam is used to represent the corrugated panel. The equivalent model is verified by detailed finite element models and experiments.
\end{abstract}

The optimisation of the compliant structure is performed. The actuation force is the objective, while the aerodynamic force and the shape change are included in the optimisation. The influence of the different aerodynamic forces and target shape changes are investigated, which shows the compromise made by the optimised variables to satisfy the constraint and reduce the actuation force. The compliant structures in the earlier case study are optimised, which shows a significant performance increase at the system level.

A demonstration model of the morphing winglet is designed, manufactured and tested. To fit within the thickness of the airfoil, a sequence of optimisations is performed to find the suitable geometry variables. An extreme stiffness asymmetry is required to reduce the actuation force, which validates the proposed morphing structure concept. Static tests and wind tunnel tests are performed to validate the model.

Finally, the contributions of the research are summarised, together with some future work on different aspects.

Key words: morphing aircraft, morphing wingtip devices, morphing winglet, compliant structure, corrugated panels, optimisation 


\section{DECLARATION}

This work has not previously been accepted in substance for any degree and is not being concurrently submitted in candidature for any degree.

Signed (candidate)

Date

\section{STATEMENT 1}

This thesis is the result of my own investigations, except where otherwise stated. Where correction services have been used, the extent and nature of the correction is clearly marked in a footnote(s).

Other sources are acknowledged by footnotes giving explicit references. A bibliography is appended.

Signed (candidate)

Date

\section{STATEMENT 2}

I hereby give consent for my thesis, if accepted, to be available for photocopying and for inter-library loan, and for the title and summary to be made available to outside organisations.

Signed (candidate)

Date 


\section{Acknowledgements}

When the thesis was completed, I had been in Swansea for three and a half years. Swansea University is a good place for my $\mathrm{PhD}$ study, which provides a good research environment and suitable facilities for my research work.

As I am doing my $\mathrm{PhD}$ study in the UK, I would like to thank my parents, who give me their largest support since I was born. I would like to thank my girlfriend, Li Aoxiang. I could not finish the thesis without her understanding and support. A talk with her is a good way to overcome the difficulties in my study and life.

I would like to give my sincere thanks to my supervisor, Prof. Michael Friswell. Prof. Friswell is a very respectful supervisor, who can provide the suitable direction and guide with his rich experience. He is very efficient in his supervision work. He always replies to emails on time and is always willing to give his professional support. I would like to give my sincere thanks to my second supervisor Dr. Hamed Haddad Khodaparast. He provided very useful advice on my $\mathrm{PhD}$ study and is very patient to supervise the progress of my $\mathrm{PhD}$ study. Their excellent supervision has made my PhD study a valuable chance to increase my knowledge and expand my vision. Their supervision helps me to understand that it is more important to find the logical relationship behind the things than just getting the results in the research work. It is such a good choice to work with them, which I would cherish forever.

I would also like to thank Dr. Alexander Shaw, Dr. Ben Woods, Dr. Christopher Beaverstock and Dr. Yuying Xia for their friendly help and support during my $\mathrm{PhD}$ study. Also, I would like to thank the technicians in the College of Engineering for their help in the process of model manufacture and 3D printing.

I would like to give my best wishes to German. He is not only a good group member but a good friend to share a flat. I would also thank Mai, Nidal and Hadi. It is my pleasure to do my $\mathrm{PhD}$ study with them in the same period. Also, I would like to thank my friends Zheng Cui, Yang Chen, Junshen Chen, Yalan Wu and Yakun Yu. The friendly atmosphere helps to accomplish the $\mathrm{PhD}$.

I would like to express my thanks to some friends in China: Chen Wang (his name has the same spelling in English to mine), Jiayi Shu and Yiqun Li. Although we are now in different fields, the common interest in aerospace makes our communication valuable. It is always nice to talk with them.

I would like to thank the China Scholarship Council, and the College of Engineering of Swansea Univerisity, for providing me with the joint scholarship. The scholarship enables my study in the UK. China and the UK have established the close links on the trade and culture despite their significant social and historical differences. If my research work in Swansea Univerisity can make a very tiny contribution to the mutual understanding and communication between the two countries and expand the boundary of knowledge of all human beings, the past three and a half years can be described as 'not wasted'. 
Finally, I would like to cite a famous saying: 'Never forget why you started, then your mission can be accomplished' for my future work and life. 


\section{Contents}

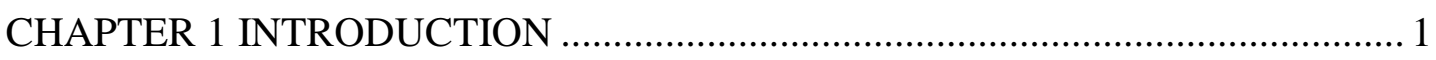

1.1 BRIEF REVIEW OF MORPHING AIRCRAFT ......................................................... 1

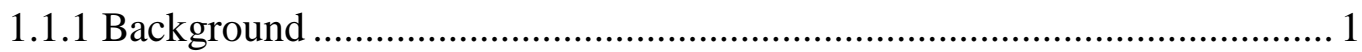

1.1.2 Challenges and opportunities in the development of morphing aircraft ...... 6

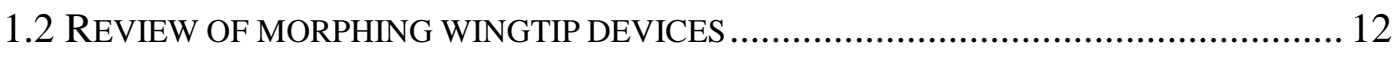

1.2.1 Brief introduction to fixed-geometry wingtip devices ............................. 12

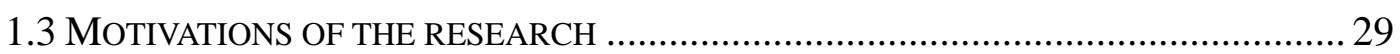

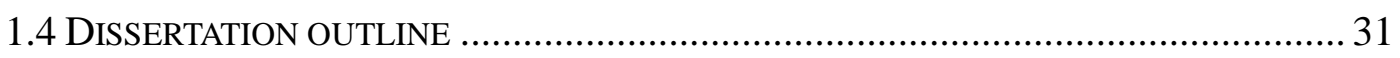

CHAPTER 2 AERODYNAMIC BENEFITS OF MORPHING WINGLETS---A

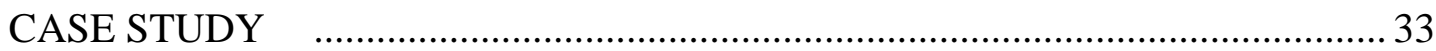

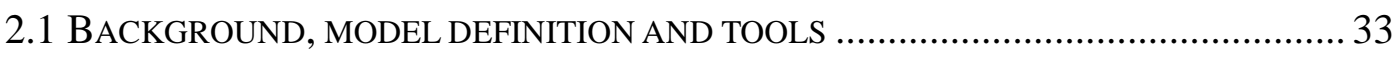

2.2 PARAMETRIC STUDY OF THE MORPHING VARIABLES …................................... 38

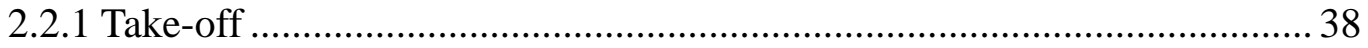

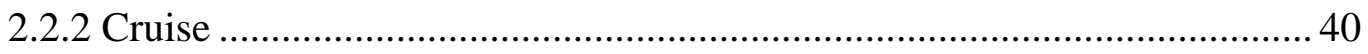

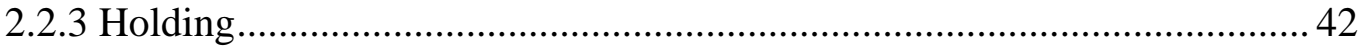

2.3 PoTENTIAL AERODYNAMIC BENEFITS ......................................................... 44

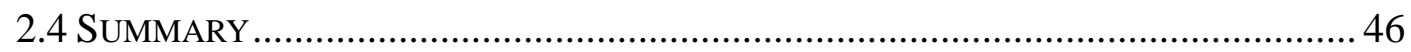

CHAPTER 3 CONCEPTUAL DESIGN OF THE MORPHING WINGLET ........... 47

3.1 COMPLIANT STRUCTURE BASED ON UNSYMMETRICAL STIFFNESS ..................... 47

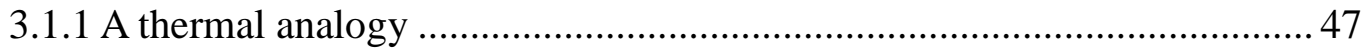

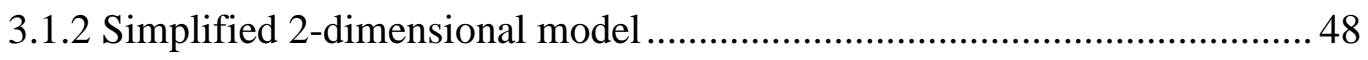

3.2 INFLUENCE OF THE UNSYMMETRICAL STIFFNESS ........................................ 57

3.3 SYSTEM LEVEL BENEFIT DEMONSTRATED IN A CASE STUDY ............................... 63

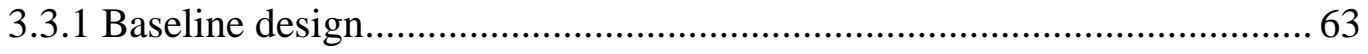

3.3.2 Actuation system design for the weight estimation ...............................6 65

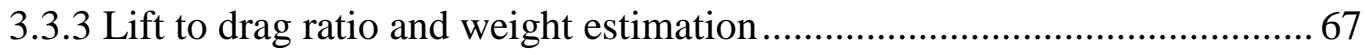

3.4 NUMERICAL SIMULATION OF A LARGE WING MODEL ...................................... 71

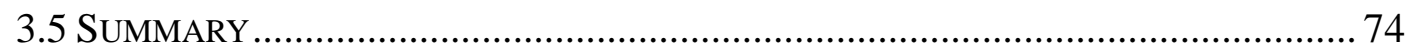

CHAPTER 4 EQUIVALENT MODEL OF CORRUGATED PANELS ................... 77

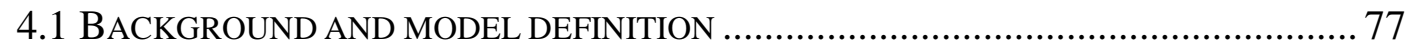


4.2.1 Assumptions and method to calculate the deflections.............................. 80

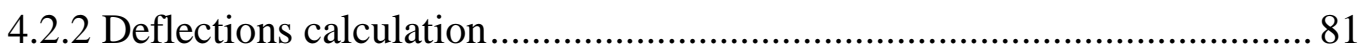

4.2.3 Numerical verification and a modified equivalent model ........................ 86

4.2.4 Method to eliminate the vertical deflection from extension ..................... 92

4.3 EQUIVALENT STIFFNESS MATRIX OF THE CORRUGATED PANELS ......................... 94

4.4 DEFORMATION LIMITS OF CORRUGATED PANELS …......................................... 99

4.5 EXPERIMENTAL VERIFICATION BY TENSILE TEST.............................................. 100

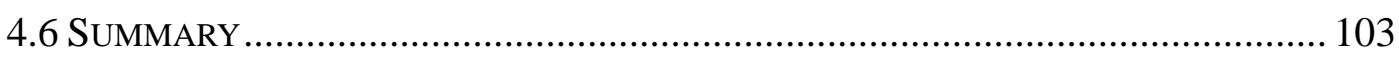

CHAPTER 5 OPTIMISATION OF THE COMPLIANT STRUCTURE USING THE

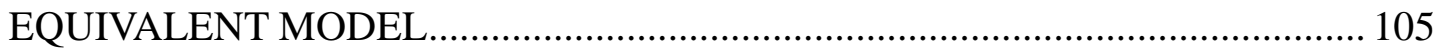

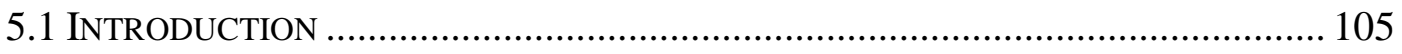

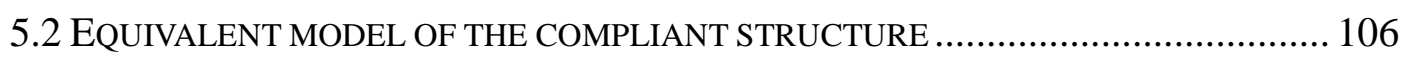

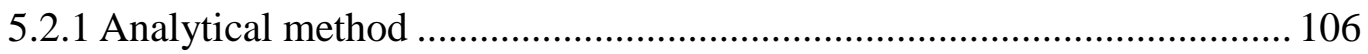

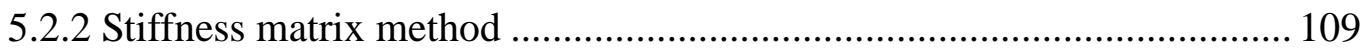

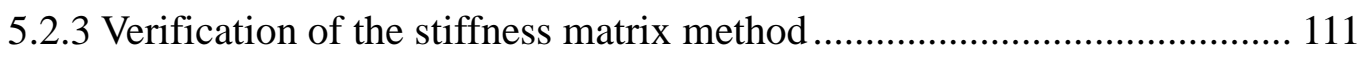

5.4 OPTIMISATION OF A SINGLE COMPLIANT STRUCTURE .................................. 121

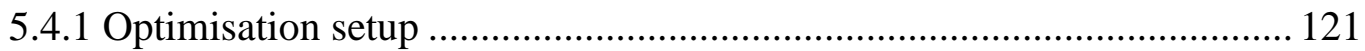

5.4.3 Influence of the geometry constraint................................................. 125

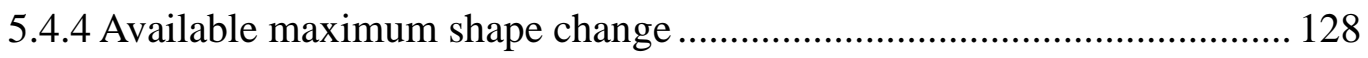

5.5 OPTIMISATION OF MULTIPLE UNITS OF COMPLIANT STRUCTURES ..................... 128

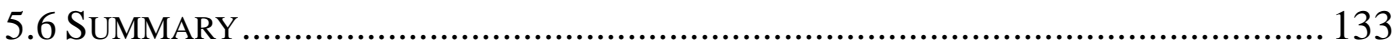

CHAPTER 6 DESIGN AND TEST OF A DEMONSTRATION MODEL .............. 135

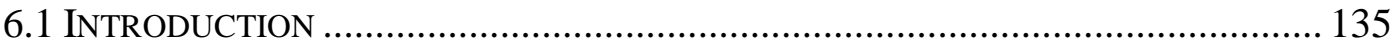

6.2 STRUCTURE INTEGRATION OF THE COMPLIANT STRUCTURE INTO THE WINGLET BY

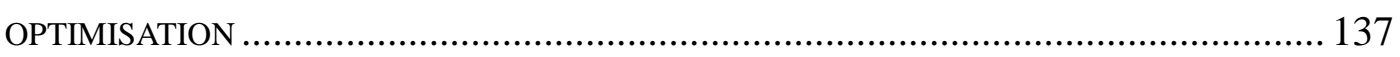

6.2.1 Fitting in the thickness of the airfoil .................................................. 137

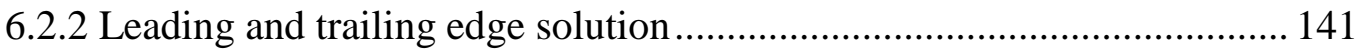

6.2.3 Detailed FEM model verification............................................................ 142

6.3 MODEL MANUFACTURE AND STATIC TESTING ........................................... 143

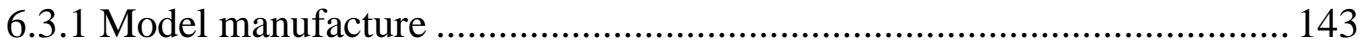

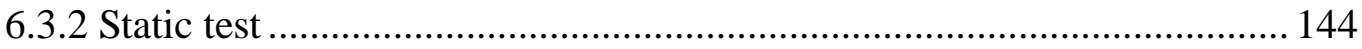

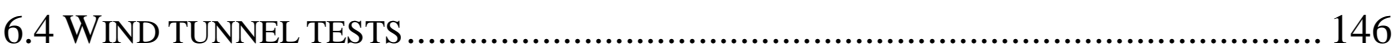




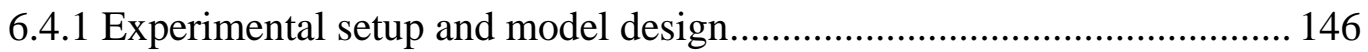

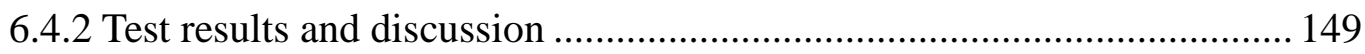

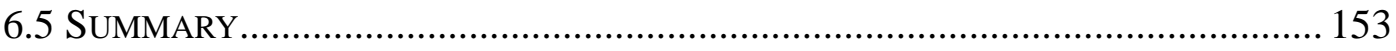

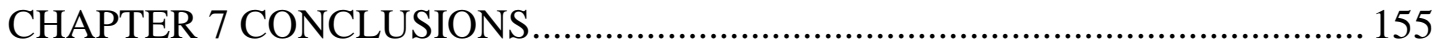

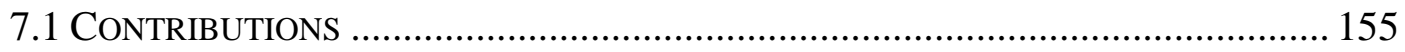

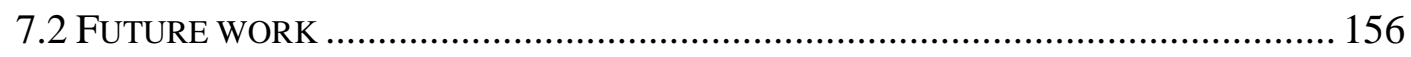

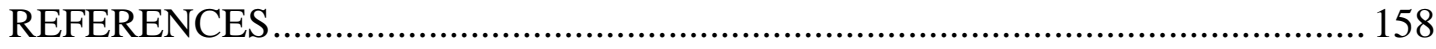




\section{List of figures}

Figure 1.1 (a) Number of publications and (b) Times cited per year in the field of morphing aircraft from the Web of Science $($ C Core Collection database..................... 2 Figure 1.2 (a) Folding wing mechanism from Ader's Avion III (https://www.britannica.com/topic/Ader-Avion-III), (b) Telescope wing of RK-1

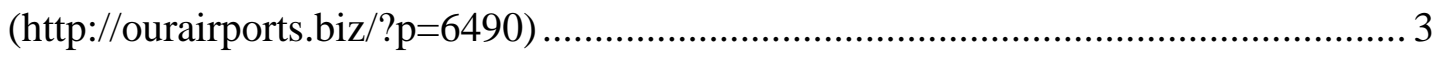

Figure 1. 3 Aircraft platform used in the MAW program ......................................... 4

Figure 1.4 Smart wing model integrated the 'Eccentuator' and SMA actuators [12].. 6 Figure 1.5 Change of the MFX-1 wing platform geometry [13] …......................... 6 Figure 1.6 Requirements triangle illustrating the conflicts in morphing aircraft [23].7 Figure 1.7 Classification of morphing wingtip devices

Figure 1.8 Droop-nose morphing leading edge at the wingtip: (a) Concept, (b) Wind tunnel model [59]

$\begin{array}{llll}\text { Figure } & 1.9 & \text { Active } & \text { winglet }\end{array}$ (http://www.rapp.org/archives/2012/10/aopa-summit/) ......................................... 16

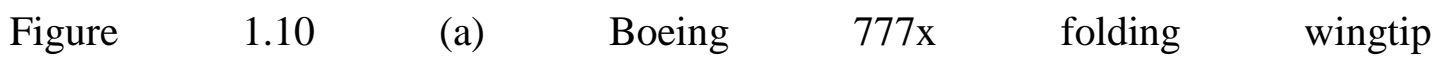
(http://www.boeing.com/commercial/777x/by-design/\#/featured), (b) XB-70 wingtip devices at different angles (http://xb70.interceptor.com/). 17

Figure 1.11 SAW: (a) Un-morphed, (b) Morphed (imaginary) (https://www.nasa.gov/feature/nasa-to-test-in-flight-folding-spanwise-adaptive-wing -to-enhance-aircraft-efficiency)

Figure 1.12 (a) A demonstrator using corrugated skin [66], (b) The partitions of the winglet retrofitted to the fixed wing [68], (c) Optimum dihedral angles of the morphing winglet [68].

Figure 1.13 (a) Unsymmetrical winglet arrangements [74], (b) Two pairs of winglets retrofitted to provide additional control capability [76]

Figure 1.14. (a) Folding wingtip device, (b) Gust excitation test [81] 22

Figure 1.15 (a) Concept of the SMA based soft morphing winglet, (b) Actuation test 
Figure 1.16 (a) Inflatable telescopic winglet [89], (b) auxetic honeycomb structure actuated by pressured tubes [90]

Figure 1.17 Bistable winglet (State 1: high lift configuration, State 2: load alleviation configuration) [92] 26

Figure 1.18 Multiple winglets on an unmanned aerial vehicle to mimic tip feathers of birds [95] 27

Figure 1.19 (a) Control surfaces on the winglet for alleviating wake vortices [98], (b) MFC actuators to oscillate the winglet [99]. 28

Figure 2.1 Sketch of the half wing layout used in Chapter 2 (Geometry parameters taken from [101], [102] ). 34

Figure 2.2 Morphing variables of the winglet 35

Figure 2.3 (a) AVL process step in Matlab (Left), (b) Pre-process example: geometry model in AVL (Right upper), (c) Post-process example: Aerodynamic loads on the wing (Right lower). 37

Figure 2.4 Lift Coefficient vs morphing variables during take-off. 39

Figure 2.5 Normalised wing root bending moment vs morphing variables during take-off 40

Figure 2.6 Lift to drag ratio vs morphing variables during cruise .41

Figure 2.7 Normalised wing root bending moment vs morphing variables during cruise .42

Figure 2.8 Drag coefficient vs morphing variables during holding .43

Figure 2.9 Normalised wing root bending moment vs morphing variables during holding

Figure 3.1 Concept of the compliant structure: (a) Analogy with the bimetallic strip, (b) Rotation of a demonstration sample .48

Figure 3.2 Deflection of the compliant structure: (a) under actuation force, (b) under aerodynamic force 49

Figure 3.3 Loads at point A, D and the geometry relationship of point B, C, and E. 49 Figure 3.4 Internal loads under the actuation force $F$ 
Figure 3.5 Balanced structure and beam $\mathrm{AB}$ and $\mathrm{DC}$

Figure 3.6 Actuated deflection of: (a) Aluminum structure, (b) Composite structure56 Figure 3.7 (a) Geometry of one round corrugation, (b) Equivalent modulus of the round corrugation

Figure 3.8 Rotation angle of the compliant structure represented by the displacement of point E. 59

Figure 3.9 (a) Rotation angle under actuation force and aerodynamic force; (b) Required actuation force for the target rotation angle

Figure 3.10 (a) Rotation angle and (b) required force with larger stiffness asymmetry61

Figure 3.11 Deformation limit of the round corrugation 62

Figure 3.12 Axial strain compared to its limit (a): Upper part, (b): Lower part 63

Figure 3.13 A baseline design with the morphing winglet installed 63

Figure 3.14 (a) Scheme of proposed actuation system, (b) Forces on the mechanism with two worms .66

Figure 3.15 Estimation of weight change 68

Figure 3.16 (a) Relative increase of lift to drag ratio and weight, (b) Required number of actuation sets .70

Figure 3.17 Structure and loads of a large wing model .72

Figure 3.18 (a)Winglet angle, (b) Normalised lift change over the analysis steps .... 73

Figure 3.19 Deformation and stress distribution of the wing. 74

Figure 4.1 (a) Trapezoidal corrugation panel, (b) Round corrugation panel 79

Figure 4.2 Geometry of the corrugation unit: (a) trapezoidal profile, (b) round profile79

Figure 4.3 Schematic of the deflection components .80

Figure 4.4 Geometry and bending moment in the corrugation unit: (a): trapezoidal, (b): round, (c): separate beams in the round unit 81

Figure 4.5 Equivalent axial modulus of the trapezoidal corrugation panel 87

Figure 4.6 Equivalent axial modulus of the round corrugation panel. .88

Figure 4.7 Influence of the beam extension on the equivalent axial modulus..... 89

Figure 4.8 Vertical deflections verified by the FEM model, (a): Trapezoidal, (b):

Round corrugation 90 
Figure 4.9 Vertical deflection under the pure extension load.

Figure 4.10 Offset boundary condition and the vertical deflection caused by the opposite rotation .94

Figure 4.11 Equivalent element representing the corrugated panel .94

Figure 4.12 Internal loads of the trapezoidal corrugated panel under $X$ and $Y$ .96

Figure 4.13 Internal loads of the round corrugated panel under $X$ and $Y$. .99

Figure 4.14 Geometry of the test samples. 101

Figure 4.15 (a) Tensile test of the corrugated panel; (b) Nondimensionalized tensile modulus validation. 102

Figure 5.1 Geometry relationship in the compliant structure 106 Figure 5.2 Vertical deflections of the compliant structure calculated by the analytical method 108

Figure 5.3 Equivalent model of the compliant structure; (b) Equivalent beam element representing the corrugated panel 109

Figure 5.4 Round corrugation: (a) Mesh of the finite element model, (b) An example of the vertical deflections obtained 112 Figure 5.5 Verification of the compliant structure equivalent model by detailed finite element analysis: case $1\left(a=0.12 \mathrm{~m}, b=0.1 \mathrm{~m}, w=0.01 \mathrm{~m},{ }^{1} R=0.01 \mathrm{~m},{ }^{1} L=0.015 \mathrm{~m}\right.$, $\left.{ }^{2} R=0.01 \mathrm{~m},{ }^{2} L=0.015 \mathrm{~m},{ }^{1} t=0.002 \mathrm{~m},{ }^{1} n=3,{ }^{2} n=3\right)$.

Figure 5.6 Verification of the compliant structure equivalent model by detailed finite element analysis: case $2\left(a=0.16 \mathrm{~m}, b=0.2 \mathrm{~m}, w=0.01 \mathrm{~m},{ }^{1} R=0.02 \mathrm{~m},{ }^{1} L=0.02 \mathrm{~m}\right.$, $\left.{ }^{2} R=0.02 \mathrm{~m},{ }^{2} L=0.02 \mathrm{~m},{ }^{1} t=0.002 \mathrm{~m},{ }^{1} n=2,{ }^{2} n=2\right)$

Figure 5.7 Verification of the compliant structure equivalent model by detailed finite element analysis: case 3(trapezoidal corrugated panels: $a=0.204 \mathrm{~m}, b=0.08 \mathrm{~m}$, $w=0.01 \mathrm{~m},{ }^{1} l_{1}=0.015 \mathrm{~m},{ }^{1} l_{2}=0.025 \mathrm{~m},{ }^{1} \theta=30{ }^{\circ},{ }^{1} t=0.001 \mathrm{~m}^{2} l_{1}=0.015 \mathrm{~m},{ }^{2} l_{2}=0.025 \mathrm{~m}$, $\left.{ }^{2} \theta=30^{\circ},{ }^{1} n=2,{ }^{2} n=2\right)$

Figure 5.8 A compliant structure based on stiffness asymmetry..... 115

Figure 5.9 (a) Deflections of the sample under actuation; (b) Comparison of the slopes between the lateral and axial deflections 116 Figure 5.10 (a) Actuation location (black arrow), un-deformed structure(blue lines) 
and deformed structure (red lines), (b) Nondimensionalized deflections under different actuation locations

Figure 5.11 Normalised rotation angle with changed height $b$ under (a) actuation

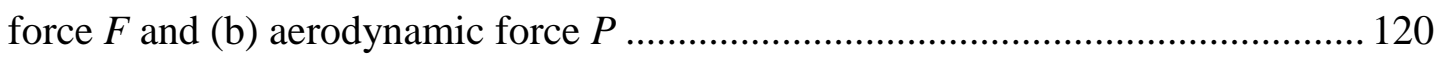

Figure 5.12 Multiple units of compliant structures .................................................. 121

Figure 5.13 Optimisation variables of compliant structures made of on (a) round, (b) trapezoidal corrugated panels

Figure 5.14 (a) Optimised actuation force vs required rotation angle, (b) Optimised thickness ${ }^{2} t$ vs aerodynamic force

Figure 5.15 Influence of height b on (a) optimised actuation $F$, (b) geometry variable ${ }^{1} L$ and the sum of ${ }^{1} L+{ }^{1} R+{ }^{2} L+{ }^{2} R$. 125

Figure 5.16 (a) Wing span extension via the morphing winglet, (b) Conceptual design of the morphing winglet

Figure 5.17 Optimisation procedure of the multiple units of the compliant structure131 Figure 5.18 (a) Sum of the optimised actuation forces with a fixed length $E S$, (b) Optimised weight increase compared to the results a fixed geometry

Figure 6.1 The morphing winglet demonstrator (The wingtip fairing and elastomer skin are not included) 136

Figure 6.2 (a) Optimised height and actuation force, (b) Compliant structure in the airfoil.

Figure 6.3 (a) Compliant structure based on corrugated panels, (b) Flexible honeycomb structure 140

Figure 6.4 (a) Abaqus model used for numerical verification, (b) von Mises stress distribution in the deformed structure

Figure 6.5 Static test demonstration when the actuator position varies from $9 \%$ to $29 \%$ of the stroke

Figure 6.6 Wind tunnel test setup and the model installation 147

Figure 6.7 Components and assembly of the wind tunnel model 148

Figure 6.8 Influence of the initial dihedral angle on the (a) Lift coefficient and (b) Rolling moment coefficient. 149 
Figure 6.9 Comparisons of the wind tunnel test results: (a) Lift coefficient, (b) Drag coefficient... 150

Figure 6.10 The wind tunnel model: (a) Front view, and (b) Side view with different the actuator extensions 151

Figure 6.11 Change of (a) lift coefficient and (b) rolling moment coefficient caused by the morphing wingtip 152 


\section{List of tables}

Table 2.1 Main parameters of the aircraft in the case study..................................... 34

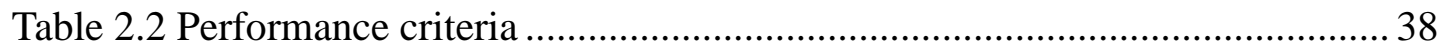

Table 2.3 Selected morphing variables during different phases.............................. 45

Table 2.4 Morphing winglet performance compared to a fixed winglet................... 45

Table 3.1 Properties of the matrix and fibre........................................................... 55

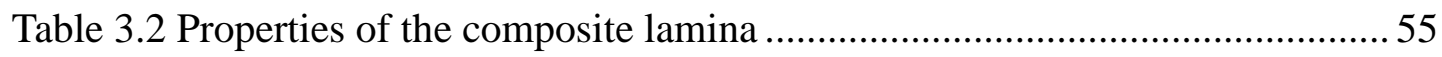

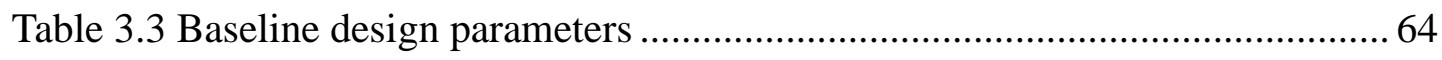

Table 4.1 Axial deflections of the points in the corrugation unit ............................... 84

Table 4.2 Rotation of the beam cross-section and vertical deflections in the

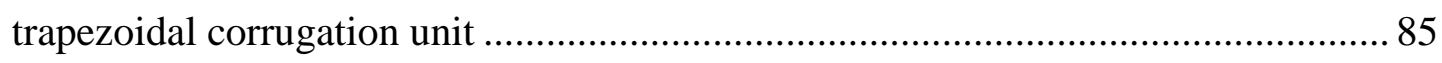

Table 4.3 Rotation of the beam cross-section and vertical deflections in the round

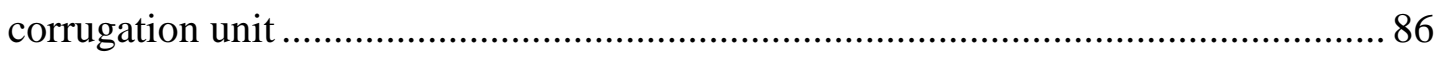

Table 5.1 Geometry parameters of the round corrugation samples ........................ 116

Table 5.2 geometry parameters in the parametric analysis cases ........................... 118

Table 5.3 Optimised variables of the compliant structure. Note that the optimised

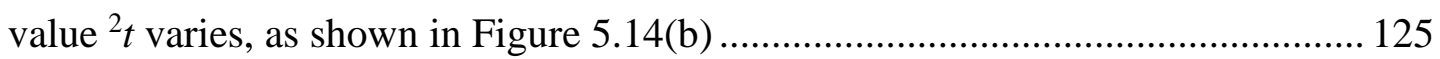

Table 5.4 Optimised geometry variables when the height $b$ varies. Note that the

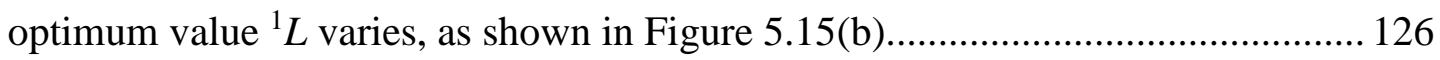

Table 5.5 Optimisation results of the upper panel with changing height $b$............. 127

Table 5.6 Optimisation results of the lower panel with changing height $b$............. 127

Table 5.7 Maximum rotation angle of the compliant structure .............................. 128

Table 6.1 Optimised variables and properties of the compliant structure in the

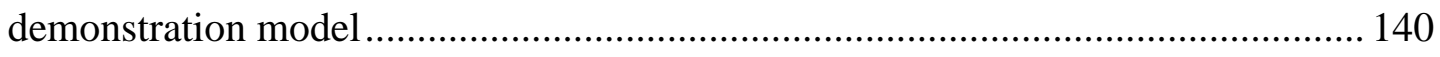

Table 6.2 Selected variables of the flexible honeycomb structures ....................... 142

Table 6.3 Force and moment capacity of the balance ............................................ 146 


\section{Nomenclature}

\section{Symbols}

$\begin{array}{cc}\theta_{d} & \text { winglet dihedral angle } \\ \theta_{t} & \text { winglet toe angle } \\ \theta_{w} & \text { winglet twist angle } \\ \theta_{c} & \text { deflection angle of the winglet control surface } \\ C_{L} & \text { lift coefficient } \\ C_{D} & \text { drag coefficient } \\ C_{M} & \text { rolling moment coefficient } \\ L / D & \text { lift to drag ratio }\end{array}$

$L \quad$ lift in Chapter 2, otherwise straight length of round corrugation

$D$

$T_{h r}$

$S_{a}$

$R_{a}$

$R_{c}$

$R_{t}$

$h_{s c}$

$\gamma_{c}$

$W$

$W_{i}$

$W_{f}$

$\Delta W_{s}$

$\Delta W_{a}$

$\Delta W_{e}$

$W_{\text {wing }}$

$W_{d g}$

$m$

drag

thrust

take-off transition distance

range of the aircraft

radius during the climbing phase

turn radius of aircraft

screen height during take-off

climbing angle

aircraft weight

initial aircraft weight

final aircraft weight

weight change due to the geometry change

weight of the installed actuation system

weight change due to the retrofitted morphing winglet

wing weight

design gross weight of the baseline model

aircraft mass 


\begin{tabular}{|c|c|}
\hline$\stackrel{\square}{W}$ & time rate of the change of aircraft weight \\
\hline$N_{z}$ & ultimate load factor \\
\hline$S_{w}$ & wing area \\
\hline$S$ & wing span \\
\hline$(t / c)_{\text {root }}$ & airfoil thickness ratio at the wing root \\
\hline$\Lambda$ & sweep angle of the $25 \%$ of mean aerodynamic chord \\
\hline$\lambda$ & wing taper ratio \\
\hline$A_{w}$ & wing aspect ratio \\
\hline$S_{c s w}$ & control surface area of the wing \\
\hline$\rho$ & air density \\
\hline$g$ & gravity acceleration \\
\hline$V$ & flight speed \\
\hline$C$ & specific fuel consumption \\
\hline$\beta_{b}$ & bank angle during the steady banked flight \\
\hline$a$ & length of the compliant structure \\
\hline$b$ & height of the compliant structure \\
\hline$w$ & width of the compliant structure \\
\hline$B$ & entire height of the compliant structure \\
\hline$E$ & Young's modulus \\
\hline$A$ & cross section area \\
\hline$I$ & second moment of area \\
\hline$\overline{E A}$ & equivalent property related to the tension stiffness \\
\hline$\overline{E I}$ & equivalent property related to the bending stiffness \\
\hline$K_{c}$ & coupling between the axial force and vertical deflection \\
\hline$P$ & aerodynamic force \\
\hline$F$ & actuation force \\
\hline$X$ & force in the $x$-direction \\
\hline$Y$ & force in the $y$-direction \\
\hline
\end{tabular}




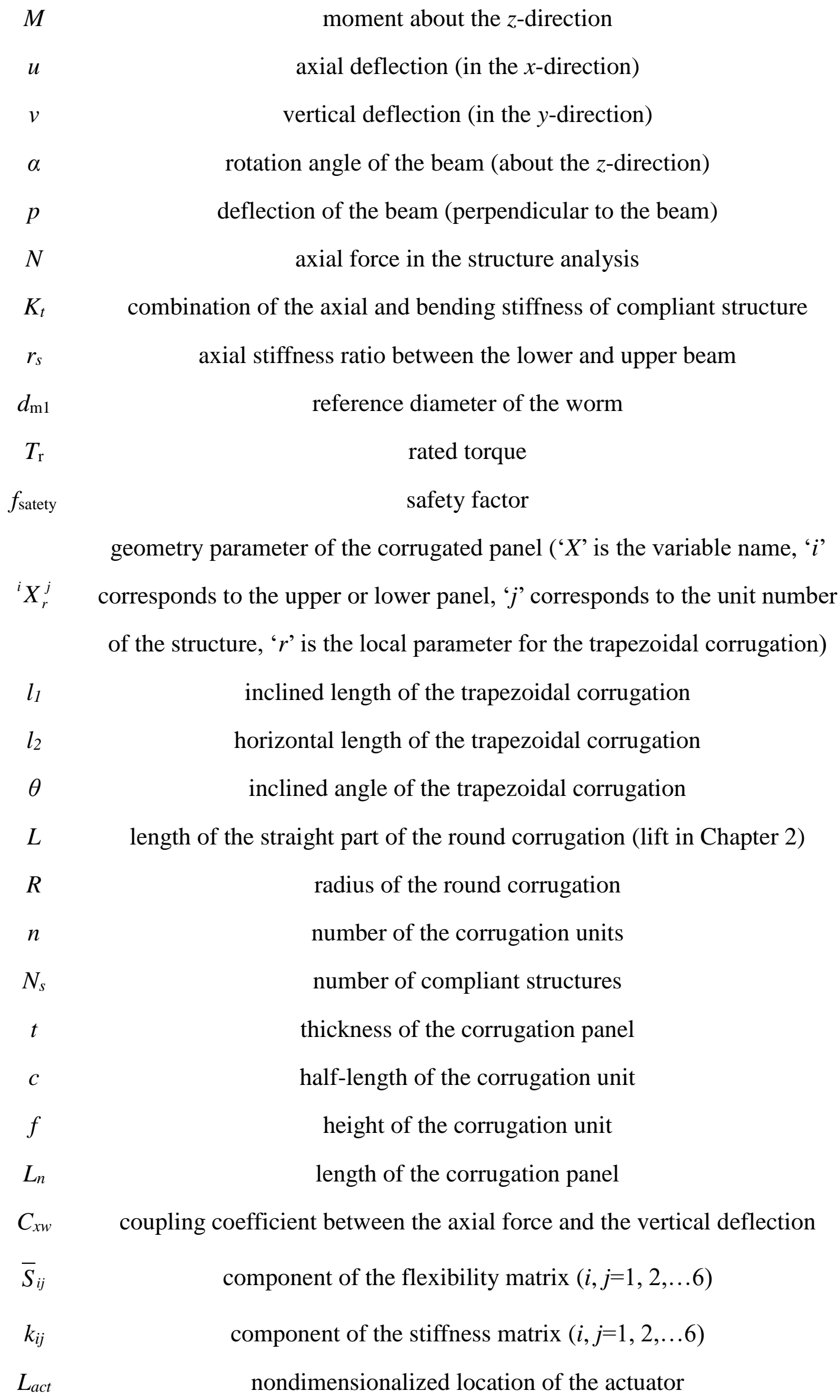




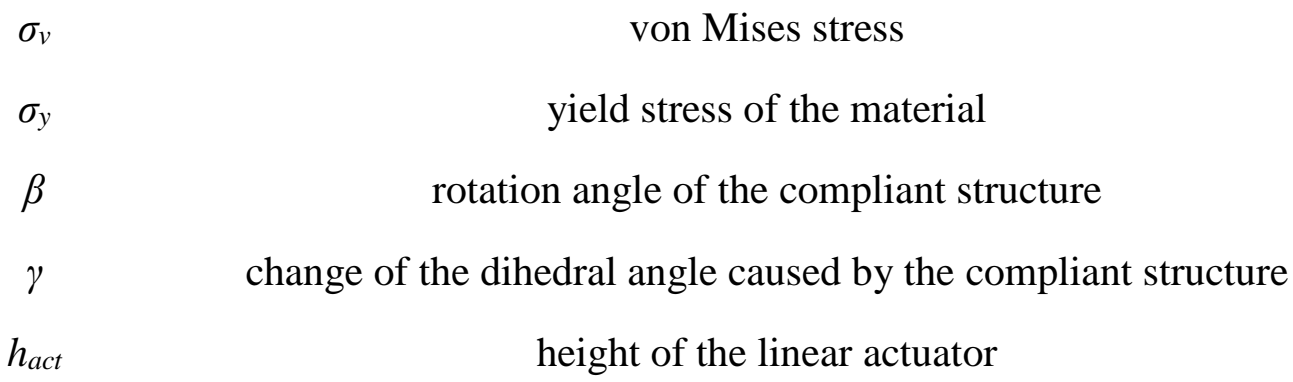

Abbreviations

AoA

Angle of Attack

VLM Vortex Lattice Method

FEM Finite Element Method

SMA Shape Memory Alloy

MFC Macro Fiber Composite

FishBAC Fish Bone Active Camber Concept

MAW Mission Adaptive Wing

MAS Morphing Aircraft Structures

VGC Variable Geometry Chevron 


\section{List of publications}

1. Wang, C., Haddad Khodaparast, H., and Friswell, M.I. Investigating the benefits of morphing wingtip devices---A case study. International Forum on Aeroelasticity and Structure Dynamics. Saint Petersburg, Russia, June 28-July 02, 2015.

2. Wang, C., Haddad Khodaparast, H., and Friswell, M.I., Conceptual study of a morphing winglet based on unsymmetrical stiffness. Aerospace Science and Technology, 2016. 58: p. 546-558.

3. Wang, C., Haddad Khodaparast, H., Friswell, M.I., and Shaw, A.D., An equivalent model of corrugated panels with axial and bending coupling. Computers \& Structures, 2017. 183: p. 61-72.

4. Wang, C., Haddad Khodaparast, H., Friswell, M.I., and Shaw, A.D., Compliant structures based on stiffness asymmetry. The Aeronautical Journal, 2018. 122(1249): p. $442-461$.

5. Wang, C., Friswell, M.I., Haddad Khodaparast, H., and Shaw, A.D. Morphing winglets based on compliant structures. VIII ECCOMAS Thematic Conference on Smart Structures and Materials SMART 2017. Madrid, Spain, June 5-8, 2017.

6. Wang, C., Friswell, M.I., Haddad Khodaparast, H., Shaw, A.D., Xia, Y., and Walters, P., Development of a Morphing Wingtip Based on Compliant Structures. Journal of Intelligent Material Systems and Structures (Accepted) 


\section{Chapter 1 Introduction}

\subsection{Brief review of morphing aircraft}

\subsubsection{Background}

Morphing aircraft, which have the capability of shape-changing during flight to improve their performance for different flight flights, have become a promising candidate for the future aircraft technology. Conventionally, the aircraft is designed with a fixed geometry, even though the geometry is optimised. However, the performance of the aircraft can only be optimal for some fixed flight conditions, which limits its capability to perform diverse flight missions.

Weisshaar [1] described morphing aircraft with three phrases: 'multi-regime', 'aerodynamically-efficient' and 'shape-changing'. Morphing aircraft are expected to perform multi-role missions with a supreme performance by adopting morphing. Jha and Kudva [2] summarised the morphing concepts and classified the morphing designs based on the geometry parameter that was changed, which gave a straightforward way to understand morphing aircraft. A comprehensive review of morphing aircraft was reported in [3], in which the focus was concentrated on wing morphing. The morphing wing was classified into three types: 'planform', 'out-of-plane' and 'airfoil'. More examples using smart materials, such as piezoelectric material and shape memory alloy, were introduced, which showed a technology push behind the development of morphing aircraft.

From the perspective of the author, the main feature of morphing aircraft is the capability to adapt the aircraft shape to the flight condition. The shape change can be either large or small in comparison to the size of the aircraft, while the shape-changing should be able to generate a relatively large impact on the performance of the aircraft, considering the potential (weight) penalty caused by the 
morphing structures and actuators.

The subject of morphing aircraft has been intensively researched in the recent years. Figure 1.1 shows the number of publications on morphing aircraft from Web of Science core collection [4], which only represents part of the research publications in this field. The number of publications and times cited per year have increased continuously in the past 15 years, indicating the increased attention paid to this field.

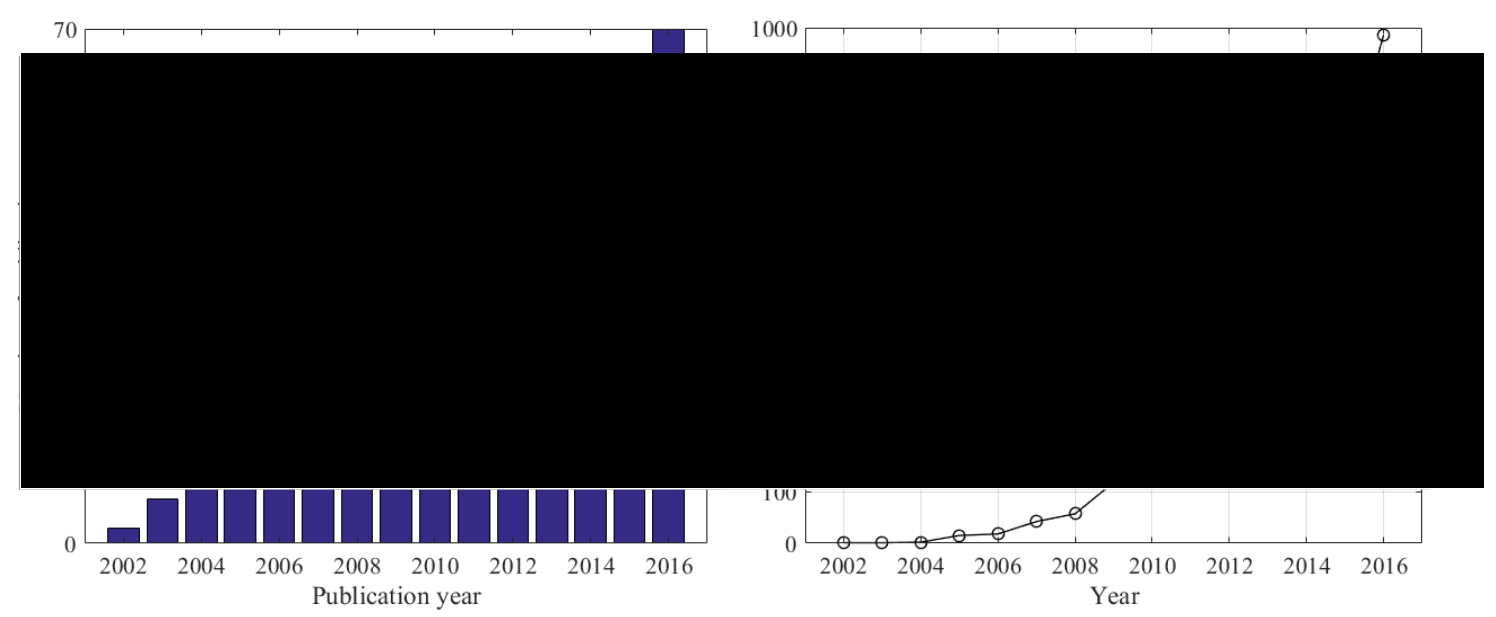

Figure 1.1 (a) Number of publications and (b) Times cited per year in the field of morphing aircraft from the Web of Science $($ C Core Collection database

Although the phrase 'morphing aircraft' has generated an increased interest in recent years, the concept of the shape-changing aircraft can be traced back to the dawn of the aviation age. For instance, the Avion III, designed by Clement Ader, has a wing structure like that of a bat and seems to be able to unfold itself for the flight according to its pictures [5] as shown in Figure 1.2(a), despite the lack of the flight proof. The Wright brothers, who are believed to have performed the first powered flight with a heavier-than-air machine successfully, adopted a flexible wing-warping mechanism for roll control, which played a significant role in their successful flights [6]. The wing warping was used to provide roll control in the early days of aviation until they were replaced by ailerons. In the 1930s, the USSR designer Bakshayev designed the RK-1 with a telescopic wing consisted of 6 pieces of wood as shown in Figure 1.2(b). The wing area could be increased during take-off and landing to reduce the take-off/landing distance by extending the telescopic wing from the 
fuselage, while the telescopic wing could be retracted by the pilot when a high flight speed was required. A more ambitious program was aimed at achieving a flight speed of $800 \mathrm{~km} / \mathrm{h}$, with a more dramatic wing area change and powerful engine, but unfortunately the program was stopped in the 1940s [7].

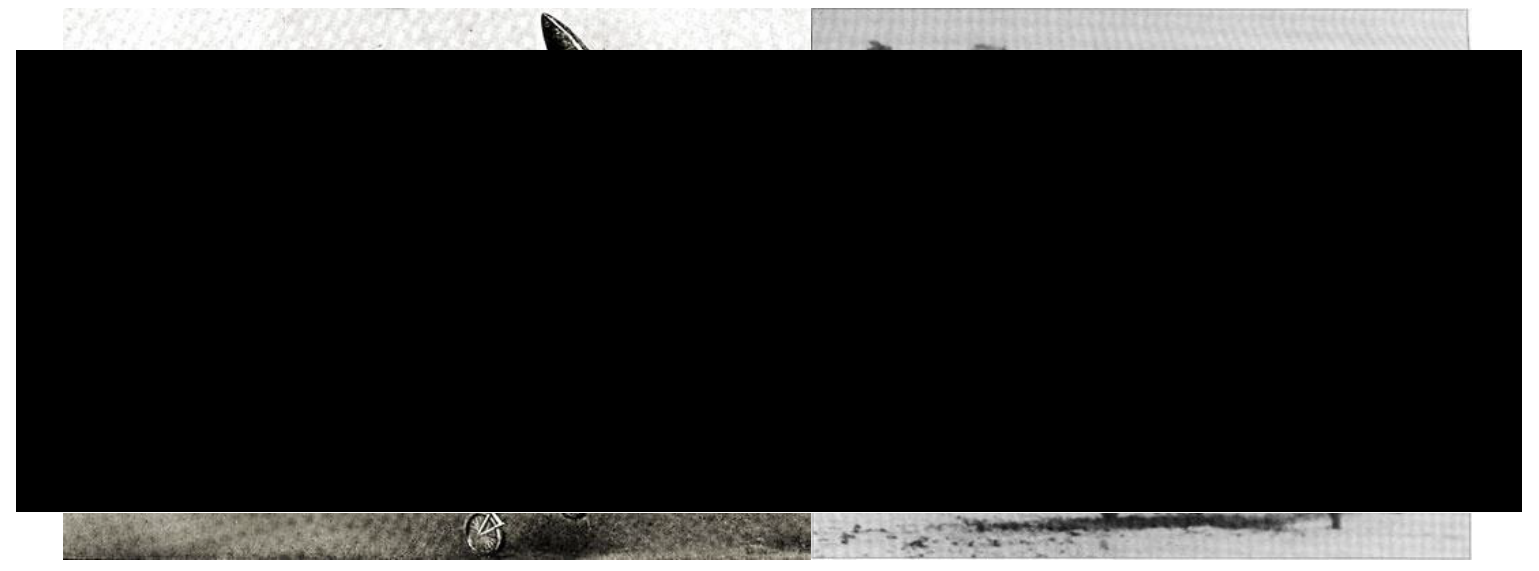

Figure 1.2 (a) Folding wing mechanism from Ader's Avion III (https://www.britannica.com/topic/Ader-Avion-III), (b) Telescope wing of RK-1 (http://ourairports.biz/?p=6490)

The increased force and energy requirements of the actuation system make the aircraft shape-changing to be ineffective due to the unacceptable weight penalty and system complexity. However, the rapid development of the new materials, actuators, computers and design methods has made the concept of morphing aircraft promising since the 1980s.

In the 1980s, a smooth variable camber morphing wing was developed in the Mission Adaptive Wing (MAW) program. The camber morphing wing was equipped with both morphing leading and trailing edges, which could be used to optimise the airfoil shape for different flight conditions. The MAW was also able to provide roll control, manoeuvre load control and gust load alleviation [8]. The design of the flexible trailing edge structure was shown in the literature, which was based on an internal hinged mechanism and hydraulic actuators [9]. Using the wind tunnel data stored in the flight computer, the flight control system allowed the automatic deflection of the leading and trailing edges, and the deflection angle depended on the Mach number, dynamic pressure and even wing sweep [10]. A camber morphing 
wing was tested on an F-111 aircraft as shown in Figure 1.3, and the aircraft was capable of wing planform morphing (variable sweep wing) and airfoil morphing. The capability of the MAW was shown by ground and flight tests, although one drawback of the morphing was the complicated internal mechanism, which led to a large weight compared to conventional leading and trailing edges, and more challenges in the manufacturing and operation. It should also be noted that in the literature of the time the morphing wing was named the 'adaptive wing'.

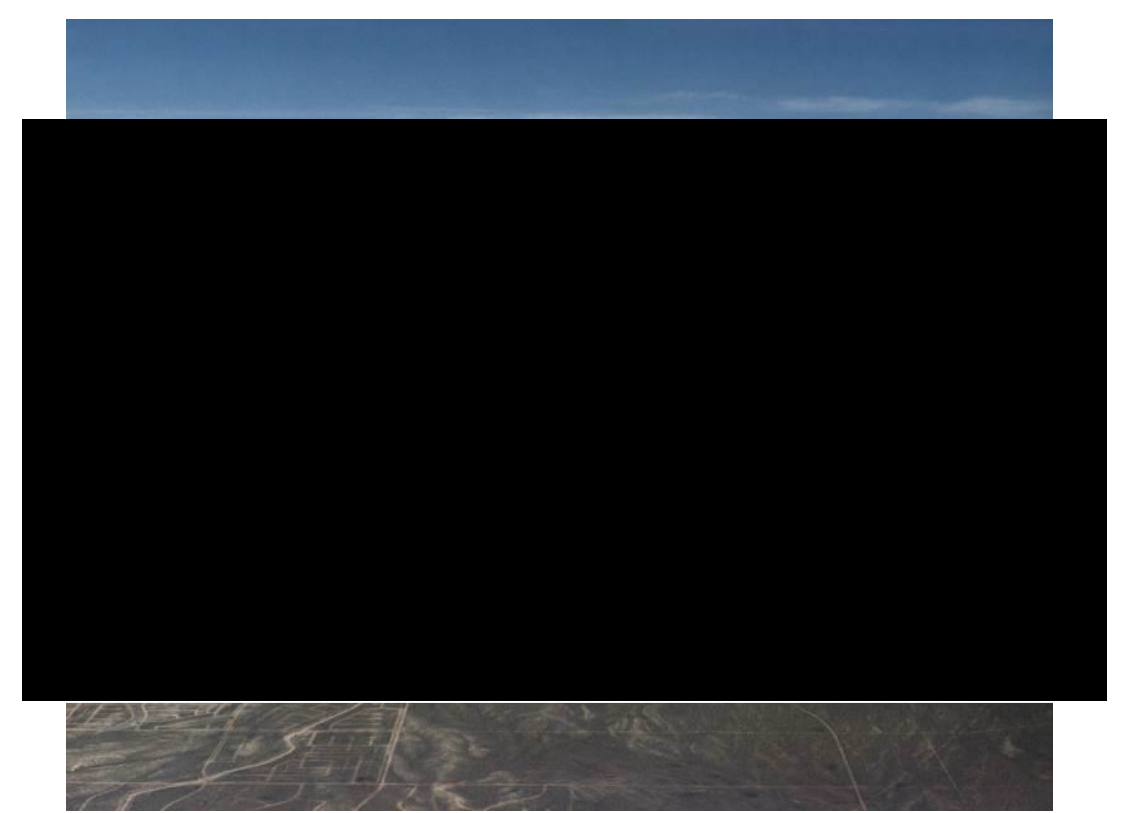

Figure 1. 3 Aircraft platform used in the MAW program (https://www.nasa.gov/centers/dryden/multimedia/imagegallery/F-111AFTI/EC85-3 3205-07.html)

The MAW program showed promising performance benefits by deflecting the leading and trailing smoothly. In the 1990s, some innovative actuation solutions were investigated in the Smart Wing program, which could provide more morphing benefits by reducing the complexity and the weight increase. Two review papers summarised the research work in the Smart Wing program for Phase I [11] and Phase II [12] respectively. The Shape Memory Alloy (SMA) actuator was designed and tested in Phase I; the actuator was based on a TiNi tube and was able to provide a torque as large as 3000 in-lbs. The structural connection of the SMA tube was carefully designed to ensure that the actuation force generated by the SMA actuator 
could be transmitted to the structure, especially when the actuator was integrated into the wing structure. The test was performed on a scaled wing structure model, which was able to introduce over 5 degrees of wing tip twist and deflect the trailing edge at a similar level to the aileron but with a higher rolling moment. The aerodynamic investigation of the smooth trailing edge deflection also suggested a performance increase compared to the conventional control surface. Although the SMA actuator could generate a large actuation force, the cooling process was inherently slow, limiting the bandwidth to one or two Hertz, which made the SMA actuator not suitable for active manoeuvre control. In Phase II of the program, an ultrasonic piezoelectric motor was used to reduce the response time of the actuation system. Figure 1.4 shows the full-span unmanned aerial vehicle model that was employed in this phase, which required 60-70 degrees of deflection of the control surface per second. The actuation force was transmitted into the structure with a so-called 'eccentuator', which was a bent beam connected to trailing edge. More than one eccentuator could be used to change the twist of the wing. Compliant structures, such as flexible honeycomb structures and flexible skin panels, were also essential for the successful development of the experimental model. More direct performance improvements were reported with comparisons to conventional designs, and the results obtained contributed to 'a strong foundation' for the potential applications of morphing aircraft.

The MFX-1 developed by NextGen Aeronautics was another milestone in the development of morphing aircraft, which demonstrated a successful in-flight, large-scale morphing [13]. The MFX-1 was developed under the NextGen Morphing Aircraft Structures (N-MAS) program and aimed at providing substantial geometry change, e.g. $200 \%$ change in the aspect ratio and $70 \%$ change in the wing area [14], as shown in Figure 1.5. The massive geometry change was supported by some core morphing technologies [15], such as the flexible skin, which could undergo in-plane shearing deformation, and the wing mechanism, which should carry the loads and change the wing geometry simultaneously. The actuation system, which had to 
provide enough actuation force and satisfy the weight and installation constraints, was another critical aspect. A multi-disciplinary optimisation of the distributed actuation system was developed to solve the problem [16].

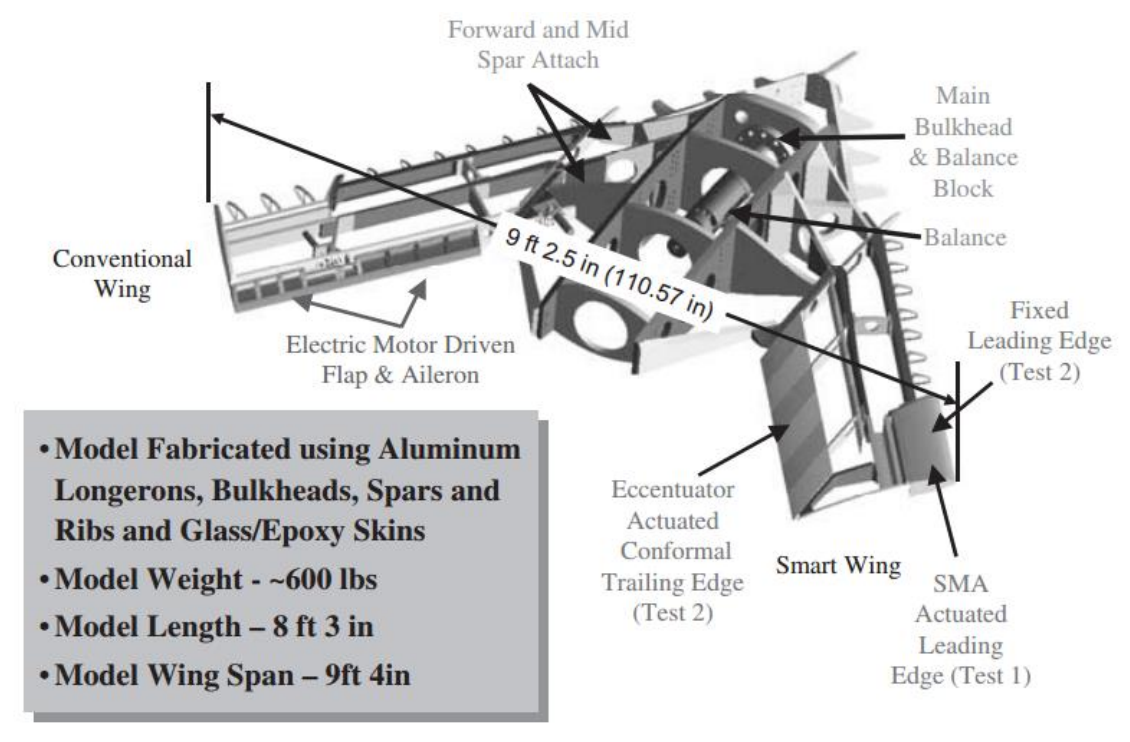

Figure 1.4 Smart wing model integrated the 'Eccentuator' and SMA actuators [12]

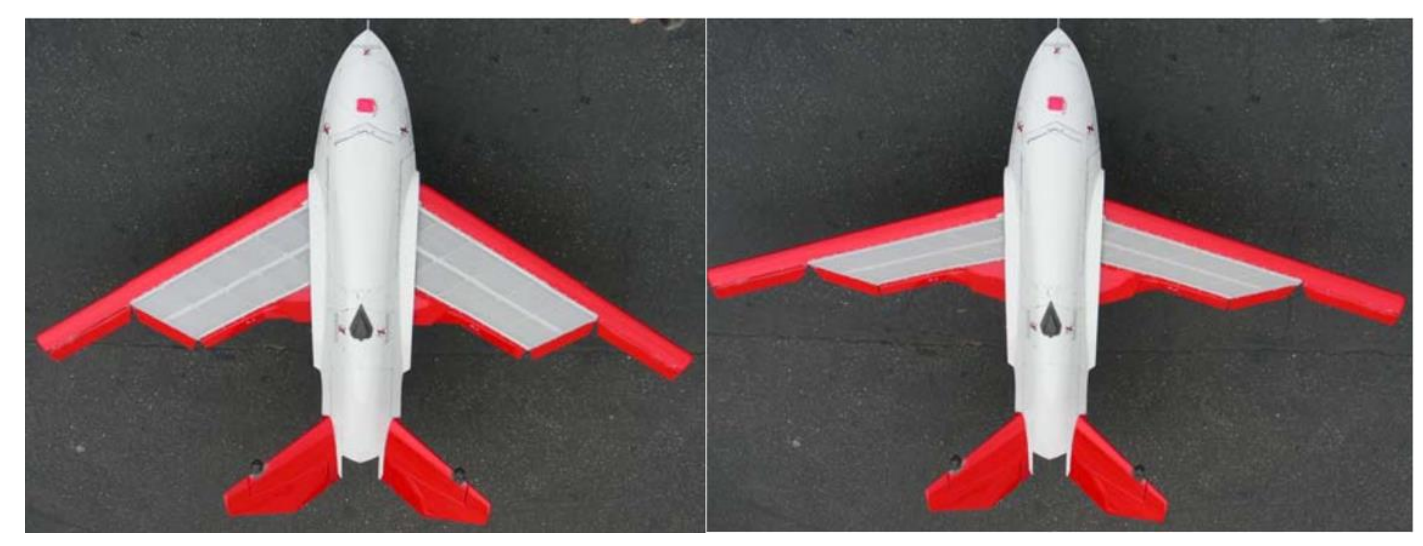

Figure 1.5 Change of the MFX-1 wing platform geometry [13]

\subsubsection{Challenges and opportunities in the development of morphing aircraft}

\subsubsection{Balance between shape-changing, carrying loads and weight}

In this section, some issues concerning the status of morphing aircraft are discussed.

During the past few years, many morphing concepts have been proposed and studied. The majority of the challenges concerns the structural design from the viewpoint of the system level. 
One of the difficulties remaining in the structural design of morphing aircraft is the sensitive balance between the flexibility to change the shape and the stiffness to carry the aerodynamic loads on the structure. According to Thill et al. [17], two basic approaches can be used to solve the dilemma, i.e. stiffness tailoring and actively controlled stiffness. One representative application of stiffness tailoring is the Fish Bone Active Camber (FishBAC) concept. The FishBAC was built using a highly anisotropic compliant structure, which had a very low chordwise bending stiffness but a high spanwise bending stiffness $[18,19]$. A fluid-structure interaction analysis was performed since the deflections of the morphing structures were large [20]. Alternatively, variable stiffness may be used, which usually relies on novel smart materials, such as shape memory polymers [21], or changes of the fluid pressure in the flexible matrix composite actuator [22].

The stiffness balance is more difficult if the requirements include the structural weight. A requirement triangle considering the three aspects was given by Campanile [23] and shown in Figure 1.6.

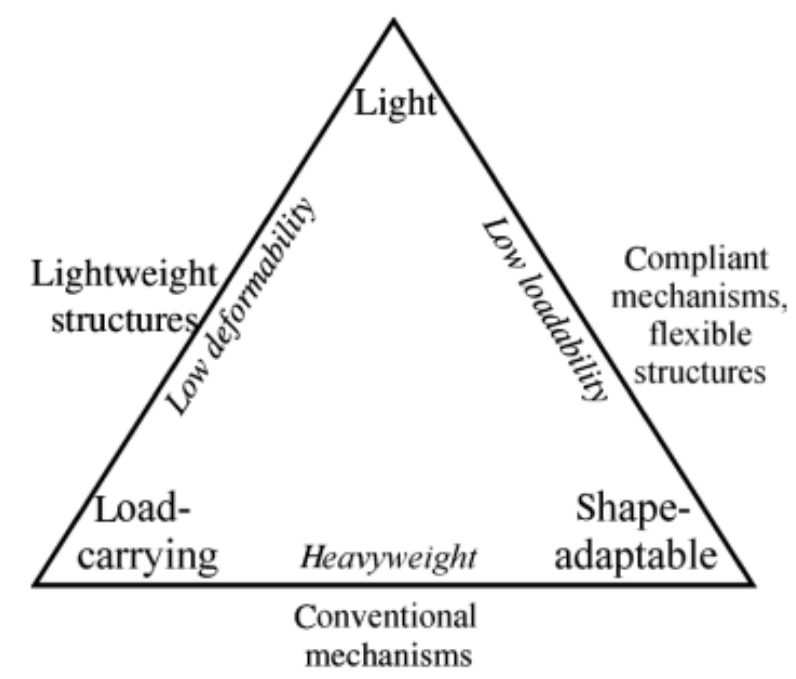

Figure 1.6 Requirements triangle illustrating the conflicts in morphing aircraft [23]

It may be easy to satisfy two of the three requirements simultaneously, e.g. the capability of geometry change and being lightweight. For instance, some deployable structures used in satellites can change their shapes and are very light. The capability of carrying loads and being light is the key objective of the optimisation of 
conventional fixed-geometry wing structures, which makes the structural weight the primary target.

However, it is challenging to meet all of the requirements. The requirements need to be satisfied simultaneously, but the problem can be solved in sequence. For instance, one approach is to minimise the weight of the structure, while taking the requirements of carrying the loads and shape-changing as the rigid constraints that have to be met.

On the system level, although adaptively changing the shape of the wing could bring potential benefits to the aircraft, the weight significantly influences the overall performance. Including shape-changing capabilities would inevitably lead to an increase in the weight. The final decision of whether the morphing technology is adopted must consider the performance to conduct flight missions, although the drive in the aircraft industry is to reduce weight. One cannot exclude the existence of an aircraft that is heavier, but capable of satisfying different requirements, with potentially improved performance, through the use of morphing technologies. It is necessary to consider both the positive effects of morphing technologies and the consequential weight change at the system level, and then to make a decision.

The weight information of aircraft can be obtained by a statistical analysis of existing aircraft or by sizing the structure using numerical simulations, such as the Finite Element Method (FEM). Due to the lack of historical data, the weight estimation of morphing aircraft can only be done by numerical simulation, which is also essential in the development of new morphing concepts. Compared to the development of new morphing concepts, the weight evaluation of morphing structures is quite limited. Skillen and Crossley reported morphing aircraft sizing techniques and a weight predictor based on the 'morphing wing template' [24-27]. Some typical morphing concepts, e.g. the folding wing and the variable sweep wing, were investigated, which enabled the fast modelling, analysis and evaluation of the morphing aircraft. However, the analysis is not comprehensive for the various morphing concepts due to the limited range of templates. 


\subsubsection{Compliant structure}

Compliant structures are structures that are flexible enough to induce the shape change using their structural deformation, rather than using mechanisms, such as hinges. In the field of morphing aircraft, the compliant structure is a fundamental concept. The development of the optimisation makes it possible for the compliant structure to change shape and carry aerodynamic loads simultaneously. Moreover, the rapid development of manufacturing technologies, such as 3D printing, makes some optimised structures, which are difficult to fabricate, become viable. The advantage of using compliant structures is the weight reduction compared to conventional mechanisms due to fewer mechanical components such as bearings. Also, avoiding using mechanical components could reduce the complexity of the morphing structure.

Many compliant structures have been proposed. Among the numerous designs, flexible corrugated panels and honeycomb structures are two fundamental types of structure. Both concepts have conventional origins, which have been widely investigated and applied in the academia and industry.

Corrugated panels are made of plates with periodic profiles, which can be used as cores in sandwich structures. They are widely used, such as in the packaging industry and in civil engineering. Although the finite element method can be used to evaluate the stiffness of the corrugated structures, the detailed modelling of the profiles would lead to a high computational cost, which motivates the study of equivalent models [28-30].

In recent years, the study of morphing aircraft motivated further research into corrugated structures [31-35]. The low axial stiffness of the corrugated structure allows for a large deformation with limited actuation force, while the high anisotropy of the corrugated structure provides a high out-of-plane stiffness to carry aerodynamic loads. A recent review paper summarised the applications of corrugated structures in morphing aircraft [36], in which the mechanics of the structures and morphing application examples are well classified and discussed. 
The honeycomb structure is another periodic structure, and conventional honeycomb structures have been widely used in the aerospace industry since they have a high specific strength in sandwich structures. The mechanics of conventional honeycomb structures was well summarised in [37]. Flexible honeycomb structures have been used in the design of morphing skins, for example [38-41]. The periodicity amplifies the small deformation of each unit, and the geometry creates a relatively high out-of-plane stiffness that is able to support material for the morphing skins.

\subsubsection{Actuation system}

An actuator is a primary component of morphing aircraft. The actuator provides the force or moment to change the shape of a structure but it increases the weight and complexity of the system. The conflict requires a high actuation force(moment) to weight ratio. The size of the actuator should also be able to fit in the space available in the morphing aircraft. Since the actuator is usually applied with associated mechanisms such as hinges and gearboxes, the constraints become more challenging and need to be solved by considering the entire system.

The selection of the actuator depends on the individual design. Some frequent options include conventional servo motors and novel smart materials such as piezoelectric material and shape memory alloys. Although novel materials or structures have been proposed and investigated at the beginning of the development of morphing aircraft, conventional actuators still have advantages with their better reliability, availability and lower cost.

A piezoelectric composite actuator known as the Macro-Fiber-Composite (MFC) was used to change the camber of a wing [42-44]. The piezoelectric actuator was flexible enough to be attached to the surface of the airfoil and could apply a relatively large strain to the structure. One disadvantage was the high voltage required to drive the actuator, which required suitable amplifiers. Another aspect required care was the method to bond the MFC actuator to the structure surface. Obviously, a secure connection between the actuator and the structure would ensure the deformation of the MFC could be transferred to the structure, while the increased 
stiffness caused by the adhesive could reduce the final shape change. The fatigue of the MFC actuator, as well as the bonding surface, was another challenge in its application, which demands more attention in future research. As discussed in Section 1.1.1, the slow response of the SMA actuator makes it challenging to achieve a high bandwidth. Also, the heating and cooling process would demand additional systems, which makes it even more difficult, especially if the morphing structure is far away from the power supply. An excellent example of the SMA actuator is the Variable Geometry Chevron (VGC), which was used to reduce the jet noise. The VGC makes use of the temperature variation from the take-off to the cruise phase to transform the SMA from the Austenitic form to the more flexible Martensitic form, which would be returned to the retracted position by the substructure [45]. This operation mode does not require external heaters since the temperature change was caused by the jet flow, and thus would be very promising if other aspects of this concept can be validated. However, it is not easy to take advantage of the temperature changes that exist in aircraft, and the active control of the temperature would increase the system complexity, reducing the benefits of morphing aircraft. In the Smart Wing project, ultrasonic piezoelectric motors were finally selected due to their high rated torque with a similar size and acceptable weight [46]. Except for the Smart Wing project, very little research could be found in the literature on the application of ultrasonic motors to change the wing shape [47], which might be explained by the limited maturity and availability. The FishBAC camber morphing concept employed the commercial electrical servo motors, which applied the moment to the structure.

If the focus of the research is on the structure not on the actuator, it is straightforward to use conventional electrical motors at the stage of laboratory development, considering its reliability, availability and cost.

Another essential feature of the actuation system is the self-locking capability if the actuator is expected to carry the aerodynamic loads. Due to its flexibility, the morphing structure would tend to have a significant deformation under the 
aerodynamic loads, and the actuator might have to carry part of these loads. The self-locking capability could help to reduce the energy consumed by the actuator and ensure the shape of the aircraft is as required. The self-locking capability could be achieved mechanically by using worm gears and racks but these mechanisms would often demand a substantial weight increase.

\subsection{Review of morphing wingtip devices}

\subsubsection{Brief introduction to fixed-geometry wingtip devices}

The development of wing tip devices can be traced to Lanchester's vertical endplates in the late 1890s. At that time the endplate was called a 'capping plane' and proposed to 'minimise the loss of energy' of the flow [48]. However, the early-stage wing tip endplates were only useful at high lift coefficients. During cruise conditions, the profile drag caused by the endplates would compromise the benefits of the reduced induced drag [49]. Whitcomb proposed the winglet, which was a wing-like surface at the wing tip [50]. The winglet is a small wing, which has an aerodynamic cross-section and produces lift and drag. Installing the winglet can reduce the induced drag and weaken the wingtip vortices. Although increasing the wing span can also reduce the induced drag, installing the winglet rather than simply extending wing tip will have a smaller weight penalty. According to the literature [49], for the same wing root bending moment, the winglet can halve the induced drag compared to a pure wingtip extension. Also, installing the winglet is better able to meet the constraints of airport dimensions.

Whitcomb's proposed solution shows that a thoughtful aerodynamic analysis is demanded for an advantageous design of the wingtip devices. Aerodynamic optimisation using (high fidelity) numerical calculation is often applied to design the winglet, such as [51-53], as well as requiring experimental validation, such as [54].

The optimisation of fixed-geometry wingtip devices is usually performed for a single design condition, which leaves enough scope for morphing wingtip devices. The 
KC-135 was evaluated with different wingtip devices: winglets, raked wingtips and wing span extension [55]. Different geometries were tested to find an optimum design although the range of the geometry parameters was limited. The results were used to provide a criterion to determine whether it was beneficial to retrofit the $\mathrm{KC}-135$ and what modifications should be made to the wingtips, but the process to evaluate different geometries also indicated the potential of applying morphing wingtip devices, which can change the wingtip shape adaptively. The modelling and analysis of fixed-geometry wingtip devices at different flight conditions were conducted using a databased prediction tool [56]. The results showed that the drag reduction at the high-speed condition could be linked to the drag penalty at the high lift condition, and the authors suggested one option to overcome the conflict could be the application of moving parts at the leading and trailing edge of the wingtip devices.

\subsubsection{Research on morphing wingtip devices}

\subsubsection{Background and classification}

A fixed winglet can only be optimised to meet the requirements of a specific flight condition. As a branch of morphing aircraft, the research on morphing wingtip devices follows the same philosophy: changing the shape adaptively to meet different requirements.

The potential benefits of morphing technologies in the design of wingtip devices have aroused the attention of many researchers. Similar to morphing aircraft, there are several ways to classify morphing wingtip devices. Figure 1.7 shows a partial summary of the research activities and classifies them from different perspectives, i.e. by the motivation, the application scenario, or the technology applied. 


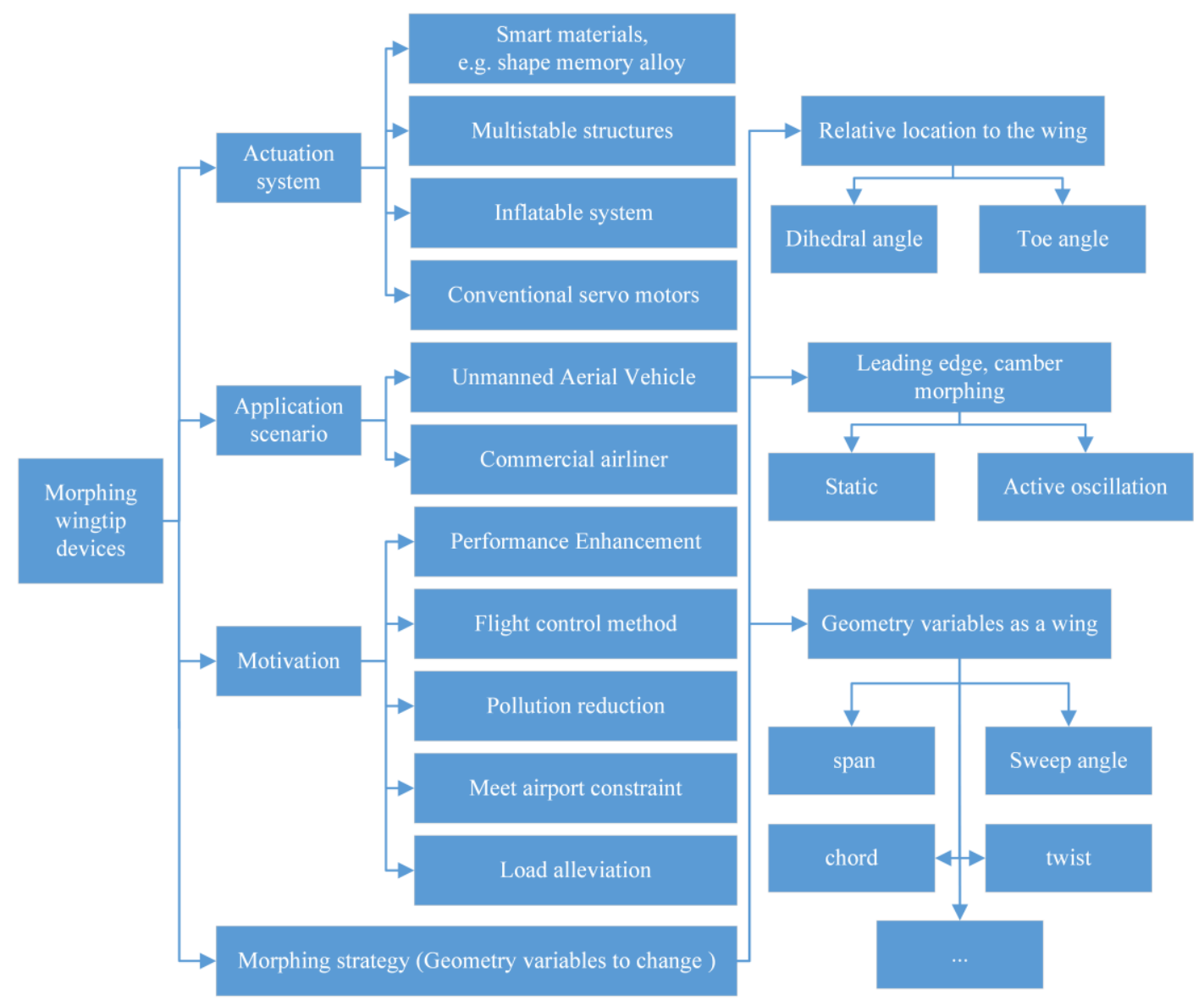

Figure 1.7 Classification of morphing wingtip devices

The classification of morphing wingtip devices has more than one approach. Moreover, reviewing the literature suggests the research on this topic is usually comprehensive, and crosses different disciplines. Since the winglet is wing-like, the morphing concepts for other morphing wing designs could also be applied to the morphing wingtip devices.

The morphing variable, corresponding to the geometry parameter, which is changed by morphing aircraft, can be used to differentiate the morphing concepts. If the morphing variable has been extensively investigated by other morphing concepts, and a similar technology approach can be applied to wingtip devices, then only a brief introduction to them is given; this includes camber morphing, wing span morphing, the change of sweep angle or wing area, etc. For example, Koreanschi et al. $[57,58]$ applied airfoil morphing at the wingtip region close to the winglet. The upper thickness of the airfoil was changed by four actuators and was optimised with 
their in-house genetic algorithm to minimise the drag. A morphing wingtip demonstrator was built and tested in the wind tunnel. Vasista et al. [59] applied the droop-nose leading edge morphing at the wingtip. A complete design chain was used for the morphing wingtip, including the three-dimensional optimisation of the skin, topology optimisation of the compliant mechanism for the actuation, manufacturing and assembly. As shown in Figure 1.8, experiments were performed to assess the morphing structure under aerodynamic loads. Lessons were learned on several aspects of the morphing wingtip [60], which gave meaningful experience and recommendations to morphing structures on a larger scale.
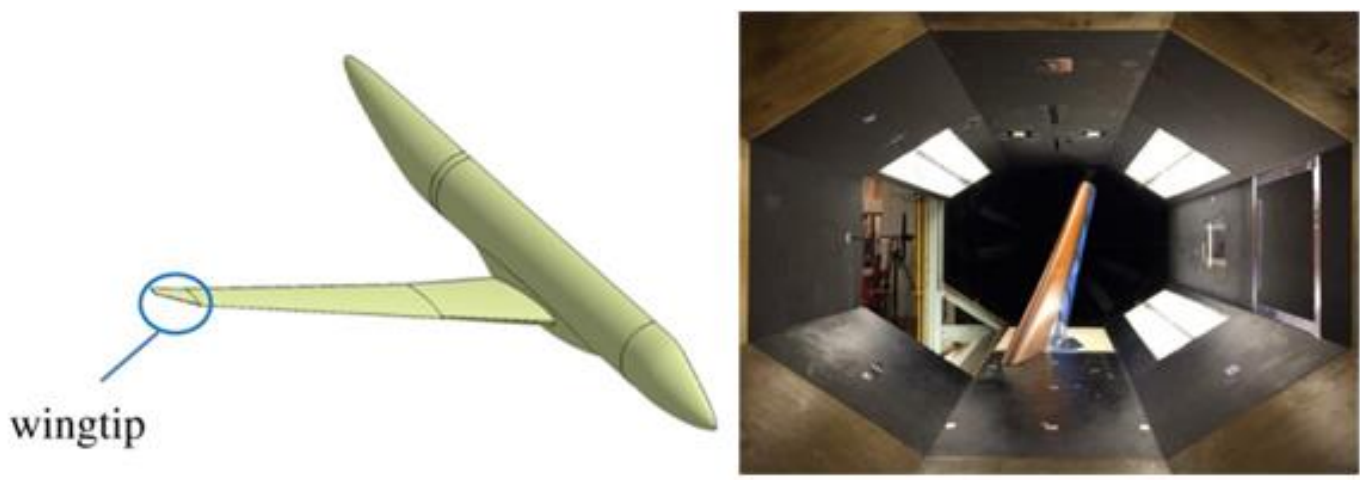

Figure 1.8 Droop-nose morphing leading edge at the wingtip: (a) Concept, (b) Wind tunnel model [59]

A well-known commercial example is the 'Active winglet' developed by Tamarack ${ }^{\circledR}[61]$, which has been certified and applied on business jets. As shown in Figure 1.9, the active winglet consists of two parts: a wing span extension with a control surface, and a traditional winglet. The traditional winglet is able to increase the lift to drag ratio, resulting in a better flight performance as other winglets do. The control surface is retrofitted and can provide load alleviation capabilities by its deflections. By integrating the control surface into the wingtip, the winglet becomes active and can yield 3 to 4 times fuel saving compared to traditional winglets.

More attention is focused on the morphing variables that only apply to the wingtip devices, such as the dihedral angle and the toe angle of the winglet. Another focus will be the morphing concepts that are merely developed for the wingtip devices, which includes multistable wingtip structures, inflatable wingtip devices, flight 
control using morphing wingtips, and morphing wingtips for load alleviation. Active flow control using morphing wingtip devices is also briefly reviewed.

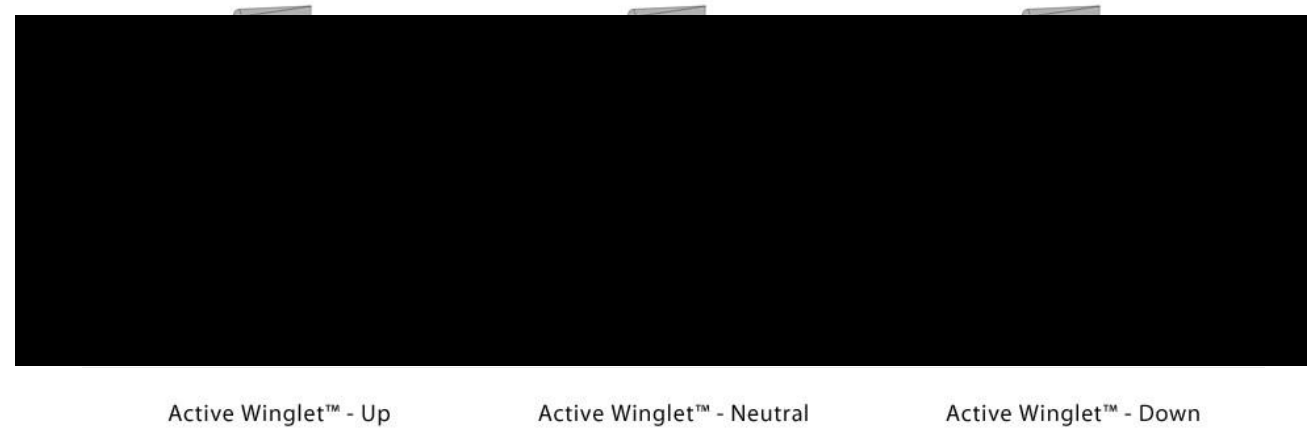

Figure 1.9 Active winglet explanation (http://www.rapp.org/archives/2012/10/aopa-summit/)

\subsubsection{Morphing wingtips devices that change its relative location}

If the winglet is taken as a single component, the change of its relative location to the wing becomes the source of the morphing variables. The cant angle is the angle of the winglet with respect to the vertical axis. The dihedral angle can also be used to describe the position since the two angles are complementary angles. Another geometry parameter frequently seen in the literature is the twist angle of the winglet. Changing the cant angle will induce a direct and substantial influence on the aircraft, and has become a priority direction in the field of morphing wingtip devices. The change of twist angle can influence the local angle of attack of the winglet, which also affects the global aerodynamic performance.

A typical example of changing the dihedral angle is the folding wing used to save space in aircraft carriers. The on-going Boeing $777 \mathrm{x}$ airliner has a folding raked wingtip to reduce the span on the ground [62] as shown in Figure 1.10. While the newly designed wing of $777 x$ provides excellent flight performance, the large wing span makes it unsuitable to meet airport dimension constraints. During landing, the wingtip can be folded automatically if the airliner is below a set speed. For take-off, the wingtip can be deployed manually as part of the pre-takeoff checklist [63]. By folding and unfolding the wingtip, the flight performance can be improved while the 
constraint of the airport is still satisfied. Another folding wing example was the XB-70 Valkyrie as shown in Figure 1.10(b), which used the folding wingtip to improve the flight performance during its supersonic flight.

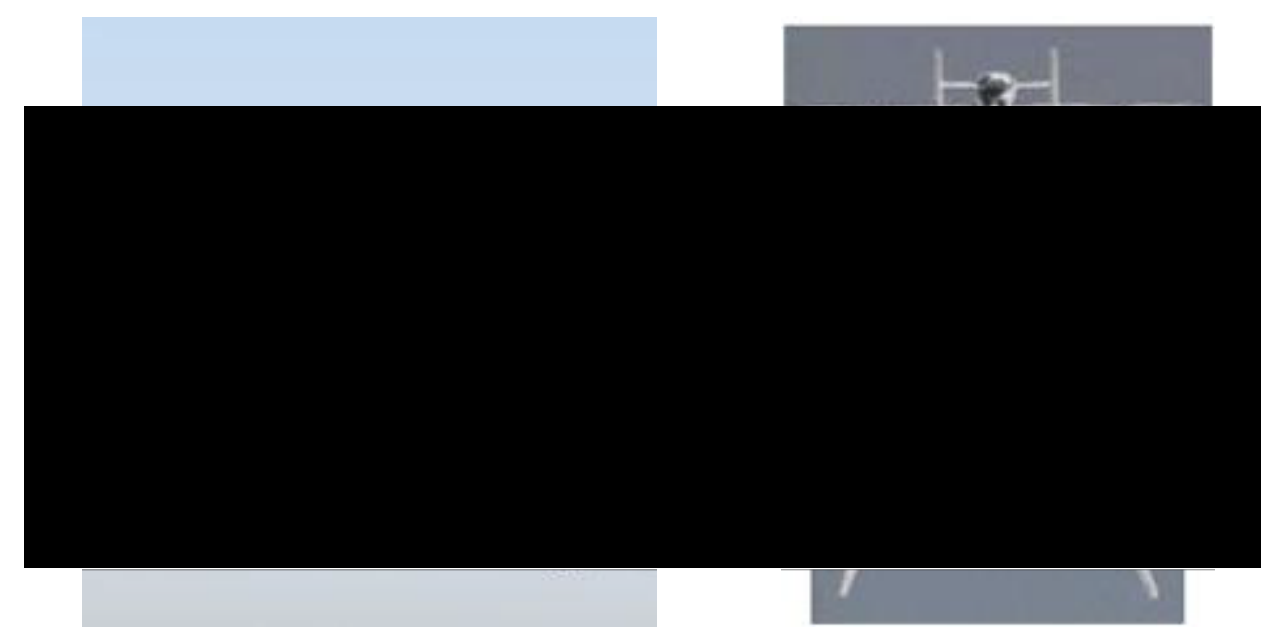

Figure 1.10 (a) Boeing 777x folding wingtip

(http://www.boeing.com/commercial/777x/by-design/\#/featured), (b) XB-70 wingtip devices at different angles (http://xb70.interceptor.com/)

Although the folding wing is not strictly a morphing structure since it only works on the ground or the deck of an aircraft carrier, it gives some insights into the research on morphing wingtip devices. The 'Spanwise Adaptive Wing (SAW)' project was launched recently by NASA [64]. As shown in Figure 1.11, a significant rotation of the outboard wing is expected. Not many details are currently available but it can still be confirmed that an SMA based rotary actuator will be used by considering its weight and size advantages compared to a conventional hydraulic system. Flight tests show that the SAW can provide $40 \%$ of total rudder authority, which might be used to reduce the rudder size for better flight efficiency.

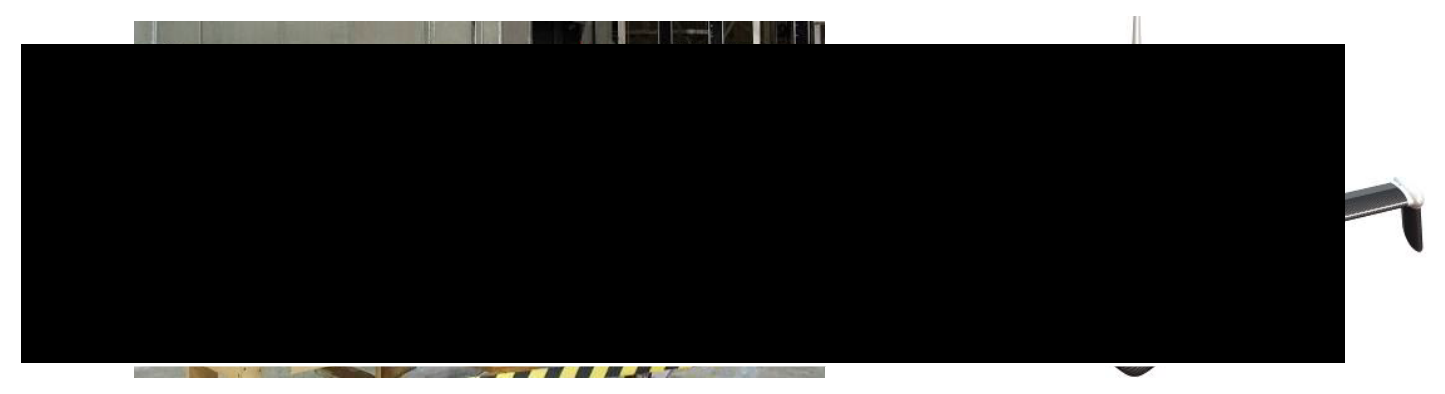

Figure 1.11 SAW: (a) Un-morphed, (b) Morphed (imaginary) 
(https://www.nasa.gov/feature/nasa-to-test-in-flight-folding-spanwise-adaptive-wing -to-enhance-aircraft-efficiency)

In the literature, three main motivations can be found to change the relative location of the winglets, which are also potential applications of the morphing wingtip devices.

\section{1) Performance enhancement}

The MORPHLET (MORPHing wingLET) was proposed by Ursache et al. [65]. The Nonplanar configuration of the morphing winglet could change the dihedral angle, twist angle, span and taper. The morphing winglet was optimised to improve the specific air range of a narrow-body airliner for various flight conditions. A decision-making process was explained to select a smart actuator for the morphing winglet and the SMA actuator was selected. The optimisation included a low-fidelity aerodynamic analysis module and a weight prediction module, which revealed candidate configurations for the corresponding flight phases. A demonstrator was manufactured to validate the technologies integrated on the morphing winglet [66]. The demonstrator was only supposed to provide the morphing shape but not to carry aerodynamic loads. The material properties of the MORPHLET skin were investigated numerically and experimentally as shown in Figure 1.12(a). A Kevlar corrugated skin was used and a manual drape was employed to achieve the complex tow drape pattern. Although the manual drape with large ply size caused a defective lay-up at the leading edge, the process still provided meaningful lessons that can be learned by other researchers. For example, the numerical simulation indicated the buckling of the skin, which should be avoided by reinforcement of the corrugated skin.

The work was expanded to a multidisciplinary design optimisation (MDO) including the structure model, weight estimation, engine model and performance analysis, in which the necessary aircraft design parameters could be sized [67]. Then, the MDO suite was used to analyse multiple flight phases, which gave a $6 \%$ specific air flight improvement compared to the baseline aircraft, as well as a $4.5 \%-5.5 \%$ increase for 
all analysed phases. The MDO suite was even improved by incorporating a multi-objective function, which could combine the objectives for each flight phase [68]. The separate optimisations for each phase were transformed into a single optimisation, in which the objectives of each phase were optimised simultaneously. A structural module, called UC700, was developed to size the structure and estimate the weight [69]. Some low-speed operation performance parameters were also analysed to make a comprehensive analysis of the potential benefits of the morphing winglets. The results showed a significant (4-6.6\%) increase in the specific air range across all flight phases, and a $3.1 \%$ lift to drag ratio increase in the climbing phase, which was higher than the Sharklet wingtip retrofitted to the A320neo. The optimal cant changes during different conditions are shown in Figure 1.12(b). High fidelity computation and wind tunnel tests were performed to validate the morphing winglet concept [70].

A morphing wingtip device based on a chiral structure has been proposed [71]. Agent models based on a neural network were built to conduct a sensitivity analysis of the aerodynamics and wing bending moment. The dihedral and twist angle were found to have more influence on the wing root bending moment, as well as the wingtip bending moment. A $2 \%$ fuel saving could be obtained if the mission range was a 1000-km journey with maximum payload. The research pointed out that changing dihedral, twist angles and camber of the morphing wingtip was challenging due to the limitation of the actuators. An initial study of passive gust load alleviation also indicated a 5-12\% reduction of the wing root bending moment could be achieved, and more literature in the field of load alleviation using wingtip devices will be introduced in the next section. 


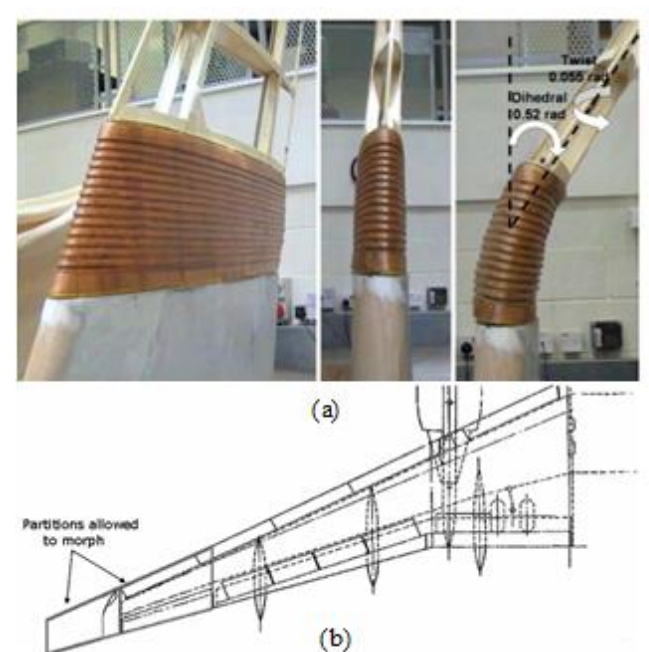

(b)

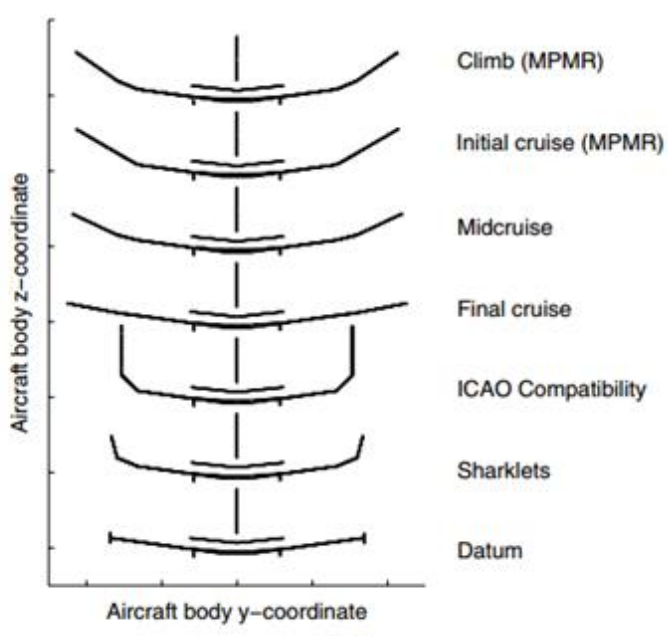

(c)

Figure 1.12 (a) A demonstrator using corrugated skin [66], (b) The partitions of the winglet retrofitted to the fixed wing [68], (c) Optimum dihedral angles of the morphing winglet [68]

Falcao et al. [72] optimised a morphing winglet, which could change its dihedral and toe angles to reach a performance improvement of an unmanned aerial vehicle. A hinged mechanism was designed and tested to change the two angles independently. Aero-structure interaction analysis was implemented to size the morphing structure while considering aerodynamic loads. A significant increase of the take-off roll moment was reported, and the mechanism has been validated preliminarily using a prototype. The mechanism was further investigated and tested in [73]. Two commercially available servo motors were used to rotate the winglet about two axes, which corresponded to the change of the dihedral and toe angles respectively. A dynamic test was also performed to prevent any harmful dynamic characteristics that occurred from replacing the fixed winglet with the morphing winglet. However, the hinged mechanism was not covered by any skin in the reference, which would cause additional drag in the real application, and might even offset the benefits of the morphing winglet.

\section{2) For flight control}

The optimal results in the MORPHLET project showed a significant change of the winglet cant angle to meet the different flight conditions, although it was not the first 
application of a variable cant winglet. The variable cant angle winglet was initially proposed by Bourdin et al. [74, 75]. As shown in Figure 1.13(a), the idea was using independent actuated winglets to achieve basic manoeuvers of a flying wing model. Wind tunnel tests, together with numerical calculation, suggested adequate roll and pitch moment could be obtained from this concept. The drawback was the highly coupled control system since the winglets could generate moments along multiple axes. With this coupling, a banked turn of the flying wing could only occur with a specific radius if the winglets were the sole control effector.

A refinement was conducted to expand the flight envelope with a second pair of morphing winglets [76]. The two pairs of winglets split the wingtip and allowed independent changes of the winglet dihedral angle. With four control effectors, the flying wing was over-actuated. More than one set of winglet dihedral angles could be used to achieve the same manoeuver, which might provide additional morphing benefits, such as performance enhancement and load alleviation.

The surface pressure of the flying wing was also measured, which concluded that the dihedral angle of the winglet had a substantial influence on the main wing surface pressure [77].
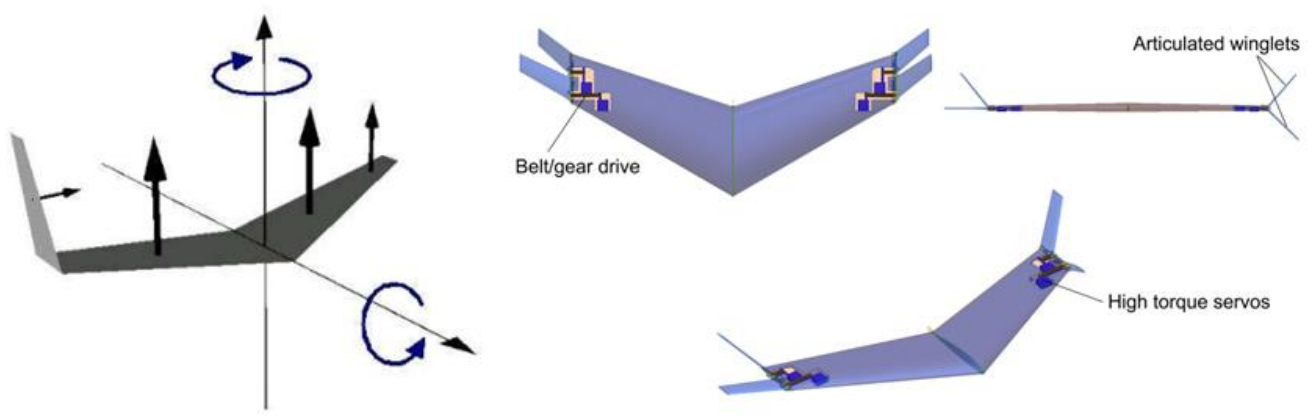

Figure 1.13 (a) Unsymmetrical winglet arrangements [74], (b) Two pairs of winglets retrofitted to provide additional control capability [76]

3) For Load alleviation

Load alleviation using a folding wingtip, which inherently changed the dihedral 
angle of the winglet, has been investigated [78-81]. The idea was based on a flexible hinged wingtip, which would be passively actuated to fold when the aircraft encountered gust loads. It was found that the orientation of the hinge line, about which the wingtip rotates, was a critical parameter for successful load alleviation. If the hinge line was not parallel to the flow direction, folding the wingtip would change the local angle of attack. A nonlinear hinge was modelled and investigated [79]. The nonlinear hinge would only allow the wingtip to rotate if the hinge moment became larger than some predefined threshold value. It was highlighted that the threshold value could strongly affect the load alleviation capabilities. Furthermore, a negative stiffness nonlinear hinge was developed to improve the load alleviation capabilities [80]. As shown in Figure 1.14(b), wind tunnel tests were performed with a $30^{\circ}$ hinge angle for both steady flow tests and gust excitation tests. The gust excitation tests showed a stiff-hinge would have worse capabilities of load alleviation than the free-hinge and sprung-hinge. Generally, from the literature, it was possible to develop a passive hinge based wingtip for load alleviation, while the careful selection of the device parameters is required for successful designs.
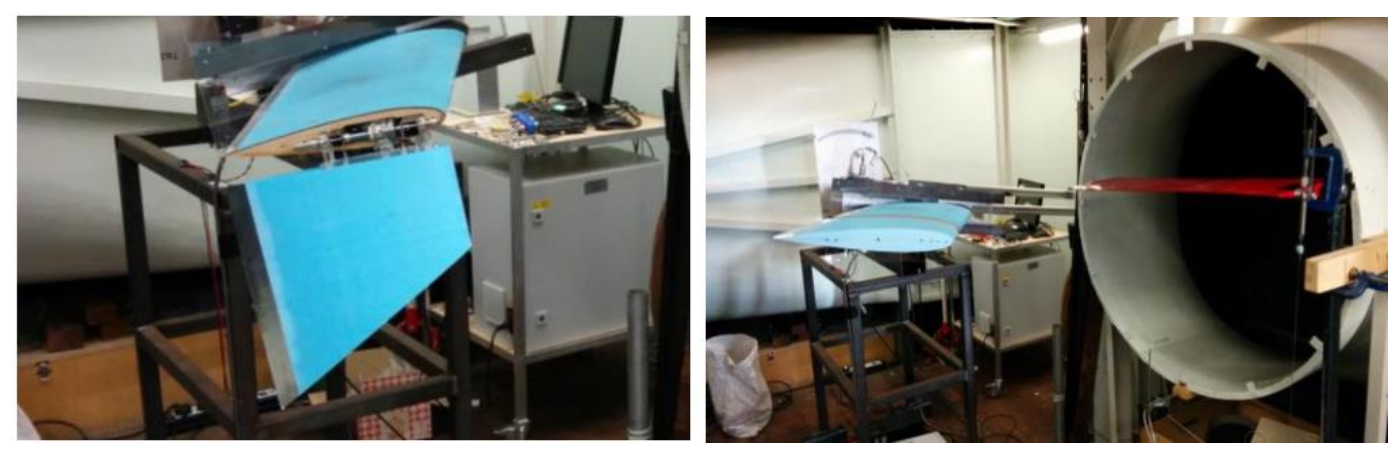

Figure 1.14. (a) Folding wingtip device, (b) Gust excitation test [81]

Gust load alleviation using a passive wingtip could also be achieved by allowing the wingtip to reduce the angle of incidence and the associated aerodynamic loads [82-86]. A wingtip device was proposed in [82-84]. The wingtip was connected to the inboard wing section by a torsional spring. The aerodynamic centre of the wingtip section was aft of the rotation axis (determined by the location of the spring), which would cause the nose-down rotation of the wingtip when the wing encountered an up-gust in the airflow. An approximate aeroelastic model was 
developed and applied to perform a parametric study [84] and in the case study for a sensor aircraft, mass and stress reductions were achieved. Wind tunnel tests were also performed to test the concept [83], in which the motion of the wing would be reduced when the wingtip device was applied. This wingtip device was named a 'discrete wingtip design' in [85] since the wingtip was essentially retrofitted to the baseline wing. The design was extended to the 'composite wingtip design', in which the wingtip was a span extension to the wing and the load alleviation was achieved through aeroelastic tailoring of the wingtip. A similar wingtip design was also proposed in [86] for a large aircraft.

The capability of load alleviation of the passive wingtip devices depended on the wingtip mass, hinge/torsional stiffness, hinge orientation/torsion axis location, etc. From the author's perspective, it could be worth using an active morphing wingtip since the actuation force would be able to provide more control of the final deformation of the wingtip, which primarily determines the loads on the wing.

\subsubsection{Morphing wingtips devices based on unconventional technologies}

In the previous subsection, the literature on the motivations for morphing wingtips was briefly reviewed. To achieve the shape change in the wingtip devices, some unique technology approaches are necessary due to the limitations of the size, space and actuation requirements, which are summarised below.

\section{1) Shape Memory Alloys (SMA) based actuation}

The limited size and available space at the wingtip lead to more challenging actuation systems than other morphing wing designs. A soft morphing winglet was proposed by Han et al. [87]. As shown in Figure 1.15(a), the SMA wires were embedded into a soft polymeric matrix together with the glass fibres to make a self-actuated winglet. The winglet could vary from the flat configuration to curved when it was actuated, as shown in Figure 1.15(b). A current was applied to activate the SMA wires, and the wire diameter was $300 \mu \mathrm{m}$. An SMA spring was used as the actuator to change the cant angle of a morphing winglet [88]. The drawbacks of 
using the SMA actuator are the low response speed and the requirement of external thermal activation, as clarified in Section 1.1.2.3.
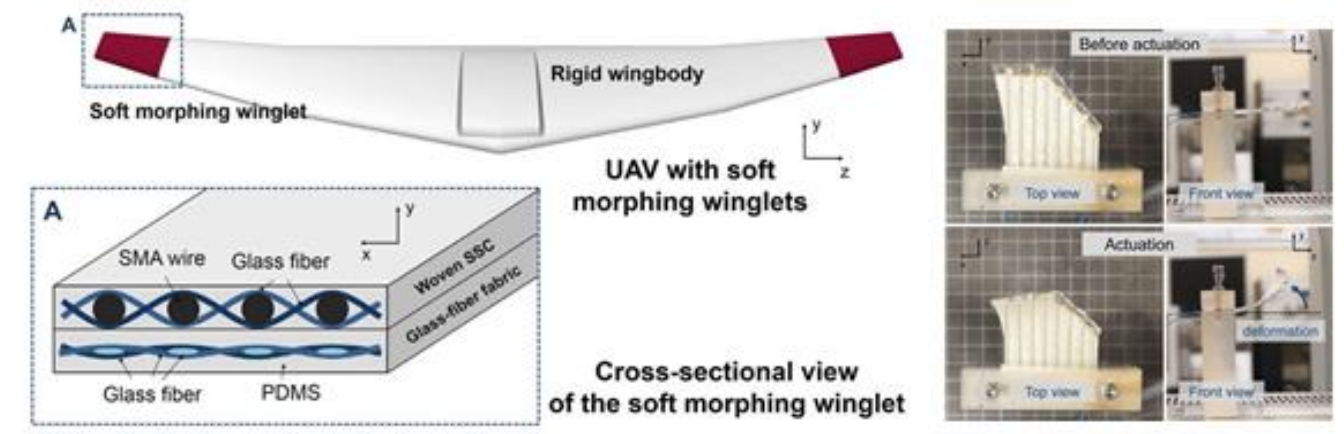

Figure 1.15 (a) Concept of the SMA based soft morphing winglet, (b) Actuation test

2) Inflatable system

An inflatable system might be able to maintain its shape under aerodynamic loads due to the small size of the wingtip and the relatively small loads in the region. An inflatable telescopic wingtip design was proposed by Daniele et al. [89]. As shown in Figure 1.16(a), an inflatable winglet was attached to the end of a fixed-geometry wing. The telescopic device was assumed to carry all the aerodynamic loads of the winglet, which were supported by a 'two-spar' design. Evaluation of the flight performance considering both the aerodynamic performance increase induced by the morphing wingtip and the weight penalty of the inflatable device was performed, which suggested a reduction in the direct operation cost as large as $400 \$ / \mathrm{h}$ for an aircraft similar to the A380. Moreover, a $2 \%$ reduction of the global warming potential could be obtained, which showed significant environmental benefits.

Another example of an inflatable system used pressurised tubes in auxetic honeycomb structures [90]. The auxetic honeycomb structure had a negative Poisson's ratio and expanded its volume under tensile loading. As shown in Figure 1.16(b), the pressurised tubes were in the honeycomb structures and could help to keep the wingtip horizontal with the keyway. If the pressure in the tubes were removed, the wingtip tended to deform the honeycomb structure and rotate about the 
hinge. The honeycomb would deform towards the wing box while avoiding the generation of a bump due to the auxetic effect. If the pressure in the tubes was increased, the wingtip would be actuated to return to the horizontal position and carry the aerodynamic loads. The inflatable system enabled the wingtip to change the dihedral angle, and preliminary results validated the concepts by analytical and numerical calculation and by experiments.

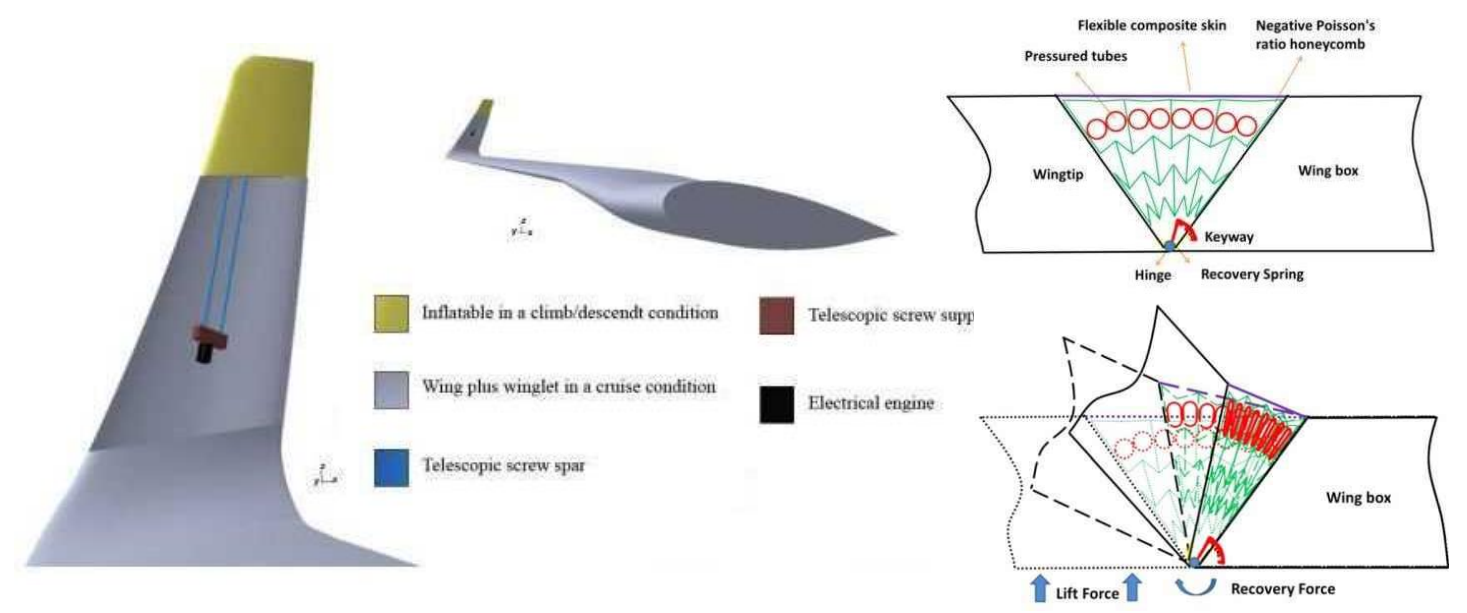

Figure 1.16 (a) Inflatable telescopic winglet [89], (b) auxetic honeycomb structure actuated by pressured tubes [90]

\section{3) Multistable structures}

Multistable structures have several stable states. They can carry loads until the external loads reach a certain point, where they then snap one equilibrium state to another. Using multistable structures can save actuation energy since the structures can carry certain loads. If only two stable states exist, the multistable structure becomes a bistable structure. Adopting the concept into morphing wingtip devices might suit the requirements of the actuation and size. Gatto et al. [91] investigated the potential of using a bistable composite winglet to enhance the take-off capability. The winglet was proposed to snap from its low-speed configuration (before take-off) to the high-speed configuration (for cruise/climb). The aerodynamic loads would activate the snap-through as the flight speed increased. Samples were designed and manufactured. Wind tunnel tests were undertaken to validate the concept. One side 
effect was the significant dynamic loads caused during the snap-through, which were transmitted to the entire wing structure. The dynamic loads might cause unexpected effects to the aircraft, and should be investigated to ensure no serious damage would occur. Passive load alleviation could be achieved by using the bistable morphing winglet [92]. As shown in Figure 1.17, when the external loads exceeded a certain point, the bistable winglet would jump from the high lift configuration to the load alleviation configuration, which generated less lift and alleviated the loads on the structure. To control the configuration change, MFC actuators were attached to winglet, and could dynamically induce the configuration change from one state to the other one, as well as reverse it [93]. The bistable phenomenon is not restricted to composites. Kim et al. proposed a metallic bistable structure to obtain a substantial dihedral angle change with a small size [94].

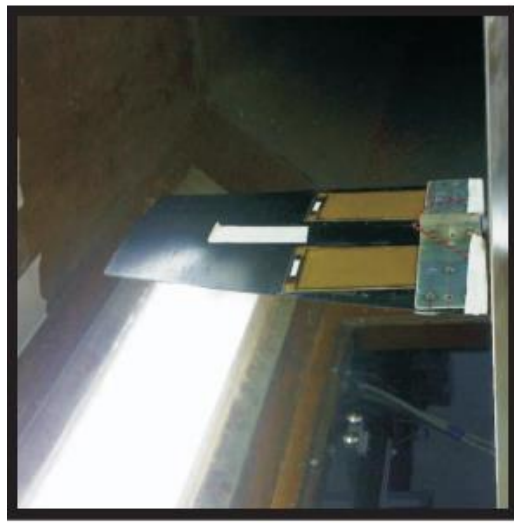

State 1

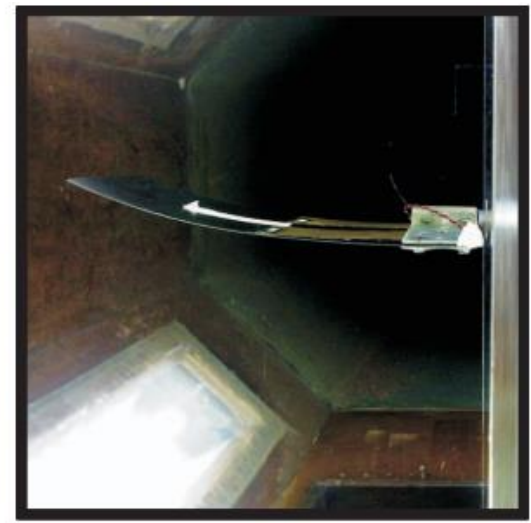

State 2

Figure 1.17 Bistable winglet (State 1: high lift configuration, State 2: load alleviation configuration) [92]

4) Bio-inspired multiple winglets

To mimic the tip feathers of some birds, active multiple winglets in an unmanned aerial vehicle were proposed, modelled and analysed [95]. The multiple winglets are shown in Figure 1.18. Numerical simulation found a significant (40\%) increase of the endurance and range compared to the baseline design with the same aspect ratio. It also indicated the possibilities of achieving load alleviation and replacing control surfaces. Wind tunnel tests were performed to analyse the aerodynamic 
characteristics of a half-wing model with multiple winglets [96]. Configurations with different winglet cant angle arrangements were tested and compared. The results indicated it was possible to find an optimal configuration although the comparisons were limited and more tests are required.
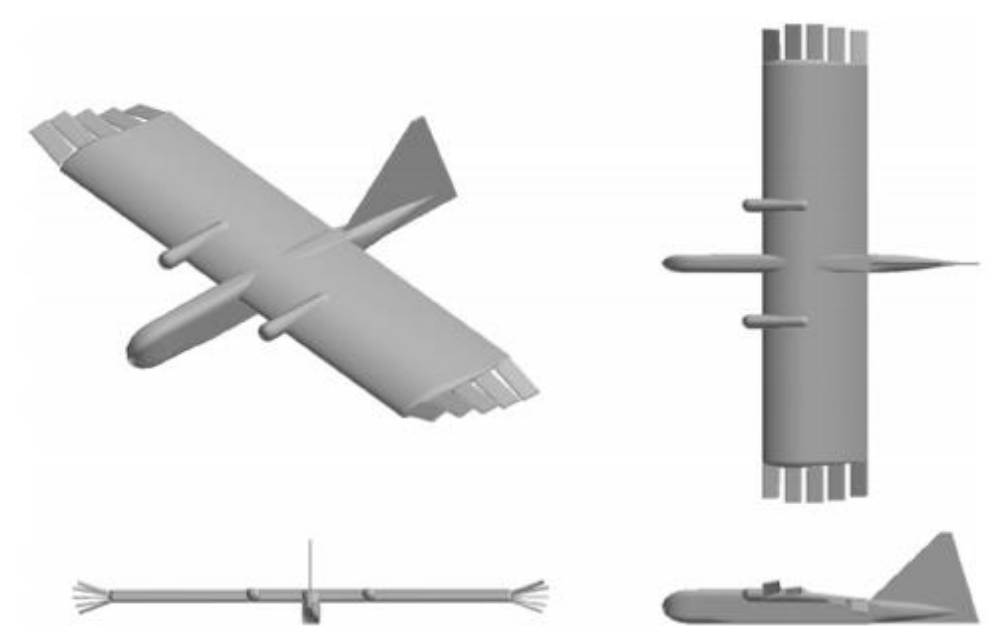

Figure 1.18 Multiple winglets on an unmanned aerial vehicle to mimic tip feathers of birds [95]

\subsubsection{Active flow control using morphing wingtip devices}

Although active flow control might have a longer history than morphing aircraft, their objectives are similar as morphing also intends to change the flow using the aircraft shape change. In this section, some morphing wingtip devices are listed since their motivation is to control the wingtip vortices.

Strong wake vortices are hazardous to the following aircraft, and oscillating control surface can accelerate their decay. Research on using the control surfaces on the winglets have been found in [97, 98]. As shown in Figure 1.19(a), control surfaces were added to the winglet's trailing edge, and test results have indicated that oscillating the control surfaces with a frequency matching the frequency range of the wake instabilities could significantly affect the wake vortices, at least in the near field [98]. 

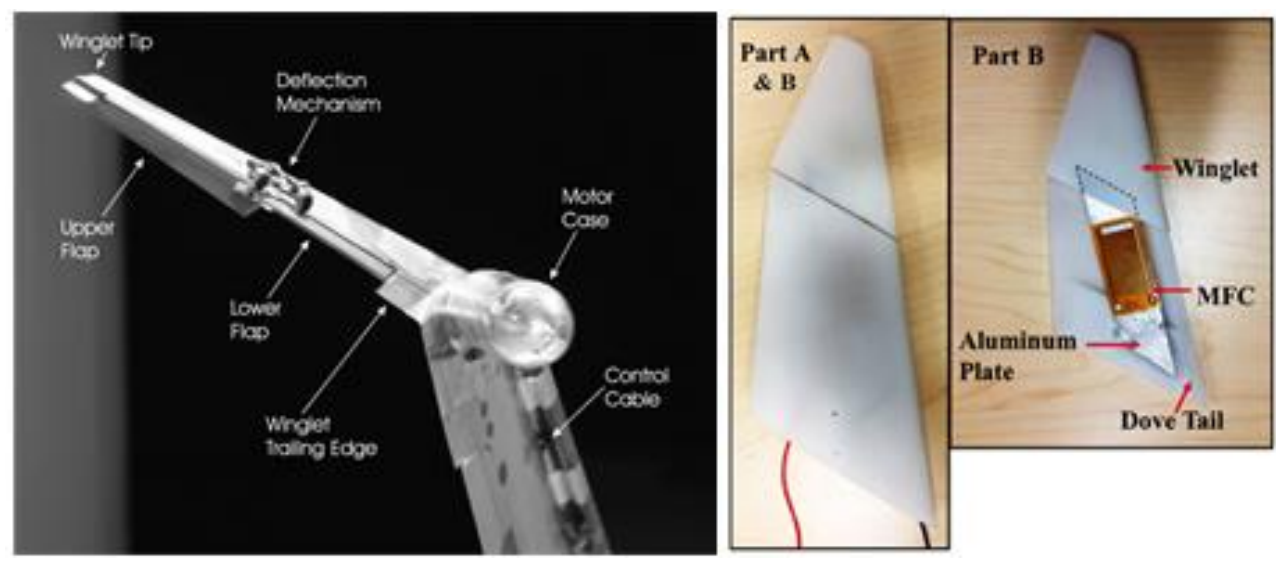

Figure 1.19 (a) Control surfaces on the winglet for alleviating wake vortices [98], (b) MFC actuators to oscillate the winglet [99]

Although conventional control surfaces were used in the study, it did not exclude the possibility of using a morphing camber, which is still an open question. Oscillations of the winglets could also be achieved by using piezoelectric materials. Experimental studies were performed by Guha et al. [99, 100]. As shown in Figure 1.19(b), MFC actuators were attached to the winglet model to oscillate the winglets. The MFC winglet had bimodal characteristics, and had negligible structure vibrations at its natural frequency if not actuated; however at the highest excitation, the winglet tip could have an oscillation amplitude as much as almost four times the airfoil thickness. The mean peak vorticity was reduced by $10 \%$ at all downstream locations, and the mean total circulation was reduced by $2 \%$ when the actuators worked at the highest excitation level.

\subsubsection{Summary}

While there has been much progress to design morphing wingtip devices, some problems remain open to solve. For example, the method to heat and cool down SMA actuators needs further investigation, especially when the required response speed is high. The application of multistable structures could be limited since only discrete shape changes can be obtained. The conventional servo motor is a reliable actuation option, but a morphing skin is still needed to provide a continuous aerodynamic surface for the morphing winglet. 
Another feature is the fast growth in the field of morphing wingtip devices. Over $66 \%$ of the literature reviewed in this Section 1.2.2 has been published in the past five years, and $46 \%$ in the last three years. The fast increase in the number of publications indicates the vitality of the topic, and also suggests more research is necessary to solve the remaining problems, which is one of the primary motivations of the thesis.

\subsection{Motivations of the research}

The primary motivation of the thesis is to try to answer the two fundamental problems in morphing aircraft research: whether it is worthwhile employing morphing technologies and if worthwhile, how to implement the proposed morphing concepts.

The two problems describe the main research work in the field of morphing aircraft. The potential benefits that morphing aircraft can bring need to be evaluated considering the advantages and drawbacks simultaneously, which inherently requires a system level study. Also, comparisons to conventional aircrafts are necessary if the morphing concept is a replacement or improvement to conventional designs. A baseline design should be modelled to highlight the differences.

In the thesis, a baseline aircraft will be retrofitted with the morphing wingtip devices. By investigating the morphing wingtip devices, the two fundamental questions can be answered in this particular case. There exist several reasons to take the morphing wingtip devices as an example:

1) Research on the morphing wingtip devices has not reached its limit.

Although there has been some literature on morphing wingtip devices as summarised in Section 1.2.2, the relevant research work is not comprehensive even compared to the work on other morphing concepts. More work is still necessary to investigate the growing morphing technologies applied to wingtip devices, particularly regarding the small size of the wingtip. 
2) Relatively large aerodynamic effects can be introduced despite the small size of the wingtip devices.

The size of the winglet, or other wingtip devices, is small compared to the size of the wing, although the fixed-geometry winglet has shown a good performance increase. Enabling the winglet to change its shape adaptively could significantly increase the performance level. The long distance between the wing root and the wingtip also makes the load change introduced by the wingtip devices significant, which might provide an excellent approach to load alleviation.

3) The winglet is a suitable component to try innovative morphing concepts, which would not introduce too many changes to the other parts of the aircraft.

The winglet is not a primary structural component, and can be retrofitted to existing wing designs. This inherent feature makes the practical validation of the morphing winglet much easier compared to other morphing wing concepts. The changes to the remaining design can be minimized, which ensures the safety of the primary structure as well as speeding up the development process.

However, there do exist some drawbacks if wingtip devices are selected, such as

1) The limited space for the morphing structure and actuation mechanism due to the size of the wingtip.

The airfoil thickness at the wingtip is very low in the current aircraft, which constrains the design of the morphing structures and actuation systems.

2) The weight increase at the wingtip should be constrained to prevent any side effects, especially the aeroelasticity effects.

Nevertheless, morphing wingtip devices are worth investigating as a representative and promising morphing concept. The findings on the following three aspects are developed in this thesis:

a) Develop a useful morphing strategy for the morphing winglet.

Although different morphing concepts have been introduced, finding a suitable 
morphing strategy is still a meaningful investigation. The definition of the morphing variables is the first step for the analysis, where the design space can be explored.

b) Design and analysis of a proper morphing structure for the corresponding morphing winglet

A reliable morphing structure is the foundation of a practical morphing aircraft. Considering the limited space and existing morphing designs, an innovative concept will be proposed and verified.

c) Evaluate the morphing design at the system level

The final intention of the thesis is to find whether a morphing aircraft could be realised in the near future. Morphing aircraft needs to be evaluated on the system level, which takes the pros and cons of the morphing design into account.

\subsection{Dissertation outline}

In this thesis, Chapter 1 reviews the development of morphing aircraft, with the focus on morphing wingtip devices. A summary is made from the historical perspective, and the challenges in the field are listed and briefly discussed. Some well-known morphing projects are introduced, together with some background on compliant structures. The discussion is then extended to the motivation of the research, which emerges from the compromise of the system level benefit and the ambition to demonstrate a promising morphing design.

Chapter 2 starts the research with a case study, in which a variety of aerodynamic analyses are performed to show the potential benefits that a morphing winglet can bring. A baseline aircraft is modelled, and with different load cases, parametric studies are conducted to find the influence of different morphing variables, which implies the critical morphing variable required for the next stage of the research.

In Chapter 3, a compliant structure based on unsymmetrical stiffness is introduced and investigated at the conceptual level. The compliant structure can induce a rotation from a linear actuation, which can change the dihedral angle of the 
morphing winglet. A thermal analogy is presented to introduce the concept, and then a simplified 2-dimensional (2D) model is built. The influence of the unsymmetrical stiffness on the capability of carrying loads and inducing rotation is highlighted. The potential benefits of this novel concept are preliminarily estimated in a case study.

Chapter 4 investigates the equivalent modelling of corrugated panels, which will be used to provide the stiffness asymmetry. The equivalent model takes the extension and bending coupling into account, and the stiffness matrix is built by calculating the deflections of the corrugated panels. Detailed finite element analysis and tensile test of the corrugated panels are performed to verify the model.

The optimisation of the compliant structure is performed in Chapter 5. With the equivalent model of the corrugated panels, the stiffness matrix of the compliant structure is assembled, which is verified by commercial finite element software and experiments. The geometry parameters of the compliant structure and the corrugated panels are optimised to find the minimum actuation force to achieve the required deformation. The application of multiple units of compliant structures is also investigated, and the baseline design used in Chapter 3 is compared again with the optimum results.

A demonstration model based on the compliant structure is designed, analysed, manufactured and tested in Chapter 6. Optimisation of the compliant structure is employed to integrate the device into the airfoil, and solutions for the leading and trailing edges are also developed using flexible honeycomb structures. Experiments are conducted to show the static morphing capability, as well as the change of the aerodynamic effects in the wind tunnel tests.

Chapter 7 summarises the contributions of the thesis and discussion the future research directions. 


\section{Chapter 2 Aerodynamic benefits of morphing winglets---a case study}

\subsection{Background, model definition and tools}

In this section, an aerodynamic analysis is implemented to find the influence of a shape-changing winglet. Although the review of morphing winglets has highlighted the potential benefits, quantitative research is essential for the further detailed investigation and optimisation.

The aerodynamic analysis at the conceptual design stage is an approximate process that can affect the final solution significantly. Since the feasibility of a promising morphing aircraft remains an open question, access to fast modelling and analysis can help to fully explore the design space, as well as evaluate the different possibilities. Thus, the aerodynamic tool should suit the conceptual design level with a low calculation time, and capabilities for parametric modelling.

From the viewpoint of the structural aspect, the aerodynamic analysis provides the external loads for the structural design and analysis. While the main body of the thesis is focused on the structure of the morphing winglet, the aerodynamic loads will be required for the structural analysis.

A typical aircraft is selected for the aerodynamic analysis. The chosen baseline aircraft should be representative, and the geometry and weight data should be readily available. A single-aisle commercial airliner is selected since this kind of aeroplane is prevalent, and their span might be constrained by airport restrictions. Table 2.1 provides the main parameters of the airliner from open websites [101, 102], and the wing planform is shown in Figure 2.1. While the specific values may differ from those of the real-world, they can still provide a reasonable baseline to demonstrate the effects of the morphing winglet, since the primary interest is the change in 
performance caused by the morphing winglet. It should be noted that the reference geometry used in the aerodynamic analysis corresponds to the planar wing configuration when the winglet dihedral angle is 0 degrees.

\begin{tabular}{|c|c|c|c|}
\hline \multicolumn{2}{|c|}{ Geometry data } & \multicolumn{2}{c|}{ Weight data } \\
\hline Leading edge sweep angle & $28^{\circ}$ & Max take-off weight & $79000 \mathrm{~kg}$ \\
\hline Reference span & $36.56 \mathrm{~m}$ & Max zero fuel weight & $62000 \mathrm{~kg}$ \\
\hline Reference wing area & $131.61 \mathrm{~m}^{2}$ & Max landing weight & $66000 \mathrm{~kg}$ \\
\hline Wing root chord & $6.06 \mathrm{~m}$ & Max payload & $20000 \mathrm{~kg}$ \\
\hline Wingtip chord & $1.68 \mathrm{~m}$ & Fuel Capacity & $21000 \mathrm{~kg}$ \\
\hline
\end{tabular}

Table 2.1 Main parameters of the aircraft in the case study

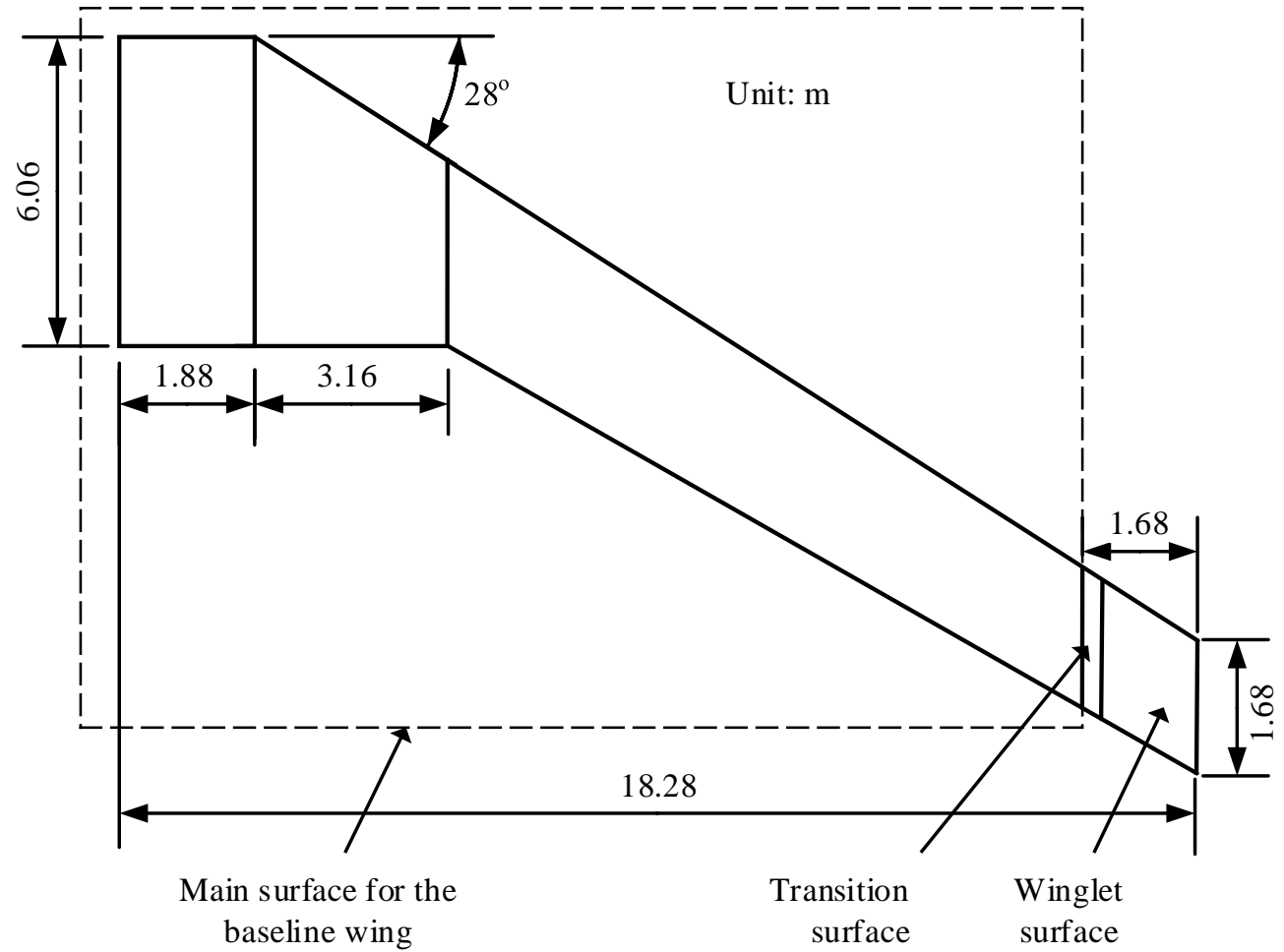

Figure 2.1 Sketch of the half wing layout used in Chapter 2 (Geometry parameters taken from [101], [102] ) 
The winglet has the same leading edge sweep angle as the wing, and its chord and length (semi-spanwise) are the same as the wing tip chord. Since the winglet can be regarded as a wing, any state-of-the-art morphing wing technology could theoretically be used on the winglet. However, too many morphing variables will make the winglet too complicated, and reduce the focus of the research.

Four variables are proposed in this Chapter, as shown in Figure 2.2, namely, the winglet dihedral angle, $\theta_{d}$, the winglet toe angle, $\theta_{t}$, the winglet twist angle, $\theta_{w}$ and the deflection angle of the winglet control surface, $\theta_{c}$. The control surface is modelled as a flap starting from $80 \%$ of the chord, while a compliant camber morphing structure could be also employed if preferred.
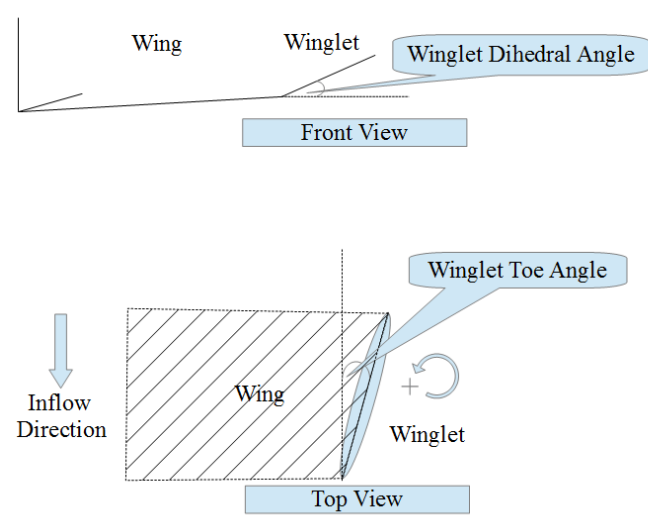
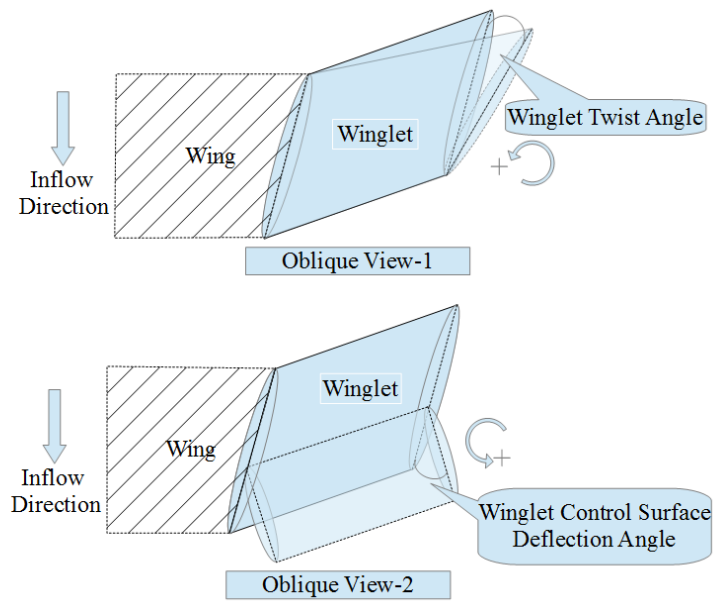

Figure 2.2 Morphing variables of the winglet

The aerodynamic analysis is performed using the open software AVL [103]. AVL is based on the vortex lattice method, and thus the results can be obtained within a short calculation time and acceptable accuracy for the conceptual study.

As a software based on the vortex lattice method, AVL calculates the induced drag but takes the parasitic drag as a fixed parameter. In the current study, the parasitic drag is calculated based on the concept of 'equivalent skin friction' [104], and remains constant since the change of wetted area of the winglet is small compared to the wetted area of the entire aircraft. The parasite drag coefficient is calculated as 


$$
C_{D_{0}}=C_{f e} \frac{S_{w e t}}{S_{r e f}}
$$

Here, the coefficient $C_{f e}$ is the equivalent skin friction coefficient, which is 0.003 according to [104]. $S_{\text {wet }}$ is the wetted area of the aircraft including the wing, the fuselage and the tail and $S_{r e f}$ is the reference area.

The parasite drag coefficient remains at 0.0206 in the calculation. And the drag coefficient $C_{D}$ is the sum of the parasitic drag and the induced drag as

$$
C_{D}=C_{D_{0}}+C_{D_{i}}
$$

It should be noted that during cruise the flight speed will reach Mach 0.78, which could lead to differences between the numerical model and the real-world situation, although AVL adopts a subsonic Prandtl-Glauert compressibility treatment [103]. Since the focus of this chapter is to show the influence of different morphing variables, the results should still be reasonable.

Another issue that could affect the accuracy is the number of significant figures in the AVL output. When the shape change is too small, the same aerodynamic performance can be obtained with different geometries. However, considering the focus of the research is to identify the morphing variable, which has the potential to introduce significant benefits, AVL is still a good option for the conceptual study.

The fuselage of the aircraft is simplified to a vertical plate generating no forces, and a convergence study was performed to determine a suitable number of vortices, which leads to 12 vortices in the chordwise direction and 34 vortices in the semi-spanwise direction. In AVL, the 'surface' concept is used to model a separate aerodynamic component. The current model uses one surface for the wing, one surface for the vertical tail and another one for the horizontal tail. An additional surface is created to model the winglet, while a small transition surface is also built between the wing and the winglet so that the toe angle of the winglet can be modelled separately without the influence of the twist angle of the wing. This transition surface may also represent the transition region in the structure to install 
the morphing structure and actuation system. In this chapter, the length (semi-spanwise) of the transition surface accounts for $10 \%$ of the winglet length (semi-spanwise), which is considered to be small enough to neglect its aerodynamic influence.

The original AVL uses input and output files to build the geometry and output results, which provides excellent access to its pre/post-processing. For parametric modelling and analysis, AVL is integrated with Matlab® [105]. Since the calculation results are stored in Matlab, the further data analysis is also more convenient, for example to obtain the aerodynamic loads from the initial outputs. Figure 2.3(a) shows the workflow in Matlab. The geometry file is generated by Matlab according to the data in Table 2.1, and then the flight conditions are set to start the analysis job in AVL. When the job finishes, the results will be read. Different types of jobs in AVL can be completed, such as to obtain the lift and drag coefficients, the wing root bending moment and the aerodynamic loads. Figure 2.3(b) shows an example of the pre-processing (geometry model) and the post-processing (the aerodynamic loads calculated in AVL) both plotted in Matlab.
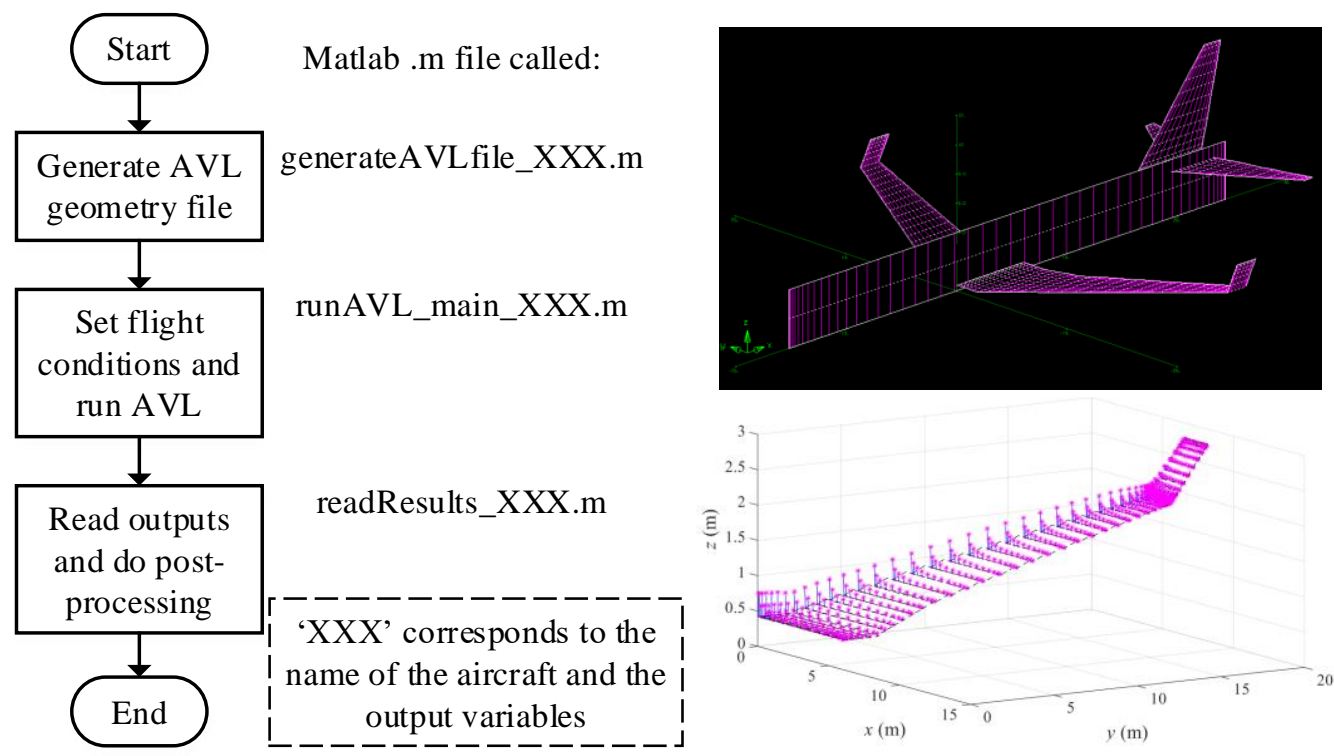

Figure 2.3 (a) AVL process step in Matlab (Left), (b) Pre-process example: geometry model in AVL (Right upper), (c) Post-process example: Aerodynamic loads on the wing (Right lower) 


\subsection{Parametric study of the morphing variables}

\subsubsection{Take-off}

The integration of AVL into Matlab allows for the parametric study of the geometry variables under different flight conditions. The flight envelope of an airliner consists of different phases, e.g. take-off, climbing, cruise, descending, holding and landing. To show the benefits of the morphing winglet under different flight conditions, different performance criteria are chosen for these phases, as shown in Table 2.2. The weights during different phases are determined by the weight data of the aircraft and considering the fuel consumption. The holding phase occurs after the cruise phase, and corresponds to a reduced weight.

\begin{tabular}{|c|c|c|c|}
\hline Phase & Take-off & Cruise & Holding \\
\hline Flight & Altitude: 0 & Angle of attack \\
conditions & $($ AOA $): 12^{\circ}$ & Veltitude: $12500 \mathrm{~m}$ & $\begin{array}{c}\text { Altitude: } 4572 \mathrm{~m} \\
\text { Velocity: } 102.8 \mathrm{~m} / \mathrm{s}\end{array}$ \\
& Weight: $78000 \mathrm{~kg}$ & Weight: $70000 \mathrm{~kg} / \mathrm{s}$ & $\begin{array}{c}\text { Weight: } 61000 \mathrm{~kg} \\
\text { Bank angle: } 20^{\circ}\end{array}$ \\
\hline Performance & Lift coefficient & Lift to drag ratio & Drag coefficient \\
criterion & $\left(C_{L}\right)$ & $(L / D)$ & $\left(C_{D}\right)$ \\
\hline
\end{tabular}

Table 2.2 Performance criteria

During the take-off phase, the horizontal distance of the transition point, $s_{a}$, is affected by the lift coefficient $C_{L}$. According to [106],

$$
\begin{aligned}
& S_{a}= \begin{cases}\left(2 R_{c} h_{s c}\right)^{1 / 2} & \gamma_{c}>\left(2 h_{s c} / R_{c}\right)^{1 / 2} \\
\frac{1}{2} \gamma_{c} R_{c}+\frac{h_{s c}}{\gamma_{c}} \gamma_{c}<\left(2 h_{s c} / R_{c}\right)^{1 / 2}\end{cases} \\
& R_{c}=\frac{2 W}{\rho g \Delta C_{L} S_{w}} \\
& \Delta C_{L}=\frac{\left(n_{g}-1\right) W}{(1 / 2) \rho V^{2} S_{w}}=C_{L}-\frac{W}{(1 / 2) \rho V^{2} S_{w}}
\end{aligned}
$$


where $R_{c}$ is the radius of the climbing circular phase, $h_{s c}$ is screen height, $\gamma_{c}$ is climbing angle achieved after the transition, $W$ is the aircraft weight, $n_{g}$ is the load factor, $\rho$ is the air density, $g$ is the acceleration due to gravity, $S_{w}$ is the wing area, and $V$ is the flight speed.

A higher lift coefficient can lead to a smaller $R_{c}$, which eventually reduces the transition distance during take-off, and increases the airport capability. The winglet dihedral angle is fixed at 90 degrees to reduce the total wing span since the airliner is still in the region of the airport. A representative angle of attack (AOA) is selected at 12 degrees, and the flap is deployed. The parametric study is conducted to show the effects of following morphing variables: winglet toe angle $\theta_{t}$, winglet twist angle $\theta_{w}$ and deflection angle of the control surface on the winglet $\theta_{c}$.

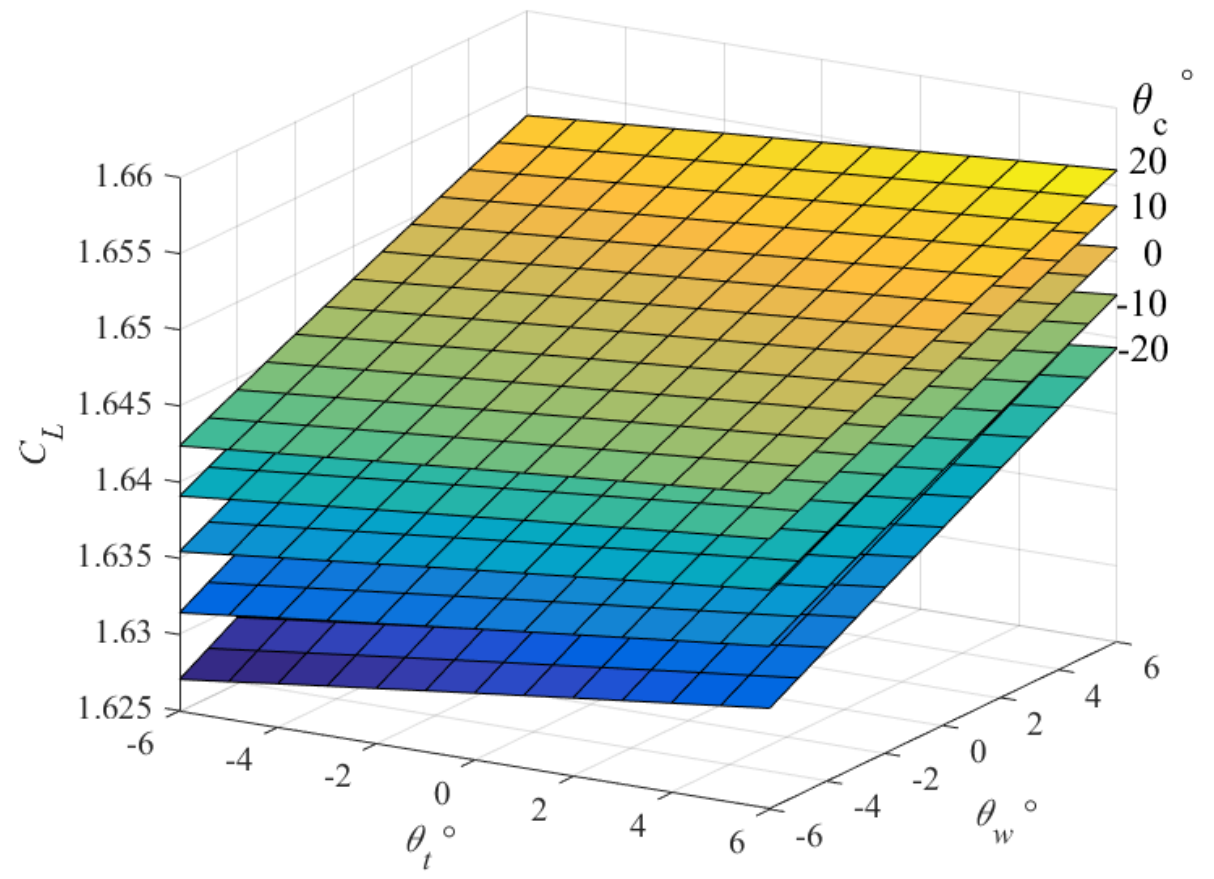

Figure 2.4 Lift Coefficient vs morphing variables during take-off

Figure 2.4 shows the relationship between the lift coefficient and the morphing variables. The relationship is quite linear since increases in the toe and twist angles will lead to an increase in the incidence angle of the winglet, which generates a higher lift coefficient. The deflection of the winglet control surface can also make a difference to the lift coefficient, in which a positive deflection angle works like a 
high lift system.

While a more significant lift coefficient is helpful during take-off, it also usually leads to a more substantial bending moment at the wing root, which can increase the structural weight. The wing root bending moment output by AVL is normalised by the dynamic pressure, the reference span and the reference wing area. Figure 2.5 shows that the morphing variables have a similar relationship with the bending moment or lift, which means the maximum bending moment occurs at the maximum lift. Furthermore, the negative deflection angle of the winglet control surface can give a smaller bending moment, which may provide a meaningful approach to alleviating the aerodynamic loads on the wing structure.

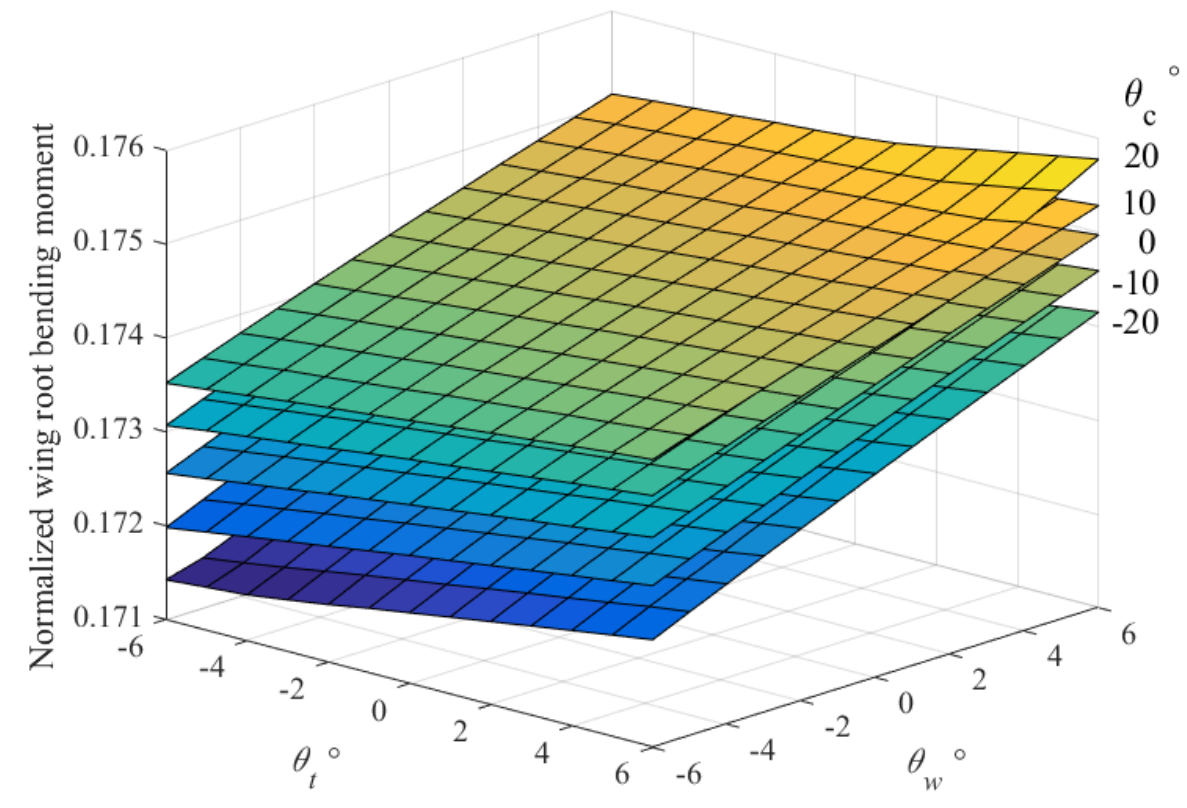

Figure 2.5 Normalised wing root bending moment vs morphing variables during take-off

\subsubsection{Cruise}

As an airliner, one of the most important potential benefits could be saving fuel, or increasing the range using the same amount of fuel. The range can be calculated as [104] 


$$
\begin{aligned}
& d R_{a}=-\frac{V}{C}\left(\frac{L}{D}\right) \frac{1}{W} d W \\
& R_{a}=\int_{W_{i}}^{W_{f}} d R_{a}=\frac{V}{C}\left(\frac{L}{D}\right) \ln \left(\frac{W_{i}}{W_{f}}\right)
\end{aligned}
$$

where $W$ and $C$ are the weight and the specific fuel consumption. The range is obtained by integrating from the initial weight $\left(W_{i}\right)$ to the final weight $\left(W_{f}\right)$. The lift to drag ratio, $L / D$, is analysed as the criterion for optimisation.

The following parametric analysis illustrates the effects of the morphing variables when the airliner flight is trimmed to maintain steady level flight. During this phase, the deflection angle of the winglet control surface will remain at zero, since deploying the control surface will cause additional drag and may damage the control surface. Thus, the morphing variables investigated are the winglet toe angle $\theta_{t}$, the winglet twist angle $\theta_{w}$ and the winglet dihedral angle $\theta_{d}$.

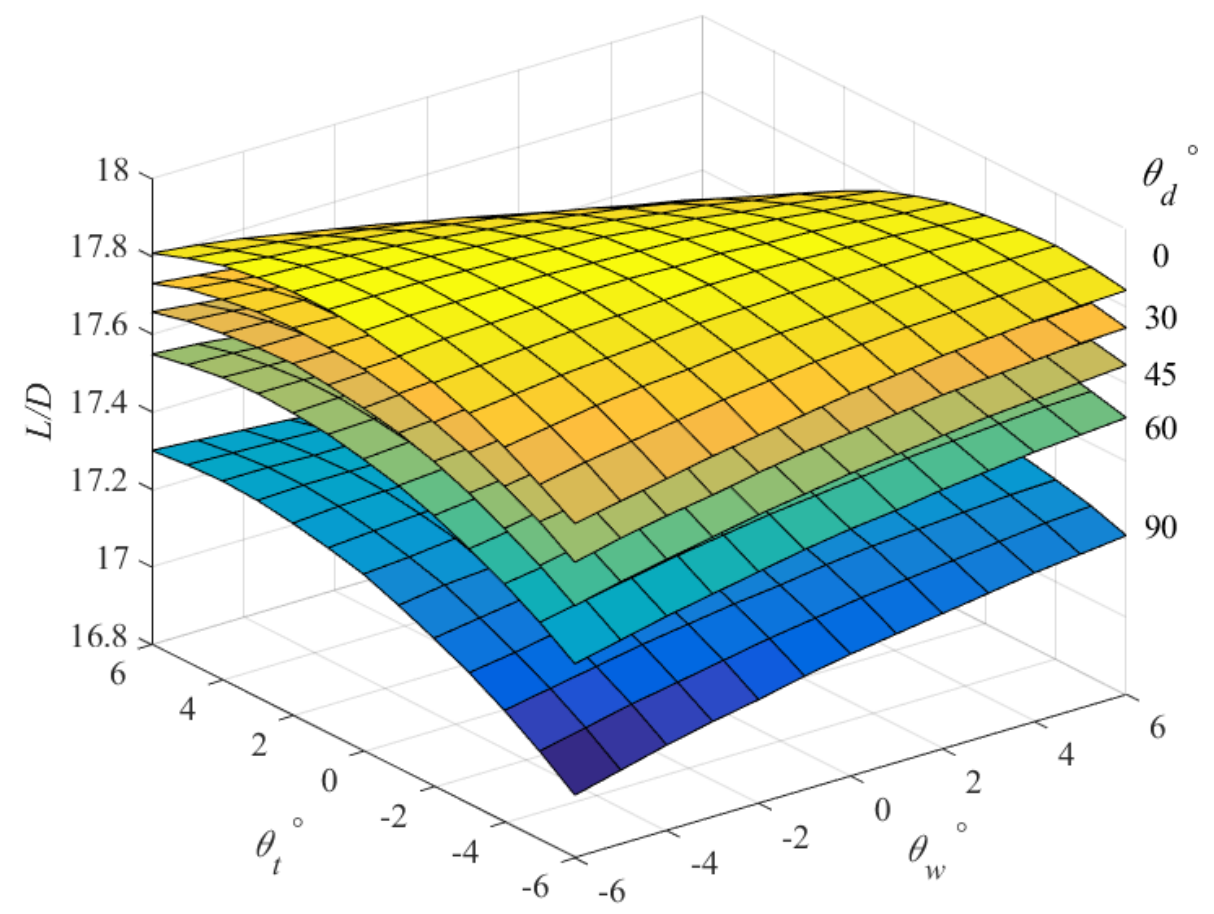

Figure 2.6 Lift to drag ratio vs morphing variables during cruise

As shown in Figure 2.6, the dihedral angle plays a vital role in determining the lift to drag ratio. It is straightforward to understand this effect since the wing span increases when the dihedral varies from $90^{\circ}$ to $0^{\circ}$, and a larger wing span generally increases the lift to drag ratio. The maximum lift to drag ratio is obtained when the 
toe angle is 2 degrees, and the twist angle is -6 degrees.

The relationship between the wing root bending moment and the morphing variables is still quite linear, as shown in Figure 2.7. The largest bending moments are found when the toe and twist angle are maximum, corresponding to the maximum angle of incidence of the winglet.

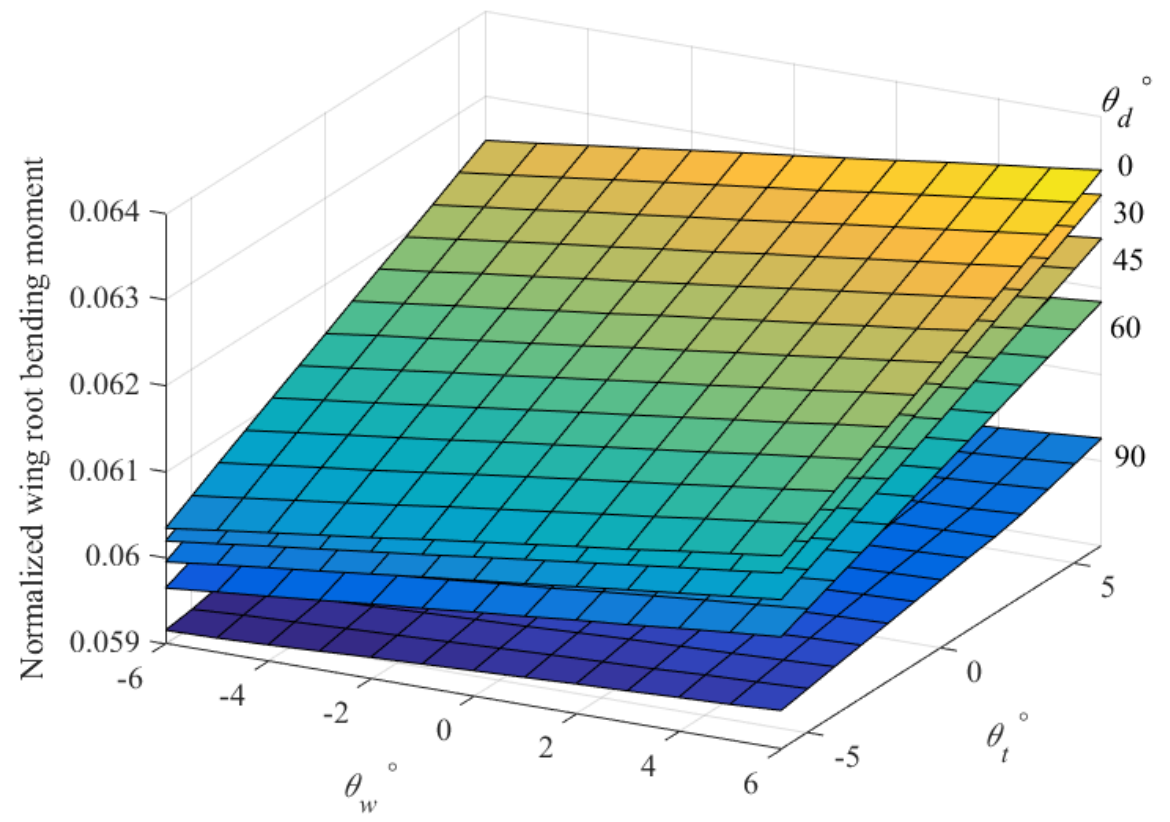

Figure 2.7 Normalised wing root bending moment vs morphing variables during cruise

\subsubsection{Holding}

The aircraft is trimmed to maintain a steady banked flight in the horizontal plane. The control surface on the winglet is not deployed, and the flight of the aircraft can be described as

$$
\begin{aligned}
& L \sin \beta_{b}=m \frac{V^{2}}{R_{t}} \\
& L \cos \beta_{b}=W \\
& D=T_{h r}=-\frac{W}{C}
\end{aligned}
$$

where $L$ is the lift, $D$ is the drag, $T_{h r}$ is the thrust, $\beta_{b}$ is the bank angle, $m$ is the aircraft mass, $R_{t}$ is the aircraft turn radius, and $\stackrel{\square}{W}$ is the rate of change of the aircraft 
weight. To reduce the fuel consumed, the drag should be reduced. Thus, the drag coefficient $C_{D}$ is analysed as the performance criterion, together with the wing root bending moment as a structural constraint.

Figure 2.8 shows the relationship between the morphing variables and the drag coefficient. The dihedral angle still plays a significant role in reducing the drag coefficient. Figure 2.9 shows the wing root bending moment during holding, which again shows a linear relationship.

It should be noted that a fixed profile drag coefficient is added to each of the calculations. Some of the results may not differ from each other due to the limitation of the accuracy of AVL, but this aerodynamic analysis is sufficient for the conceptual level study.

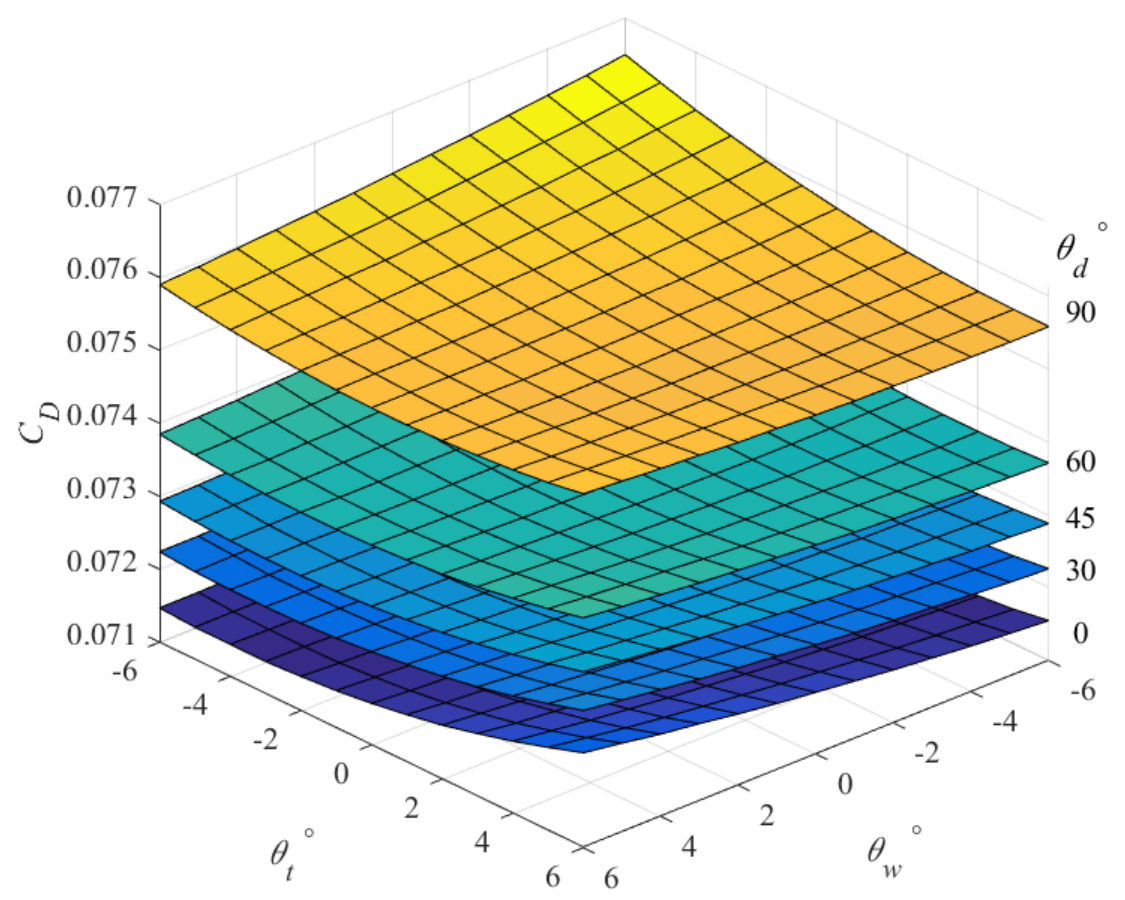

Figure 2.8 Drag coefficient vs morphing variables during holding 


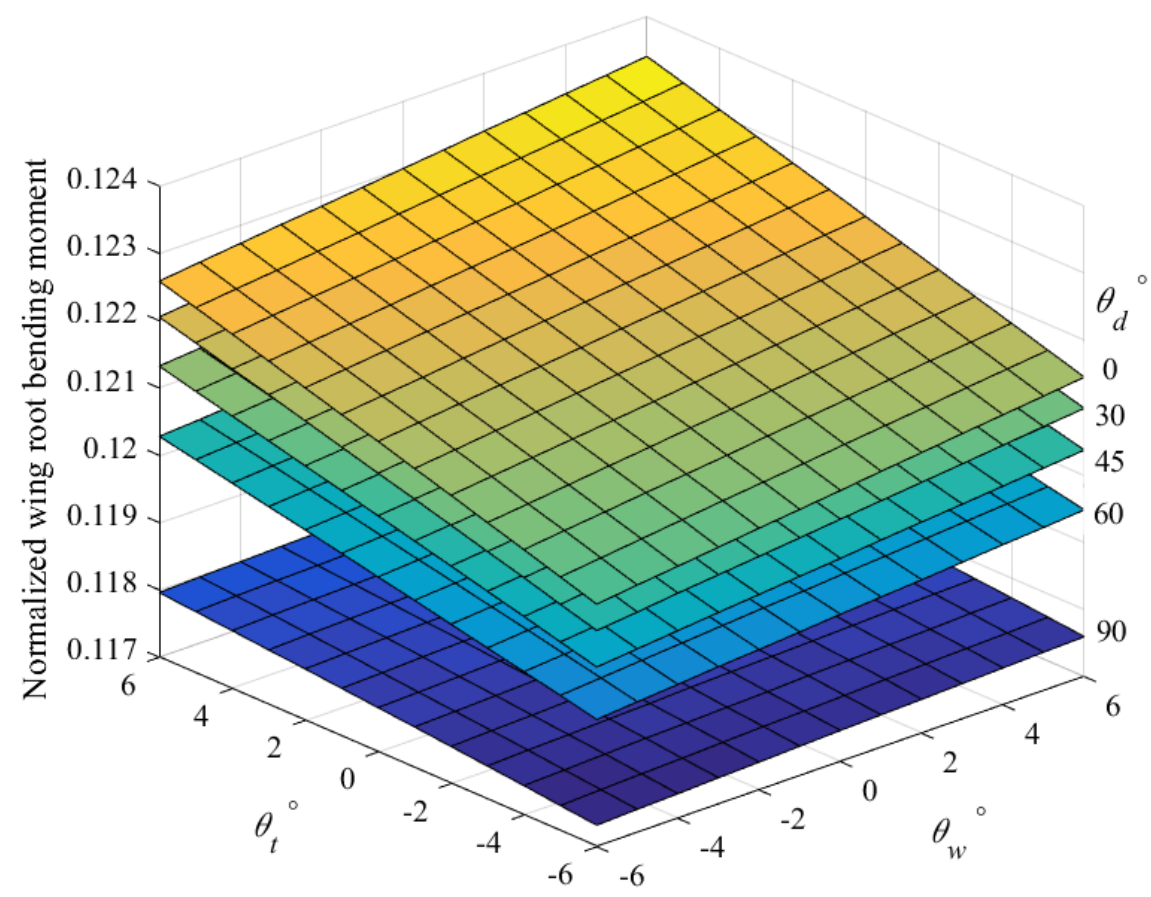

Figure 2.9 Normalised wing root bending moment vs morphing variables during holding

\subsection{Potential aerodynamic benefits}

The fixed shape winglet and the morphing winglet are compared to demonstrate the potential aerodynamic benefits. The shape of the fixed winglet is usually based on a high-fidelity aerodynamic analysis and wind tunnel tests.

It is not the subject of the current study to investigate the aerodynamic shape of the winglet using high-fidelity tools. Thus, a simple comparison is made according to the calculation results in the previous section at the conceptual design stage.

Since the wing span can be constrained by the airport, a fixed winglet has the winglet dihedral, toe, twist and deflection angles equal to 90, 2, -6 and 0 respectively, which can reduce the span with the maximum dihedral angle while giving a lift to drag ratio that is as large as possible. Concerning the morphing winglet, it can be seen that the most significant wing root bending moment occurs during the take-off phase. Thus, the dihedral angle can be set to zero when the airliner enters the cruise phase, to maximise the aerodynamic benefit while satisfying the structural 
constraints. The selected morphing winglet parameters during the different phases are summarised in Table 2.3.

\begin{tabular}{|c|c|c|c|}
\hline Phase & Take-off & Cruise & Holding \\
\hline Winglet Dihedral Angle $\left(^{\circ}\right)$ & 90 & 0 & 0 \\
\hline Winglet Toe Angle $\left(^{\circ}\right)$ & 6 & 2 & 2 \\
\hline Winglet Twist Angle $\left(^{\circ}\right)$ & 6 & -6 & -6 \\
\hline Winglet control surface deflection angle $\left(^{\circ}\right)$ & 20 & 0 & 0 \\
\hline
\end{tabular}

Table 2.3 Selected morphing variables during different phases

It should be emphasised that this evaluation is only for exploring the potential benefit that the morphing winglet might provide, which is purely conceptual and does not take the detailed design of the winglet into consideration.

The results in different flight phases are summarised in Table 2.4, in comparison to those of the fixed winglet. The weight starts from $78000 \mathrm{~kg}$ and ends at $62000 \mathrm{~kg}$, which represents the fuel consumed during the flight. Five points during the cruise phase are used to calculate the integral of the range as explained by Equation (2.2).

\begin{tabular}{|c|c|c|c|c|c|c|c|}
\hline Phase & Take-off & \multicolumn{5}{|c|}{ Cruise } & Holding \\
\hline Criterion & $C_{L}$ & \multicolumn{5}{|c|}{$L / D$} & $L / D$ \\
\hline Weight (kg) & 78000 & 78000 & 74000 & 70000 & 66000 & 62000 & 61000 \\
\hline $\begin{array}{c}\text { Morphing } \\
\text { winglet }\end{array}$ & 1.6560 & 17.8921 & 17.7582 & 17.5663 & 17.3145 & 16.9910 & 16.6100 \\
\hline $\begin{array}{c}\text { Fixed } \\
\text { winglet }\end{array}$ & 1.6435 & 17.2959 & 17.2035 & 17.0594 & 16.8515 & 16.5721 & 15.6638 \\
\hline
\end{tabular}

Table 2.4 Morphing winglet performance compared to a fixed winglet 
Table 2.4 shows that the lift to drag ratio with the morphing winglet remains larger than that with the fixed winglet. The advantage is caused by the unfolding of the winglet (reduced winglet dihedral angle), which leads to the larger lift to drag ratio.

Calculated using the data in Table 2.4, the range using a morphing winglet can be increased by about $2.97 \%$ compared to the fixed winglet, or $2.88 \%$ of fuel can be saved for the same range. The benefit is only calculated for the cruise phase; for a more complex flight envelope, where the holding and take-off phases are also considered, the overall benefits will be undoubtedly higher and more comprehensive.

\subsection{Summary}

The research on the morphing winglet starts with the aerodynamic analysis in this chapter. The aerodynamic model is built in AVL, which is based on the vortex lattice method, and integrated into Matlab for fast modelling and parametric study. The geometry information of the model comes from open access data and represents a typical narrow-body airliner. Four typical morphing variables are modelled to represent the shape change of a morphing winglet. Flight conditions, i.e., take-off, cruise and holding, are taken into consideration for their corresponding performance criterion.

The aerodynamic results imply that changing the dihedral angle of the winglet could have a direct impact on the aerodynamic criteria, and the structural loads on the wing root, both of which are critical factors to determine the aircraft performance. The straightforward influence confirms the essential requirement of the morphing structure: being able to fold the winglet or change its dihedral angle while carrying aerodynamic loads, which motivates the proposed morphing structure in the following chapters. 


\section{Chapter 3 Conceptual design of the morphing winglet}

\subsection{Compliant structure based on unsymmetrical stiffness}

\subsubsection{A thermal analogy}

In Chapter 2, the significant influence of the winglet dihedral angle has been highlighted. In this Chapter, a compliant structure is proposed to allow for the intended deformation by transferring linear actuation to rotation of the structure.

The approach is inspired by analogy to the bimetallic strip. Figure 3.1(a) shows the deformation of the bimetallic strip, which has different thermal expansion coefficients for the two metal strips, namely strip 1 and strip 2. As shown, the different thermal expansions can cause different axial displacements between strip 1 and strip 2. Supposing strip 1 has a larger displacement, the connection between strip 1 and strip 2 will force the bimetallic strip to bend, balancing the differential displacement. By using the unsymmetrical thermal properties in the two strips, mechanical deformation can be obtained when the bimetallic strip is heated. Substituting the unsymmetrical thermal expansion coefficient with the unsymmetrical axial stiffness, deformation can also be obtained when it is actuated.

Compared to the bimetallic strip, the proposed compliant structure consists of the upper beam, the lower beam and the connection beam between them, as shown in Figure 3.1(a). It should be noted that the terms 'upper' and 'lower' only mean the upper and lower position as shown in the figure, which do not represent their coordinates. The upper beam can have a smaller coordinate in the vertical direction if a coordinate is built in that way. The upper and lower beams can undergo extension deformation but have different axial stiffnesses. When the actuation force is applied, the differential axial deformation between the upper and lower beam will 
cause the rotation of the structure. This concept is demonstrated in Figure 3.1(b). Although the upper and lower beams are both represented by beam-type structures in Figure 3.1(a), different forms can be employed, such as the corrugation structure in Figure 3.1(b). Also, the connection beam should be strong and stiff enough to transfer the loads. In the demonstration, the sample introduces stiffness asymmetry by using different corrugation shapes and sizes in the upper and lower corrugation structures. The sample is clamped to simulate the fixed boundary condition, and the actuation force is applied onto the connection beam, under which an induced rotation of the structure can be observed from the red dashed lines.
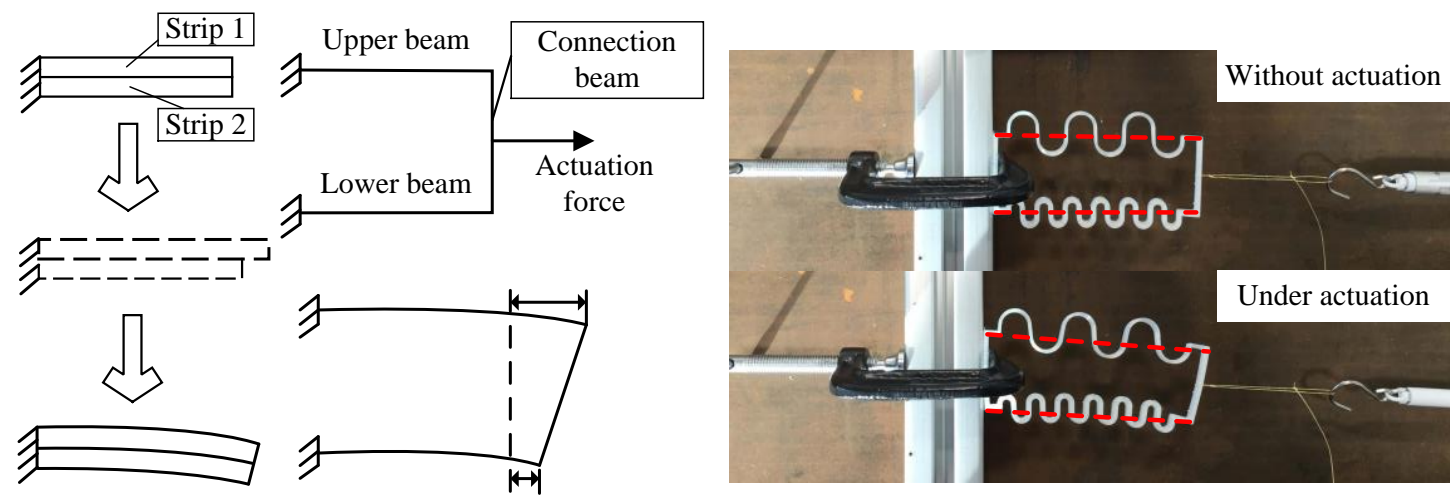

Figure 3.1 Concept of the compliant structure: (a) Analogy with the bimetallic strip,

(b) Rotation of a demonstration sample

\subsubsection{Simplified 2-dimensional model}

The proposed compliant structure is regarded as a frame, of which the beam members can both extend and bend, but will still follow the small deformation assumption. Figure 3.2 shows the 2-dimensional (2D) model that represents the deflection under external forces. The 2D model consists of the upper, lower and connection beams labelled $\mathrm{AB}, \mathrm{DC}$ and $\mathrm{BC}$ respectively. Beam $\mathrm{AB}$ and $\mathrm{DC}$ can undergo axial deformation and bend. Beam $\mathrm{BC}$ is assumed to be rigid compared to beams $\mathrm{AB}$ and $\mathrm{DC}$.

The Young's modulus, area and second moment of area of beams AB, DC and BC are represented by $E_{1}, A_{1}, I_{1}, E_{2}, A_{2}, I_{2}, E_{3}, A_{3}, I_{3}$ respectively. The length and height of the 2D model are represented by $a$ and $b$ respectively. In Figure 3.2 (a), the 
structure is actuated by the force $F$. To simplify the model, the actuation force is currently applied in the middle of beam $\mathrm{BC}$, namely point $\mathrm{E}$, which does not reduce the generality of the method.
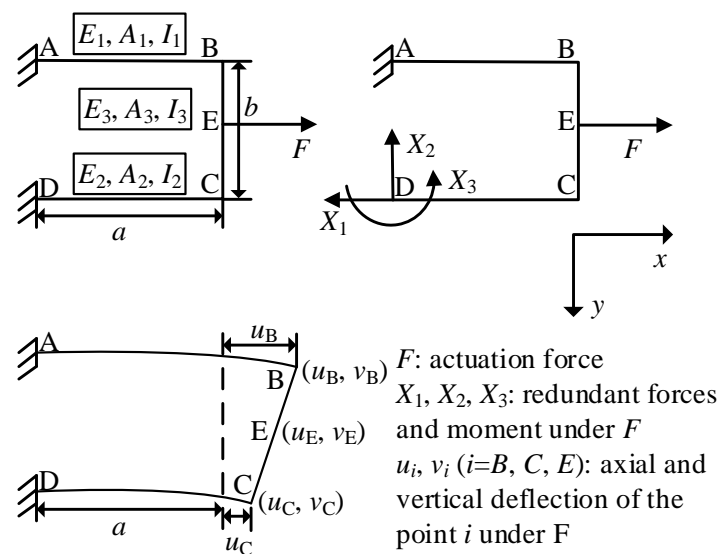
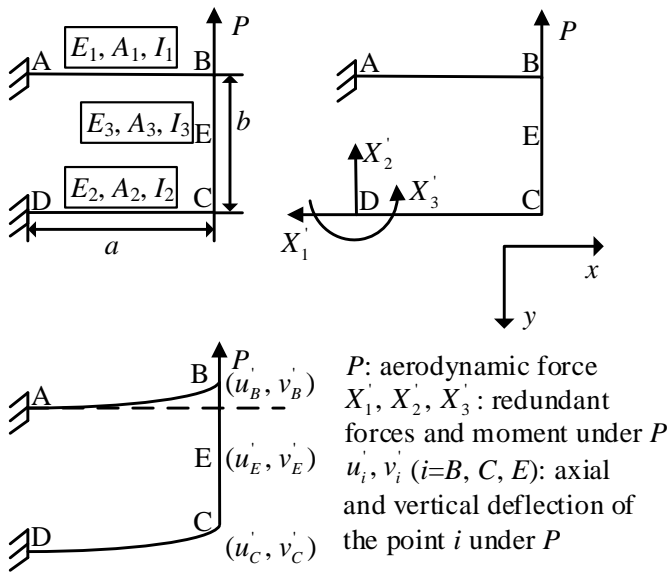

Figure 3.2 Deflection of the compliant structure: (a) under actuation force, (b) under aerodynamic force

Since the upper and lower beams are fixed at points A and D, there are six unknown reaction forces and the moments as shown in Figure 3.3, while only three independent equations of equilibrium are related to these variables. Thus the proposed compliant structure is statically indeterminate, and the deflection is calculated by the force method [107].
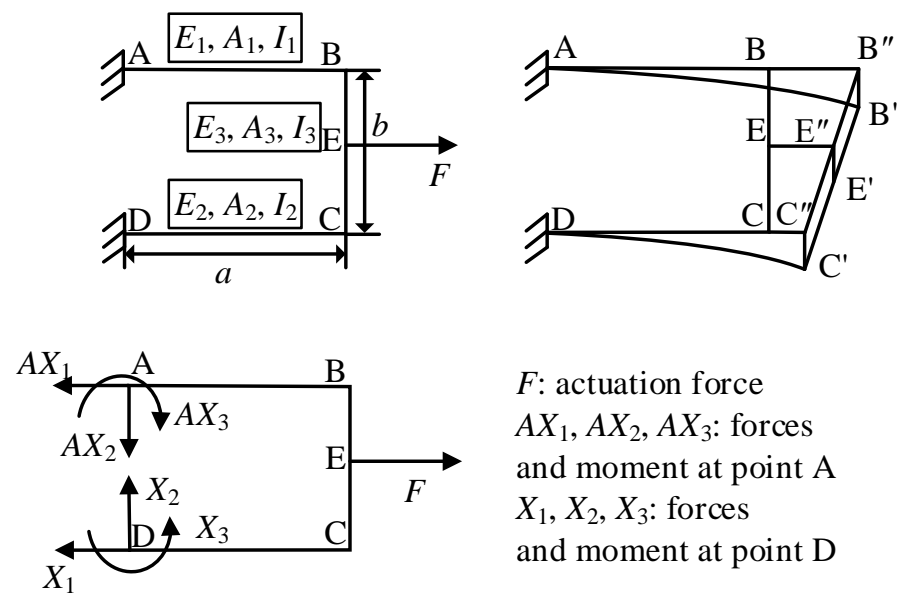

$F$ : actuation force $A X_{1}, A X_{2}, A X_{3}$ : forces and moment at point $\mathrm{A}$ $X_{1}, X_{2}, X_{3}$ : forces and moment at point $\mathrm{D}$

Figure 3.3 Loads at point A, D and the geometry relationship of point B, C, and E The reaction forces applied at point $\mathrm{D}$ are chosen as redundant reactions. Then the structure becomes statically determinate with $X_{1}, X_{2}, X_{3}$ applied at point D. To satisfy 
the original boundary condition, the redundant reactions need to make the horizontal displacement $u$, vertical displacement $v$ and rotation $\alpha$ at point $\mathrm{D}$ zero. Using the principle of superposition, $u$ can be expressed as

$$
u=u_{F}+u_{X_{1}}+u_{X_{2}}+u_{X_{3}}
$$

where $u_{F}$ is horizontal displacement caused by the actuation force $F$ alone, and $u_{X_{i}}(i=1,2,3)$ is the horizontal displacement caused by the reaction $X_{\mathrm{i}}$ alone.

According to the principle of virtual work, the displacement under an external load can be expressed as

$$
u=\sum \int \frac{m M}{E I} d x+\sum \int \frac{n N}{E A} d x
$$

where $M$ and $N$ are the bending moment and axial force under the external load, and $m, n$ are the bending moment and axial force under the unit load applied in the same direction as the required displacement. Figure 3.4 shows the internal bending moment and the axial force caused by $F$ and the unit load (bending moment and axial load starting from point $\mathrm{A})$, which is in the same direction to $X_{\mathrm{i}}(i=1,2,3)$. Thus, using the above equation, we obtain

$$
\begin{gathered}
u_{F}=\sum \int \frac{m M}{E I} d x+\sum \int \frac{n N}{E A} d x \\
=\int_{0}^{a} \frac{-b \cdot\left(\frac{1}{2} F b\right)}{E_{1} I_{1}} d x+\int_{0}^{a} \frac{-1 \cdot F}{E_{1} A_{1}} d x \\
=-\frac{F a}{E_{1} A_{1}}-\frac{F a b^{2}}{2 E_{1} I_{1}} \\
u_{X_{1}}=\int_{0}^{a} \frac{1 \cdot X_{1}}{E_{1} A_{1}} d x+\int_{0}^{a} \frac{1 \cdot X_{1}}{E_{2} A_{2}} d x+\int_{0}^{a} \frac{b \cdot b X_{1}}{E_{1} I_{1}} d x \\
=\left(\frac{a}{E_{1} A_{1}}+\frac{a}{E_{2} A_{2}}+\frac{a b^{2}}{E_{1} I_{1}}\right) X_{1}
\end{gathered}
$$

The same method can be used to obtain the other components of $u$, as well as the vertical displacement $v$ and rotation $\alpha$. Finally, at point $\mathrm{D}$ we obtain 


$$
\begin{aligned}
& u=-\frac{F a}{E_{1} A_{1}}-\frac{F a b^{2}}{2 E_{1} I_{1}}+\left(\frac{a b^{2}}{E_{1} I_{1}}+\frac{a}{E_{1} A_{1}}+\frac{a}{E_{2} A_{2}}\right) X_{1}+\frac{a^{2} b}{2 E_{1} I_{1}} X_{2}-\frac{a b}{E_{1} I_{1}} X_{3} \\
& v=-\frac{a^{2} b}{4 E_{1} I_{1}} F+\frac{a^{2} b}{2 E_{1} I_{1}} X_{1}+\left(\frac{a^{3}}{3 E_{1} I_{1}}+\frac{a^{3}}{3 E_{2} I_{2}}\right) X_{2}-\left(\frac{a^{2}}{2 E_{1} I_{1}}+\frac{a^{2}}{2 E_{2} I_{2}}\right) X_{3} \\
& \alpha=\frac{F a b}{2 E_{1} I_{1}}-\frac{a b}{E_{1} I_{1}} X_{1}-\left(\frac{a^{2}}{2 E_{1} I_{1}}+\frac{a^{2}}{2 E_{2} I_{2}}\right) X_{2}+\left(\frac{a}{E_{1} I_{1}}+\frac{a}{E_{2} I_{2}}\right) X_{3}
\end{aligned}
$$

Making the displacement and rotation at point $\mathrm{D}$ zero, the redundant reactions can be solved to give

$$
\begin{aligned}
& X_{1}=\frac{E_{2} A_{2}\left(E_{1} A_{1} b^{2}+2 E_{1} I_{1}+2 E_{2} I_{2}\right)}{\left(E_{1}^{2} A_{1} I_{1}+E_{2}^{2} A_{2} I_{2}+E_{1} E_{2} A_{1} I_{2}+E_{1} E_{2} A_{2} I_{1}+E_{1} E_{2} A_{1} A_{2} b^{2}\right)} \frac{F}{2} \\
& X_{2}=0 \\
& X_{3}=-\frac{E_{2} I_{2}\left(E_{1} A_{1}-E_{2} A_{2}\right)}{\left(E_{1}^{2} A_{1} I_{1}+E_{2}^{2} A_{2} I_{2}+E_{1} E_{2} A_{1} I_{2}+E_{1} E_{2} A_{2} I_{1}+E_{1} E_{2} A_{1} A_{2} b^{2}\right)} \frac{F b}{2}
\end{aligned}
$$

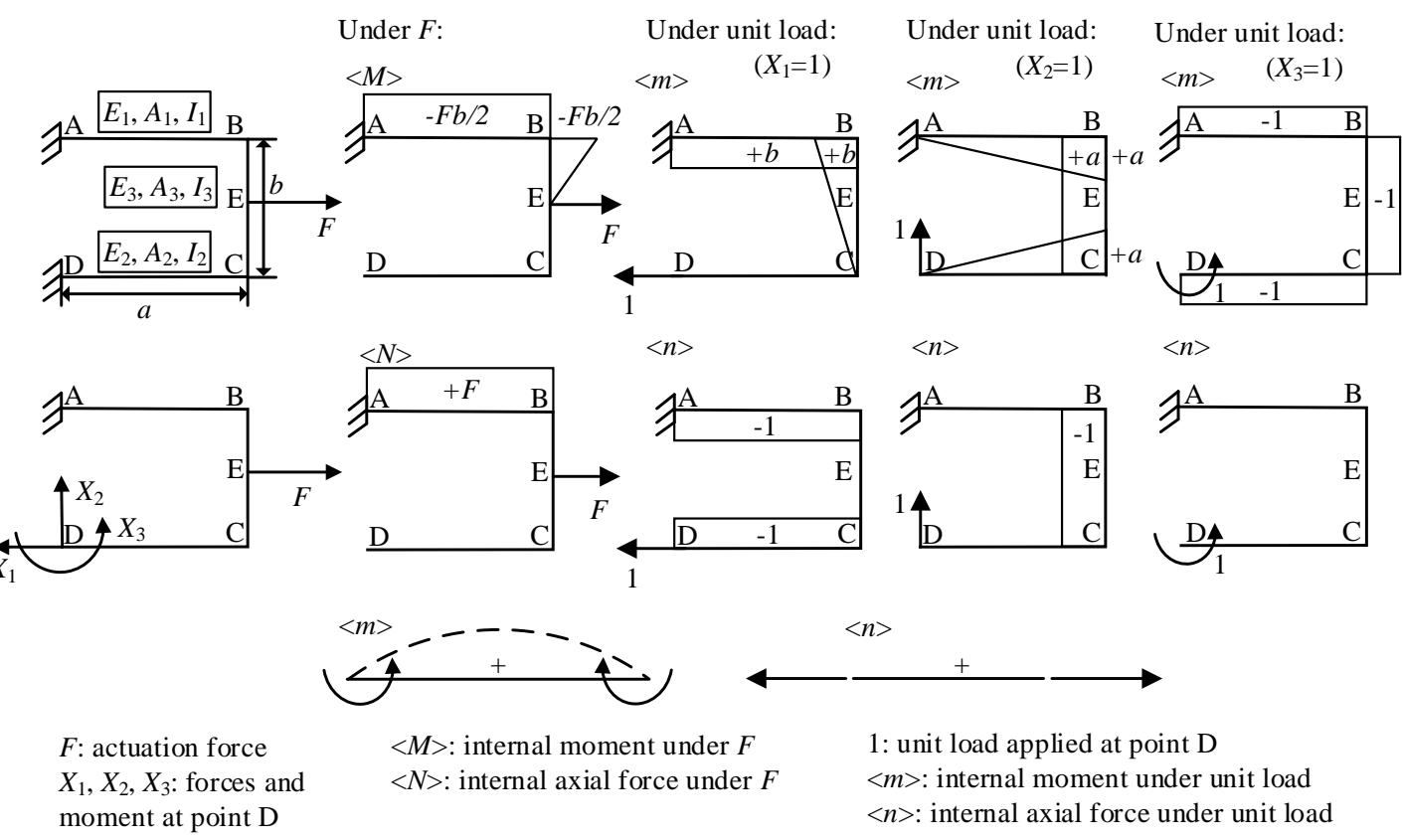

Figure 3.4 Internal loads under the actuation force $F$

Then, we can obtain the reactions at point $\mathrm{A}$ from the equilibrium equations of the compliant structure as shown in Figure 3.5 

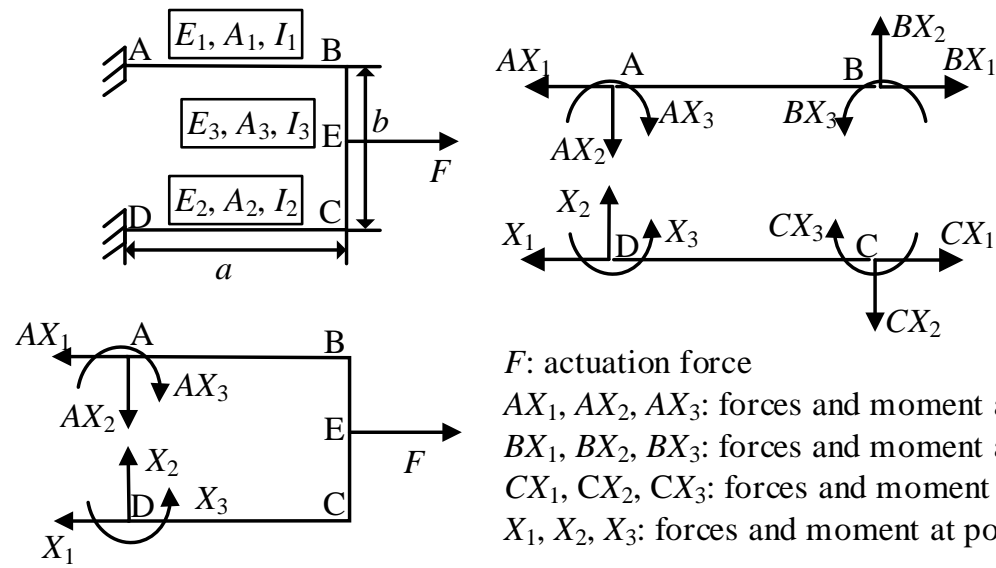

$F$ : actuation force

$A X_{1}, A X_{2}, A X_{3}$ : forces and moment at point $\mathrm{A}$ $B X_{1}, B X_{2}, B X_{3}$ : forces and moment at point $\mathrm{B}$ $C X_{1}, \mathrm{CX}_{2}, \mathrm{C} X_{3}$ : forces and moment at point $\mathrm{C}$ $X_{1}, X_{2}, X_{3}$ : forces and moment at point D

Figure 3.5 Balanced structure and beam $\mathrm{AB}$ and $\mathrm{DC}$

According to the equilibrium of the whole structure,

$$
\begin{aligned}
& F-A X_{1}-X_{1}=0 \\
& A X_{2}-X_{2}=0 \\
& A X_{3}+X_{1} b-\frac{1}{2} F b-X_{3}=0
\end{aligned}
$$

Thus,

$$
\begin{aligned}
& A X_{1}=\frac{E_{1} A_{1}\left(E_{2} A_{2} b^{2}+2 E_{1} I_{1}+2 E_{2} I_{2}\right)}{\left(E_{1}^{2} A_{1} I_{1}+E_{2}^{2} A_{2} I_{2}+E_{1} E_{2} A_{1} I_{2}+E_{1} E_{2} A_{2} I_{1}+E_{1} E_{2} A_{1} A_{2} b^{2}\right)} \frac{F}{2} \\
& A X_{2}=0 \\
& A X_{3}=\frac{E_{1} I_{1}\left(E_{1} A_{1}-E_{2} A_{2}\right)}{\left(E_{1}^{2} A_{1} I_{1}+E_{2}^{2} A_{2} I_{2}+E_{1} E_{2} A_{1} I_{2}+E_{1} E_{2} A_{2} I_{1}+E_{1} E_{2} A_{1} A_{2} b^{2}\right)} \frac{F b}{2}
\end{aligned}
$$

Applying the equations of equilibrium to the upper and lower beams separately gives the internal forces and moments at point $\mathrm{B}$ and $\mathrm{C}$, after which the displacements of point $\mathrm{B}$ and $\mathrm{C}$ can be obtained.

For the upper beam AB:

$$
\begin{gathered}
B X_{1}-A X_{1}=0 \\
A X_{2}-B X_{2}=0 \\
A X_{3}-B X_{3}-B X_{2} a=0 \\
u_{B}=B X_{1} a /\left(E_{1} A_{1}\right) \\
v_{B}=-B X_{3} a^{2} /\left(2 E_{1} I_{1}\right)-B X_{2} a^{3} /\left(3 E_{1} I_{1}\right)
\end{gathered}
$$

So, 


$$
\begin{aligned}
u_{B} & =\frac{a\left[F-E_{2} A_{2} F\left(E_{1} A_{1} b^{2}+2 E_{1} I_{1}+2 E_{2} I_{2}\right) /\left(2 K_{t}\right)\right]}{E_{1} A_{1}} \\
& =\frac{F}{2} a \frac{2 E_{1} I_{1}+2 E_{2} I_{2}+E_{2} A_{2} b^{2}}{K_{t}} \\
v_{B} & =\frac{a^{2} \frac{F b}{2}\left[E_{2} A_{2}\left(E_{1} A_{1} b^{2}+2 E_{1} I_{1}+2 E_{2} I_{2}\right) / K_{t}-1+E_{2} I_{2}\left(E_{1} A_{1}-E_{2} A_{2}\right) / K_{t}\right]}{2 E_{1} I_{1}} \\
& =\frac{F b}{2} a^{2} \frac{E_{2} A_{2}-E_{1} A_{1}}{2 K_{t}} \\
K_{t} & =E_{1} A_{1} E_{1} I_{1}+E_{2} A_{2} E_{2} I_{2}+E_{1} A_{1} E_{2} I_{2}+E_{1} I_{1} E_{2} A_{2}+E_{1} A_{1} E_{2} A_{2} b^{2}
\end{aligned}
$$

For the lower beam DC:

$$
\begin{aligned}
& C X_{1}-X_{1}=0 \\
& C X_{2}-X_{2}=0 \\
& C X_{3}+C X_{2} a-X_{3}=0 \\
& u_{C}=\frac{C X_{1} a}{E_{2} A_{2}} \\
& v_{C}=\frac{C X_{3} a^{2}}{2 E_{2} I_{2}}+\frac{C X_{2} a^{3}}{3 E_{2} I_{2}}
\end{aligned}
$$

Thus

$$
\begin{aligned}
& u_{C}=\frac{F}{2} a \frac{2 E_{1} I_{1}+2 E_{2} I_{2}+E_{1} A_{1} b^{2}}{K_{t}} \\
& v_{C}=\frac{F b}{2} a^{2} \frac{E_{2} A_{2}-E_{1} A_{1}}{2 K_{t}}
\end{aligned}
$$

Point $\mathrm{E}$ is the midpoint of beam BC. As shown in Figure 3.3, BB", CC", EE" are the horizontal displacements of points $\mathrm{B}, \mathrm{C}$ and $\mathrm{E}$ respectively, and B"B', E"E', C"C' are the vertical displacements of points B, C and E. According to the geometry relationship, 


$$
\begin{aligned}
u_{E} & =\frac{u_{B}+u_{C}}{2} \\
& =\frac{F}{2} a \frac{2 E_{1} I_{1}+2 E_{2} I_{2}+b^{2}\left(E_{1} A_{1}+E_{2} A_{2}\right) / 2}{K_{t}} \\
v_{E} & =\frac{v_{B}+v_{C}}{2} \\
& =\frac{F b a^{2}}{2} \frac{E_{2} A_{2}-E_{1} A_{1}}{2 K_{t}}==\frac{F b a^{2}}{4 K_{t} E_{1} A_{1}}\left(r_{s}-1\right)
\end{aligned}
$$

where the term $K_{t}$ is the combination of the axial and bending stiffness of both upper and lower beams, which can be expressed as

$$
K_{t}=E_{1} A_{1} E_{1} I_{1}+E_{2} A_{2} E_{2} I_{2}+E_{1} A_{1} E_{2} I_{2}+E_{1} I_{1} E_{2} A_{2}+E_{1} A_{1} E_{2} A_{2} b^{2}
$$

Moreover, the ratio $r_{s}$ is the axial stiffness ratio between the lower and upper beam as

$$
r_{s}=\frac{E_{2} A_{2}}{E_{1} A_{1}}
$$

According to Equation 3.15, no vertical deflection can be obtained when the ratio $r_{s}$ is equal to 1 , which means the upper and lower beam have the same axial stiffness.

To verify the expression, the analytical results are compared with the finite element method in the commercial software Abaqus ${ }^{\circledR}[108]$ as shown in Figure 3.6. In this case, the length, width and height of the structure are all equal to $0.1 \mathrm{~m}$ and the actuation force is $1000 \mathrm{~N}$. The mesh size is selected at $0.002 \mathrm{~m}$, and the model uses 7500 S4R general purpose shell elements. The general purpose shell element can simulate both thin and thick plates, and the use of the shell elements allows for the fast model building for future study of 3-dimensional cases by modifying the script files of the Abaqus ${ }^{\circledR}$ models.

At first, the induced displacement is calculated using aluminium beams but with different thickness. The upper beam has a rectangular profile, whose thickness is fixed at $1 \mathrm{~mm}$, while the thickness of the lower beam is increased. The analytical method is then compared to a finite element model of composite plates. The 
composite layup is symmetric, and the composite ply angle is 0 or 90 degrees in turn concerning the $x$-axis. The moduli $E_{1}$ and $E_{2}$ are obtained from the effective modulus in the $x$-direction of the composites. The composite in the upper part is made of 10 plies while the number of plies of the lower composite varies from 10 to 250 . Although 250 plies will make the composite too thick and unpractical, it will not influence the verification process. The composites properties are from reference [109], and the ply thickness is $0.125 \mathrm{~mm}$. The micromechanical models in [109] are used to calculate the elastic moduli and ultimate strength of the lamina. Although experiments should be performed to test the composites, the current approach provides reasonable material data for the conceptual level study. Table 3.1 provides the material properties used, and Table 3.2 summarises the lamina properties when the matrix volume fraction is 0.7 .

\begin{tabular}{|c|c|c|c|}
\hline \multicolumn{2}{|c|}{ Matrix properties (Epoxy) } & \multicolumn{2}{c|}{ Fiber properties (Fiber glass) } \\
\hline Axial modulus (GPa) & 3.4 & Axial modulus (GPa) & 85 \\
\hline Poisson's ratio & 0.3 & Poisson's ratio & 0.2 \\
\hline Shear modulus (GPa) & 1.308 & Shear modulus (GPa) & 35.42 \\
\hline Tensile strength (MPa) & 72 & Tensile strength (MPa) & 1550 \\
\hline Compressive strength (MPa) & 102 & Compressive strength (MPa) & 1550 \\
\hline Shear strength (MPa) & 34 & Shear strength (MPa) & 35 \\
\hline
\end{tabular}

Table 3.1 Properties of the matrix and fibre

\begin{tabular}{|c|c|c|c|}
\hline \multicolumn{2}{|c|}{ Elastic moduli (GPa) } & \multicolumn{2}{c|}{ Ultimate strength (MPa) } \\
\hline Axial modulus & 27.9 & Axial tensile strength & 501.84 \\
\hline Transverse modulus & 4.78 & Axial compressive strength & 98.0 \\
\hline Poisson's ratio & 0.27 & Transverse tensile strength & 41.17 \\
\hline Shear modulus & 1.84 & Transverse compressive strength & 58.26 \\
\hline & & In-plane shear strength & 19.36 \\
\hline
\end{tabular}

Table 3.2 Properties of the composite lamina

Figure 3.6 shows that the linear actuation applied at beam BC can induce the rotation 
of the structure. When the upper and lower beams have the same axial stiffness, no rotation is obtained and with the increase of the lower beam stiffness, the vertical deflection will increase first and then reduce under the same actuation force. The initial increase is due to the increased stiffness asymmetry while the deflection would be reduced inevitably since an infinite stiffness of the lower beam would lead to zero deflection. For the isotropic aluminium plate, the error between the analytical solution and the finite element method is smaller than $3 \%$, and for the composite plate, the error is smaller than $1 \%$, which means the $2 \mathrm{D}$ simplified model provides reasonable accuracy. It should also be noted that the example is only intended to verify the possibility of inducing the rotation due to the unsymmetrical stiffness. Although the vertical deflection demonstrated is quite small due to the large stiffness and the size of the structure, a larger deflection can be introduced by changing the stiffness of the structure.
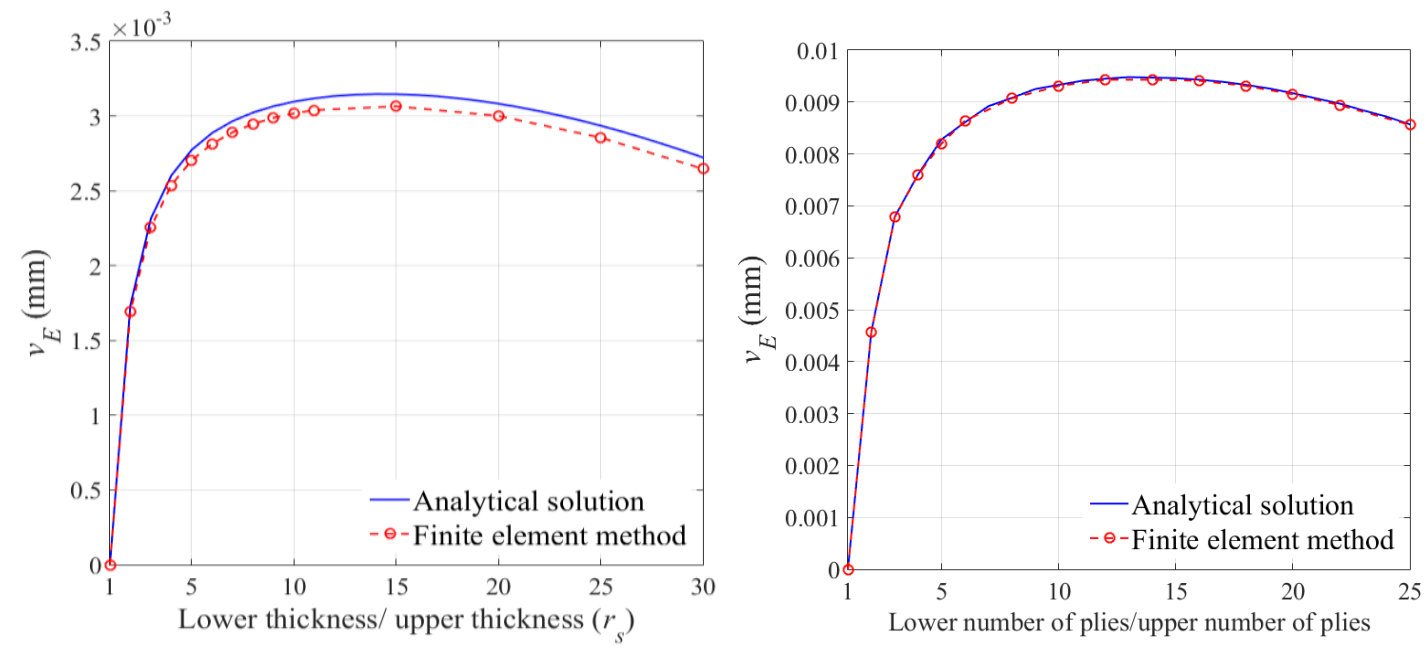

Figure 3.6 Actuated deflection of: (a) Aluminum structure, (b) Composite structure

As a morphing structure, the proposed compliant structure needs to change shape and maintain the geometry under aerodynamic loads simultaneously. The deformation under aerodynamic loads is calculated using the simplified model. As shown in Figure 3.2(b), the aerodynamic loads are concentrated at point B as a vertical force $P$. The displacement of point $\mathrm{E}$ under the aerodynamic force is obtained in the same way as the displacement of point $E$ under the actuation force as 


$$
\begin{aligned}
& u_{E}^{\prime}=\frac{E_{1} A_{1}-E_{2} A_{2}}{4 K_{t}} P a^{2} b \\
& v_{E}^{\prime}=\frac{\left(3 E_{1} A_{1} E_{2} A_{2} b^{2} / K_{t}-4\right)}{12\left(E_{1} I_{1}+E_{2} I_{2}\right)} P a^{3}
\end{aligned}
$$

In contrast to the actuation force case, the vertical deflection is not related to the difference in the axial stiffnesses in the upper and lower beams. The change in the vertical deflection under aerodynamic force will be different to that under actuation force if the stiffness asymmetry is increased, which allows the decoupling of the vertical deflections under actuation and aerodynamic forces.

\subsection{Influence of the unsymmetrical stiffness}

In this section, the influence of the unsymmetrical stiffness is shown. A detailed design of the upper and lower structure is necessary to determine the different stiffnesses in the compliant structure.

In this chapter, corrugated structures are used in the upper and lower beams with different composite properties. The corrugated structure has been investigated extensively for its application in morphing aircraft in recent years, such as $[32,110]$.

The corrugated structure has a low axial elastic modulus and a large deformation limit due to its anisotropic properties. By substituting the Young's moduli, $E_{1}$ and $E_{2}$, with the equivalent modulus in the corresponding direction, the deflection of the morphing structure can be calculated by an analytical approximation.

It should be noted that since the corrugated panel has a fixed boundary condition, the corrugated panel should be fixed carefully to eliminate the extension and bending coupling of corrugated panels, which will be further explained in Chapter 4 and 5. In this chapter, the equivalent modulus of the corrugated structure is obtained using the homogeneous method proposed in [111]. The round corrugation is used, and the geometry definition is shown in Figure 3.7(a). The corrugation structure is made of the composites described in Table 3.2. Detailed finite element models are created first to compare the equivalent modulus to the homogeneous method in Figure 3.7(b) 
and to obtain the deformation limit. The models are created in Abaqus®, and each finite element model has 4 round corrugations. The model is pinned at one end, and the axial displacement is applied at the other end, from which the reaction forces are obtained to calculate the equivalent modulus. The S4R shell elements are used and the mesh size is $0.001 \mathrm{~m}$ to ensure the geometry of the round corrugation is produced accurately. The results from the equivalent method have a small error (less than 1\%) compared to the detailed finite element analysis.
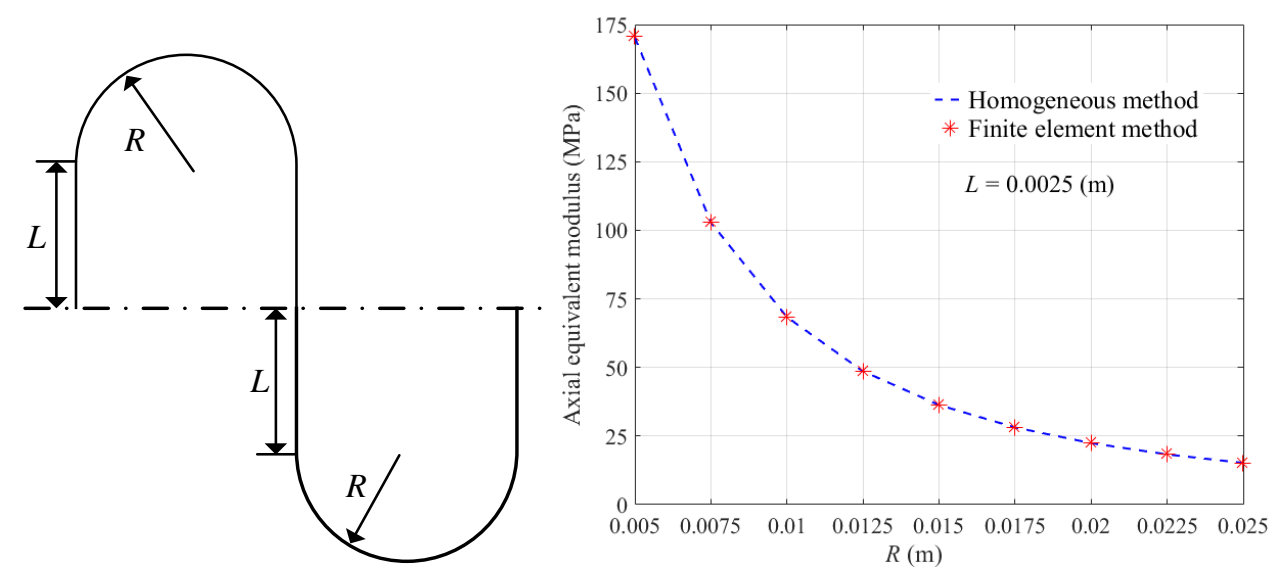

Figure 3.7 (a) Geometry of one round corrugation, (b) Equivalent modulus of the round corrugation

The stiffness asymmetry can be introduced by either changing the shape of the round corrugation, i.e. $L$ and $R$, or changing the thickness, or the number of plies and ply angle if composite material is used. To simplify the problem, the shape is fixed at $R$ $=0.025 \mathrm{~m}$ and $L=0.01 \mathrm{~m}$ in the following study, and the stiffness in the lower corrugation structure is changed by changing the number of plies while fixing the upper properties. The length, width and height of the compliant structure are all equal to $0.1 \mathrm{~m}$.

Parametric studies are conducted to show the influence of the unsymmetrical stiffness. To represent the shape change of the compliant structure, the rotation angle $\beta$ is calculated according to the displacements of point $\mathrm{E}$ as shown in Figure 3.8. 


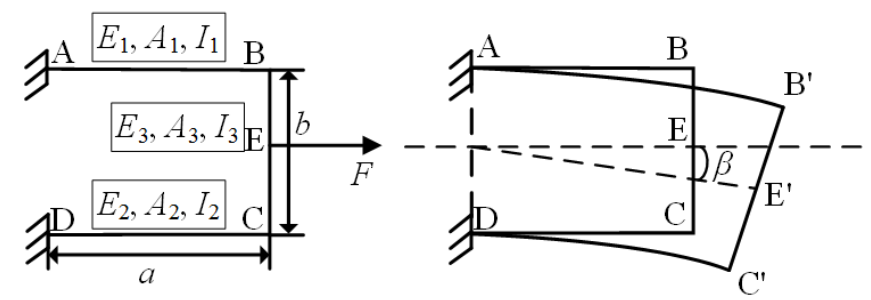

Figure 3.8 Rotation angle of the compliant structure represented by the displacement of point $\mathrm{E}$

Figure 3.9(a) shows the influence of the unsymmetrical stiffness on the rotation angle $\beta$. The aerodynamic force is fixed at $100 \mathrm{~N}$, and the rotation angles under different actuation forces are considered.

The simulated actuation and aerodynamic forces are representative and used to demonstrate the asymmetric stiffness concept; in a morphing application the estimated aerodynamic forces would be used, and the actuation force would be optimised. The $x$-axis represents the axial stiffness ratio between the lower and upper beam, $r_{s}$. With the increase of this ratio, both the total stiffness and stiffness asymmetry of the structure are increased. With the increase of the total stiffness, the rotation under aerodynamic force declines sharply while the rotation angle under actuation force will climb first. If enough stiffness is added to the lower corrugation, the rotation angle caused by the actuation force will be higher than that of the aerodynamic force.
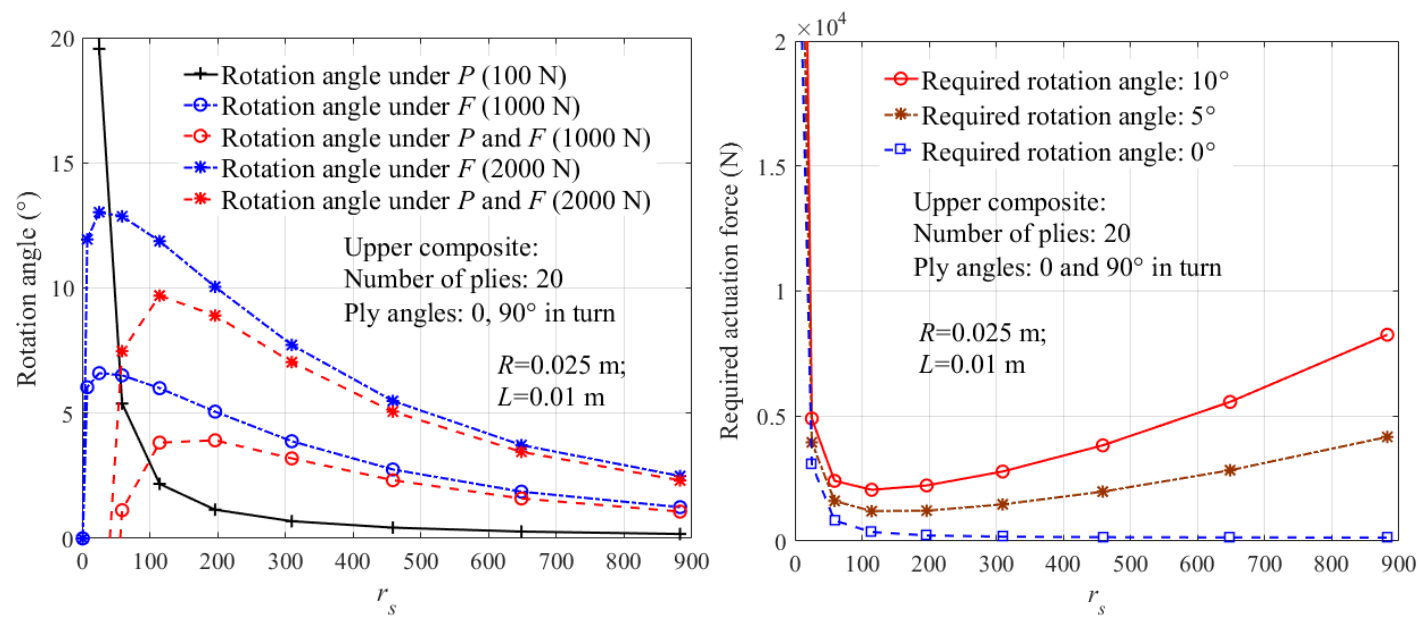

Figure 3.9 (a) Rotation angle under actuation force and aerodynamic force; (b)

Required actuation force for the target rotation angle 
From another perspective, the required angle, which is the rotation angle for a specific aerodynamic force, determines the required actuation force. Different to the conventional structure the actuation force actively takes part in determining the structure's geometry. As shown in Figure 3.9(b), different actuation forces are required for different required angles although the aerodynamic force is fixed at $100 \mathrm{~N}$. When the axial stiffness ratio, $r_{s}$, is too small, the total stiffness of the structure will be so small that the required angle cannot be achieved. If the required angle is 0 , which means the actuation force is only used to maintain the geometry under aerodynamic force, the required actuation force will be reduced continuously by increasing the stiffness in the lower part. However if the required angle is larger than 0 , the actuation force will climb again after the initial decline. Since both the stiffness asymmetry and the total stiffness of structure affect the final deformation, the final deflection will be reduced if the total stiffness reaches an adequate level although the stiffness asymmetry still exists.

From the above analysis, a high total stiffness of the morphing structure is useful to reduce the rotation under aerodynamic force while a properly selected stiffness asymmetry helps to induce the required rotation angle. The above analysis used the same composite ply angle for both the upper and lower corrugation structure. The unsymmetrical stiffness is only introduced by changing the number of plies in the lower part while a larger stiffness asymmetry can be achieved by using different ply angles. For instance, the following analysis shows the rotation angle and required actuation with a more flexible upper composite layup, where the ply angles are all equal to 90 degrees. Of course, this layup will not be practical in real applications, but the extreme stiffness anisotropy is used here to demonstrate the effect of changing the layup.

Figure 3.10 shows that the rotation angle can be obtained when the stiffness asymmetry exists at the beginning of the calculation. Although the more flexible upper part leads to the larger rotation angle under aerodynamic force, the higher flexibility also provides an even larger rotation angle under the actuation force, 
which fortunately increases the final rotation angle or reduces the required actuation force for the same target angle. The results demonstrate the significant influence of the stiffness asymmetry, which might lead to stronger capabilities of carrying loads and changing shapes.
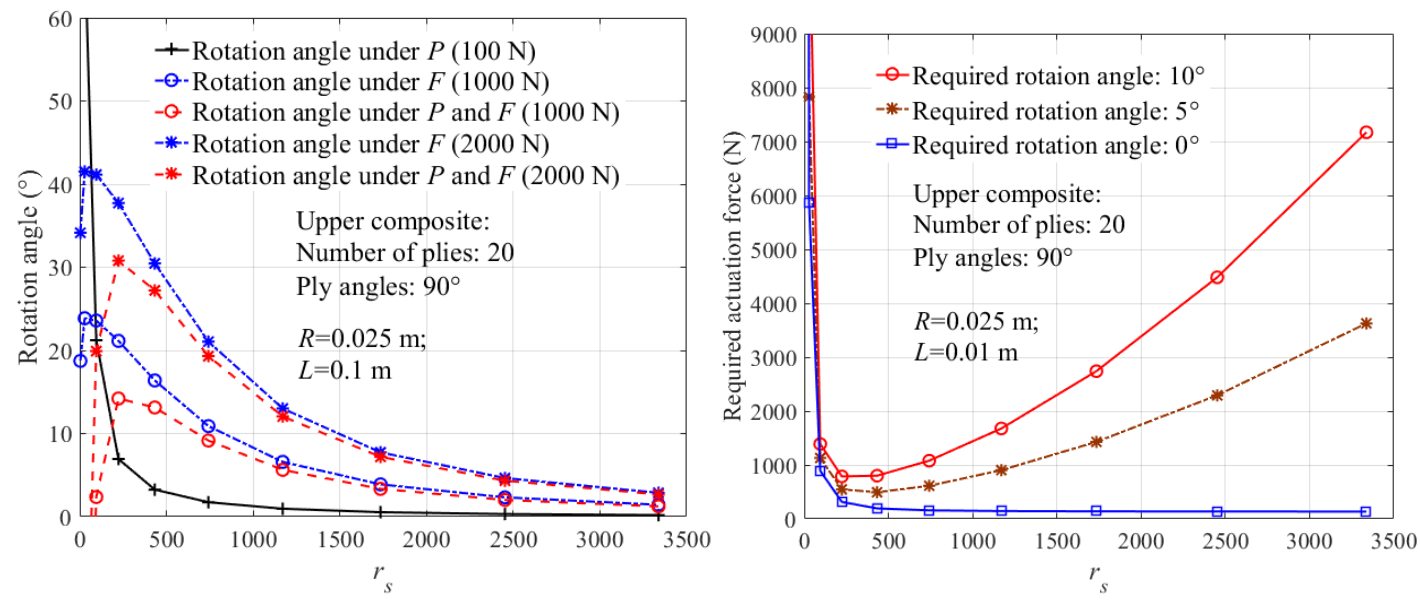

Figure 3.10 (a) Rotation angle and (b) required force with larger stiffness asymmetry

Another aspect of a morphing structure is its deformation limit. The proposed compliant structure needs to ensure its deformation limit has not been reached when it is actuated. The deformation limit criterion determinates the capability of deformation in the morphing direction, which generally can be expressed as the largest strain the morphing structure can undergo before the structure fails. For the composite layup adopted in this section, the axial deformation limit of the corrugated structure is obtained through the Tsai-Wu criterion [112]. The corrugated structure will be regarded as failed when one layer of the composite starts to fail. The detailed finite element model is built in Abaqus ${ }^{\circledR}$, and a user subroutine is applied to monitor the stress level and stops the analysis automatically if the stress level exceeds the Tsai-Wu failure envelope. Once the analysis stops, the subroutine will read the current axial displacement, which is then transferred to the axial deformation limit.

Figure 3.11 shows the deformation limit as a function of the radius $R$ and length $L$ as the number of composite plies changes. Although the composite corrugated panels might not be used in practical cases, the process to satisfy the constraint of the deformation limits represents the essential element in the development, which could 
be replaced by another approach, but should not be neglected.

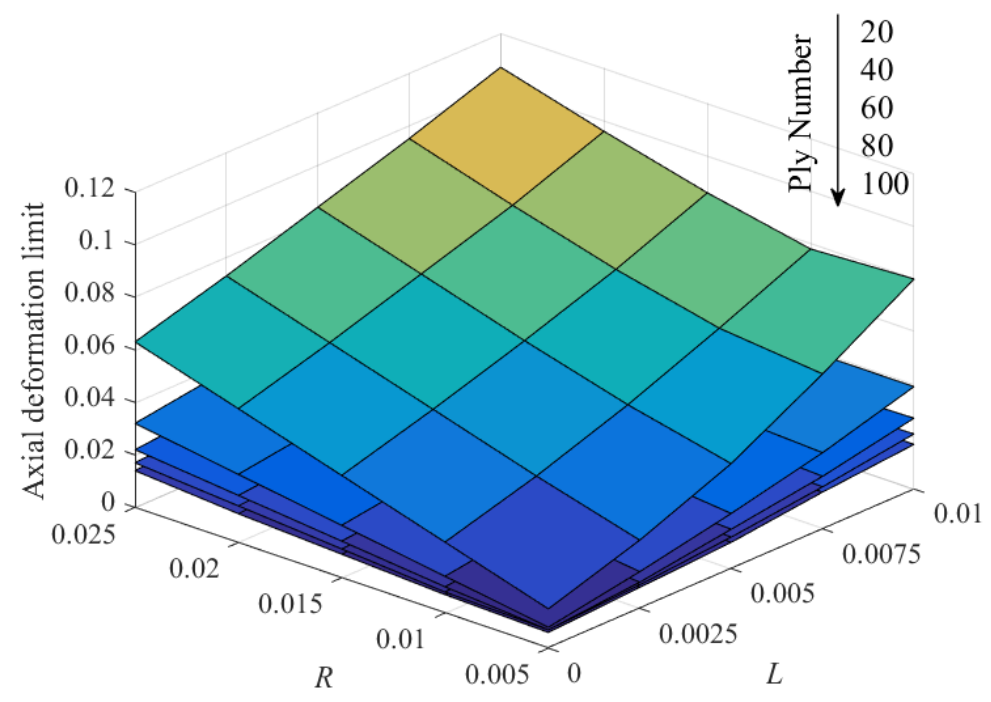

Figure 3.11 Deformation limit of the round corrugation

The simplified model of the proposed compliant structure determines its deformation limit by comparing the axial strain of its upper and lower parts to their corresponding deformation limits. If the axial strains are under their deformation limits, we will assume the structure does not fail.

Figure 3.12 shows the axial strains compared to their corresponding deformation limits under forces $F=1000 \mathrm{~N}$ and $P=100 \mathrm{~N}$. The composite uses the same material data and ply angle as that in Section 3.1. If the deformation is smaller than its limit the corrugation structure is less likely to fail and vice versa. According to the figure, the structural failure is more likely to occur in the upper part since the upper part is more flexible and has a larger axial deformation than the lower part. After the stiffness asymmetry is introduced, the lower composite will have a smaller strain than its limit although the deformation limit in the lower composite is also reduced when the number of its plies increases. 

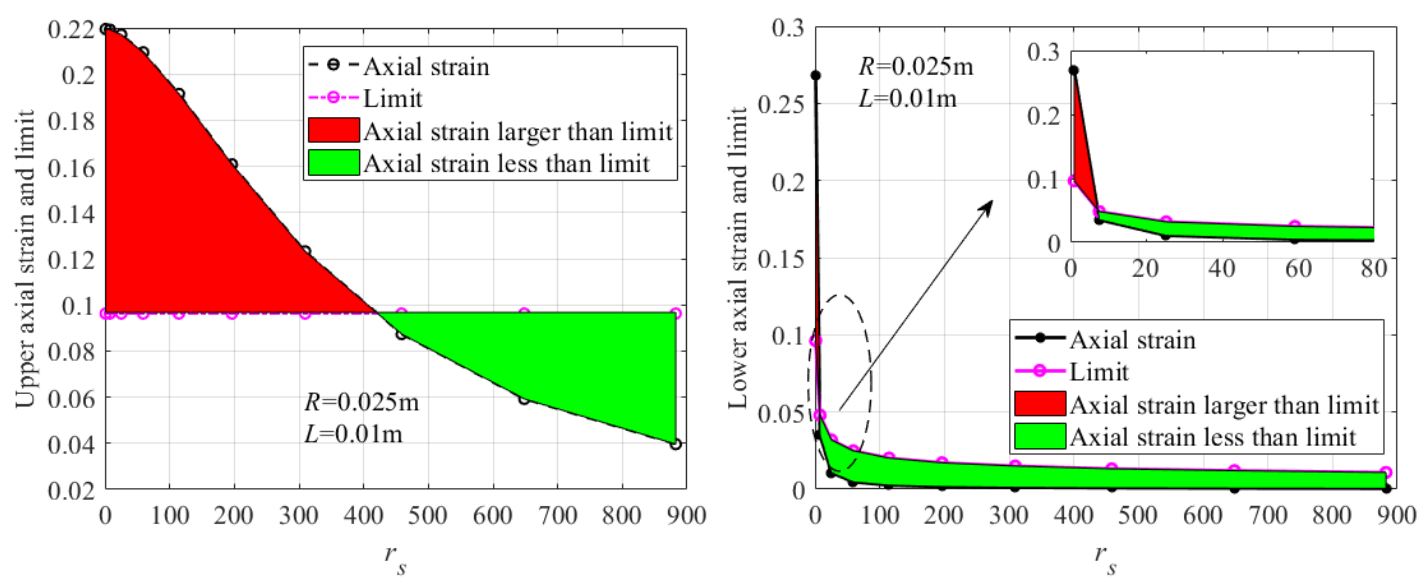

Figure 3.12 Axial strain compared to its limit (a): Upper part, (b): Lower part

\subsection{System level benefit demonstrated in a case study}

\subsubsection{Baseline design}

A baseline design is generated to analyse the fundamental performance after retrofitting the morphing winglet. Since the thesis is focused on the morphing structure, the baseline design is based on the conceptual-level sizing methods from Raymer' book [104]. A twin turboprop airliner is designed as shown in Figure 3.13.

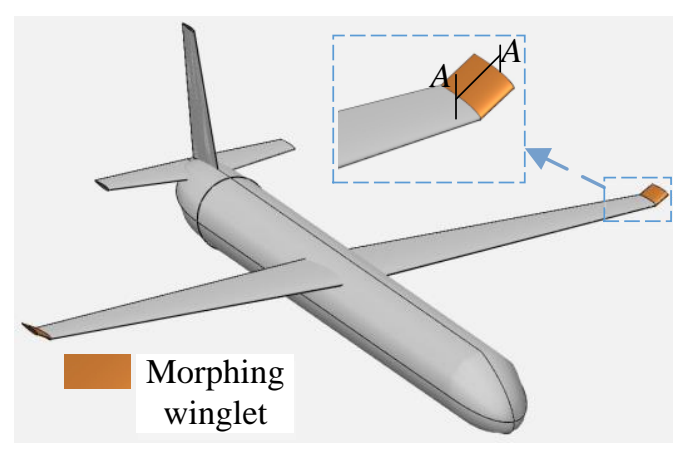

Morphing winglet composed of $n$ units $(n=1,2 .$.

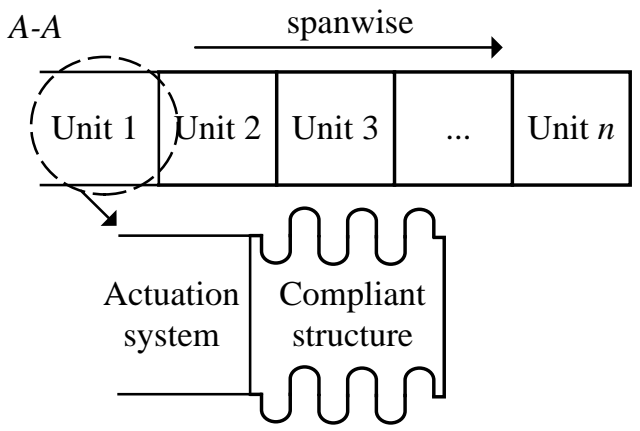

Figure 3.13 A baseline design with the morphing winglet installed

The conceptual sizing estimates the basic parameters such as the geometrical parameters and weight as summarised in Table 3.3. The airfoil is selected from open source data [102] according to the airliner's type. 


\begin{tabular}{|c|c|c|c|}
\hline Number of seats & 70 & Wing Span $(\mathrm{m})$ & 25.38 \\
\hline Range $(\mathrm{km})$ & 1500 & Wing root chord $(\mathrm{m})$ & 3.63 \\
\hline Cruise altitude $(\mathrm{m})$ & 4572 & Leading edge sweep angle $\left(^{\circ}\right)$ & 5 \\
\hline Cruise Mach number & 0.4 & Aspect ratio & 10 \\
\hline Take-off gross weight $(\mathrm{kg})$ & 27217 & Taper ratio & 0.4 \\
\hline Empty weight $(\mathrm{kg})$ & 12063 & Wing tip chord $(\mathrm{m})$ & 1.45 \\
\hline
\end{tabular}

Table 3.3 Baseline design parameters

Figure 3.13 also shows a concept of the morphing winglet retrofitted to the baseline design. The proposed morphing winglet has the same chord and airfoil as the wing tip. No sweep angle of the winglet is applied due to the low baseline wing sweep angle and structure simplification. Multiple units of the proposed compliant structure are used to obtain a larger induced deformation. Since the shape change is due to the elastic deformation of the structure rather than rigid rotation, the winglet will be curved during shape change. More complicated wing tip shapes can also be generated when different deformation is required for the multiple units. While the deformation can be accumulated, the required actuation force will also be increased due to the reaction force of the following units. As shown in Figure 3.13 each unit will be composed of two basic parts: the actuation system and the proposed compliant structure. The actuation system will be described later. The upper and lower surfaces of the compliant structure will be the round corrugated panel.

To provide an adequate deformation limit and reduce the actuation force, the upper ply angle is 90 degrees, and the lower ply angle is 0 or $90^{\circ}$ in turn. Since the upper surface has a larger deformation, its deformation limit needs to be large enough, and the layup of all $90^{\circ}$ in the upper surface ensures the deformation limit is sufficient. The geometry of the round corrugation is $R=0.025 \mathrm{~m}$ and $L=0.01 \mathrm{~m}$, which ensures the corrugated panel has a large deformation limit as shown in Figure 3.11. The structure covers the whole chord from the leading edge to the trailing edge, except for some small gaps between the upper and lower surfaces to avoid a collision. Those gaps might be filled with soft elastomer whose stiffness can be neglected 
compared to the structure. Also, the elastomer will fill in the corrugation structure as the skin of the winglet. According to Equation (3.18), the capability of carrying aerodynamic loads of the proposed compliant structure comes from the actuation force and the stiffness of the structure. Thus, if the elastomer is flexible enough the previous $2 \mathrm{D}$ model is useful to calculate the deflection although a method to prevent wrinkling of the soft elastomer is still needed. The width of the compliant structure is $1.45 \mathrm{~m}$. The morphing winglet is assumed only to change its dihedral angle, and thus no twisting is taken into account. Considering the airfoil thickness and space for the actuation system, the height of the compliant structure is equal to $10 \%$ of the wing tip chord. Although the constant height does not fully represent the airfoil thickness, it provides a straightforward approach to design the morphing structure if only the change of dihedral angle is expected. The length of one compliant structure is assumed to be $0.1 \mathrm{~m}$. The compliant structure is made of the composite described in Table 3.2, which has 20 plies in the upper panel and the axial stiffness ratio $\left(r_{s}\right)$ should be 1170 .

\subsubsection{Actuation system design for the weight estimation}

As analysed above, the actuation force helps to carry the aerodynamic loads. Thus a proper actuation system is essential to the application of the proposed morphing structure. One simple approach is to use a linear actuator which is pinned at the middle between point $\mathrm{A}$ and $\mathrm{D}$ but perpendicular to $\mathrm{BC}$ at point $\mathrm{E}$. Another solution could be the use of an air muscle since the air muscle is flexible and can rotate with the structure. Considering the energy cost and stability, it is also necessary for the actuation system to be self-locking.

In this study, an actuation system is proposed based on a worm and rack mechanism. The proposed mechanism provides a solution for the actuation system and more importantly provides a criterion to estimate the weight of the actuation system. Figure 3.14(a) shows the schematic of the actuation mechanism. One basic set of the actuation mechanism consists of a worm, a rack and accessories, and multiple sets of the actuation mechanism could be used to represent the change of the actuation force 
and weight. The worm is installed in a fixed section and will be actuated by motors to drive the separated rack teeth to slide along the supporting rod LM. Different to a conventional rack, the rack here is separated into single teeth, which enables the teeth to rotate with the morphing structure. Then the rack teeth will slide over the point $\mathrm{M}$ and move to the surface of the internal actuation rod, which is pinned at point $\mathrm{M}$. The internal actuation rod is surrounded by the external actuation rod, along which it can slide. In the figure, the compliant structure is represented by the point A, $\mathrm{B}, \mathrm{C}$ and $\mathrm{D}$. Once the rack starts to be in contact with the external rod, beam BC will be actuated since it is connected to the external rod.

The proposed mechanism is able to provide an actuation force to beam $\mathrm{BC}$, and the self-locking feature of the worm can help to save actuation energy. However, the difficulty of the mechanism is the smooth connection between the separated rack teeth and the internal actuation rod. A convey belt might be used to help the transfer over point M. Also, some accessories are necessary to guarantee the stability of the structure.
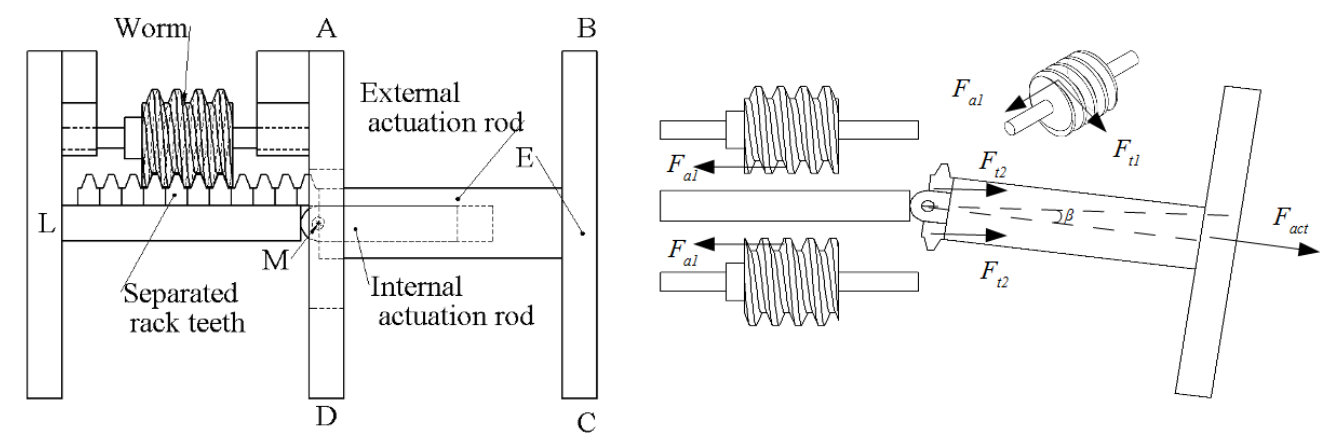

Figure 3.14 (a) Scheme of proposed actuation system, (b) Forces on the mechanism with two worms

Two pairs of worm and rack drive can be used in one set as shown in Figure 3.14(b), which can double the actuation force. The relationship between the actuation force and the rated torque can be derived as [113] 


$$
\begin{aligned}
& 2 F_{t 2} \cos \beta=F_{a c t} \\
& F_{t 2}=F_{a 1} \\
& F_{t 1}=F_{a 1} \tan \left(\gamma_{m}+\rho^{r}\right) \\
& T_{1}=F_{t 1} \frac{1}{2} d_{m 1} \\
& T_{r}=f_{\text {safety }} * T_{1}
\end{aligned}
$$

where $\beta$ is the rotation angle measured from the horizontal line and $F_{a c t}$ is the actuation force on the beam BC. $F_{a 1}$ is the axial force of the worm, and $F_{t 2}$ is the peripheral force on the rack, which is the reaction force of $F_{a l} . F_{t l}$ is the peripheral force on the worm. The lead angle of the worm $\gamma_{m}$ and the reduced friction angle $\rho^{\mathrm{r}}$ can be used to find $F_{t l}$ based on $F_{a l}$. Then the required torque on the worm $T_{l}$ can be obtained from the reference diameter of the worm $d_{m l}$, after which the rated torque $T_{r}$ is obtained by including the safety factor $f_{\text {safety }}\left(f_{\text {safety }}=1.5\right)$.

The maximum actuation force that the mechanism can provide is determined by the maximum loading the worm and rack can bear before failure. The current study determines the strength by considering the tooth root load capacity of the rack, and coefficients obtained in [113] are used. Considering the geometry of the compliant structure, the maximum standard module of the worm is $3 \mathrm{~mm}$ with the actuation force equal to $6335.5 \mathrm{~N}$ and the rated torque for the motor is $15.3 \mathrm{Nm}$. More sets of the proposed actuation system can be used, which changes the required actuation force. Although this method will make the maximum actuation force discrete, it is reasonable to provide the boundaries of the actuation force and weight of the actuation system. Taking the geometry constraint into account, the largest number of actuation mechanisms is 16 for the baseline design.

\subsubsection{Lift to drag ratio and weight estimation}

The proposed application of the compliant structure provides a potential structure solution for the morphing winglet. The morphing winglet could be used to increase span during cruise while remain folded during take-off, landing and taxiing. However, whatever advantages the morphing winglet could bring, one of the side effects is the weight increase due to the installed winglet $\Delta W_{\mathrm{s}}$ and actuation system 
$\Delta W_{\mathrm{a}}$. The range of the aircraft can be calculated using Equation (2.4).

According to this equation, the benefit of a retrofitted morphing winglet, i.e. increased lift to drag ratio, could be reduced by the increased weight.

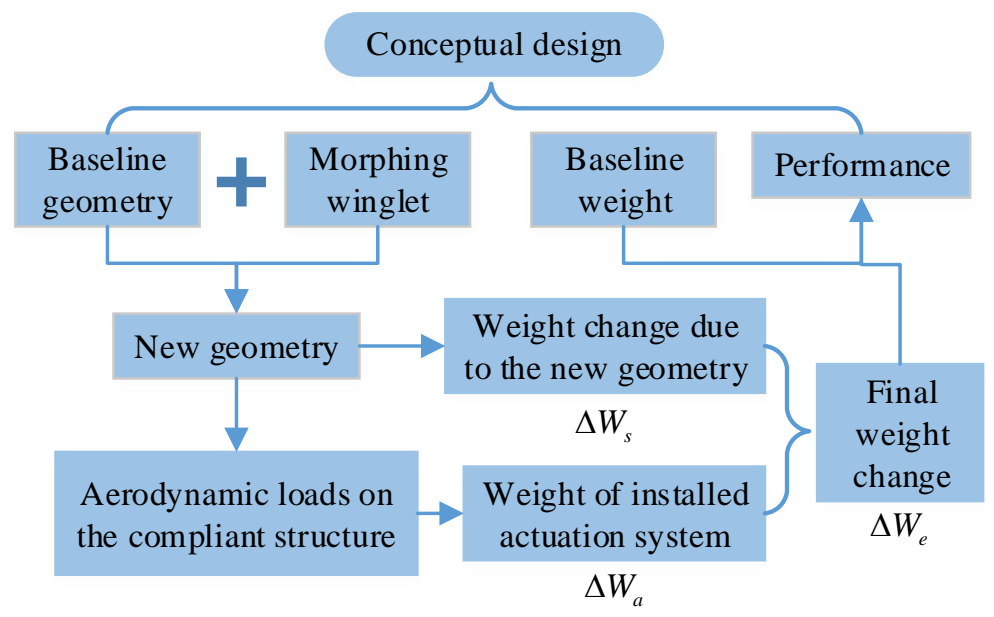

Figure 3.15 Estimation of weight change

As shown in Figure 3.15, the weight change due to the installed winglet is calculated by estimating the weight of the new wing geometry since no existing weight data of the unsymmetrical stiffness structure exists. The new wing geometry is defined with winglet dihedral angle equal to zero, and its weight is estimated using the following equation [104]:

$$
W_{\text {wing }}=0.0051\left(W_{d g} N_{z}\right)^{0.557} S_{w}^{0.649} A_{w}^{0.5}(t / c)_{\text {root }}^{-0.4}(1+\lambda)^{0.1}(\cos \Lambda)^{-1.0} S_{c s w}^{0.1}
$$

where $W_{d g}$ is the gross design weight of the baseline model. $N_{z}$ is the ultimate load factor, which is 1.5 times of the design load factor, and the design load factor is taken as 3.5 in this chapter. $S_{w}$ and $A_{w}$ are the wing area and aspect ratio, which include both the wing of the baseline design and the retrofitted winglet. $(t / c)_{\text {root }}$ is the airfoil thickness ratio at the wing root. $\Lambda$ is the sweep angle at $25 \%$ of the mean aerodynamic chord. $\lambda$ is the wing taper ratio and $S_{c s w}$ is the control surface area of the wing. All the variables in this equation should be converted to Imperial units before use in this equation.

The weight increase of the actuation system is calculated according to the required 
actuation force, which is determined by the aerodynamic force on the winglet. The open software AVL [103] is used for the aerodynamic calculation. AVL is linked with Matlab® for modelling and reading outputs. The forces on each element of the winglet are obtained from the output files and summed to get the aerodynamic force on each unit.

Then the required actuation force of each unit is obtained using the method in Section 3.3.2. According to maximum required actuation force of each unit, the number of proposed actuation sets is determined, which is used to estimate the weight of the worm rack mechanism. For the weight of motors, the required actuation force is transferred to the required rated torque. While the weight of motors varies with their type and application scenarios, a commercially available servo motor database [114] is applied for the estimation and the weight of a motor per Newton-Meter is assumed to be $0.093 \mathrm{~kg}$.

The lift to drag ratio is also calculated by AVL. A trade-off study is then conducted to show the change of both lift to drag ratio and weight with respect to the number of units of the compliant structure retrofitted.

To represent the change in lift to drag ratio, the reference area is the wing area of the baseline design. From the perspective of the potential applications, the flight condition investigated is when the aircraft just takes off and starts to cruise. The lift to drag ratio is calculated with the dihedral angle of the winglet equal to zero, and the required actuation force is determined when the required angle of each unit is 6 degrees. The weight of the fuel remains the same although the increase of lift to drag ratio could reduce the amount of fuel used. Only the weight change due to the retrofitted winglet and actuation is taken into account.

Figure 3.16(a) shows the change in lift to drag ratio and gross weight compared to the baseline design. The number of units retrofitted is from 1 to 15 , which makes the longest winglet cover $11 \%$ of half span. With the increase of the number of units, both lift to drag ratio and weight will increase, while the difference between them will increase until the number of units reaches 10 , after which the weight increase 
will be faster than the lift to drag ratio increase, indicating an adverse effect of the winglet if too many units are installed. The figure could be explained by the increasing required actuation force for each unit, especially the units which are close to the baseline wing tip due to the reaction force of the outboard units. However if the retrofitted winglet is too short, for example when the number of units is equal to 1, the difference between lift to drag ratio and gross weight will also be tiny since the effect of the winglet will be negligible compared to the baseline design.

Figure 3.16(b) shows the required number of actuation sets in each unit with the change of the number of units retrofitted, which represents the required actuation force of each unit. The unit number on the $x$-axis indicates the location of the winglet, and the direction of the arrow shows the increase of units retrofitted. We can find that after the $8^{\text {th }}$ line in the direction of the arrow, the required number of actuation sets on each unit will not be linear regarding the winglet unit number, suggesting the increased required actuation force. According to the geometry constraints, no more than eight units can be used. Thus, eight units of compliant structures should be retrofitted considering both the performance and geometry constraint. In this case, the lift to drag ratio is increased by about $5 \%$ and the weight by about $3.5 \%$, which leads to an increased flight range of about $1.34 \%$ according to Equation (2.4). The deformation of each unit is also within its corresponding limit verified by the method in Section 3.2.
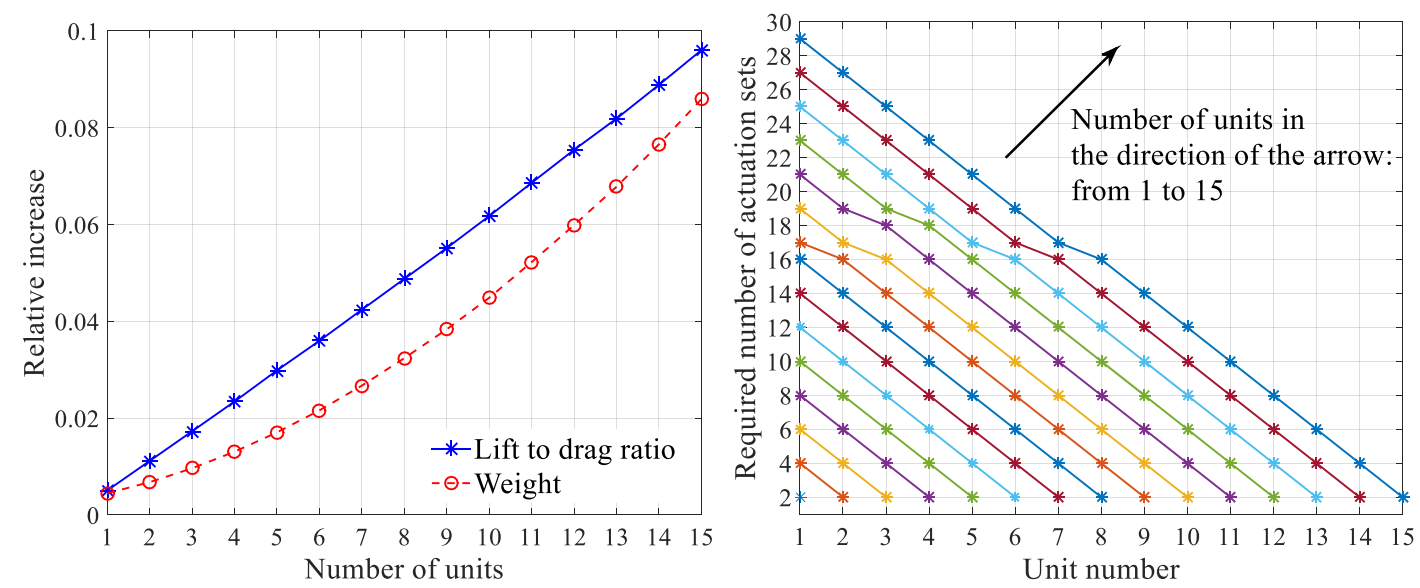

Figure 3.16 (a) Relative increase of lift to drag ratio and weight, (b) Required number of actuation sets 


\subsection{Numerical simulation of a large wing model}

Although the system level study has shown the potential benefits of the morphing winglet, a large wing structure model is built using the baseline wing data from Chapter 2 due to its large scale and challenging flight conditions. The main objective of this model is to test the morphing concept at the conceptual level and find the difficulties of the structure design using the ambitious flight conditions.

The non-morphing part of the wing (baseline wing) is modelled, together with the morphing winglet. This structural model should be able to represent the main characteristics of the wing structure and be easily integrated with the compliant structure. A finite element model is created in Abaqus®. A python script is written to build the wing by inputting the main wing structure parameters.

Figure 3.16 shows the structure model of the wing. Only ribs and spars are considered for simplification and the aerodynamic loads are applied directly to the connection of spars and loads as concentrated forces. The concentrated forces are obtained as equivalent nodal forces from those provided from AVL. The forces are multiplied by the safety factor (1.5) and then applied to the structure.

The load case is chosen at the start of the cruise phase when the aircraft has the largest weight and gravity is also included in the loads. Since attention is paid to the compliant structure the conventional structure of the basic wing is not an optimized solution, but it is still able to represent a typical structure. Different sizes and spar locations have been tested to ensure that the stress and displacement of the baseline wing structure are acceptable as a basis for the morphing winglet. To simplify the baseline structure, the wing is modelled with aluminium except for some steel strengthening.

Round corrugated panels are modelled in this simulation. The optimisation of this large-wing model is not the focus. Thus, the stiffness asymmetry is provided by assigning different composite properties to the different regions in the compliant structure. 


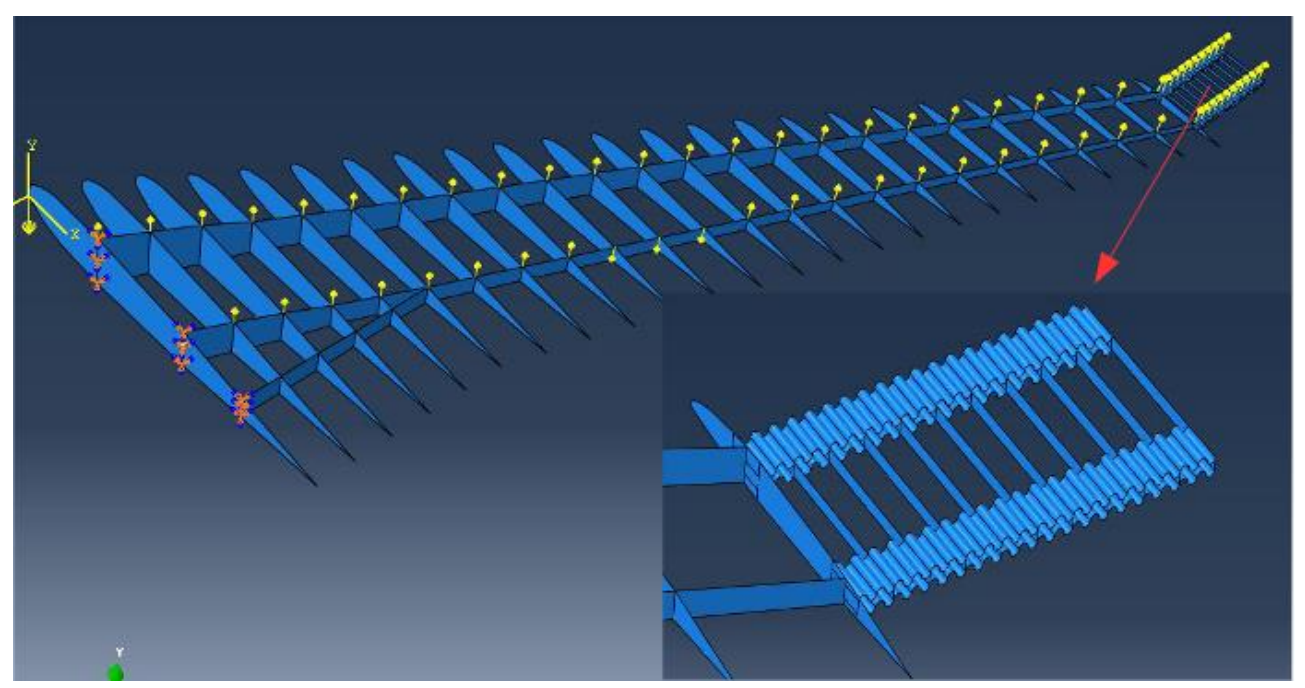

Figure 3.17 Structure and loads of a large wing model

In the previous study, only the winglet dihedral angle is changed and the winglet is modelled without a sweep angle to ease the modelling. The dihedral angle can be changed directly with the rotation of the compliant structure.

A twist angle deformation will also be available if two compliant structures are applied and provide two different rotation angles as shown in Figure 3.16. Two compliant structures based on unsymmetrical stiffness are modelled at the same location of the wing spars. Rib-like structures are modelled to connect the two compliant structures in the leading and trailing edge of the wingtip, which will constrain the shape change caused by the actuation.

The static aeroelastic analysis is conducted by integrating the Abaqus® analysis with AVL in Matlab. The 'restart' method in Abaqus ${ }^{\circledR}$ is used to extend the analysis from the previous step [108] so that the convergence status of the structure can be found. The initial condition is provided when the dihedral angle is 0 . The displacements of the wing structure are then transferred to AVL for the new shape, which generates the updated aerodynamic loads for the next step of the structural analysis. In the analysis, the dihedral and twist angle of both the non-morphing and morphing part of the wing can be updated in the aeroelastic analysis. Figure 3.17 shows the change of the angles and aerodynamic loads over the analysis steps, in which Step 0 corresponds to the initial condition. 
As shown in the figure, the winglet angle and aerodynamic loads converge quickly after three steps, and the final dihedral angle turns out to be around $31^{\circ}$. A twist angle around $6^{\circ}$ can also be found due to the aerodynamic loads on the two compliant structures.
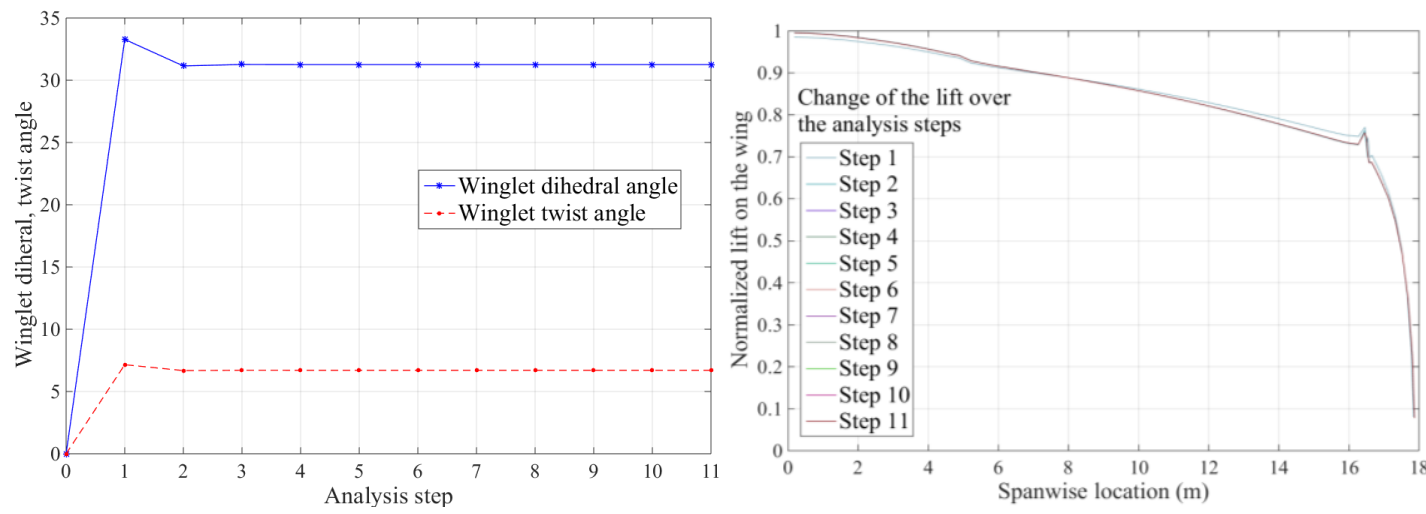

Figure 3.18 (a)Winglet angle, (b) Normalised lift change over the analysis steps

The maximum stress is found at the tip rib of the non-morphing part of the wing, which is connected to the compliant structure. Figure 3.18 shows the deformation and stress distribution.

The displacement under aerodynamic loads is too large to be neglected, and thus the actuation force is required to maintain the specified dihedral angle. In this test, the actuation system of the morphing winglet is modelled as a beam that is able to carry some of the loads. The winglet will be too flexible if the stiffness of the actuation system is neglected. Numerical tests have been performed to determine a suitable size for the beam, which turns out to be a flanged beam made of steel, whose height is half of the compliant structure height.

The simulation results indicate that for the large wing structure in a flight condition similar to an airliner, the requirement of actuation force will be very large, which will result in a significant weight increase. The optimisation of the compliant structure is demanded to reduce the actuation force otherwise it is difficult to apply the morphing concept at the large wing scale with the challenging flight conditions. 


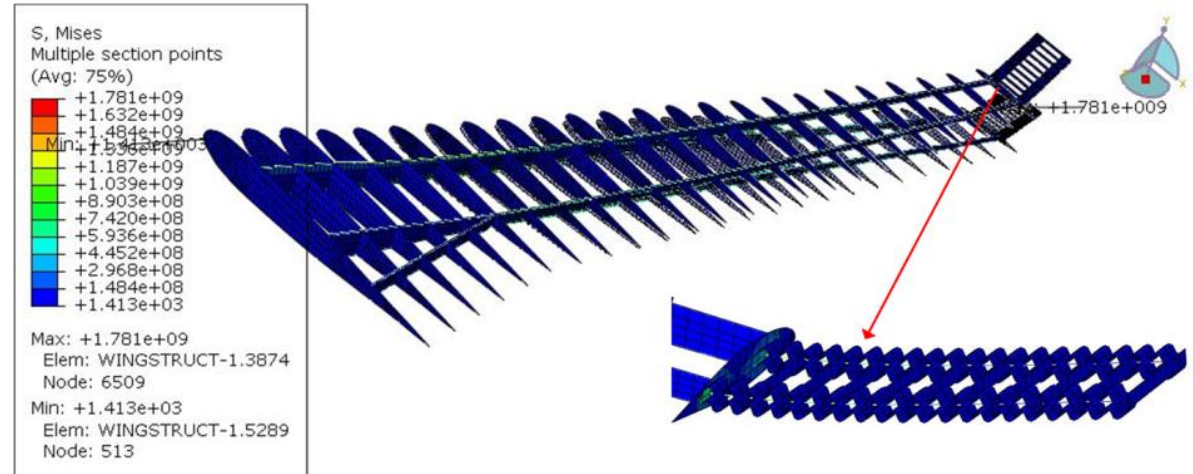

Figure 3.19 Deformation and stress distribution of the wing

\subsection{Summary}

In this chapter, an innovative, compliant structure is proposed making use of the unsymmetrical stiffness of the structure at the conceptual level to find a solution to change the winglet dihedral angle. The compliant structure consists of the upper and lower parts, which are connected by a relatively rigid part. By increasing the stiffness of the lower part, while fixing the stiffness of the upper part, the stiffness asymmetry is increased together with the total stiffness. A simplified 2-dimensional model is built to estimate the deformation of the structure analytically.

From the above analysis, we can conclude that the final deformation of the structure is determined by the total stiffness, stiffness asymmetry, aerodynamic loads and actuation force. By allocating unsymmetrical stiffness into the morphing structure, we can achieve rotation deformation for the structure from linear actuation. Furthermore, while the increasing total stiffness of the structure can help to carry aerodynamic loads, the stiffness asymmetry is also able to induce a large rotation angle in the opposite direction. With adequate total stiffness and properly selected stiffness asymmetry, the morphing structure is able to carry aerodynamic loads and change its shape simultaneously. The morphing winglet can benefit from the unsymmetrical stiffness of the morphing structure. By increasing the total stiffness of the structure and allocating the unsymmetrical stiffness simultaneously, the novel compliant structure helps to reduce the requirement of the actuation system. Since only a linear actuation force is required, a large range of actuation systems can be 
applied including a conventional actuator, which provides more choices and more reliability.

With the help of this simplified model, the application of the morphing structure is investigated showing the potential pros and cons. A baseline design is generated to provide the necessary information for comparison. A basic actuation mechanism, which can represent the essential elements of a self-locking mechanism, is also introduced to provide the solution for constant actuation as well as weight estimation. The change of lift to drag ratio and weight are analysed with different numbers of units retrofitted to the baseline design. It is found after a critical point the increase of weight will be faster than the increase of lift to drag ratio. Also, the geometry of the baseline design constrains the number of actuation sets. Thus, a trade-off selection should be made according to the situation. Eight units of morphing structure are used, increasing the lift to drag ratio by about $5 \%$ and the weight by about $3.5 \%$.

It should be noted that in this chapter, the different stiffnesses are provided by changing the number of the composite plies of the round corrugations. While this could lead to some unpractical composite layups, this chapter intends to introduce the novel concept of the compliant structure at the conceptual level. The critical feature of the proposed compliant structure is the stiffness asymmetry and this chapter used the round corrugated made of composite layups as a reference to provide the different axial stiffnesses. In real-world applications, the stiffness asymmetry can be provided by changing the geometry or sizes of the corrugated panels as presented in Chapter 5 and 6. Other forms such as isotropic or anisotropic plates can be used, as used in the verification examples in Figure 3.6.

Although the thesis demonstrates the application of the compliant structure to morphing aircraft, the proposed compliant structure can also be applied in other industrial fields, such as robotics. Since its deformation is induced by the actuation force, rather than temperature, it is easier to control the final deformation compared to its thermal analogy. Also, the response speed of the structure under actuation can be faster, and the structure can be less sensitive to the change of the environment 
temperature.

Numerical simulation of a large wing structure has also been performed. The simulation indicates the structure should be optimised if it is expected to be used in the wing structure similar to an airliner with its flight conditions.

This conceptual study has shown the analytical expressions and basic properties of the proposed compliant structure. The investigation also indicates the requirements of an accurate model of the corrugated panel, since the optimisation of the compliant structure needs to predict the deformation with more practical geometry parameters and material properties. The equivalent model of the corrugated panels and compliant structures will be developed in Chapters 4 and 5, and the optimisation will be performed in Chapter 5 and 6. 


\section{Chapter 4 Equivalent model of corrugated panels}

\subsection{Background and model definition}

The corrugated structure is intensively investigated in the field of morphing aircraft due to its capability of high anisotropy and more substantial deformation. Although detailed finite element analysis can be used to simulate its mechanical responses, an efficient equivalent model of the corrugated panel is essential for its structural analysis and optimisation.

Yokozeki et al. [31] developed the analytical solution of the equivalent axial and transverse tensile and flexural moduli with the round corrugation. The analytical solution provided a reasonable accuracy compared to the experimental results. Samanta and Mukhopadhyay [115] derived analytical solutions of the axial and transverse equivalent moduli of the trapezoidal corrugated panel. The experimental investigation of Thill et al. [32] showed a three-stage stress-strain relationship of the trapezoidal corrugated panel, in which the first linear stage had a relatively good agreement with the analytical solution modified from [115]. Xia et al. [111] developed a complete approach of the corrugated panel, which gives the equivalent model of round and trapezoidal corrugations. The method provides the closed forms of the six effective components in the stiffness matrix of the equivalent orthotropic plate. Comparisons to other analytical results and the finite element method have shown the accuracy of the equivalent model. In this method, the equivalent orthotropic plate is a classical Kirchhoff plate, which does not consider the transverse shear. A recent publication from Mohammadi et al. [116] derived the transverse shear modulus, which few researchers have studied. The equivalent models of corrugated cores with elastomeric coatings were also derived by Dayyani 
et al. [110].

Although the equivalent properties of the corrugated panel have been studied extensively, this chapter investigates another aspect, namely the axial and bending coupling when the corrugated panel has a fixed boundary condition, to which little attention has been paid in the literature. The corrugated panel will have a vertical deflection caused by the pure extension load when it has a fixed boundary condition. If the corrugated panel has a pinned boundary condition, no vertical deflection will occur. This deflection-extension coupling effect could be negligible in some cases. However, it can also have a significant influence on the structure deformation, especially for the morphing applications where the corrugated structures are often required to have a relatively large deformation. For example, if the corrugated structure is used as the morphing skin, the tendency of the out-of-plane deflection could change the wing shape or increase the actuation energy. The current investigation can also provide guidelines for installing the corrugated panels in real-world applications. In the previous references, the symmetry of the structure is usually applied and only half [115] or quarter [31, 116] of the corrugation unit is analysed. The fixed boundary condition is applied to calculate the strain energy and internal moment. For the homogeneous method [111] the boundary condition will not affect the equivalent properties of the entire structure since the boundary conditions are constrained when calculating the strain energy.

With the fixed boundary condition, vertical deflections under pure extension load will be analysed together with axial deflections. The axial deflections are applied to calculate the equivalent axial moduli, which are compared to those from the existing models. The coupling effect can be represented as an effect of the boundary condition if the entire corrugated panel is separated into two segments. As a supplement to the previous equivalent model, a modification to the equivalent model proposed in the reference [111] is made by introducing a coupling component.

A more general method can be used to model the corrugated panel if deflections of the equivalent model can be represented by its stiffness matrix. Coupling terms 
between the axial force and vertical deflection and rotation angle are found analytically. After representing this deflection, the influence of considering this coupling effect is shown on the compliant structure based on unsymmetrical stiffness. The method to eliminate this coupling is also proposed by using an offset boundary condition.
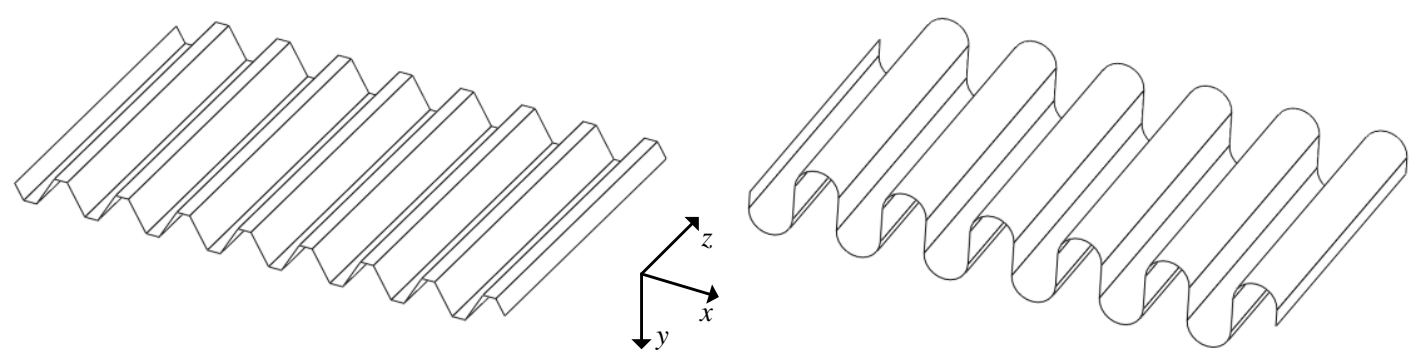

Figure 4.1 (a) Trapezoidal corrugation panel, (b) Round corrugation panel

Figure 4.1 shows the shape of the trapezoidal and round corrugation panels, which are the subject of this study. As a periodic structure in the $x y$ plane, the entire shape and size of a corrugated structure are determined by the shape of a single unit and the number of corrugation units.

In the $2 \mathrm{D}$ case, the unit of the trapezoidal corrugated panel consists of the straight beams $\mathrm{AB}, \mathrm{BC}, \mathrm{CD}, \mathrm{DE}, \mathrm{EF}$ and $\mathrm{FG}$, and the round unit is made of straight beams $\mathrm{AB}, \mathrm{CD}, \mathrm{EF}$ and curved beams BC, DE, as shown in Figure 4.2.

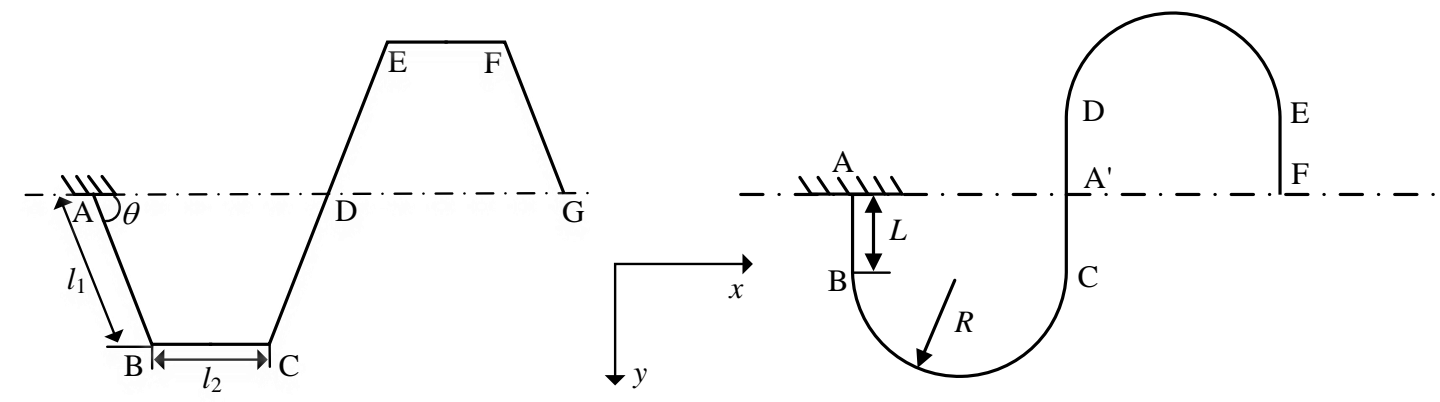

(a)

(b)

Figure 4.2 Geometry of the corrugation unit: (a) trapezoidal profile, (b) round profile The geometry variables of the trapezoidal corrugation are denoted by the inclined length $l_{1}$, horizontal length $l_{2}$, and inclined angle $\theta$. The geometry variables of the round corrugation are denoted by the straight part $L$, and radius $R$. The corrugation 
unit is repeated in the $x$-direction, and the number of corrugation units is denoted by the variable $n$. The thickness of the corrugated panel is denoted by the variable $t$.

The corrugated panel is supposed to be clamped at the point $\mathrm{A}$ as shown in the figure, which simulates a fixed boundary condition.

\subsection{Deflections caused by axial loads}

\subsubsection{Assumptions and method to calculate the deflections}

The deflections caused by the axial load will be firstly investigated in this section since the corrugated panel is mainly actuated in the $x$-direction in morphing aircraft and the method used here can also be applied to other load cases.

The deflections of the corrugated panels are calculated following the assumptions as

1) The separate beams consisting of the corrugated panel are rigid-connected;

2) The deformation of each beam is not influenced by the other beams;

3) The deflections of the points in the corrugated panel can be accumulated.

The deflection of each separate beam can be calculated using classical mechanics and Castigliano's second theorem if the internal load is known under specific external loads. The deflection of the unit corrugation, which is represented by the deflections of point $\mathrm{G}$ (trapezoidal corrugation) or $\mathrm{F}$ (round corrugation) is then calculated by accumulating the local deflections of the beams and considering the rigid translation caused by the rotation angles of the beam cross-sections. Figure 4.3 shows the schematic of the deflection components.
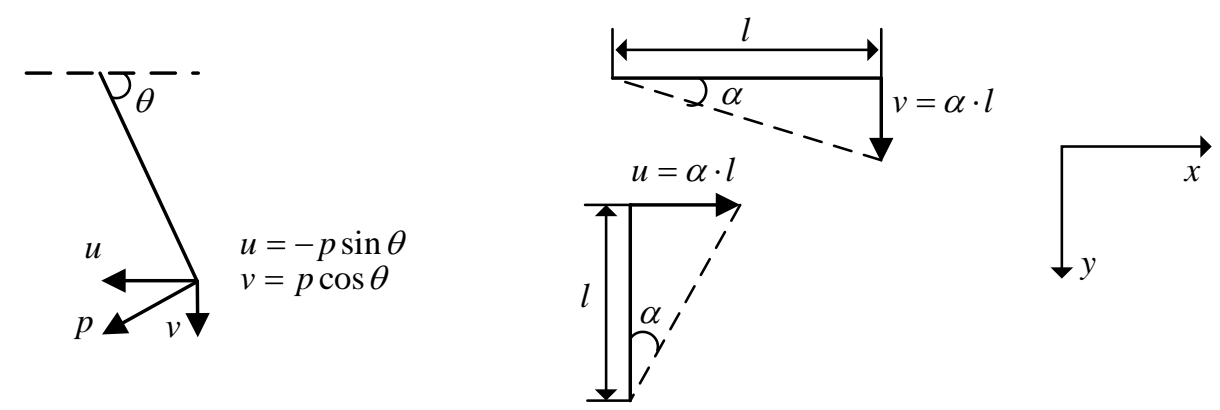

Figure 4.3 Schematic of the deflection components 
For a beam with the inclined angle $\theta$, the deflection of the beam $p$, which is perpendicular to the beam, can be decomposed to the axial deflection $u$ and the vertical deflection $v$. According to the inclined angle and direction definition, we have $u=-p \sin \theta$, and $v=p \cos \theta$.

Also, the rotation angle of the beam cross-section will lead to a rigid translation. The rotation angle $\alpha$ of an axial beam will cause a vertical deflection $v$ and an axial deflection $u$ can be obtained if the rotation angle occurs in a vertical beam.

\subsubsection{Deflections calculation}

The axial and vertical deflections of the trapezoidal and round corrugation unit are both calculated in this section.

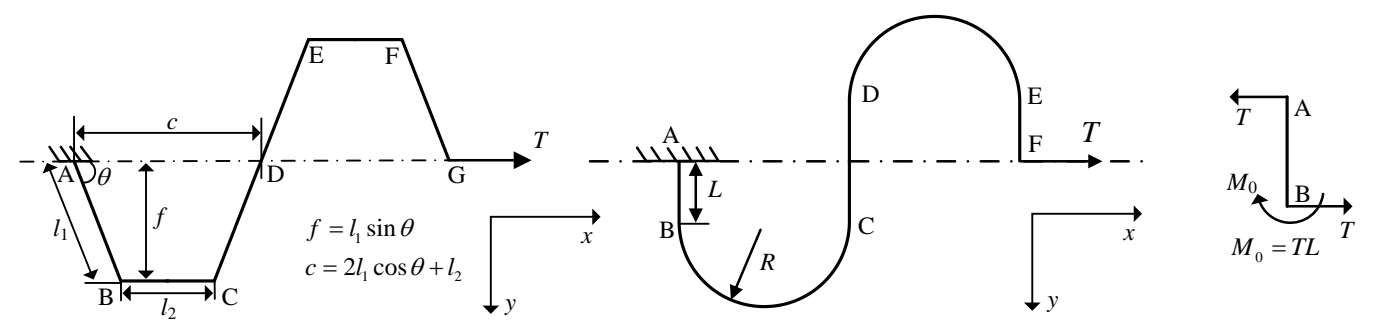

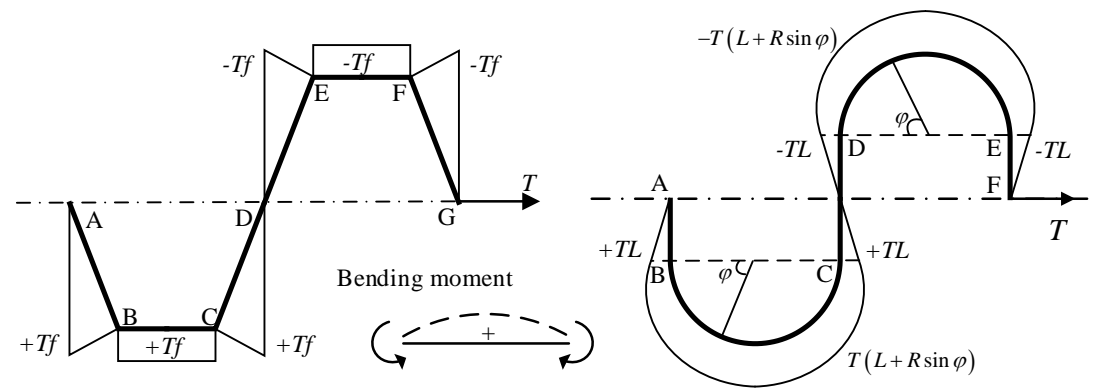

(a) (b)

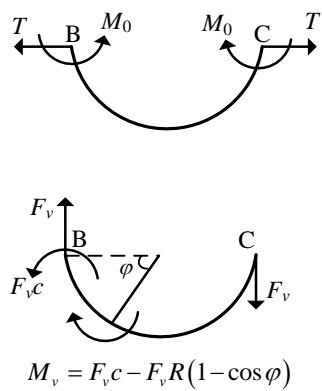

(c)

Figure 4.4 Geometry and bending moment in the corrugation unit: (a): trapezoidal, (b): round, (c): separate beams in the round unit

Figure 4.4 (a) shows the profile of the trapezoidal corrugated panel and internal load caused by the axial load $T$ in the $x$-direction. Based on the assumptions, the beams are rigidly connected to the previous part, and thus the global deflection of a point depends on the deformation of the previous beams. For example, the vertical deflection of point $\mathrm{C}$ includes three parts: the global vertical deflection of point $\mathrm{B}$, the local vertical deflection of beam $\mathrm{BC}$ and the rigid translation of point $\mathrm{B}$ caused 
by the rotation angle of the beam cross-section at point $\mathrm{B}$. The rotation angle of the beam cross-section will also be accumulated by the local rotation angles. Then, the global vertical deflection of point $\mathrm{C}, v_{C}$, can be calculated as

$$
v_{C}=v_{B}+v_{B C}+\alpha_{B} \cdot l_{2}
$$

The subscript ' $A B$ ' or ' $B C$ ' means the local deflection, or beam cross-section rotation angle of that beam, the subscript of a single point ' $B$ ' or ' $C$ ' means the global value at that point.

Thus, $v_{B C}$ is the local vertical deflection of beam $\mathrm{BC}$, and $v_{B}$ and $\alpha_{B}$ are the global deflection and rotation angle of the cross-section of point B. Also for local deformation of beams $\mathrm{AB}$ and $\mathrm{BC}$, we have

$$
\begin{gathered}
p_{A B}=\frac{T l_{1}^{3} \sin \theta}{6 E I}, \alpha_{A B}=\frac{T l_{1}^{2} \sin \theta}{2 E I} \\
v_{B}=p_{A B} \cos \theta=\frac{T l_{1}^{3} \sin \theta \cos \theta}{6 E I} \\
u_{B}=-p_{A B} \sin \theta=-\frac{T l_{1}^{3}(\sin \theta)^{2}}{6 E I} \\
p_{B C}=\frac{T l_{1} l_{2}^{2} \sin \theta}{2 E I}, \alpha_{B C}=\frac{T l_{1}^{2} \sin \theta}{2 E I} \\
v_{B C}=p_{B C} \cos 0=\frac{T l_{1} l_{2}^{2} \sin \theta}{2 E I} \\
u_{B C}=p_{B C} \sin 0=0 \\
v_{B}=v_{A B}, u_{B}=u_{A B} \\
\alpha_{B}=\alpha_{A B}, \alpha_{C}=\alpha_{B}+\alpha_{B C}
\end{gathered}
$$

where $E$ is Young's modulus, $I$ is the second moment of area. Repeating the above steps for the other beams, the deflections and rotation angle at point $G$ can be calculated, which are equal to the deflections and rotation angle of the unit as 


$$
\begin{aligned}
& u_{\text {unit }}=u_{G}=\frac{2}{3} \frac{T l_{1}^{2}(\sin \theta)^{2}\left(2 l_{1}+3 l_{2}\right)}{E I} \\
& v_{\text {unit }}=v_{G}=\frac{T l_{1} \sin \theta\left(l_{1}+l_{2}\right)\left(l_{2}+2 l_{1} \cos \theta\right)}{E I} \\
& \alpha_{\text {unit }}=\alpha_{G}=0
\end{aligned}
$$

For the round corrugation, the same approach can be applied to obtain the vertical deflection and rotation angle of a single unit. The geometry and internal bending moment of the round corrugation unit are shown in Figure 4.4(b). The curved beams are assumed to be thin enough compared to the radius $R$, that the beam theory for a straight beam may be applied. The local vertical deflection of the curved beam BC is obtained by the Castigliano's second theorem as

$$
\begin{aligned}
& v_{B C}=\int_{0}^{\pi} \frac{M}{E I} \frac{\partial M}{\partial F_{v}} \cdot R d \varphi \\
& M=T R \sin \varphi+M_{0}+F_{v}[c-R(1-\cos \varphi)]
\end{aligned}
$$

where $M$ is the bending moment in the curved beam $\mathrm{BC}$, and $\varphi$ is the current angle with respect to the $x$-axis. $M_{0}=T L$ and $F_{v}$ is a virtual vertical force applied at point $\mathrm{C}$ to obtain the vertical deflection.

The deflections and rotation angle of a round corrugation unit can be obtained using the same approach to the trapezoidal corrugation as

$$
\begin{aligned}
& u_{\text {unit }}=u_{F}=\frac{1}{3} \frac{T\left(4 L^{3}+6 \pi L^{2} R+24 L R^{2}+3 \pi R^{3}\right)}{E I} \\
& v_{\text {unit }}=v_{F}=\frac{2 T R\left(L^{2}+2 R^{2}+\pi L R\right)}{E I} \\
& \alpha_{\text {unit }}=\alpha_{F}=0
\end{aligned}
$$

From the above equation, we can find the rotation angle of the beam cross-section will be zero again after a whole unit, which means for the next unit the previous unit will not affect its global deflection by the rigid translation. It is caused by the symmetrical internal bending moment in the whole corrugation unit. Thus, the global deflections of $n$ units can be expressed as 


$$
\begin{aligned}
& u_{n}=n \cdot u_{\text {unit }} \\
& v_{n}=n \cdot v_{\text {unit }}
\end{aligned}
$$

The deflections and beam cross-section rotation angle of the different points in the trapezoidal and round corrugation unit are listed in the Tables 4.1, 4.2, and 4.3.

\begin{tabular}{|c|c|c|c|}
\hline \multicolumn{2}{|c|}{ Trapezoidal corrugation unit } & \multicolumn{2}{c|}{ Round corrugation unit } \\
\hline Point & Axial deflections & Point & Axial deflections \\
\hline B & $u_{i} i=A, B, C, \ldots G$ & $u_{i} i=A, B, C, \ldots F$ \\
\hline $\mathrm{C}$ & $-\frac{1}{6} \frac{T l_{1}^{3}(\sin \theta)^{2}}{E I}$ & $\mathrm{~B}$ & $-\frac{1}{6} \frac{T L^{3}}{E I}$ \\
\hline $\mathrm{D}$ & $\frac{1}{3} \frac{T l_{1}^{2}(\sin \theta)^{2}\left(2 l_{1}+3 l_{2}\right)}{E I}$ & $\mathrm{D}$ & $\frac{1}{2} \frac{T\left(3 L^{3}+4 \pi L^{2} R+12 L R^{2}+\pi R^{3}\right)}{E I}$ \\
\hline $\mathrm{E}$ & $\frac{1}{2} \frac{T l_{1}^{2}(\sin \theta)^{2}\left(3 l_{1}+4 l_{2}\right)}{E I}$ & $\mathrm{E}$ & $\frac{1}{2} \frac{T\left(3 L^{3}+4 \pi L^{2} R+16 L R^{2}+2 \pi R^{3}\right)}{E I}$ \\
\hline $\mathrm{F}$ & $\frac{1}{2} \frac{T l_{1}^{2}(\sin \theta)^{2}\left(3 l_{1}+4 l_{2}\right)}{E I}$ & $\mathrm{~F}$ & $\frac{1}{3} \frac{T\left(4 L^{3}+6 \pi L^{2} R+24 L R^{2}+3 \pi R^{3}\right)}{E I}$ \\
\hline $\mathrm{G}$ & $\frac{2}{3} \frac{T l_{1}^{2}(\sin \theta)^{2}\left(2 l_{1}+3 l_{2}\right)}{E I}$ & $\mathrm{E}$ & $\mathrm{EI}$ \\
\hline
\end{tabular}

Table 4.1 Axial deflections of the points in the corrugation unit 


\begin{tabular}{|c|c|c|}
\hline Point & $\begin{array}{c}\text { Rotation angle of the beam } \\
\text { cross section }\end{array}$ & Vertical deflection $v_{i} i=A, B, C, \ldots G$ \\
\hline \multirow{2}{*}{ B } & $\underline{1} \underline{T l_{1}^{2} \sin \theta}$ & $\underline{1} \underline{T l_{1}^{3} \sin \theta \cos \theta}$ \\
\hline & $2 \quad E I$ & $6 \quad E I$ \\
\hline \multirow{2}{*}{$\mathrm{C}$} & $1 T l_{1} \sin \theta\left(l_{1}+2 l_{2}\right)$ & $\underline{1} \underline{T l_{1} \sin \theta\left(l_{1}^{2} \cos \theta+3 l_{1} l_{2}+3 l_{2}^{2}\right)}$ \\
\hline & $E I$ & $E I$ \\
\hline \multirow{2}{*}{ D } & \multirow{2}{*}{$\frac{T l_{1} \sin \theta\left(l_{1}+l_{2}\right)}{E I}$} & $1 T l_{1} \sin \theta\left(2 l_{1}^{2} \cos \theta+2 l_{1} l_{2} \cos \theta+l_{1} l_{2}+l_{2}^{2}\right)$ \\
\hline & & EI \\
\hline \multirow[t]{2}{*}{ E } & $\underline{1} T l_{1} \sin \theta\left(l_{1}+2 l_{2}\right)$ & $1 T l_{1} \sin \theta\left(11 l_{1}^{2} \cos \theta+12 l_{1} l_{2} \cos \theta+3 l_{1} l_{2}+3 l_{2}^{2}\right)$ \\
\hline & 2 & $E I$ \\
\hline \multirow{2}{*}{$\mathrm{F}$} & $1 T l_{1}^{2} \sin \theta$ & ${ }_{1} T l_{1} \sin \theta\left(11 l_{1}^{2} \cos \theta+12 l_{1} l_{2} \cos \theta+6 l_{1} l_{2}+6 l_{2}^{2}\right)$ \\
\hline & $2 \quad E I$ & $E I$ \\
\hline G & 0 & $\frac{T l_{1} \sin \theta\left(l_{1}+l_{2}\right)\left(2 l_{1} \cos \theta+l_{2}\right)}{E I}$ \\
\hline
\end{tabular}

Table 4.2 Rotation of the beam cross-section and vertical deflections in the trapezoidal corrugation unit

In the tables, we can find the beam cross-section rotation angles are symmetrical about the centre line of the corrugation unit, while the axial and vertical deflections are accumulated through the points in the corrugation unit. 


\begin{tabular}{|c|c|c|}
\hline Point & $\begin{array}{l}\text { Rotation angle of the beam cross } \\
\text { section }\end{array}$ & $\begin{array}{l}\text { Vertical deflection } \\
v_{i} i=A, B, C, \ldots F\end{array}$ \\
\hline B & $\frac{1}{2} \frac{T L^{2}}{E I}$ & 0 \\
\hline \multirow{2}{*}{$\mathrm{C}$} & $1 T\left(L^{2}+4 R^{2}+2 \pi L R\right)$ & $T R\left(L^{2}+2 R^{2}+\pi L R\right)$ \\
\hline & $E I$ & $E I$ \\
\hline \multirow{2}{*}{ D } & $1 T\left(L^{2}+4 R^{2}+2 \pi L R\right)$ & $T R\left(L^{2}+2 R^{2}+\pi L R\right)$ \\
\hline & 2 & $E I$ \\
\hline \multirow{2}{*}{$\mathrm{E}$} & $\underline{1} \underline{T L^{2}}$ & $2 T R\left(L^{2}+2 R^{2}+\pi L R\right)$ \\
\hline & $2 E I$ & $E I$ \\
\hline \multirow{2}{*}{$\mathrm{F}$} & \multirow{2}{*}{0} & $2 T R\left(L^{2}+2 R^{2}+\pi L R\right)$ \\
\hline & & $E I$ \\
\hline
\end{tabular}

Table 4.3 Rotation of the beam cross-section and vertical deflections in the round corrugation unit

\subsubsection{Numerical verification and a modified equivalent model}

The axial deflections are used to calculate the equivalent modulus in the axial direction. The equivalent modulus in the axial direction can be expressed for the trapezoidal corrugation as

$$
E_{x}=\frac{T / A}{u_{\text {unit }} /(2 c)}=\frac{3 E I\left(2 l_{1} \cos \theta+l_{2}\right)}{A l_{1}^{2}(\sin \theta)^{2}\left(2 l_{1}+3 l_{2}\right)}
$$

where $A$ is the area of the corrugation cross-section.

For the round corrugation, we have

$$
E_{x}=\frac{12 E I R}{A\left(4 L^{3}+6 \pi L^{2} R+24 L R^{2}+3 \pi R^{3}\right)}
$$

The axial equivalent moduli are then compared to those from existing methods and the finite element method. The finite element models are built in Abaqus ${ }^{\circledR}$ [108]. To ensure accuracy, a mesh convergence study is first performed, which gives a mesh 
size $0.001 \mathrm{~m}$. Both the Euler beam element $B 33$ and the Timoshenko beam element $B 31$ are used for the verification, which are labelled as 'FEM (A)' and 'FEM (B)' in Figures 4.5 and 4.6 respectively. The equivalent methods from $[31,111,115]$ are applied for the comparisons. The corrugated panel is assumed to be made of the Aluminum sheet with a width $0.01 \mathrm{~m}$.
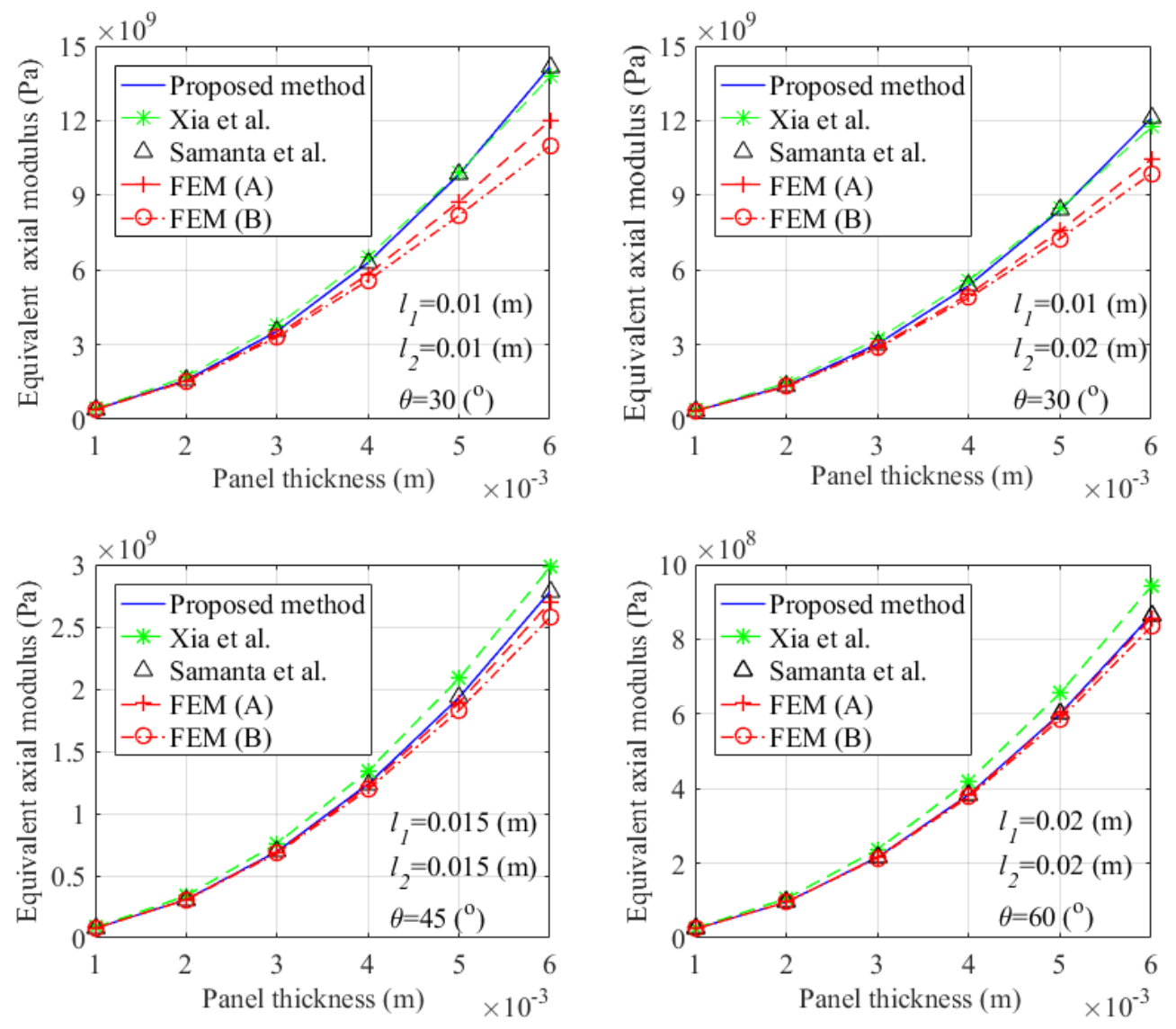

Figure 4.5 Equivalent axial modulus of the trapezoidal corrugation panel

Figure 4.5 shows the equivalent axial modulus of the trapezoidal corrugation panel with different corrugation shapes. The proposed method has results identical to those from [115] since in the reference the equivalent modulus was also obtained by calculating the deflection under extension loads, although only half of the corrugation was considered. The homogenous method has some differences to the proposed method. Compared to the finite element method, all the analytical methods have some errors. The error is relatively large when the plate becomes thick compared to the size of the trapezoid. Also, the Euler beam element has closer 
results to the proposed method since the method uses the classical beam theory and no transverse shear in the beam is considered. If the corrugated panel is supposed to be applied in the field of morphing structures, the beam will be relatively thin since the large thickness of the beams will increase the stress in the structures, which constrains the deformation limit of the morphing structure. The beams with large thicknesses (e.g. $6 \mathrm{~mm}$ ) are impractical since the accuracy of the beam models would be poor and the maximum stresses would be high, but these results are included because the errors are so small for the thicknesses that would be used in practice (e.g. less than $2 \mathrm{~mm})$.
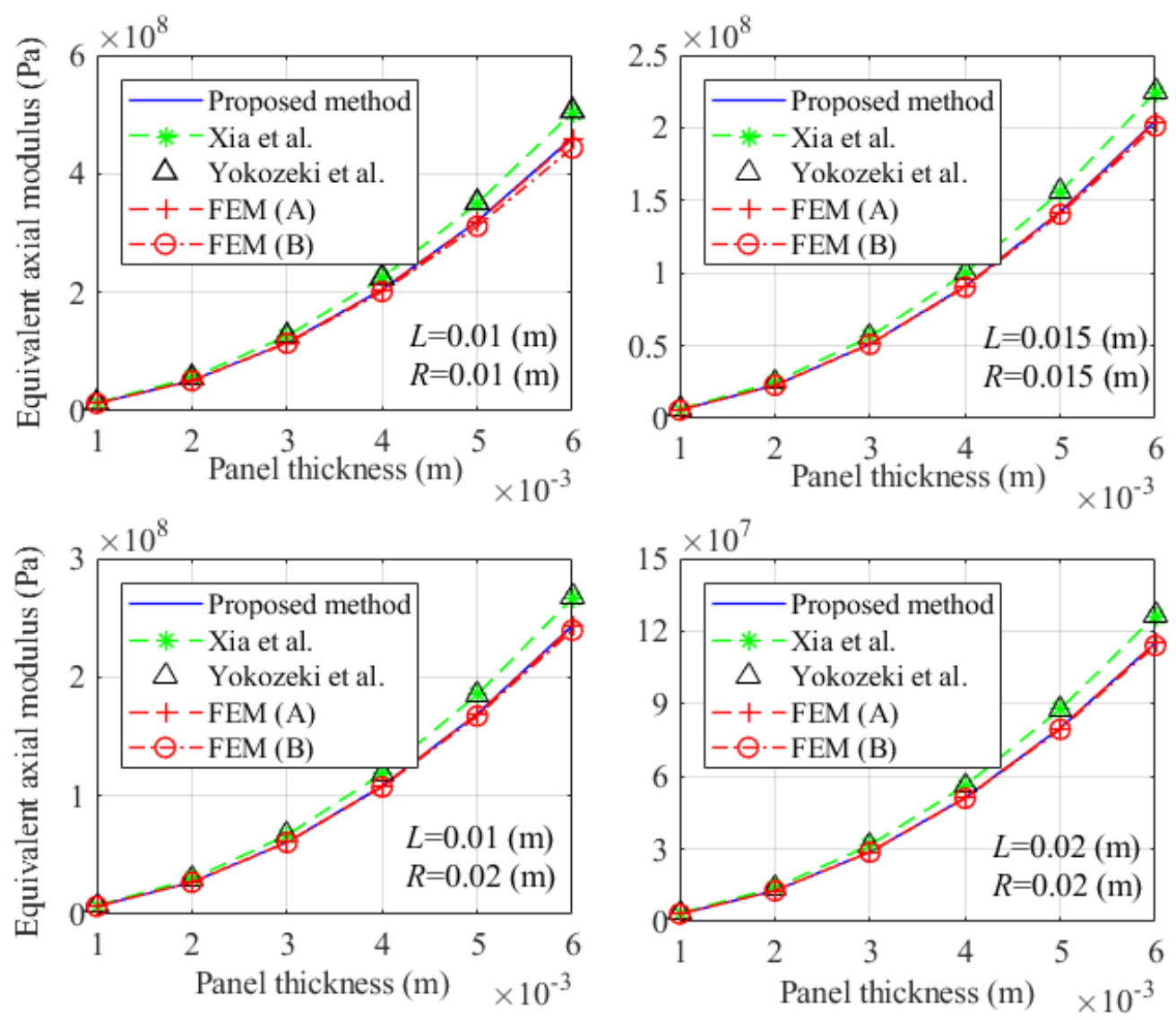

Figure 4.6 Equivalent axial modulus of the round corrugation panel

The axial equivalent modulus of the round corrugation panel is shown in Figure 4.6. The homogenous method [111] provides very close results to the method proposed by Yokozeki et al. [31]. And the proposed method generally has a smaller error compared to the finite element than the trapezoidal corrugation panel, especially for 
the Euler beam element.

The differences are due to the beam extension. The proposed method only considers the deflections caused by the bending of the beams. As shown in Figure 4.7, if the beam extension is included, the errors can be reduced significantly. Detailed analytical derivation also points out the extension has a very small influence on the vertical deflections of trapezoidal corrugation panels and both axial and vertical deflections of round corrugation panels.
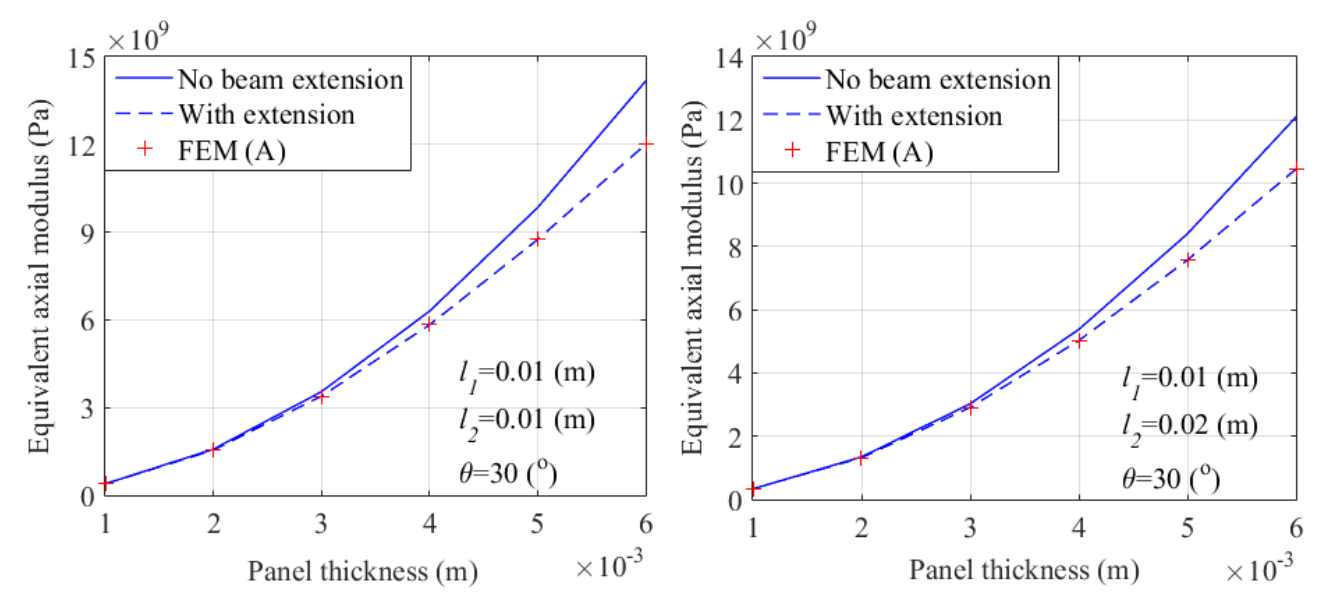

Figure 4.7 Influence of the beam extension on the equivalent axial modulus

After the numerical verification of the deflections, we can represent the coupling coefficient $C_{x w}$ between the extension force and the vertical deflection of the corrugated panel as

$$
C_{x w}=\frac{v_{n}}{T}=\frac{n \cdot v_{u n i t}}{T}
$$

For the trapezoidal corrugation

$$
C_{x w}=\frac{l_{1} \sin (\theta)\left(l_{1}+l_{2}\right)\left(l_{2}+2 l_{1} \cos (\theta)\right)}{E I} n
$$

For the round corrugation

$$
C_{x w}=\frac{2 R\left(L^{2}+\pi L R+2 R^{2}\right)}{E I} n
$$

Then the vertical deflections of the corrugation panels are verified by the finite 
element method. Each finite element model has 10 units. The Euler beam element is used in the finite element model and the extension load per width is $1 \mathrm{~N} / \mathrm{mm}$. Figure 4.8 shows a linear relationship between the vertical deflection and the unit number. Different combinations of the shape of the corrugation unit are tested with the variable $t$ representing the thickness of the corrugation panel. As shown in the figure, the analytical solution has a good agreement with results obtained by the finite element model, which verifies the analytical expressions of the vertical deflection and coupling coefficient.
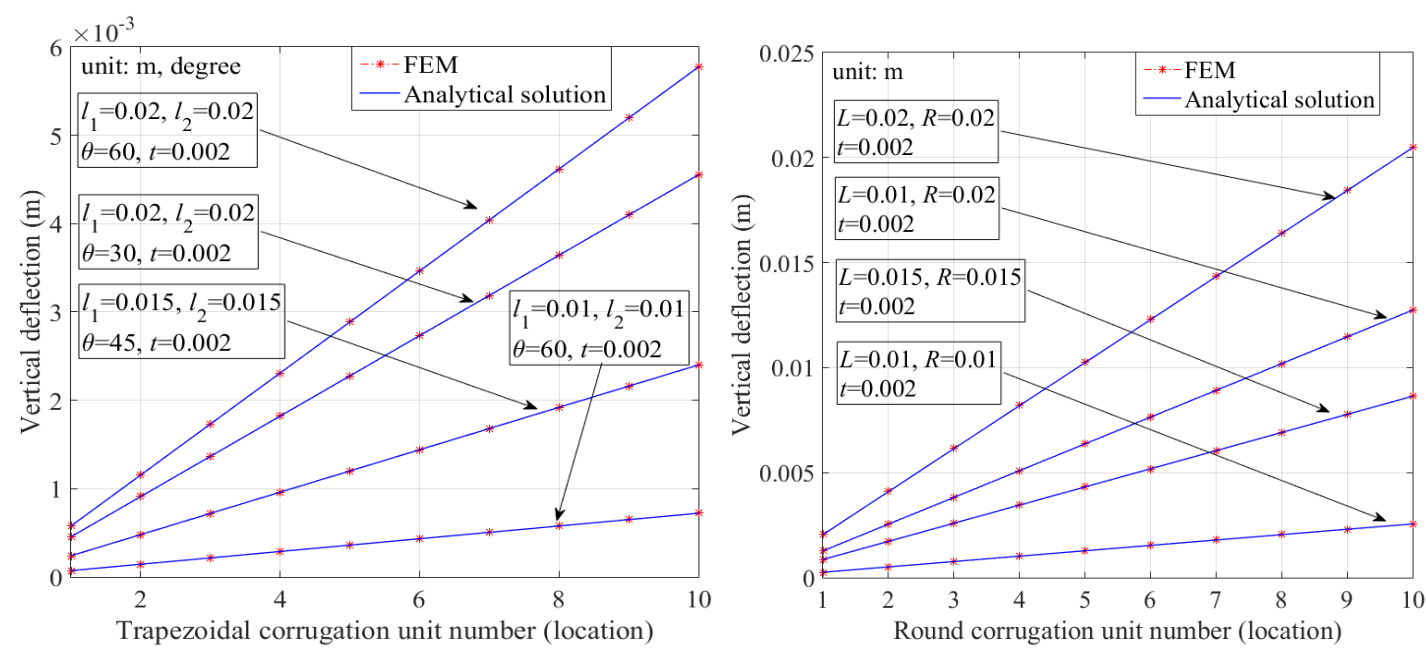

Figure 4.8 Vertical deflections verified by the FEM model, (a): Trapezoidal, (b):

Round corrugation

A general equation is used to express the axial and bending coupling effect. As shown in Figure 4.9, the corrugated panel is represented by two segments: the first corrugation unit which takes the boundary condition into account, and the second segment represents the rest of the corrugation units. Then the vertical deflection of the entire structure $v_{n}$ can be expressed as

$$
\begin{aligned}
& v_{n}=\tan \gamma_{x y} \cdot L_{n} \\
& \tan \gamma_{x y}=\frac{v_{1}}{2 c} \\
& v_{1}=v_{\text {unit }}
\end{aligned}
$$

where $L_{n}$ is the length of the corrugated panel, and $L_{n}=n \cdot 2 c$, and the vertical deflection of the first segment is denoted by $v_{1}$. 
By dividing the entire structure into two segments, the vertical deflection due to the fixed boundary condition can be represented by the rotation of the first segment. If the corrugated panel has a pinned boundary condition, no rotation angle will be generated. If the corrugated panel has a fixed boundary condition, the rotation angle $\gamma_{x y}$ of the first corrugated unit will be constant in the entire structure. This shows how the boundary condition can affect the coupling terms, which is considered a supplementary to the previous models.

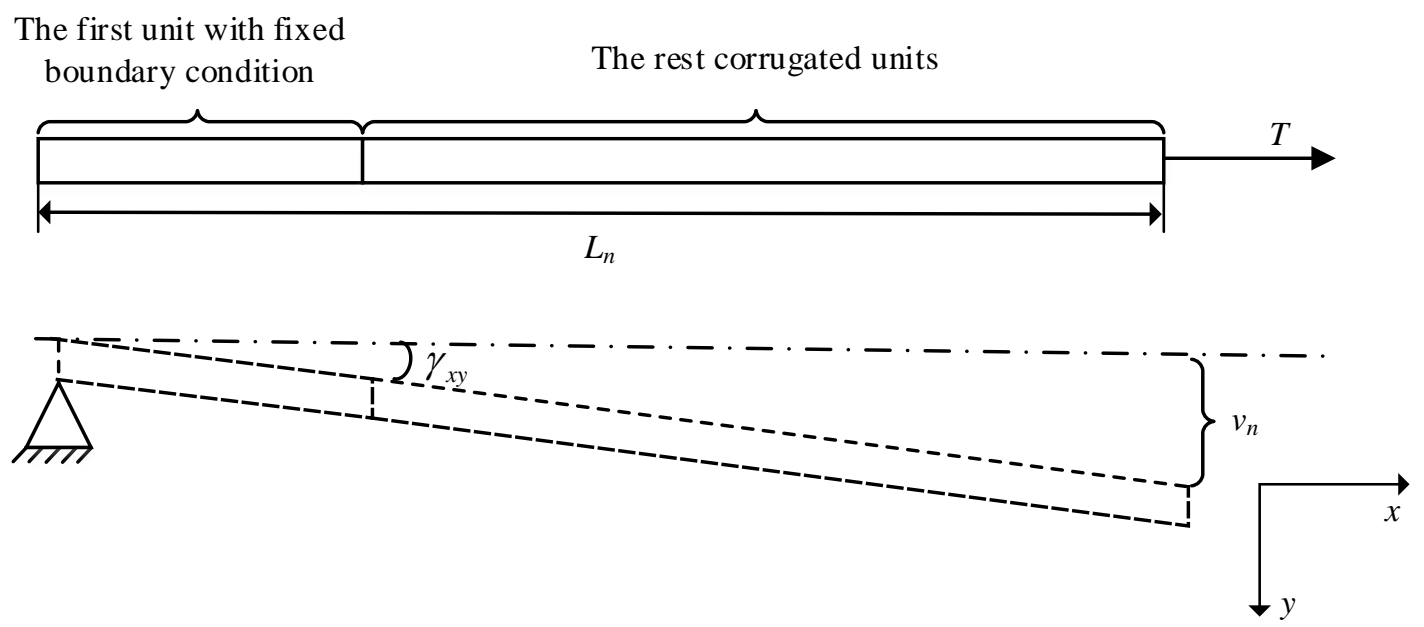

Figure 4.9 Vertical deflection under the pure extension load

A modification can be made to include the vertical deflection into the equivalent model. Another row (and column) is added to the global flexibility matrix on the basis of reference [111], in which $\bar{S}_{44}$ is the transverse component in the plane $x y$ and $\bar{S}_{14}$ is the coupling component between the loading in the $x$-direction and the shear strain in the $x y$ plane. It should be noted that the coordinate definition used in the chapter is not the same to that in the literature. 


$$
\left\{\begin{array}{l}
\bar{\varepsilon}_{x} \\
\bar{\varepsilon}_{z} \\
\bar{\gamma}_{x z} \\
\bar{\gamma}_{x y} \\
\bar{\kappa}_{x} \\
\bar{\kappa}_{z} \\
\bar{\kappa}_{x z}
\end{array}\right\}=\left[\begin{array}{ccccccc}
\bar{S}_{11} & \bar{S}_{12} & 0 & \bar{S}_{14} & 0 & 0 & 0 \\
\bar{S}_{12} & \bar{S}_{22} & 0 & 0 & 0 & 0 & 0 \\
0 & 0 & \bar{S}_{33} & 0 & 0 & 0 & 0 \\
\bar{S}_{14} & 0 & 0 & \bar{S}_{44} & 0 & 0 & 0 \\
0 & 0 & 0 & 0 & \bar{S}_{55} & \bar{S}_{56} & 0 \\
0 & 0 & 0 & 0 & \bar{S}_{56} & \bar{S}_{66} & 0 \\
0 & 0 & 0 & 0 & 0 & 0 & \bar{S}_{77}
\end{array}\right]\left\{\begin{array}{l}
\bar{N}_{x} \\
\bar{N}_{z} \\
\bar{N}_{x z} \\
\bar{N}_{x y} \\
\bar{M}_{x} \\
\bar{M}_{z} \\
\bar{M}_{x z}
\end{array}\right\}
$$

When only extension load is applied, the load factor is $\left[\begin{array}{lllllll}\bar{N}_{x} & 0 & 0 & 0 & 0 & 0 & 0\end{array}\right]^{T}$. The shear strain $\bar{\gamma}_{x y}$ is

$$
\bar{\gamma}_{x y}=\bar{N}_{x} \bar{S}_{14}
$$

Thus,

$$
\bar{S}_{14}=\frac{\bar{\gamma}_{x y}}{\bar{N}_{x}}=\frac{a \tan \left(v_{n} /(n \cdot 2 c)\right)}{\bar{N}_{x}}
$$

where $\bar{N}_{x}$ is the force per unit width of the corrugated panel.

\subsubsection{Method to eliminate the vertical deflection from extension}

In Section 4.2.3, the vertical deflection caused by the extension loads is investigated when the corrugated panel has a fixed boundary condition. However, this deflection is not desirable in some cases, such as span morphing or camber morphing applications, since the vertical deflection may change the aerodynamic shape or increase the required actuation force. A solution is provided here to eliminate the deflection by using the opposite rotation angle of the beam cross section, which can introduce an opposite vertical deflection. The opposite beam rotation is obtained by providing an offset to the initial boundary condition. As shown in Figure 4.10, the offset of the corrugated panel $l_{0}$ generates the vertical deflection $v_{0}$ and rotation angle $\alpha_{0}$.

The rotation angle $\alpha_{0}$ will lead to the vertical deflection $w_{r}$ in the opposite direction 
to $v_{n}$. Then the total vertical deflection with the offset boundary condition can be represented as

$$
\begin{aligned}
v_{\text {offset }}= & v_{0}+v_{r}+v_{n} \\
& =v_{0}+\alpha_{0} \cdot L_{n}+v_{n}
\end{aligned}
$$

For the trapezoidal corrugation,

$$
\begin{aligned}
& v_{0}=-\frac{T l_{0}^{3} \sin \theta}{3 E I} \cos \theta \\
& \alpha_{0}=-\frac{T l_{0}^{2} \sin \theta}{2 E I} \cdot n L
\end{aligned}
$$

Then,

$$
v_{\text {offset }}=-\frac{T l_{0}^{3} \sin (\theta)}{3 E I} \cos (\theta)-\frac{T l_{0}^{2} \sin \theta}{2 E I}(n \cdot 2 c)+n\left(\frac{T l_{1} \sin \theta\left(l_{1}+l_{2}\right)\left(l_{2}+2 l_{1} \cos \theta\right)}{E I}\right)
$$

Making the above equation zero will give a cubic equation of $l_{0}$. For the round corrugation, we have

$$
\begin{gathered}
w_{0}=0 \\
\alpha_{0}=-\frac{T l_{0}^{2}}{2 E I} \\
v_{\text {offset }}=-\frac{T l_{0}^{2}}{2 E I}(n \cdot 4 R)+n \frac{2 T R\left(L^{2}+2 R^{2}+\pi L R\right)}{E I}
\end{gathered}
$$

Making the above equation zero, we can obtain the offset as

$$
l_{0}=\sqrt{L^{2}+2 R^{2}+\pi L R}
$$

The offset boundary condition will also affect the axial deflection, which changes the equivalent axial modulus. For the round corrugation panel, the axial deflection of the offset part is

$$
u_{0}=\frac{T l_{0}^{3}}{3 E I}
$$

Thus, the change of the equivalent axial modulus is 


$$
\frac{u_{0}}{u_{n}}=\frac{u_{0}}{n \cdot u_{\text {unit }}}
$$
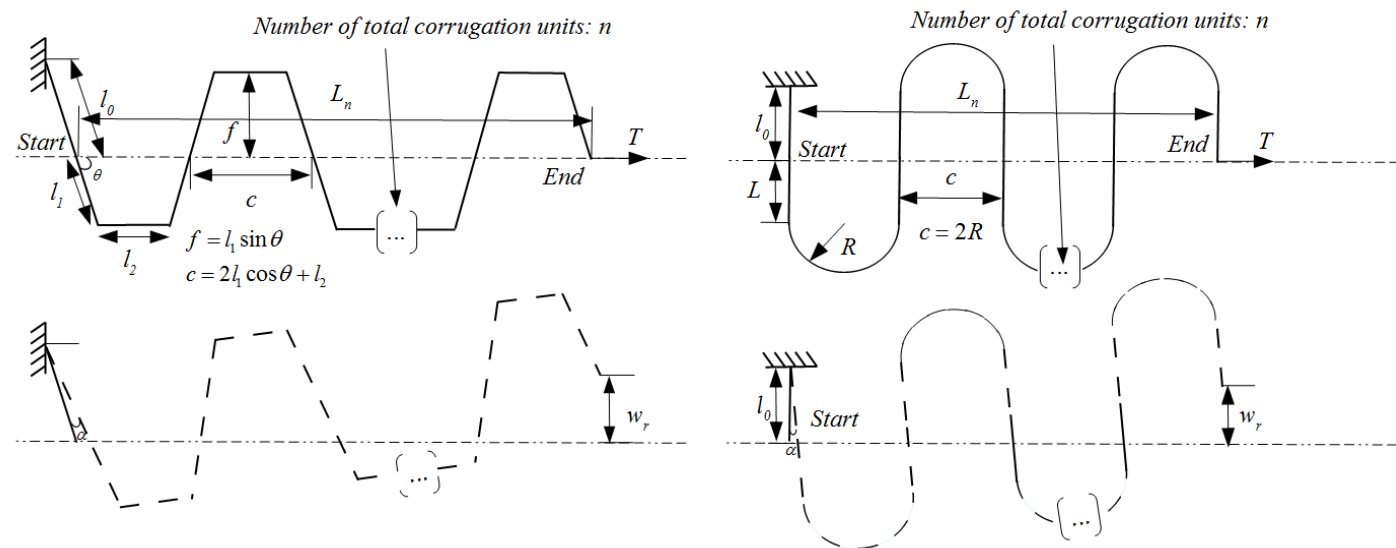

Figure 4.10 Offset boundary condition and the vertical deflection caused by the opposite rotation

\subsection{Equivalent stiffness matrix of the corrugated panels}

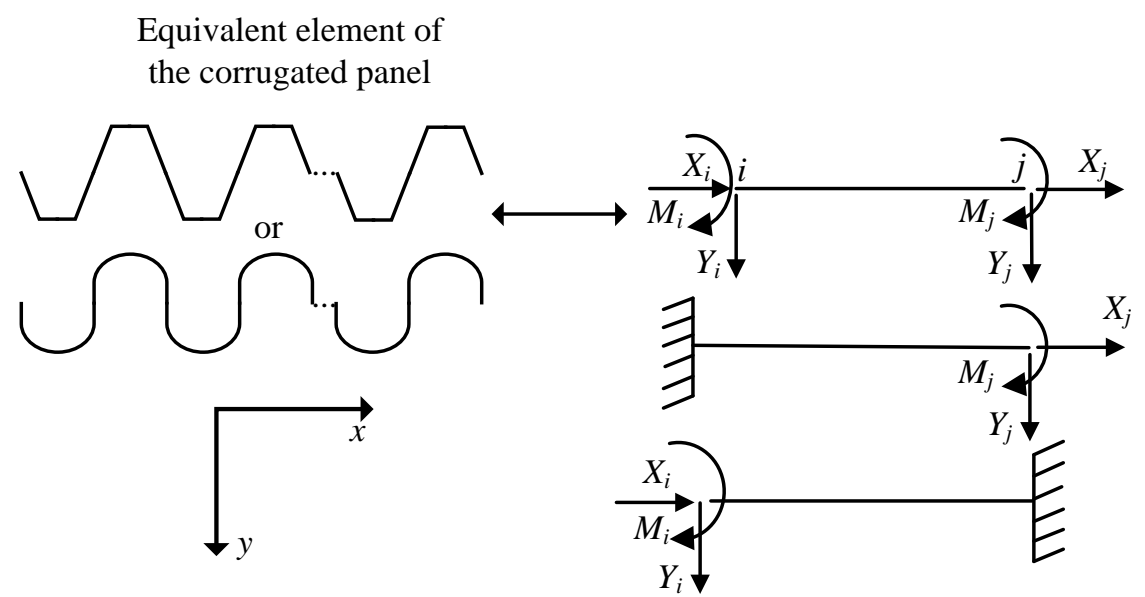

Figure 4.11 Equivalent element representing the corrugated panel

The corrugated panel can be represented by the equivalent element as shown in Figure 4.11.

The equivalent beam has two nodes, i.e., $i$ and $j$. The nodal loads are represented by $X$ (force in the $x$-direction), $Y$ (force in the $y$-direction), $M$ (moment about the $z$-direction). The nodal displacements are represented as $u$ (displacement in the $x$-direction), $v$ (displacement in the $y$-direction) and $\alpha$ (rotation angle about the 
$z$-direction) respectively. The nodal loads and deflections of each node are denoted by its subscript $i$ and $j$. The relationship between the nodal loads and the displacements can be expressed as

$$
\left[\begin{array}{c}
X_{i} \\
Y_{i} \\
M_{i} \\
X_{j} \\
Y_{j} \\
M_{j}
\end{array}\right]=\left[\begin{array}{llllll}
k_{11} & k_{12} & k_{13} & k_{14} & k_{15} & k_{16} \\
& k_{22} & k_{23} & k_{24} & k_{25} & k_{26} \\
& & k_{33} & k_{34} & k_{35} & k_{36} \\
& & & k_{44} & k_{45} & k_{46} \\
& & & & k_{55} & k_{56} \\
s y m . & & & & & k_{66}
\end{array}\right]\left[\begin{array}{c}
u_{i} \\
v_{i} \\
\alpha_{i} \\
u_{j} \\
v_{j} \\
\alpha_{j}
\end{array}\right]=K\left[\begin{array}{c}
u_{i} \\
v_{i} \\
\alpha_{i} \\
u_{j} \\
v_{j} \\
\alpha_{j}
\end{array}\right]
$$

where the stiffness matrix of the equivalent beam is denoted by $K$.

To obtain the stiffness matrix of the equivalent element, the deflections of the corrugated panel under different external loads need to be calculated first. The calculation process is similar to Section 4.2, and the internal loads of the corrugated panels caused by the axial force and the vertical force are shown in Figure 4.12 and 4.13. The internal moments caused by the axial force $X$ have been shown in Figure 4.4 and the internal loads caused by the moment are constant, and thus they are not shown in the figures. 

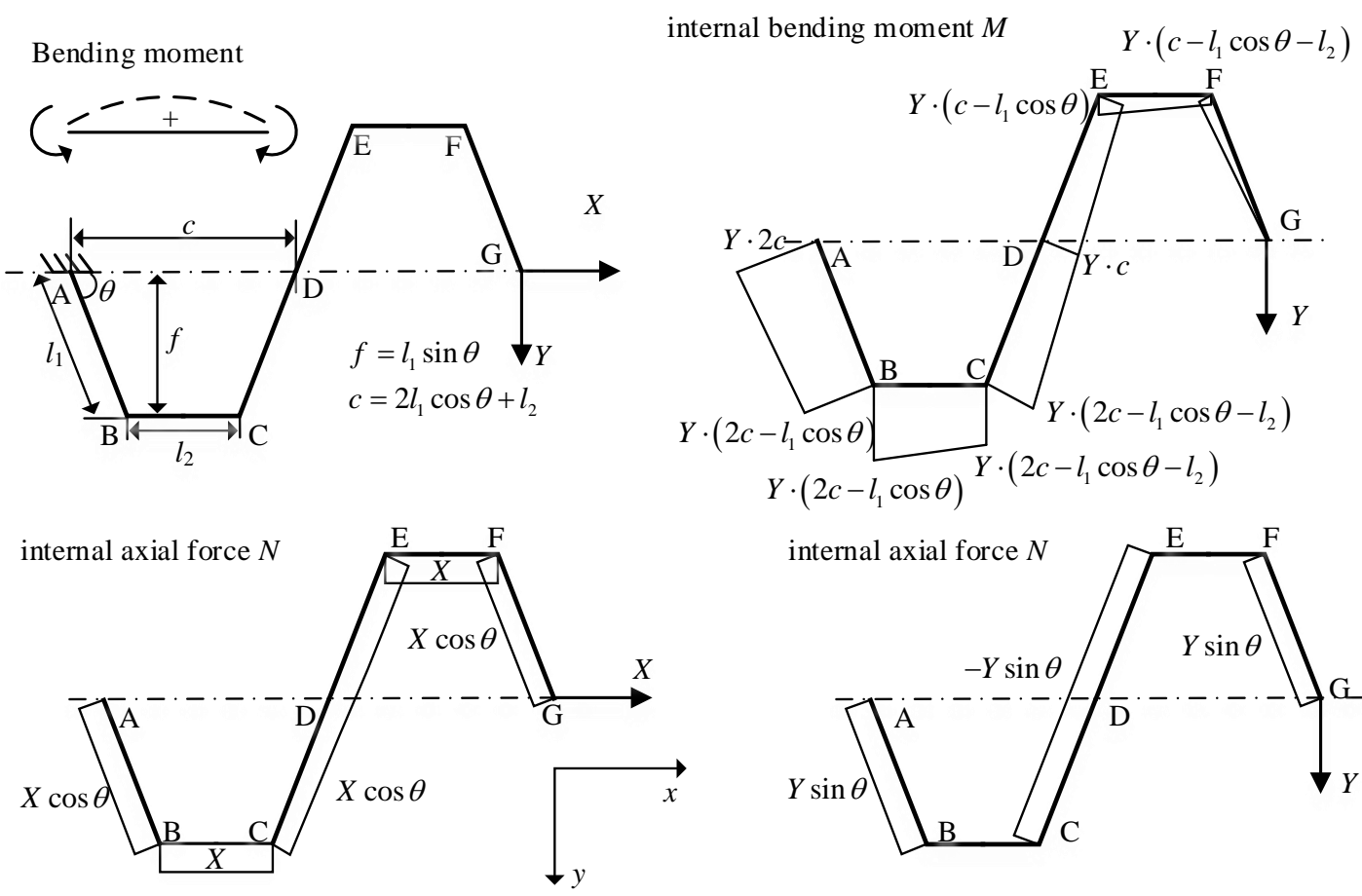

Figure 4.12 Internal loads of the trapezoidal corrugated panel under $X$ and $Y$

The deflections of the corrugated panel can be expressed as

$$
\begin{gathered}
u_{n}^{X}=\frac{X L_{n}}{\overline{E A}} \\
v_{n}^{X}=\frac{X}{K_{c}} \\
\alpha_{n}^{X}=0 \\
u_{n}^{Y}=\frac{Y}{K_{c}} \\
v_{n}^{Y}=\frac{Y L_{n}^{3}}{3 \overline{E I}}+\frac{Y}{K_{s y}} \\
\alpha_{n}^{Y}=\frac{Y L_{n}^{2}}{2 \overline{E I}} \\
u_{n}^{M}=0 \\
v_{n}^{M}=\frac{M L_{n}^{2}}{2 \overline{E I}} \\
\alpha_{n}^{M}=\frac{M L_{n}}{\overline{E I}}
\end{gathered}
$$

where the subscript ' $n$ ' denotes the number of corrugations and the superscripts $X, Y$ 
and $M$ represent the external loads causing the displacement. The length of the entire corrugated panel is represented by $L_{n}$. The terms $\overline{E A}$ and $\overline{E I}$ are the equivalent properties corresponding to the tension and bending stiffnesses, which are determined by the shape and material of the corrugated panel. The coupling coefficient between the extension force and the vertical deflection is denoted as $K_{c}$ and the coefficient between the vertical force and vertical deflection as $K_{s y}$.

For the trapezoidal corrugation, we have

$$
\begin{aligned}
& L_{n}=2 n \cdot\left(2 l_{1} \cos \theta+l_{2}\right) \\
& \overline{E A}=\frac{3\left(2 l_{1} \cos \theta+l_{2}\right) E I \cdot E A}{l_{1}^{2} \sin ^{2} \theta\left(2 l_{1}+3 l_{2}\right) E A+3\left(2 \cos ^{2} \theta l_{1}+l_{2}\right) E I} \\
& \overline{E I}=\frac{2 l_{1} \cos \theta+l_{2}}{2 l_{1}+l_{2}} E I \\
& K_{c}=\frac{E I}{l_{1} \sin \theta\left(l_{1}+l_{2}\right)\left(2 l_{1} \cos \theta+l_{2}\right) n} \\
& K_{s y}=\frac{3}{2} \frac{E I}{l_{1} l_{2}(1-\cos \theta)\left(l_{1} \cos \theta+l_{2}\right) n}
\end{aligned}
$$

For the round corrugated panel, we have

$$
\begin{aligned}
& L_{n}=n \cdot 4 R \\
& \overline{E A}=\frac{12 R \cdot E I}{4 L^{3}+6 \pi R L^{2}+24 R^{2} L+3 \pi R^{3}} \\
& \overline{E I}=\frac{2 R \cdot E I}{\pi R+2 L} \\
& K_{y x}=K_{x y}=K_{c}=\frac{1}{2} \frac{E I}{n R\left(L^{2}+\pi R L+2 R^{2}\right)} \\
& K_{s y}=\frac{3 E I}{R^{2} n(\pi R+8 L)}
\end{aligned}
$$

As shown in the Equations $(4.29-4.33)$, the force in the $x$-direction will lead to a displacement in the $y$-direction, and vice versa. The complete relationship between the nodal deflections and loads makes the stiffness matrix of the corrugated panel available, which provides a more general and compatible approach to calculate the deformation of the entire compliant structure. 
The stiffness matrix of the equivalent beam is obtained by calculating the nodal deflections when the equivalent beam has a fixed boundary condition at one node $(i$ or $j$ ) and the external load is applied at the other node $(j$ or $i)$. For example, the deflections of node $j$ are calculated under the load $\left[0,0,0, X_{j}, Y_{j}, M_{j}\right]$ when node $i$ is constrained. According to Equations (4.29 - 4.31), we have

$$
\left[\begin{array}{c}
u_{j} \\
v_{j} \\
\alpha_{j}
\end{array}\right]=\left[\begin{array}{ccc}
\frac{L_{n}}{\overline{E A}} & \frac{1}{K_{c}} & 0 \\
\frac{1}{K_{c}} & \frac{L_{n}^{3}}{3 \overline{E I}}+\frac{1}{K_{s y}} & \frac{L_{n}^{2}}{2 \overline{E I}} \\
0 & \frac{L_{n}^{2}}{2 \overline{E I}} & \frac{L_{n}}{\overline{E I}}
\end{array}\right]\left[\begin{array}{c}
X_{j} \\
Y_{j} \\
M_{j}
\end{array}\right]
$$

The loads can be obtained from Equation (4.34), by inverting the compliance matrix as

$$
\left[\begin{array}{c}
X_{j} \\
Y_{j} \\
M_{j}
\end{array}\right]=\left[K_{22}\right]\left[\begin{array}{l}
u_{j} \\
v_{j} \\
\alpha_{j}
\end{array}\right]
$$

Using the equilibrium equations, we can obtain the loads at node $i$ as

$$
\left[\begin{array}{c}
X_{i} \\
Y_{i} \\
M_{i}
\end{array}\right]=\left[K_{12}\right]\left[\begin{array}{l}
u_{j} \\
v_{j} \\
\alpha_{j}
\end{array}\right]
$$

Repeating the previous step, we can find the relationship between the node loads and deflections when the beam is under the load $\left[X_{i}, Y_{i}, M_{i}, 0,0,0\right]$. The complete relationship between the loads $\left[X_{i}, Y_{i}, M_{i}, X_{j}, Y_{j}, M_{j}\right]$ and the deflections $\left[u_{i}, v_{i}, \alpha_{i}, u_{j}\right.$, $\left.v_{j}, \alpha_{j}\right]$ can be obtained according to the principle of superposition as

$$
\left[\begin{array}{c}
X_{i} \\
Y_{i} \\
M_{i} \\
X_{j} \\
Y_{j} \\
M_{j}
\end{array}\right]=\left[\begin{array}{ll}
K_{11} & K_{12} \\
K_{21} & K_{22}
\end{array}\right]\left[\begin{array}{c}
u_{i} \\
v_{i} \\
\alpha_{i} \\
u_{j} \\
v_{j} \\
\alpha_{j}
\end{array}\right]
$$


Here, the stiffness matrix $K$ is represented by four blocks $K_{11}, K_{12}, K_{21}$ and $K_{22}$. In contrast to the normal beam element, the equivalent stiffness matrix has coupling components between the extension force $X$ and the displacement in the $y$-direction, $v$, the rotation angle, $\alpha$, due to the coupling effect.

It should also be noted that the stiffness matrix is obtained when the corrugated panel is clamped at point A as shown in Figure 4.2. If the trapezoidal corrugated panel is clamped at point $\mathrm{D}$, or the round corrugated panel is clamped at point $\mathrm{A}^{\prime}$, the displacement under extension force will be in the opposite direction, changing the stiffness matrix form of the equivalent beam, although it can still be obtained in a similar way.

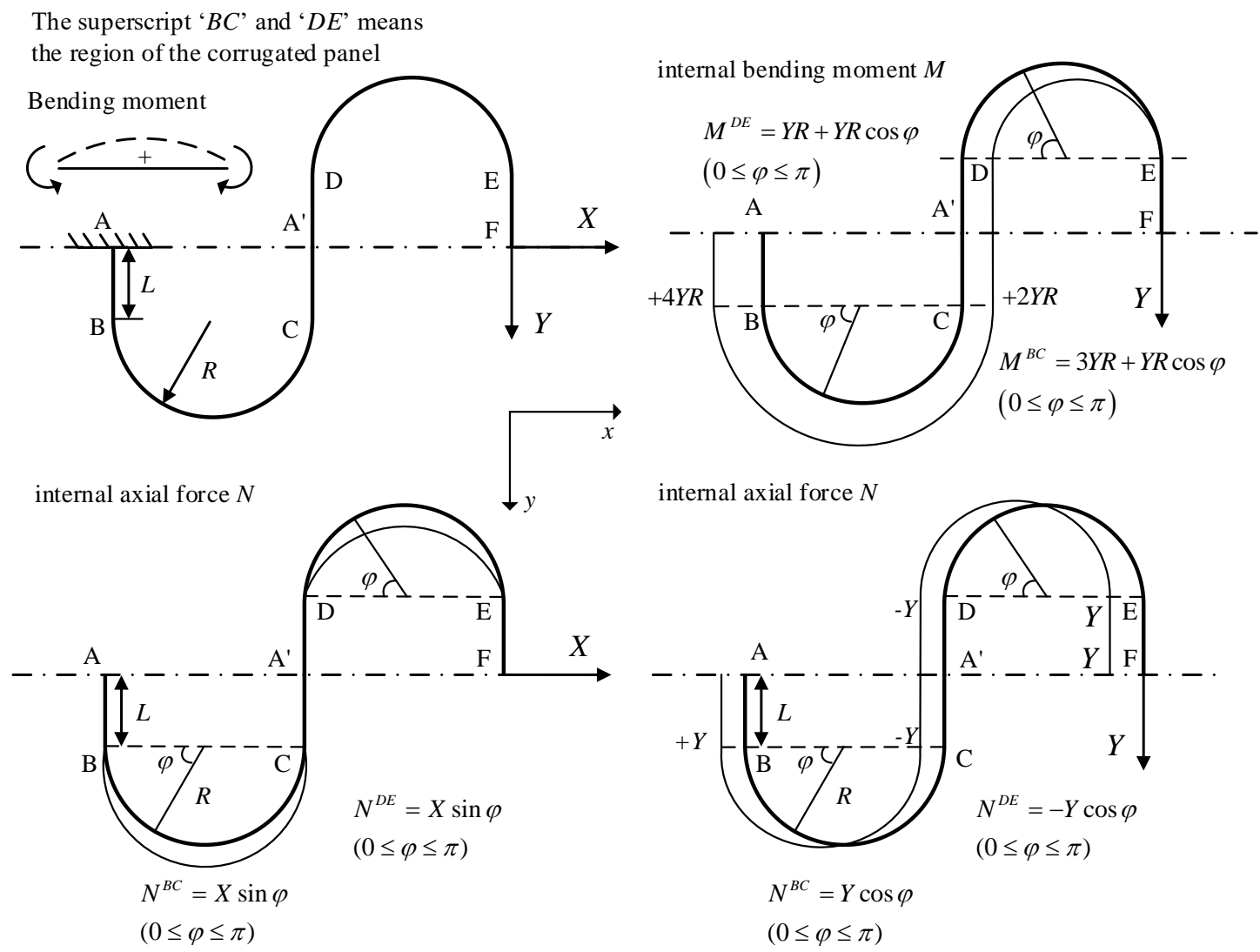

Figure 4.13 Internal loads of the round corrugated panel under $X$ and $Y$

\subsection{Deformation limits of corrugated panels}

To complete the equivalent model, the deformation limit of the corrugated panel 
needs to be calculated. Since the model employs isotropic material, the deformation limit of the corrugated panel is determined by calculating the maximum von Mises stress in the structure. The von Mises yield criterion assumes that the yielding occurs when the von Mises stress exceeds the yield stress as [117]

$$
\sqrt{\frac{1}{2}\left[\left(\sigma_{11}-\sigma_{22}\right)^{2}+\left(\sigma_{11}-\sigma_{33}\right)^{2}+\left(\sigma_{22}-\sigma_{33}\right)^{2}+6\left(\sigma_{12}^{2}+\sigma_{13}^{2}+\sigma_{23}^{2}\right)\right]}>\sigma_{y}
$$

where $\sigma_{y}$ is the yield stress of the material, and the stress component is denoted by the subscript ' 1 ', '2', ' 3 ' corresponding to the $x, y$, and $z$ axes respectively.

In the equivalent model of the corrugated panel, the von Mises stress is simplified since the model is inherently 2-dimensional and the beams in the model are assumed to be thin. Thus, all the stress components vanish except $\sigma_{11}$, and the von Mises stress is reduced to $\sigma_{11}$, which should be obtained considering both the internal axial force and bending moment, as shown in Figures 4.4, 4.12 and 4.13.

\subsection{Experimental verification by tensile test}

To verify the equivalent model, the tensile test of the corrugated panels is performed to obtain the tensile modulus of the corrugated panels and compare it with the result from the equivalent model.

The tensile test follows the ASTM-D638 standard, which is for the tensile testing of plastic material since the corrugated panels are manufactured by a 3D printer, which prints ABS plastic material. A series of samples are printed with different geometries as shown in Figure 4.14. Although the standard is not for corrugated panels, the selection of the parameters, such as the test speed, still makes the tensile tests reasonable.

The width and thickness of the corrugated panels are $0.02 \mathrm{~m}$ and $0.002 \mathrm{~m}$ respectively. The geometries are selected to provide descending tensile moduli from 'Sample-T1' to 'Sample-T6'. Each sample has two repeated corrugation units considering the manufacture limitation. The sample also has flat ends connected to the corrugation 
units, which would be clamped by the test machine. The ends have a larger thickness to ensure the sample is well clamped by the test machine and avoid cracks in the ends.

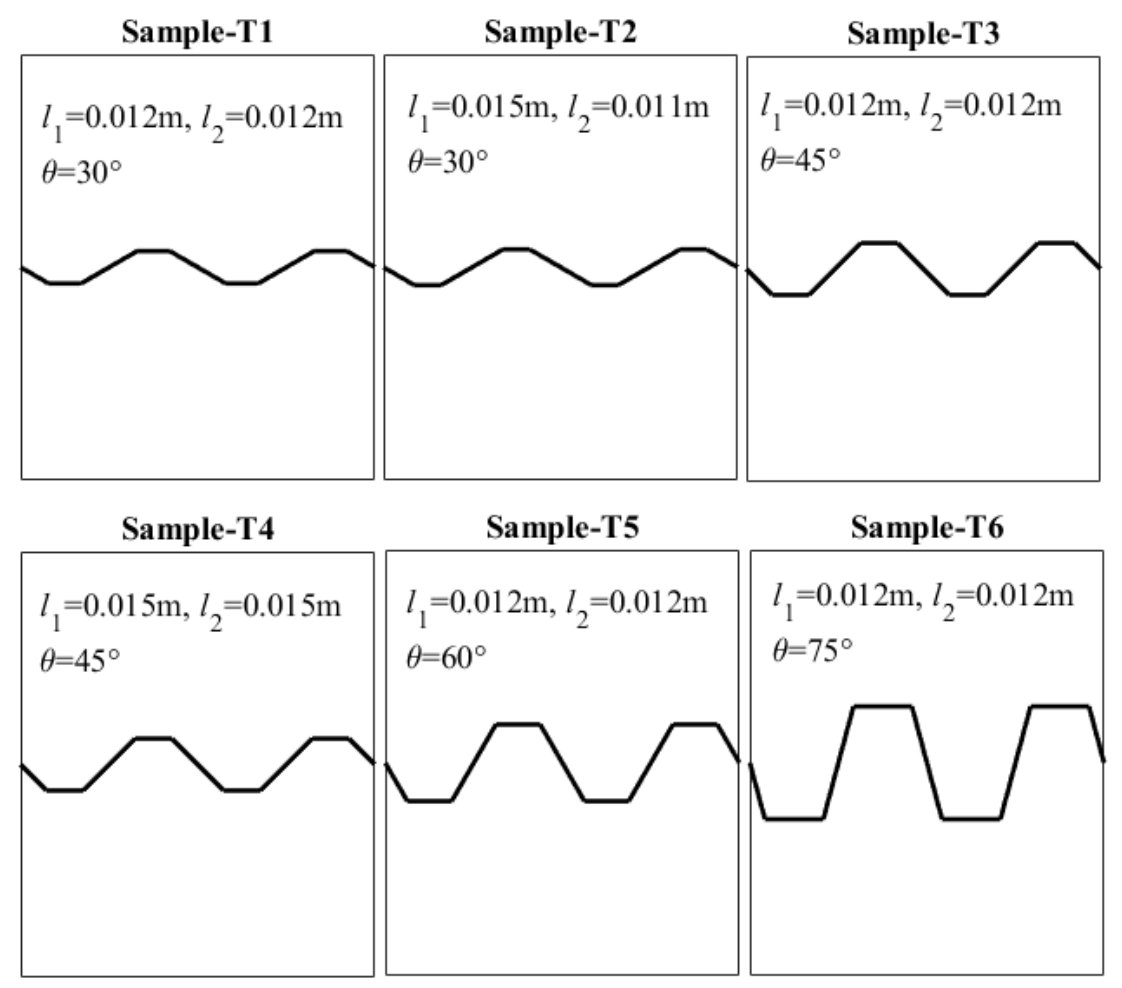

Figure 4.14 Geometry of the test samples

To predict the tensile modulus correctly from the equivalent model, good knowledge of the material property is important. However, Young's modulus of the 3D printing material is affected by the printing setup such as the density and printing pattern, which could lead to different material properties.

As seen in Equation (4.32), the equivalent tensile modulus of the corrugated panel is proportional to the first power of Young's modulus of the material, which makes it possible to eliminate its influence by the nondimensionalization of the tensile moduli of a series of corrugated panels made of the same printing material.

The tensile test is conducted on a Zwick ${ }^{\circledR}$ static testing machine. The range of the load cell is 2500 N. As shown in Figure 4.15(a), the sample is clamped at both ends. Thus, the analytical tensile modulus is obtained with the node deflections $\left[0,0,0, u_{j}\right.$, $0,0]$ to simulate the boundary conditions in the tensile test. The required tension 
force is then obtained using the stiffness matrix of the corrugated panels.

The experimental results are compared to those from the equivalent model in Figure 4.15(b). Since the corrugated panel is much more flexible under tension than its ends, the tensile deflection is assumed to be entirely introduced by the corrugated panel. The equivalent strain of the corrugated panel is calculated from the tensile deflections measured by the test machine, which is then divided by the length of the corrugated panel. In the current research, no nonlinearity is considered, and thus the test machine is set to stop the tension when the deformation of the corrugated panel reaches $5 \%$ of the length of the corrugated panel.

Two groups of corrugated panels were printed, which results in 12 test samples in total. The $x$ axis in Figure 4.15(b) represents the sample name and all the tensile moduli are nondimensionalized by their maximum, which makes the nondimensionalized $E_{x}$ start from 1. The test results are closer to the analytical results when the tensile modulus becomes small since a more flexible corrugated panel would have fewer errors of the tensile deflections. Generally, the tests do validate the equivalent model since the trend the tensile modulus is correct and the maximum error of the tensile modulus is about $10 \%$.
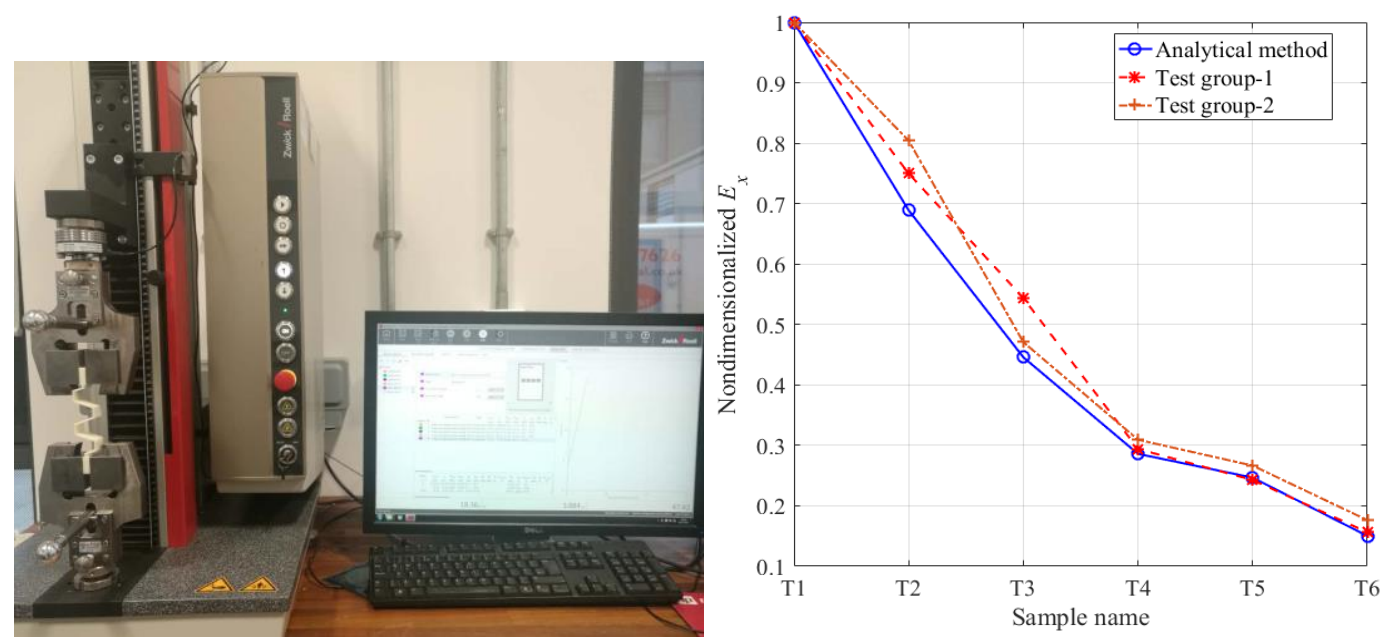

Figure 4.15 (a) Tensile test of the corrugated panel; (b) Nondimensionalized tensile modulus validation 


\subsection{Summary}

In this chapter, the equivalent model of the corrugated panel is obtained. Trapezoidal and round corrugation panels are analysed but the method can be applied to corrugated panels with other profiles. The corrugated panel is simplified as a two-dimensional frame, which is made of classical, isotropic beams and assumed to be rigid-connected. Moreover, detailed analysis of the internal loads in the corrugated panel makes the calculation of the deflections of the beams and the rotation angles of the beam cross sections available. Thus, the deflections of the points in the corrugated panels can be accumulated using the deflections of the previous points, the local deflections of the beams and rigid translation due to the beam rotations when the corrugated panel has the fixed boundary conditions at one end.

The deflection caused by the axial load is investigated first. Analytical expressions of the deflections are obtained, including the vertical deflections caused by the axial load. The deflections are verified by the existing methods in the literature and the finite element method. It is also found that the beam extension of trapezoidal panels needs to be considered for the prediction of the equivalent axial modulus. The vertical deflection caused by the axial load can be eliminated by employing an offset between the centre axis and the boundary condition, while the coupling of the vertical deflection and the axial load requires the modification of the existing models.

A general solution is provided with an equivalent beam element, which has the same overall deflections to the corresponding corrugated panel. Using the same calculation process, the deflections caused by different external loads are also obtained, which are represented by the properties of the equivalent beam. Then, the stiffness matrix of the beam is obtained, which has the coupling terms between the vertical deflections and the axial load, and vice versa. The stiffness matrix will allow the modelling of the compliant structure based on unsymmetrical stiffness, which will be 
used in Chapter 5. Since the model adopts the beam element, the optimisation of the compliant structure will be very fast, which will be shown in Chapters 5 and 6 .

A simplified method to calculate the deformation limit is also presented using the internal loads of the corrugated panels, which can be efficient and sufficient for a preliminary study.

Tensile tests of the corrugated specimens were also performed to validate the proposed equivalent model. A series of samples were tested with different geometries, which represents the change of the tensile modulus caused by the geometry of the corrugation. The tensile modulus is nondimensionalized to eliminate the influence of the material properties. The errors can also be reduced since more than 12 samples are tested in total. The results show that the equivalent model is able to represent the corrugated panel, and can be used in the following chapters for the optimisation of the compliant structure. 


\section{Chapter 5 Optimisation of the compliant structure using the equivalent model}

\subsection{Introduction}

A novel compliant structure based on stiffness asymmetry has been introduced in Chapter 3. A rotation angle of the compliant structure can be introduced by a linear actuation when the compliant structure has unsymmetrical stiffness in its different components.

Increasing the stiffness asymmetry causes a larger deformation, whereas increasing the total stiffness will enable larger aerodynamic loads to be carried. The features presented in Chapter 3 demand the optimisation to explore the design space of the unsymmetrical stiffness.

In this chapter, the stiffness asymmetry is provided by corrugated panels, which will be replaced with the equivalent model proposed in Chapter 4. Beam elements are used together with the equivalent model to assemble the stiffness matrix of the entire compliant structure.

An optimisation is performed to find the optimum geometry variables of the corrugated panels. The actuation force is minimised with a required rotation and aerodynamic loads, and constraints of the geometry and structural material. The influence of the geometry parameters on the optimum results is shown, which indicates the different features of round and trapezoidal corrugated panels. The optimal results determine the detailed designs in each case. Optimisation cases are demonstrated on a single compliant structure as well as the compliant structure consisting of multiple units, including a case study under fixed span and 
aerodynamic loads and a case study of an aircraft with fewer constraints, which highlights the improved system level performance compared to the case study in Chapter 3.

\subsection{Equivalent model of the compliant structure}

\subsubsection{Analytical method}

The analytical method to calculate the vertical deflection of the compliant structure is first introduced. As expressed in Chapter 3 and 4, the vertical deflection of the compliant structure under the actuation force $F$ will consist of two components, namely the vertical deflection caused by the stiffness asymmetry of the corrugated panels, and the vertical deflection due to coupling coefficient $C_{x w}$ between the extension force and the vertical deflection of the corrugated panel.

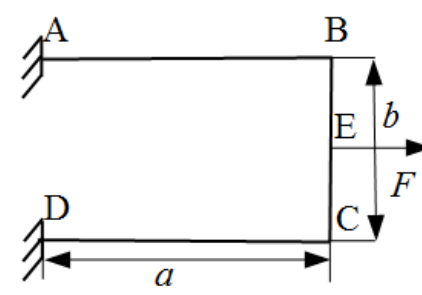

(1)

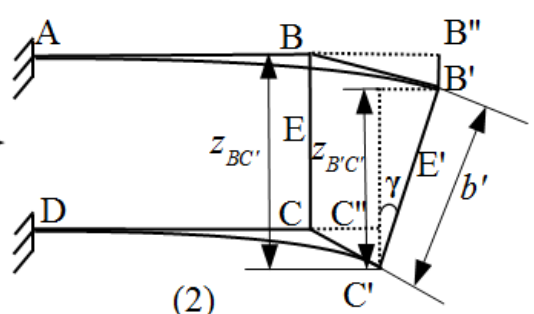

(2)

Figure 5.1 Geometry relationship in the compliant structure

According to Equations (3.11), (3.14), and (3.15), the vertical deflections of point B, $\mathrm{C}$ and $\mathrm{E}$ due to the stiffness asymmetry is

$$
\begin{aligned}
& v_{B}=v_{C}=v_{E}=\frac{F b}{2} a^{2} \frac{E_{2} A_{2}-E_{1} A_{1}}{2 K_{t}} \\
& K_{t}=E_{1} A_{1} E_{1} I_{1}+E_{2} A_{2} E_{2} I_{2}+E_{1} A_{1} E_{2} I_{2}+E_{1} I_{1} E_{2} A_{2}+E_{1} A_{1} E_{2} A_{2} b^{2}
\end{aligned}
$$

As shown in Figure 5.1, the vertical deflections of point B and $C$, i.e., $B^{\prime \prime} B^{\prime}$ and $C^{\prime \prime} C^{\prime}$, satisfy the relationships

$$
\begin{aligned}
& z_{B^{\prime} C^{\prime}}=b^{\prime} \cos \gamma \\
& z_{B^{\prime} C^{\prime}}=z_{B C^{\prime}}-B^{\prime \prime} B^{\prime}=b+C^{\prime \prime} C^{\prime}-B^{\prime \prime} B^{\prime}
\end{aligned}
$$


Then we have

$$
C^{\prime \prime} C^{\prime}-B^{\prime \prime} B^{\prime}=b^{\prime} \cos \gamma-b
$$

where $b^{\prime}$ is the deformed length of $\mathrm{BC}$, and $\gamma$ is the inclined angle of the middle beam $\mathrm{BC}$. If the middle beam $\mathrm{BC}$ is assumed to be rigid, and the inclined angle is small, we have

$$
C^{\prime \prime} C^{\prime}=B^{\prime \prime} B^{\prime}
$$

which means the vertical deflections of point $\mathrm{B}$ and $\mathrm{C}$ are equal.

Thus the vertical deflections of points B and C caused by its extension force, $v_{B M}$ and $v_{C M}$, are also the same. According to Equation (4.13) we have

$$
\begin{aligned}
& v_{B M}=C_{x w}^{u p p} A X_{1} \\
& v_{C M}=C_{x w}^{\text {low }} X_{1}
\end{aligned}
$$

where $C_{x w}$ is the coupling coefficient of the corrugated panel. The superscript 'upp' and 'low' correspond to the upper and lower corrugated panels. $A X_{1}$ and $X_{1}$ are the extension forces in the two corrugated panels. Since $A X_{1}+X_{l}=F$, and $v_{B M}=v_{C M}$, we can deduce the vertical deflection of point $E$ due to the extension force $F$ is

$$
v_{E M}=\frac{v_{B M}+v_{C M}}{2}=\left(\frac{C_{x w}^{u p p} C_{x w}^{l o w}}{C_{x w}^{u p p}+C_{x w}^{l o w}}\right) F
$$

From Equation (5.6), the equivalent compliance of the compliant structure is essentially that of two parallel springs, which have the compliances $C_{x w}^{u p p}$ and $C_{x w}^{\text {low }}$. The final vertical deflections of the compliant structure can then be obtained by combining the deflections of the two components from Equations (5.1) and (5.6).

The analytical method is compared to the detailed finite element models, as well as the analytical method, which does not consider the vertical component caused by the extension coupling. The finite element analysis is performed in Abaqus®. A rigid body constraint is applied to the vertical beam to simulate its rigidity. Mesh convergence has also been verified before the analysis and the general purpose shell element $S 4 R$ in Abaqus is used [108]. The current analytical method is labelled as 
'Method A' and the analytical solution, which does not take the coupling effect into account, is labelled as 'Method B' in Figure 5.2.
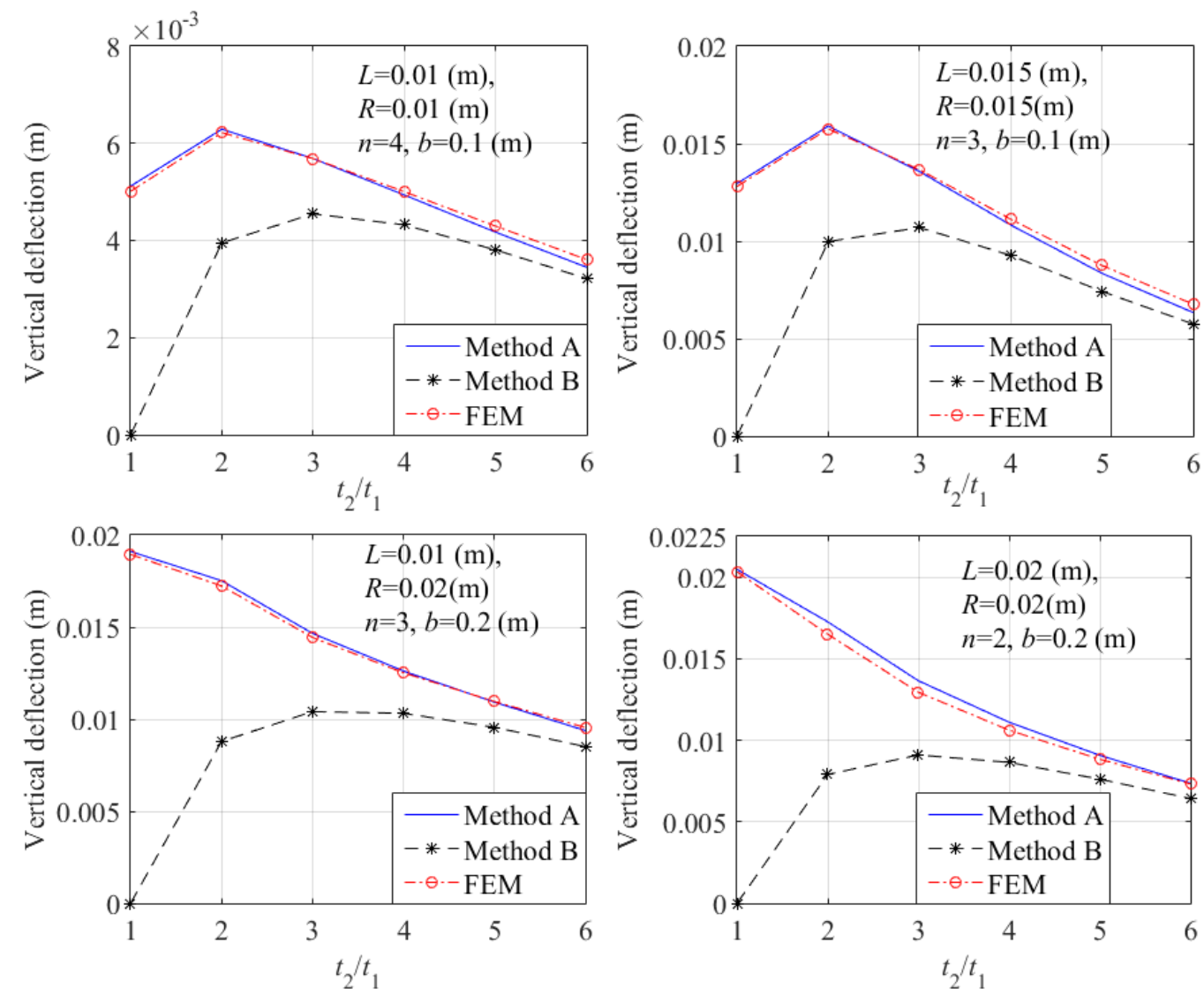

Figure 5.2 Vertical deflections of the compliant structure calculated by the analytical method

Figure 5.2 shows four different cases with different combinations of shapes and number of units. The corrugated panel is assumed to be made of Aluminum with the Young's modulus 72GPa. The variables $t_{1}$, and $t_{2}$ are the thicknesses of the upper and lower corrugated panels. The compliant structure is actuated by the force $F=100$ N, and $t_{1}$ is equal to $0.002 \mathrm{~m}$. The width of the structure is fixed at $0.01 \mathrm{~m}$. The $x$-axis represents the change of the ratio of the lower and upper panel thickness, which indicates the change of the stiffness asymmetry. We can conclude the Method A is able to predict the vertical deflections much more accurately than the Method B, especially when the upper and lower corrugation panel has the same, or close stiffness. It is found that the vertical deflection due to extension force can be even 
larger than the maximum deduced deformation from stiffness asymmetry in some cases when the height of the compliant structure is $0.2 \mathrm{~m}$, which highlights the importance of taking the coupling effect into account in this application.

The results show the analytical method can predict the vertical deflections caused when the compliant structure is actuated, while the method is constrained to the load case when only the actuation force is applied. When the aerodynamic force is applied, additional vertical deflections will also be caused, which cannot be simplified to the model represented in this section. The complicated load cases demand considering the general load cases, which leads to the stiffness matrix method given in the next section.

\subsubsection{Stiffness matrix method}

Since the equivalent stiffness matrix of the corrugated panel has been obtained in Chapter 4, the compliant structure can be represented by the equivalent model as shown in Figure 5.3(a), which consists of the equivalent elements representing the corrugated panels and the normal beam elements representing the connection part in the 2-dimensional situation.
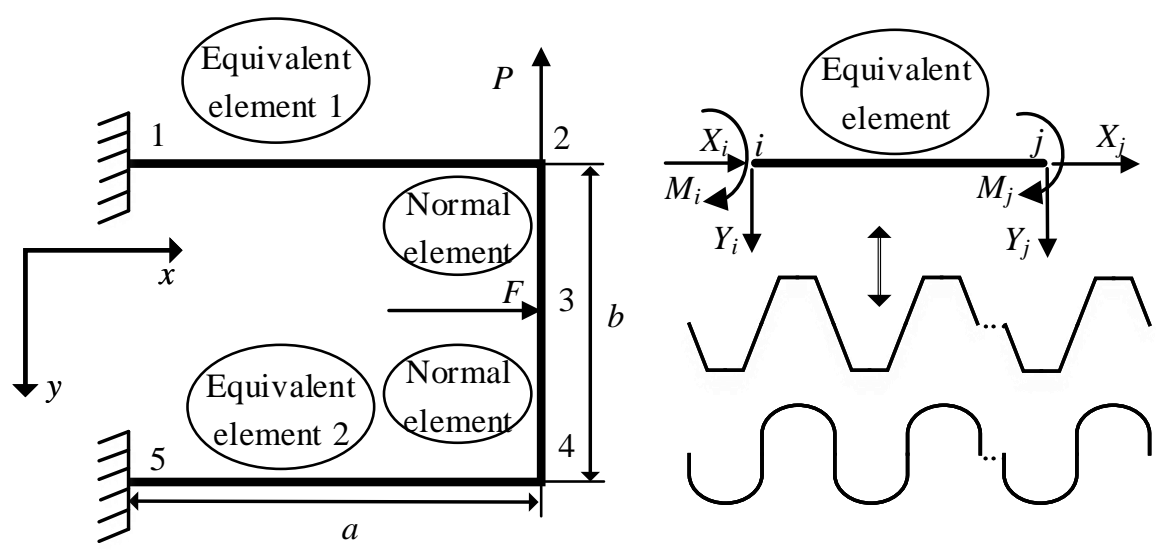

Figure 5.3 Equivalent model of the compliant structure; (b) Equivalent beam element representing the corrugated panel

The normal element is an Euler beam element, which takes both axial extension and bending into account. The stiffness matrix of the beam element is 


$$
\left[\begin{array}{c}
X_{i} \\
Y_{i} \\
M_{i} \\
X_{j} \\
Y_{j} \\
M_{j}
\end{array}\right]=\left[\begin{array}{cccccc}
E A / l & 0 & 0 & -E A / l & 0 & 0 \\
& 12 E I / l^{3} & 6 E I / l^{2} & 0 & -12 E I / l^{3} & 6 E I / l^{2} \\
& & 4 E I / l & 0 & -6 E I / l^{2} & 2 E I / l \\
& & & E I / l & 0 & 0 \\
& & & & 12 E I / l^{3} & -6 E I / l^{2} \\
& & & & &
\end{array}\right]\left[\begin{array}{c}
u_{i} \\
v_{i} \\
\alpha_{i} \\
u_{j} \\
v_{j} \\
\alpha_{j}
\end{array}\right]
$$

where $E, A, I$ and $l$ represents the Young's modulus of the material, area, the second moment of area, and the length of the beam element respectively. The subscripts $i$ and $j$ are still used to denote the two nodes of the element. The beam is assumed to be a thin beam, where the thickness is less than one-fifth of its length.

Each node of the beam element has the same degrees of freedom (the axial deflection $u$, the vertical deflection $v$ and the rotation $\alpha$ ) to the equivalent beam element, which makes the assembly of the entire stiffness matrix straightforward. The connection part between the two equivalent elements consists of two normal beam elements, and are joined at node 3, where the actuation force $F$ is applied. In this case, the location of the actuation force can be determined by the length of the two elements.

The stiffness matrix of the equivalent model is expressed in Equations (4.32)-(4.37). In total, only four elements are required with five nodes in total, which will significantly reduce the calculation time and allow a faster optimisation compared to detailed finite element analysis. The deformation of the compliant structure caused by different load cases can all be obtained simultaneously, which makes the stiffness matrix method much more efficient compared to the analytical method. Moreover, the internal loads in each element can also be obtained, enabling the calculation of the deformation limit.

In the following sections, a subscript and superscript based nomenclature method is employed to name the geometry variables in the compliant structures. For instance, the variable, ${ }^{i} X_{r}^{j}$, in which $X$ is the variable name, the superscript before the variable 
name corresponds to the upper $(i=1)$ or lower $(i=2)$ corrugated panels, the superscript $j$ corresponds to the unit number of the compliant structure if multiple units of compliant structures are applied, and the subscript $r$ is a local parameter particularly allocated for the inclined $(r=1)$ and horizontal $(r=2)$ beams of the trapezoidal corrugated panels as shown in Figure 4.2(a). Different to the previous cases, the script corresponding the upper and lower part is denoted by the superscript ' $i$ ' before the variable rather than the subscript ' $r$ ' due to the definition of the trapezoidal corrugation.

\subsubsection{Verification of the stiffness matrix method}

\subsubsection{Finite element analysis}

To verify the equivalent stiffness matrix, parametric studies are conducted, and the results are compared to those obtained from detailed finite element analysis. Three calculation cases are used for the verification for round and trapezoidal corrugations.

The finite element analysis is conducted in the commercial software Abaqus®. S4R shell elements are used in the detailed models since the S4R element has a good accuracy for both thin and thick plates [108]. The mesh size is $0.001 \mathrm{~m}$. Since the length, height and width of the compliant structure is $0.12 \mathrm{~m}, 0.1 \mathrm{~m}$ and $0.01 \mathrm{~m}$ respectively for case $1,0.16 \mathrm{~m}, 0.2 \mathrm{~m}$ and $0.01 \mathrm{~m}$ for case 2 , and $0.204 \mathrm{~m}, 0.08 \mathrm{~m}$, $0.01 \mathrm{~m}$ for case 3 , the mesh size is sufficiently small to ensure convergence.

Figure 5.4(a) shows the mesh of the Abaqus model and Figure 5.4(b) shows the vertical deflections obtained.

The geometry variables of the round corrugated panels are represented by ${ }^{1} L,{ }^{1} R$ and ${ }^{2} L,{ }^{2} R$. The thicknesses are denoted by ${ }^{1} t$ and ${ }^{2} t$ respectively. The numbers of the corrugation units of the two corrugated panels are denoted by ${ }^{1} n$ and ${ }^{2} n$ respectively. The stiffness asymmetry is achieved by changing the thickness of one panel $\left({ }^{2} t\right)$. The material's Young's modulus and the Poisson's ratio of the material are 72GPa and 0.3 respectively. Both the actuation force and the aerodynamic force are taken into 
account for the two cases. The actuation force is applied to the middle of the outboard beam, so that node 3 is equidistant from nodes 2 and 4 .

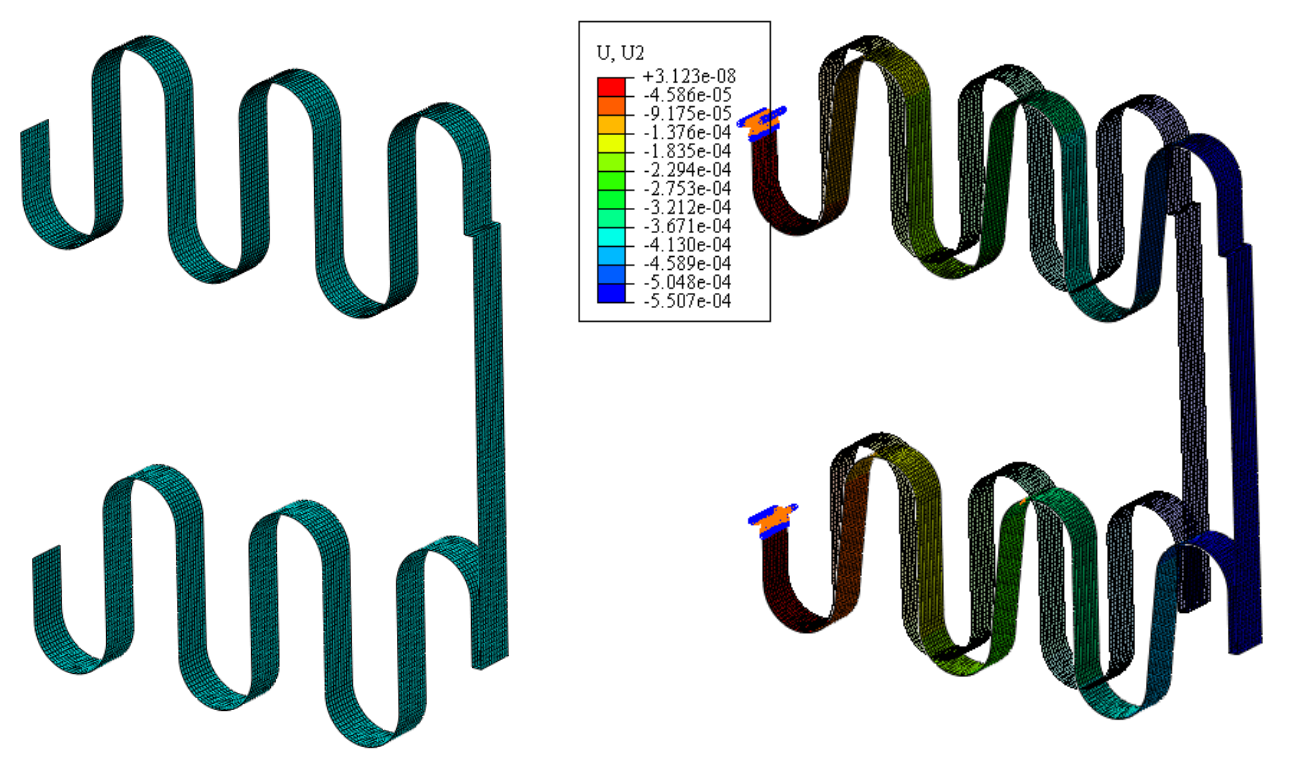

Figure 5.4 Round corrugation: (a) Mesh of the finite element model, (b) An example of the vertical deflections obtained

The vertical deflection of node 3 is obtained for the comparison. The error between the stiffness matrix method and the detailed Abaqus ${ }^{\circledR}$ analysis is below $3 \%$. However, the efficiency of the analysis is improved significantly by using the equivalent beam model of the corrugated panel. For example, the CPU time of each computation is 7.3 seconds for the detailed Abaqus analysis in Figure 5.4, while the CPU time is only 0.2 seconds for the equivalent model written in Matlab. The computation is performed with a Xeon E3 workstation with 32-GB memory.

In Figure 5.5, the vertical deflection under the actuation force increases first before it drops. The increase is due to the stiffness asymmetry caused by the different panel thickness. The coupling effect between the extension force and the vertical deflection is shown by the vertical deflection when the ratio ${ }^{2} t l^{l} t$ is 1 since the extension stiffnesses of the two corrugated panels are the same at this point. 

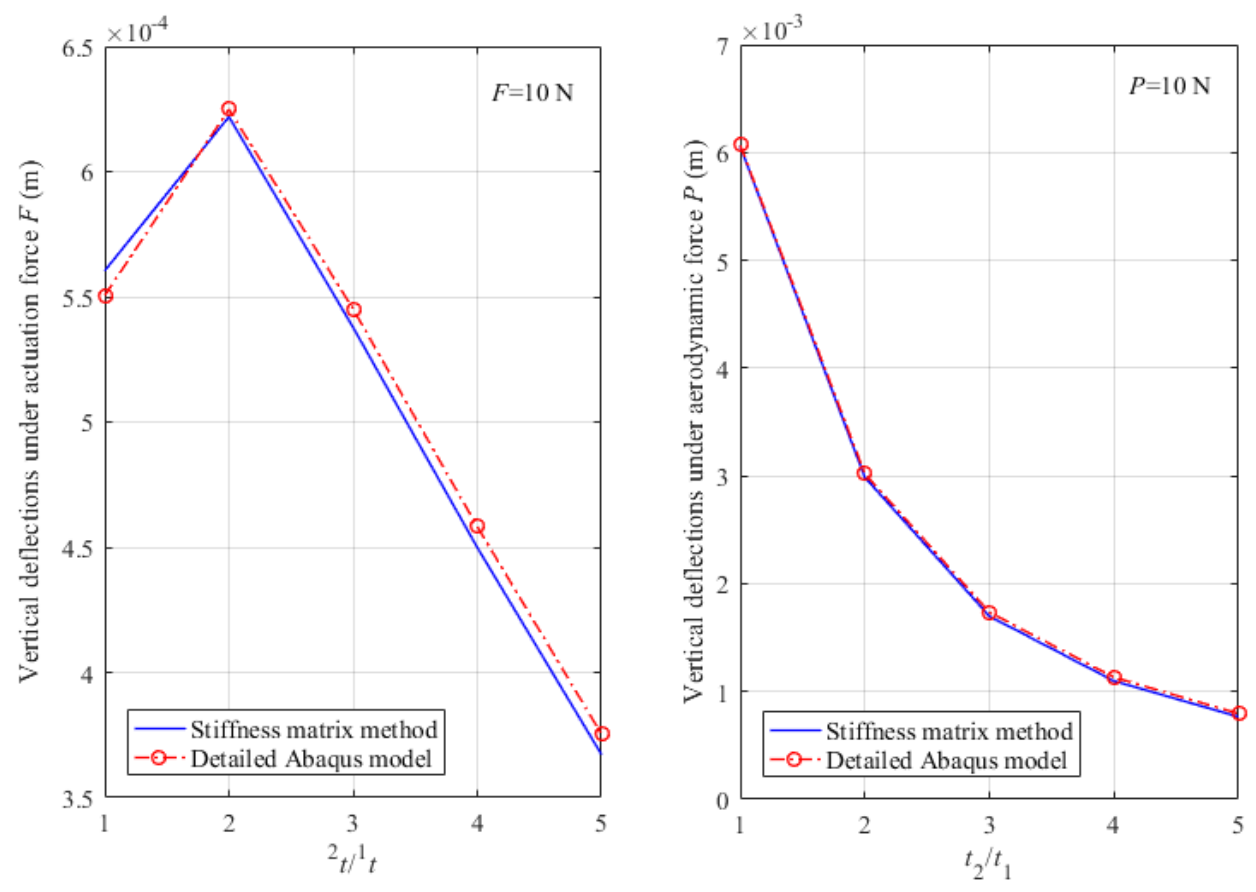

Figure 5.5 Verification of the compliant structure equivalent model by detailed finite element analysis: case $1\left(a=0.12 \mathrm{~m}, b=0.1 \mathrm{~m}, w=0.01 \mathrm{~m},{ }^{1} R=0.01 \mathrm{~m},{ }^{1} L=0.015 \mathrm{~m}\right.$,

$$
\left.{ }^{2} R=0.01 \mathrm{~m},{ }^{2} L=0.015 \mathrm{~m},{ }^{1} t=0.002 \mathrm{~m},{ }^{1} n=3,{ }^{2} n=3\right) .
$$

The vertical deflection caused by the coupling can be even larger than that caused by the stiffness asymmetry as shown in Figure 5.6, where the vertical deflection under actuation force is reduced continuously even if the stiffness asymmetry increases. The vertical deflection under aerodynamic force always decreases due to the increase in the total stiffness.

The compliant structures based on the trapezoidal corrugated panels are also verified as shown in Figure 5.7. The error is also below 3\%, which presents good compatibility of the stiffness matrix method. 

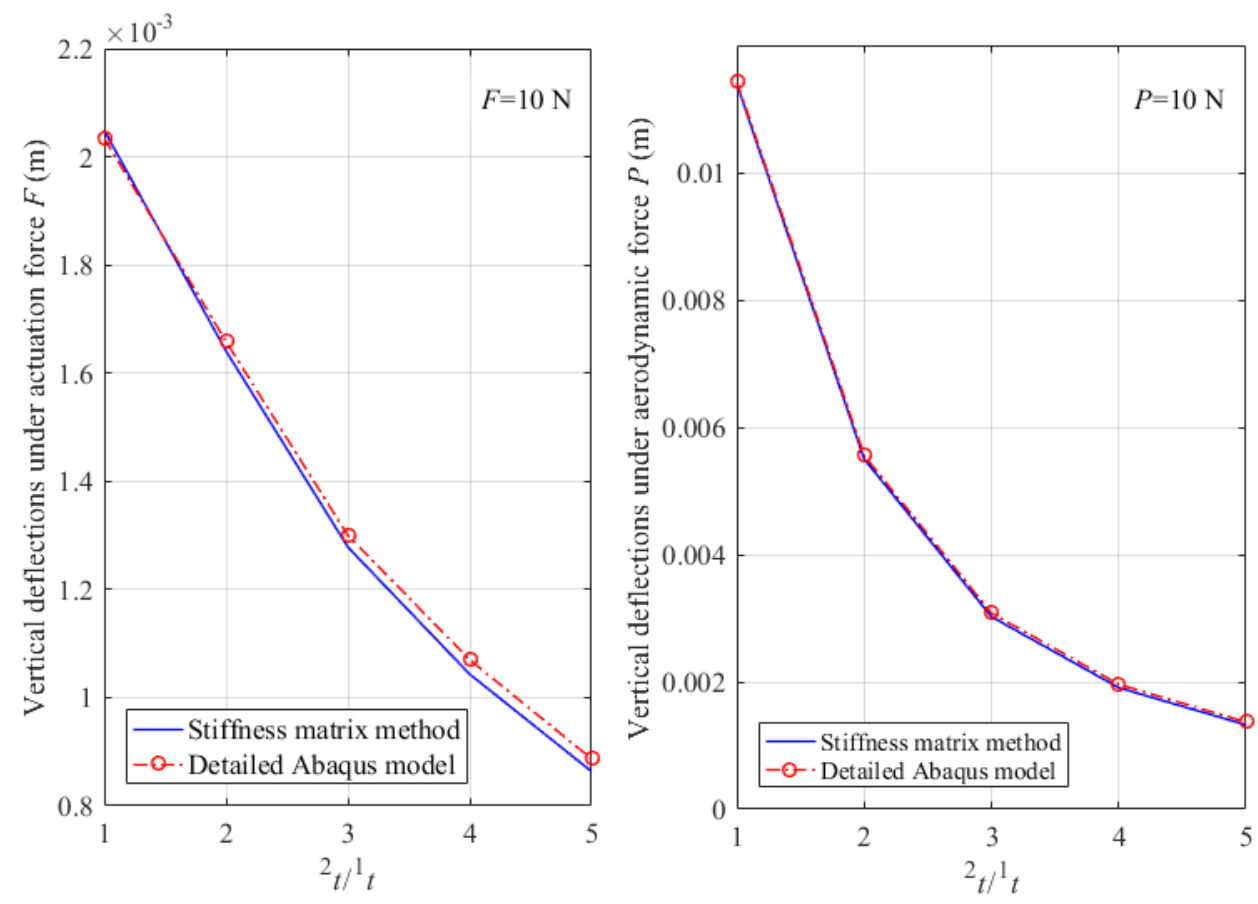

Figure 5.6 Verification of the compliant structure equivalent model by detailed finite element analysis: case $2\left(a=0.16 \mathrm{~m}, b=0.2 \mathrm{~m}, w=0.01 \mathrm{~m},{ }^{1} R=0.02 \mathrm{~m},{ }^{1} L=0.02 \mathrm{~m}\right.$, $\left.{ }^{2} R=0.02 \mathrm{~m},{ }^{2} L=0.02 \mathrm{~m},{ }^{1} t=0.002 \mathrm{~m},{ }^{1} n=2,{ }^{2} n=2\right)$
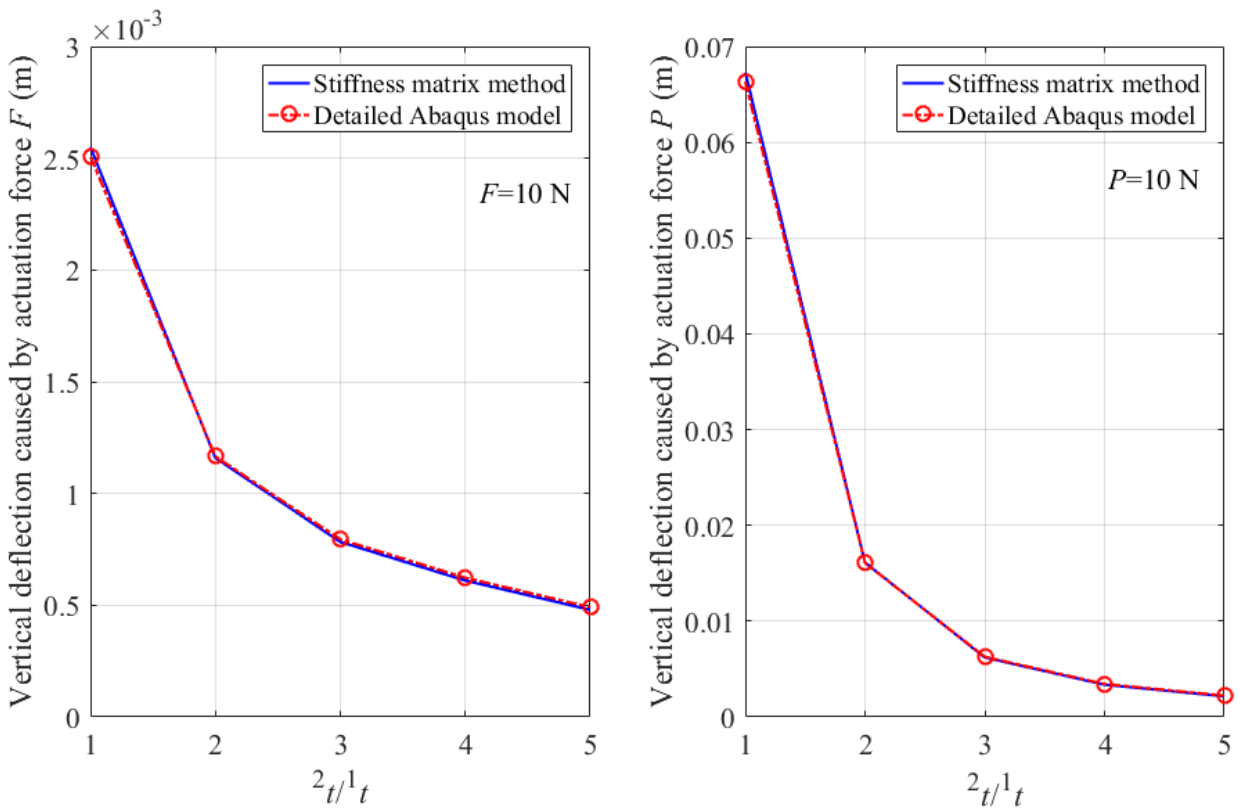

Figure 5.7 Verification of the compliant structure equivalent model by detailed finite element analysis: case 3(trapezoidal corrugated panels: $a=0.204 \mathrm{~m}, b=0.08 \mathrm{~m}$, $w=0.01 \mathrm{~m},{ }^{1} l_{1}=0.015 \mathrm{~m},{ }^{1} l_{2}=0.025 \mathrm{~m},{ }^{1} \theta=30{ }^{\circ},{ }^{1} t=0.001 \mathrm{~m}^{2} l_{1}=0.015 \mathrm{~m},{ }^{2} l_{2}=0.025 \mathrm{~m}$, $\left.{ }^{2} \theta=30^{\circ},{ }^{1} n=2,{ }^{2} n=2\right)$ 


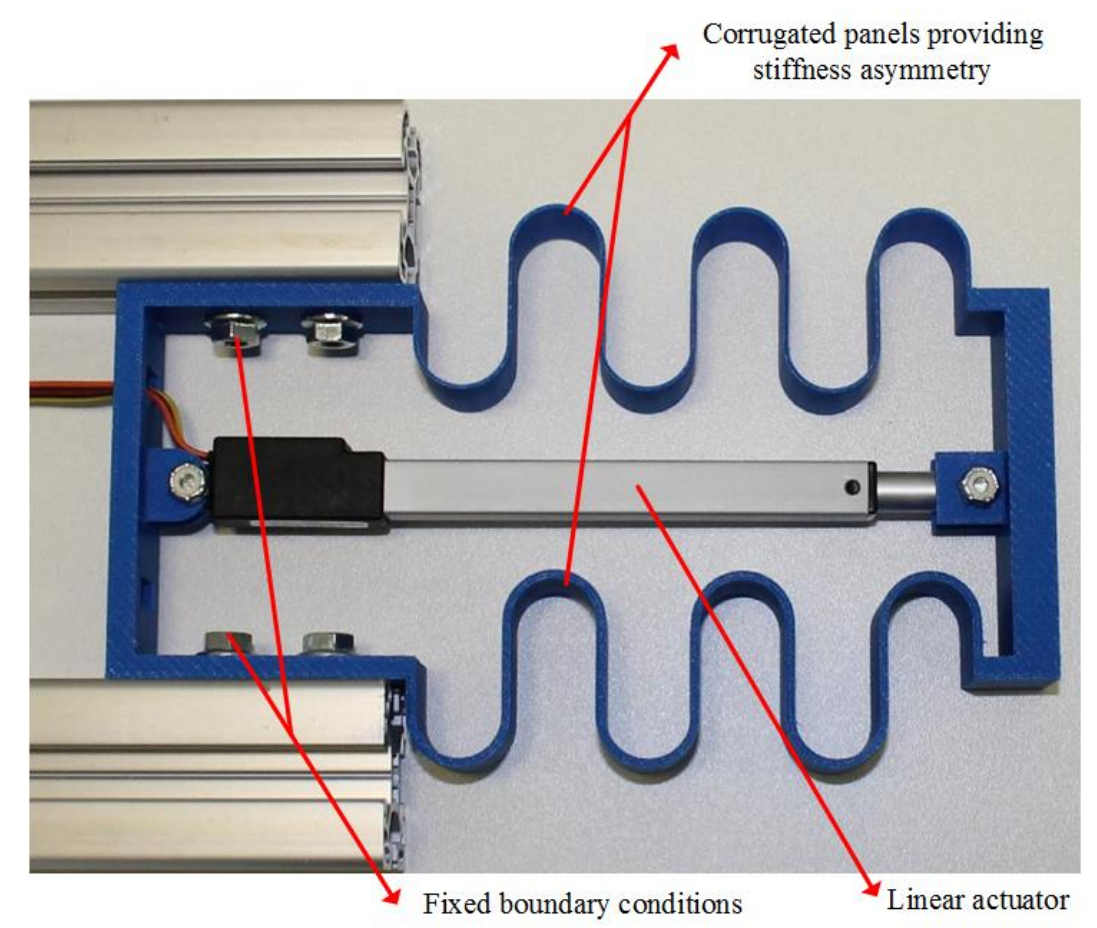

Figure 5.8 A compliant structure based on stiffness asymmetry

Samples of the compliant structures are manufactured by 3D printing. As shown in Figure 5.8, the corrugated panel is clamped by two screws to ensure the fixed boundary condition, and the compliant structure is actuated by a linear actuator.

A controller is used to control the feedback position of the actuator, and the power for the actuator is provided by batteries. The connections between the linear actuator and the structure are pinned to ensure that the linear actuation force can be provided and the rotation of the compliant structure cannot be influenced. The movement of the sample is recorded by a camera mounted perpendicular to it. Three samples are manufactured with their geometry parameters summarised in Table 5.1. Sample 1 and Sample 2 have the same geometry parameters but opposite corrugation direction. In Sample 1, the two round corrugated panels are both clamped at point A, while in Sample 2, one round corrugated panel is clamped at point A, and the other at point $\mathrm{A}^{\prime}$ (see Figure 4.2 for the geometry definition of the corrugation), which leads to the opposite corrugated direction as shown by the red arrows in Figure 5.9(a). Sample 3 has the same corrugated directions for both corrugated panels, but the thicknesses of 
the panels are not the same, which will cause a stiffness asymmetry in the compliant structure.

\begin{tabular}{|c|c|c|c|c|c|c|}
\hline Sample name & ${ }^{1} L(\mathrm{~m})$ & ${ }^{1} R(\mathrm{~m})$ & ${ }^{1} t(\mathrm{~m})$ & ${ }^{2} L(\mathrm{~m})$ & ${ }^{2} R(\mathrm{~m})$ & ${ }^{2} t(\mathrm{~m})$ \\
\hline Sample 1 & 0.01 & 0.01 & 0.002 & 0.01 & 0.01 & 0.002 \\
\hline Sample 2 & 0.01 & 0.01 & 0.002 & 0.01 & 0.01 & 0.002 \\
\hline Sample 3 & 0.01 & 0.01 & 0.001 & 0.01 & 0.01 & 0.003 \\
\hline
\end{tabular}

Table 5.1 Geometry parameters of the round corrugation samples

The deflections of the samples are measured from the digital photographs. Figure 5.9(a) shows the different deformations of the samples and Figure 5.9(b) shows the comparison of the deflections between the analytical and experimental results. The deflections are normalised to show the different slopes since the rotation of the compliant structure is one of its key features, and the slope of the curve is determined by the geometry properties, not related to the material properties and actuation force.
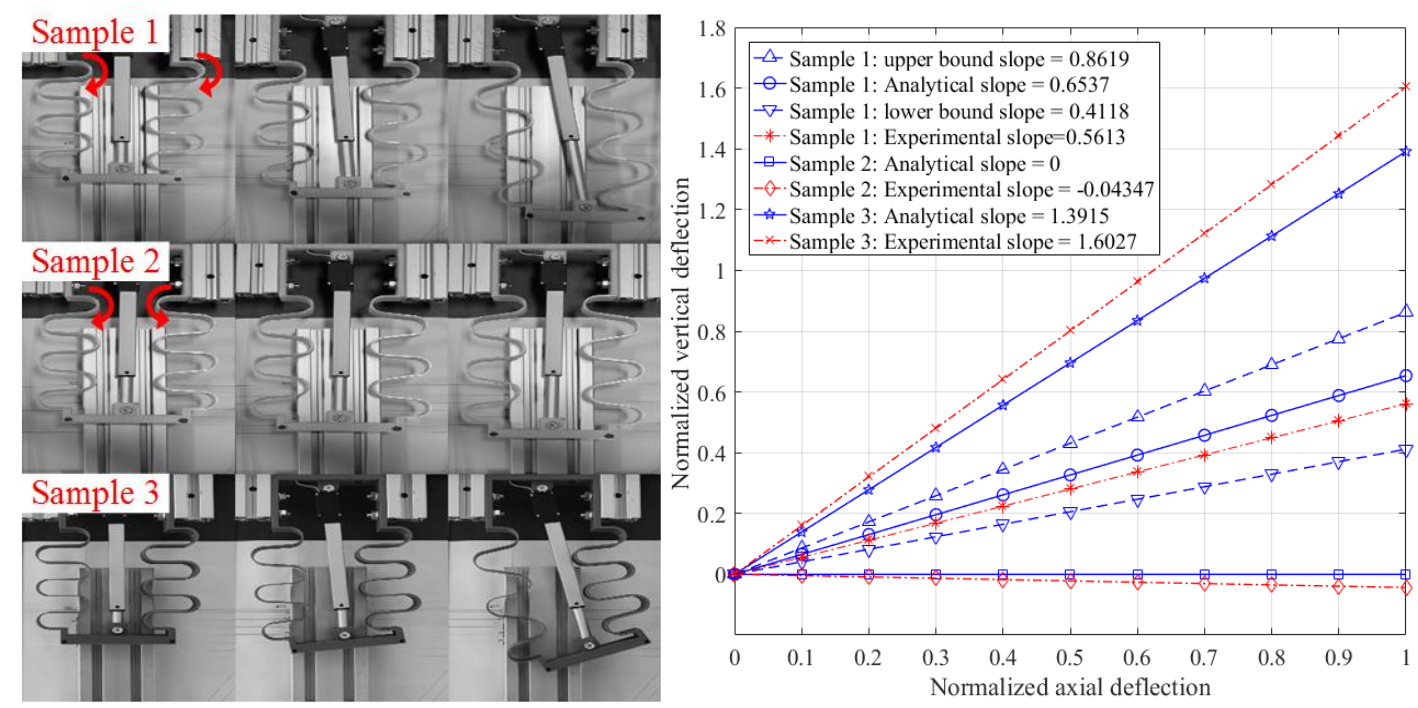

Figure 5.9 (a) Deflections of the sample under actuation; (b) Comparison of the slopes between the lateral and axial deflections

Sample 2 almost has no vertical deflection since its two corrugated panels have opposite vertical deflections, cancelling out the final rotation of the whole compliant structure. Since the two corrugated panels of Sample 1 have the same stiffness, the 
vertical deflection is only caused by the coupling effect. However, in Sample 3, a larger slope is found due to the stiffness asymmetry in the compliant structure. Although some errors exist between the analytical and experimental results, the differences are reasonable due to manufacturing tolerance.

Some errors can be found in the experiment due to many reasons, such as the error during the image processing, the friction on the samples, the inaccurate actuator location, and so on. The uncertainty caused by the manufacturing process is analysed since the samples are 3D printed, which is a relatively new manufacturing method. The resolution of the $3 \mathrm{D}$ printer used in the experiment is $0.1 \mathrm{~mm}$ [118], which means the error in the geometry parameters could be $0.1 \mathrm{~mm}$ in the samples.

For instance, the influence of the geometry uncertainty in Sample 1 due to manufacturing is shown in Figure 5.9(b). The geometry uncertainty is included in the equivalent model to obtain the upper and lower bounds of the slope of Sample 1, in which the geometry variables are within the range as follows

$$
\left\{\begin{array}{c}
{ }^{i} L-1 \% \cdot{ }^{i} L \leq{ }^{i} L \leq{ }^{i} L+1 \% \cdot{ }^{i} L \\
{ }^{i} R-1 \% \cdot{ }^{i} R \leq{ }^{i} R \leq{ }^{i} R+1 \% \cdot{ }^{i} R \\
{ }^{i} t-5 \% \cdot{ }^{i} t \leq{ }^{i} t \leq{ }^{i} t+5 \% \cdot{ }^{i} t
\end{array}\right.
$$

Here, in Sample $1,{ }^{i} L=0.01 \mathrm{~m},{ }^{i} R=0.01 \mathrm{~m},{ }^{i} t=0.002 \mathrm{~m}(i=1,2)$.

Furthermore, the geometry error caused by the printer resolution can also be found along the $z$-direction. The thickness of the corrugated panels varies from the bottom to the top of the sample, which makes the $2 \mathrm{D}$ assumption not satisfied perfectly. The detailed investigation of the geometry uncertainty and other systematic errors is beyond the scope of the thesis, while the error between the experimental and analytical slope of Sample 1 is within the range determined by Equation (5.8), which has at least verified the analytical result to some extent. 


\subsection{Parametric study of the actuation location and the height of the compliant structure}

In this chapter, the geometry variables of the corrugated panels will be optimised, while some geometry parameters are constrained by other factors, which are fixed in the optimisation. Parametric studies are performed to find the effects of the actuation location, $L_{a c t}$, and the height of the compliant structure, $b$. The influence of the length of the compliant structure is not investigated since it is determined by the size of the compliant structure, and inherently influenced by the corrugation geometry.

The geometry parameters in the test cases are listed in Table 5.2. The thickness in the upper panel $\left({ }^{1} t\right)$ is smaller than that in the lower $\left({ }^{2} t\right)$, which leads to a more flexible part with all the other corrugation geometry parameters remaining the same.

\begin{tabular}{|c|c|c|c|c|c|c|c|c|c|c|}
\hline & ${ }^{1} L(\mathrm{~m})$ & ${ }^{1} R(\mathrm{~m})$ & ${ }^{1} t(\mathrm{~m})$ & ${ }^{1} n$ & ${ }^{2} L(\mathrm{~m})$ & ${ }^{2} R(\mathrm{~m})$ & ${ }^{2} t(\mathrm{~m})$ & ${ }^{2} n$ & $b(\mathrm{~m})$ & $L_{a c t}$ \\
\hline $\begin{array}{c}\text { Test case } \\
\text { of } L_{\text {act }}\end{array}$ & 0.02 & 0.02 & 0.002 & 2 & 0.02 & 0.02 & 0.003 & 2 & 0.2 & \\
\hline $\begin{array}{c}\text { Test case } \\
\text { of } b\end{array}$ & 0.02 & 0.02 & 0.002 & 2 & 0.02 & 0.02 & 0.003 & 2 & 0.5 \\
\hline
\end{tabular}

Table 5.2 geometry parameters in the parametric analysis cases

The actuation location, $L_{a c t}$, is where the actuation force is applied in the connection beam between the two corrugated panels, which remains in the middle of the compliant structure in the above sections. Here, the location is nondimensionalized, and varies from 0 to 1 corresponding to the location of the upper and lower parts as shown in Figure 5.10(a). No aerodynamic force is applied to simplify the problem.

More beam elements are used to model the connection beam between the two corrugated panels so that the different actuation locations can be obtained. While the actuation location varies, the deflection of the compliant structure is always represented by the node in the middle of the compliant structure, which ensures the deflections can be nondimensionalized and compared. 

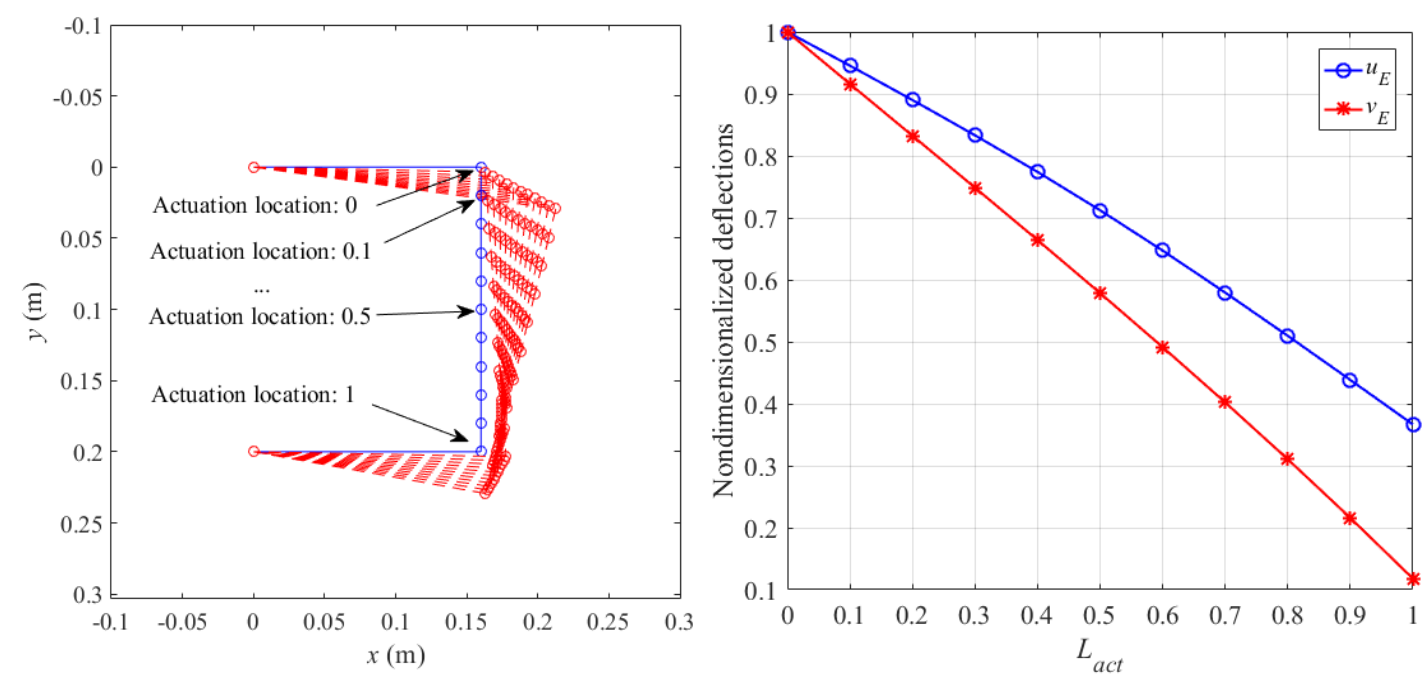

Figure 5.10 (a) Actuation location (black arrow), un-deformed structure(blue lines) and deformed structure (red lines), (b) Nondimensionalized deflections under different actuation locations

As shown in Figure 5.10(b), the maximum deflections are found with the minimum actuation location (0), which corresponds to the more flexible part in the compliant structure. The results point out the actuation force should be applied as close to the more flexible part as possible. Since more force is allocated to the more flexible part, an even larger deformation to the compliant structure could be generated. The results also indicate the actuation force applied in the middle is a conservative option, while the location is also affected by the size of the actuation system and corrugations. The subsequent analysis will apply the actuation force at the middle of the beam if not specified otherwise.

The height of the compliant structure, $b$, is the distance between the two corrugated panels. The height is constrained by the available space of the compliant structure. The deflection of the middle point is still considered, which is transferred to the rotation angle of the compliant structure. The influence of the axial deflection is taken into account when the rotation is calculated since the deflection could be relatively large compared to the length of the structure. Figure 5.11 shows the normalised rotation angle while the height $b$ is changing. The rotation of the compliant structure is reduced by the increased height under either the actuation 
force, $F$, or the aerodynamic force, $P$. The rotation angles are divided by the maximum rotation angle in the parametric study, so the rotation angle starts from 1 in Figure 5.11.

The results show the influence of the spar height in the aircraft structure. A large spar height is able to provide a significant stiffness, which can reduce the deformation under the loads.

The selection of the height $b$ is determined by the detailed design. The entire height of the compliant structure should also be obtained including the corrugation geometry. An example is introduced later in Section 6.2.

The height $b$ works as a constraint in the optimisation as represented in Section 5.4. The selection becomes very sensitive when the space available is limited. An improper constraint might lead to the failure of the optimisation. The different geometry feature of the round and trapezoidal corrugation also provides different results.
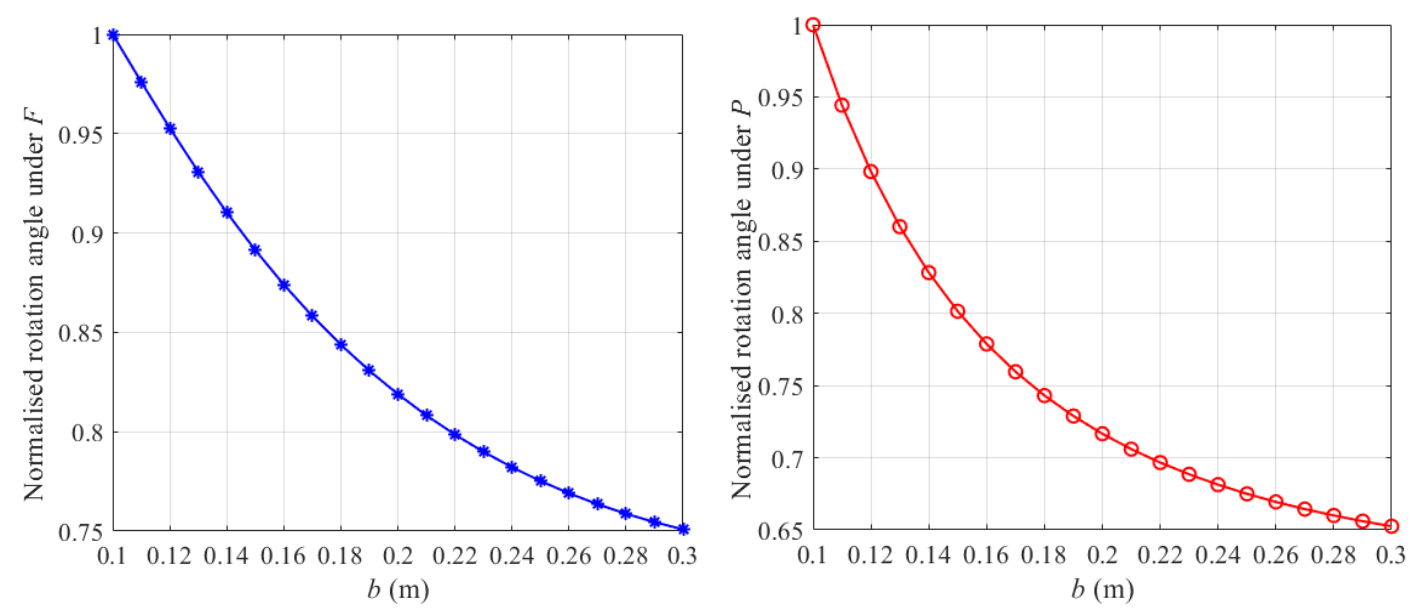

Figure 5.11 Normalised rotation angle with changed height $b$ under (a) actuation force $F$ and (b) aerodynamic force $P$ 


\subsection{Optimisation of a single compliant structure}

\subsubsection{Optimisation setup}

To achieve a larger deformation, multiple units of the compliant structure can be used together. As explained in Section 5.2.2 and Figure 5.12, the superscript ' $j$ ' means the unit number. Each compliant structure unit is under the actuation force $F^{j}$ and aerodynamic force $P^{i}$. Since the compliant structure is installed in the wing structure, the height of the structure, $b$, is determined by the thickness of the airfoil, which is assumed to be a fixed parameter in the optimisation.

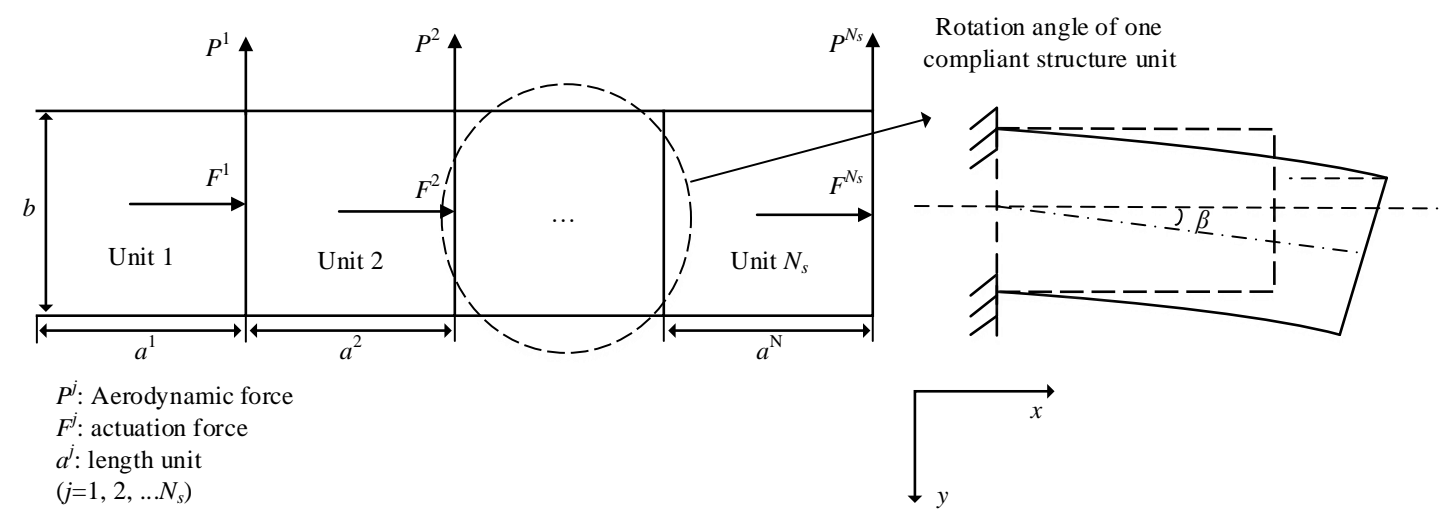

Figure 5.12 Multiple units of compliant structures

The optimisation is performed using the Matlab Global Optimization Toolbox [105] and a genetic algorithm. The optimisation is first conducted for a single unit of compliant structure, in which the length $a^{j}$, height $b^{j}$, width $w^{j}$ and aerodynamic force $P^{j}$, are all fixed parameters. It should be noted that the corrugated panel in each compliant structure unit can also consist of multiple (round or trapezoidal) corrugation units, where the number of corrugations is denoted by ' $n$ ', while the number of the compliant structure units is denoted by ' $N_{s}$ '.

The shape change of the compliant structure is represented by the rotation angle $\beta$ as shown in Figure 5.12, which is provided by external sources and fixed during one optimisation. The aerodynamic force $P^{j}$ is applied to each unit of the structure, which is also provided by external sources. By doing so, a single objective optimisation of the compliant structure can find the optimal geometry variables of the compliant 
structure, which is capable of carrying aerodynamic loads and shape-changing simultaneously, and the requirement of the actuation force is minimised, which will reduce the weight, and other difficulties of the actuation system.
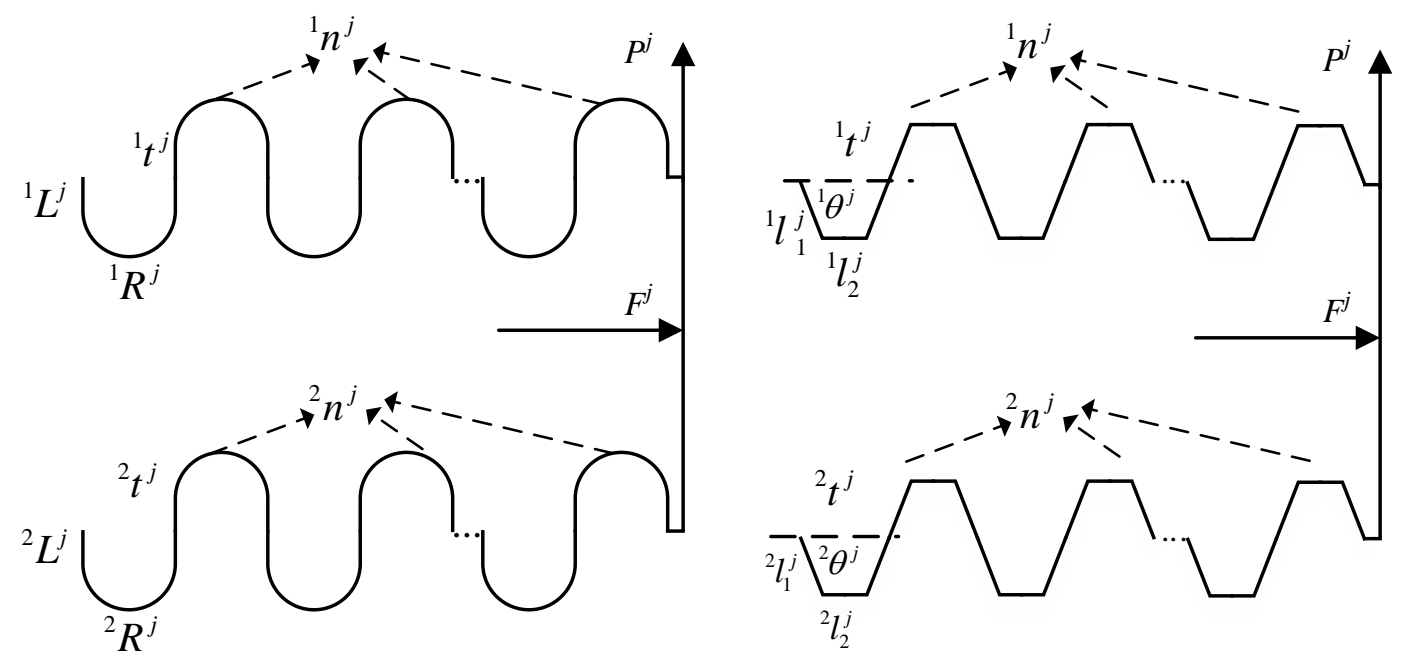

Figure 5.13 Optimisation variables of compliant structures made of on (a) round, (b) trapezoidal corrugated panels

Round corrugated panels are optimised first in this chapter, and the variables of the round corrugated panels are shown in Figure 5.13(a). The number of corrugations, ${ }^{1,2} n$, should be integers. The bounds of the variables are shown below for the case study. The genetic algorithm has 200 individuals and 600 generations in this case. Since only one compliant structure is optimised, the superscript $j$ is neglected. The length, $a$, height, $b$, and width, $w$, of the compliant structure is $0.1 \mathrm{~m}, 0.1 \mathrm{~m}$ and $0.1 \mathrm{~m}$ respectively. The objective is to minimise the actuation force $F$ for the required rotation angle as

$$
\min F
$$

The variables are in the ranges

$$
\left\{\begin{array}{l}
{ }^{1,2} t\left(0.001 \leq{ }^{1,2} t \leq 0.02 \mathrm{~m}\right) \\
{ }^{1,2} L\left(0.01 \leq{ }^{1,2} L \leq 0.05 \mathrm{~m}\right) \\
{ }^{1,2} n\left(1 \leq{ }^{1,2} n \leq 4, n=1,2 \ldots\right)
\end{array}\right.
$$


Geometry constraints are applied to the compliant structure to provide a realistic geometry and enough space to install the actuation system. A structural constraint is added to control the maximum von Mises stress in the corrugated panel. And the rotation angle of the compliant structure should be equal to the required rotation angle of this unit. Thus the constraints of the optimisation are given as

$$
\begin{aligned}
& { }^{1} L+{ }^{1} R+{ }^{2} L+{ }^{2} R<b / 2 \\
& { }^{i} t<{ }^{i} L / 5(i=1,2) \\
& { }^{i} t<{ }^{i} R / 5(i=1,2) \\
& \max \sigma_{v} \leq \sigma_{y} \\
& \beta=0,2^{\circ}, 4^{\circ}, \ldots 8^{\circ}
\end{aligned}
$$

where the round corrugation radius ${ }^{i} R$ is determined by the length of the structure $a$ and the number of corrugations as ${ }^{i} R=a /(4 n)(i=1,2)$. The von Mises stress and the yield stress of the material are denoted as $\sigma_{v}$ and $\sigma_{y}$ respectively. The von Mises stress in the corrugated panel is obtained by calculating the nodal loads in the equivalent beam element after the nodal displacements of the structure are obtained. The yield stress is 270MPa, the material's Young's modulus is $72 \mathrm{GPa}$, and the Poisson's ratio is 0.3 in this study. The required rotation angle of compliant structure varies from 0 to $8^{\circ}$.

\subsubsection{Influence of the required shape change and aerodynamic loads}

Figure 5.14(a) shows the optimised actuation force when the required rotation angle varies from 0 to $10^{\circ}$ with different aerodynamic forces. Clearly, a larger actuation force will be required if the required angle or the aerodynamic force is increased.

When the required rotation angle is 0 , which means the compliant structure is only supposed to carry the aerodynamic loads and maintain the geometry, the optimised variables are different to those when the compliant structure is supposed to change its shape (the required rotation angle larger than 0 ). The optimised variables are summarised in Table 5.3. The different optimised variables to maintain geometry and to deform the structure indicate the influence of the deformation of the structure. 

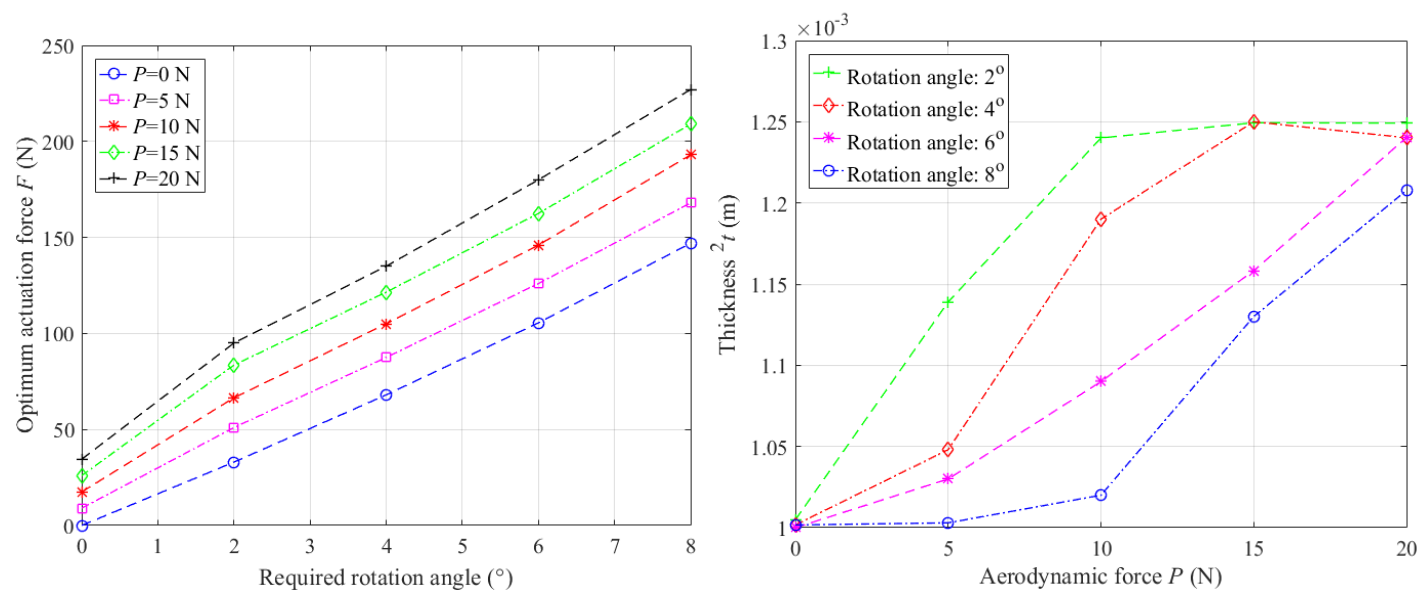

Figure 5.14 (a) Optimised actuation force vs required rotation angle, (b) Optimised thickness ${ }^{2} t$ vs aerodynamic force

While the other variables are fixed, the trend of the panel thickness in the lower part ${ }^{2} t$ represents the change of total stiffness and stiffness asymmetry in the compliant structure. As shown in Figure 5.14(b), the thickness ${ }^{2} t$ panel is influenced by the required rotation angle and the aerodynamic force.

According to Equation (4.33), the equivalent extension stiffness will be determined by the geometry parameter and the material's Young's modulus $E$. When the aerodynamic force increases, the thickness ${ }^{2} t$ is increased to provide a relatively large total stiffness of the structure since the other parameters are fixed. The stiffness asymmetry of the structure is also increased, which can help to increase the rotation angle, or reduce the actuation force if the rotation angle is specified. Although the numbers of corrugations are at their upper bound, providing the smallest $R$ and increasing the extension stiffnesses of the corrugated panels, the thickness ${ }^{1} t$ is at its lowest bound, which provides the smallest extension stiffness to the corrugated panel.

A compromise has been made by the optimised variables to satisfy the constraints and to determine an optimum stiffness allocation in the structure. With the trade-off design, the total stiffness can be large enough to carry the load $P$ due to the smallest $R$, while large stiffness asymmetry can also be induced due to the different $L$ and $t$ in the two corrugated panels. 


\begin{tabular}{|c|c|c|c|c|c|c|}
\hline Required rotation angle $\left({ }^{\circ}\right)$ & ${ }^{1} L(\mathrm{~m})$ & ${ }^{1} n$ & ${ }^{1} t(\mathrm{~m})$ & ${ }^{2} L(\mathrm{~m})$ & ${ }^{2} n$ & ${ }^{2} t(\mathrm{~m})$ \\
\hline 0 & 0.0186 & 4 & 0.001 & 0.0125 & 2 & 0.0025 \\
\hline $2,4,6,8$ & 0.0275 & 4 & 0.001 & 0.01 & 4 & \\
\hline
\end{tabular}

Table 5.3 Optimised variables of the compliant structure. Note that the optimised value ${ }^{2} t$ varies, as shown in Figure 5.14(b)

\subsubsection{Influence of the geometry constraint}

Further optimisation cases are performed to find the influence of the height $b$. Equation (5.10) is used to prevent any interference of the corrugations, while it also affects the optimal results. The smallest $R$ has been found in Section 5.4.2, which would also help to meet the constraint. Figure 5.15 shows the influence of the height $b$ on the actuation force and the geometry variables. The required rotation angle is $6^{\circ}$ and the aerodynamic force $P$ is $5 \mathrm{~N}$ in this test.

The optimised actuation force is reduced when the height increases, which seems different to the result in Section 5.3: the rotation angle is reduced when the height increases. The smaller actuation force is caused by the smaller total stiffness of the compliant structure, which is determined by the optimised geometry variables. The optimised geometry variables are listed in Table 5.4. The variables are fixed at their lower bounds except for the variable ${ }^{1} L$, which increases with the height $b$.
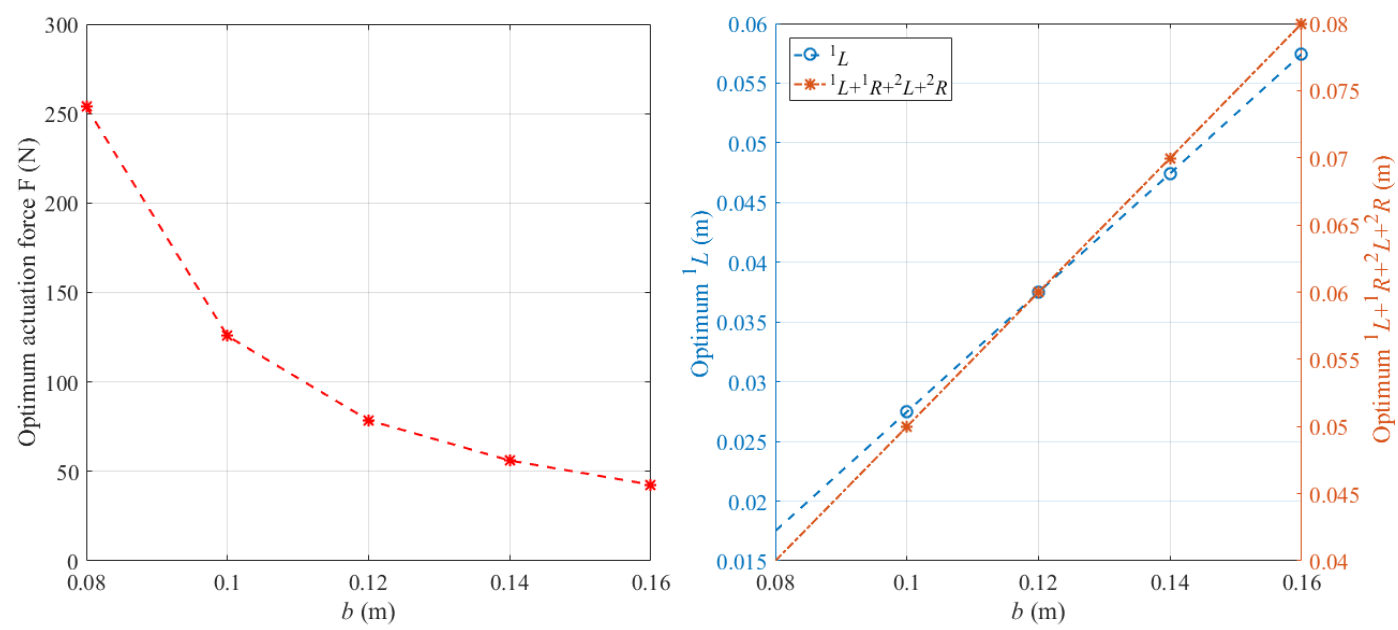

Figure 5.15 Influence of height b on (a) optimised actuation $F$, (b) geometry variable

${ }^{1} L$ and the sum of ${ }^{1} L+{ }^{1} R+{ }^{2} L+{ }^{2} R$ 


\begin{tabular}{|c|c|c|c|c|c|}
\hline${ }^{1} L(\mathrm{~m})$ & ${ }^{1} R(\mathrm{~m})$ & ${ }^{1} t(\mathrm{~m})$ & ${ }^{2} L(\mathrm{~m})$ & ${ }^{2} R(\mathrm{~m})$ & ${ }^{2} t(\mathrm{~m})$ \\
\hline & 0.0063 & 0.001 & 0.01 & 0.0063 & 0.001 \\
\hline
\end{tabular}

Table 5.4 Optimised geometry variables when the height $b$ varies. Note that the optimised value ${ }^{1} L$ varies, as shown in Figure 5.15(b)

As shown in Figure 5.15(b), the increase of the variable ${ }^{1} L$ makes the sum of the ${ }^{1} L+{ }^{1} R+{ }^{2} L+{ }^{2} R$ follow the increased height $b$, and remain at the maximum possible value $(b / 2)$. The thickness of the lower corrugated panel ${ }^{2} t$ does not contribute to the unsymmetrical stiffness but is kept at the lower bound to reduce the actuation force. It can be concluded when the required rotation and aerodynamic force is constant, the optimised ${ }^{1} L$ provides the main stiffness asymmetry in the compliant structure. Considering the change of ${ }^{1} L$ in Section 5.4.2, its importance to induce the stiffness asymmetry was highlighted.

However, the optimisation of round corrugated panels becomes unavailable if the height is too small. In this test, no optimum results can be found when the height $b$ is reduced to $0.06 \mathrm{~m}$ due to the constraints.

Since both the variables $L$ and $R$ contribute to the height constraint, it seems difficult for the round corrugation to satisfy a very small height constraint. On the other side, the trapezoidal corrugation might be able to satisfy a small height constraint because the inclined angle of the trapezoid could be very small, which leads to a small contribution to the height.

The optimisation variables of the complaint structure using trapezoidal corrugated panels are shown in Figure 5.13(b). The trapezoidal corrugation is then tested with a small height $b$. The bounds of the variables are shown below for the case study. The optimisation setup remains the same as that for the round corrugated panels. The objective is to minimise the actuation force as

$$
\min F
$$

The range of the variables are 


$$
\left\{\begin{array}{l}
{ }^{1,2} t\left(0.001 \leq{ }^{1,2} t \leq 0.005 \mathrm{~m}\right) \\
{ }^{1,2} l_{1}\left(0.01 \leq{ }^{1,2} l_{1} \leq 0.025 \mathrm{~m}\right) \\
{ }^{1,2} \theta\left(5 \leq \theta \leq 90^{\circ}\right) \\
{ }^{1,2} n\left(1 \leq{ }^{1,2} n \leq 4, n=1,2 \ldots\right)
\end{array}\right.
$$

The constraints are

$$
\left\{\begin{array}{c}
{ }^{1} l_{1} \sin { }^{1} \theta+{ }^{2} l_{1} \sin ^{2} \theta \leq b / 2 \\
{ }^{1} t \leq \frac{1}{5}{ }^{1} l_{1} ; \quad{ }^{1} t \leq \frac{1}{5}{ }^{1} l_{2} \\
{ }^{2} t \leq \frac{1}{5}{ }^{2} l_{1} ; \quad{ }^{2} t \leq \frac{1}{5}{ }^{2} l_{2} \\
2{ }^{1} l_{1} \cos { }^{1} \theta \leq \frac{a}{2{ }^{1} n} \\
2{ }^{2} l_{1} \cos { }^{2} \theta \leq \frac{a}{2^{2} n} \\
\max \sigma_{v} \leq \sigma_{y} \\
\beta=6^{\circ}
\end{array}\right.
$$

The optimisation results are summarised in Tables 5.5 and 5.6, which do satisfy the geometry constraints.

\begin{tabular}{|c|c|c|c|c|c|c|}
\hline$b(\mathrm{~m})$ & $F(\mathrm{~N})$ & ${ }^{1} l_{1}(\mathrm{~m})$ & ${ }^{1} \theta\left({ }^{\circ}\right)$ & ${ }^{1} n$ & ${ }^{1} t(\mathrm{~m})$ & ${ }^{1} l_{1} \sin { }^{1} \theta+{ }^{2} l_{1} \sin ^{2} \theta(\mathrm{m})$ \\
\hline 0.04 & 230.2 & 0.019 & 89.99 & 4 & 0.001 & 0.02 \\
\hline 0.05 & 171.9 & 0.024 & 89.99 & 4 & 0.001 & 0.025 \\
\hline 0.06 & 151.3 & 0.025 & 89.99 & 4 & 0.001 & 0.03 \\
\hline
\end{tabular}

Table 5.5 Optimisation results of the upper panel with changing height $b$

\begin{tabular}{|c|c|c|c|c|c|c|}
\hline$b(\mathrm{~m})$ & $F(\mathrm{~N})$ & ${ }^{2} l_{1}(\mathrm{~m})$ & ${ }^{2} \theta\left({ }^{\circ}\right)$ & ${ }^{2} n$ & ${ }^{2} t(\mathrm{~m})$ & ${ }^{1} l_{1} \sin { }^{1} \theta+{ }^{2} l_{1} \sin { }^{2} \theta(\mathrm{m})$ \\
\hline 0.04 & 230.2 & 0.01 & 5.02 & 4 & 0.001 & 0.02 \\
\hline 0.05 & 171.9 & 0.01 & 5.03 & 4 & 0.001 & 0.025 \\
\hline 0.06 & 151.3 & 0.01 & 29.62 & 4 & 0.001 & 0.03 \\
\hline
\end{tabular}

Table 5.6 Optimisation results of the lower panel with changing height $b$

From Tables 5.5 and 5.6, it can be concluded that compliant structures consisting of 
trapezoidal corrugated panels can satisfy small height constraints, which cannot be met by round corrugation panels. With the decreased height, the optimum variable ${ }^{1} l_{1}$ and the inclined angle ${ }^{2} \theta$ are reduced to satisfy the height constraint, while the other optimum variables remain constant, which keeps the combination of the ${ }^{1} l_{1}+$ ${ }^{1} \theta+{ }^{2} l_{1}+{ }^{2} \theta$ at the upper limit of the constraint.

\subsubsection{Available maximum shape change}

The optimisation is also performed to find the maximum rotation angle $\beta$ that the compliant structure can achieve. The objective becomes the maximum rotation angle and the actuation force is increased continuously in the optimisation until the maximum von Mises stress in the compliant structure reaches the yield stress. The yield stress is kept at $270 \mathrm{MPa}$ in the optimisation, and compliant structures consisting of round or trapezoidal corrugated panels are both tested. The aerodynamic force is 0 to represent the ground test condition. The length, $a$, height, $b$, and width, $w$, of the compliant structure is $0.1 \mathrm{~m}, 0.1 \mathrm{~m}$ and $0.1 \mathrm{~m}$ respectively.

The results are listed in Table 5.7. The round and trapezoidal corrugated panels can provide a rotation angle over $10^{\circ}$. The required actuation force using the trapezoidal corrugated panels is larger than for the round corrugations.

\begin{tabular}{|c|c|c|}
\hline & Maximum $\beta\left(^{\circ}\right)$ & Required $F(\mathrm{~N})$ \\
\hline Round corrugation & 12.13 & 246.6 \\
\hline Trapezoidal corrugation & 13.16 & 336.0 \\
\hline
\end{tabular}

Table 5.7 Maximum rotation angle of the compliant structure

\subsection{Optimisation of multiple units of compliant structures}

The optimisation of a single compliant structure has highlighted its features, while the maximum rotation is restricted by the material. Multiple units of compliant structures can be employed together to achieve a more substantial shape change. 
Since the optimisation of one single compliant structure requires six variables, it is necessary to decompose the optimisation of the multiple units of compliant structures to sub-problems for each of the compliant structures.
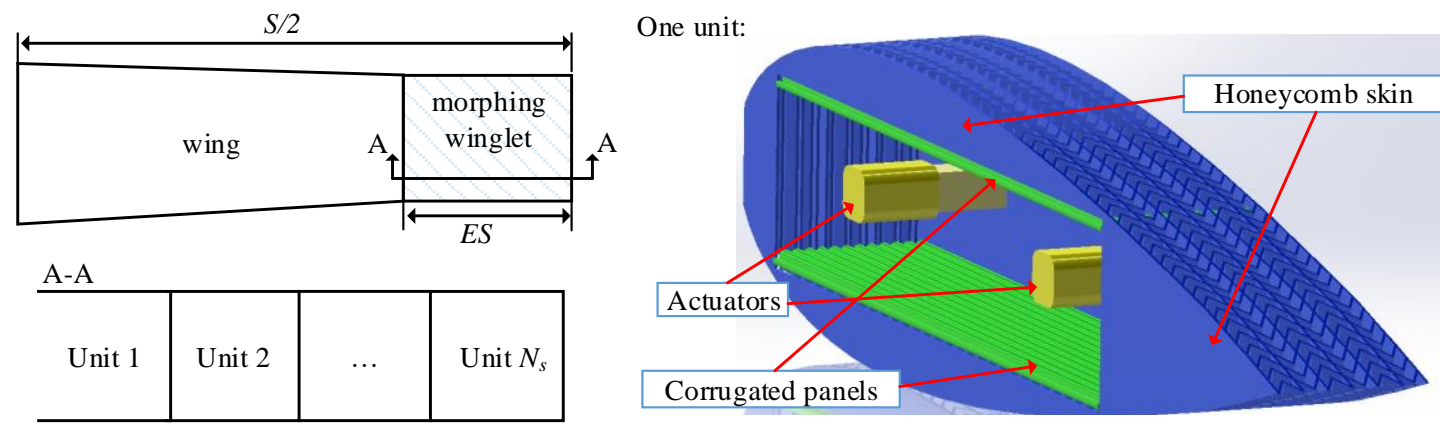

Figure 5.16 (a) Wing span extension via the morphing winglet, (b) Conceptual design of the morphing winglet

As shown in Figure 5.16(a), the multiple units of the compliant structure are installed to extend the wing span of a baseline design. In this case, the length of each compliant structure is identical. Thus, the entire length of the extension will be $E S=N_{s} \cdot a$. The extended length $E S$ is determined by the wing structure and flight conditions. It is obvious that a larger ES will cause a larger possible deformation, which could lead to more morphing benefits. However, the extension of the wing span is constrained by the wing root bending moment and flutter performance of the aircraft due to the increased weight at the wing tip. Figure 5.16(b) shows a conceptual design of the compliant structure, illustrating the corrugated panels and actuators, as well as a solution to maintain the aerodynamic shape using a flexible honeycomb skin. The flexible honeycomb structure has been applied in the span-extension morphing wing $[38,119]$. In the literature, the honeycomb structure is used to support the elastomeric matrix composite to achieve a high out-of-plane stiffness, while in the current study the aerodynamic loads are assumed to be carried by the corrugated panels. The honeycomb skin will be tailored to be more flexible than the corrugated panels, and will only carry local loads and maintain the geometry. The proposed solution indicates that the optimisation of the corrugated panels will be sufficient to provide reasonable estimate of the performance benefits. 
As shown in Figure 5.17, a simple two-level optimisation procedure is applied to find the optimal designs of each unit. The entire length of the compliant structures $E S$ is assumed to be fixed. The global variable in the first level is the number of compliant structure units, $N_{s}$. Another external input of the optimisation procedure is the distribution of the aerodynamic loads on the wing and the retrofitted wing tip, which will be used to obtain the concentrated forces $F^{j}(j=1,2, \ldots)$ on each compliant structure. If the required rotation angle of each compliant structure is also known, the optimisation of each compliant structure is then performed in sequence, which can be used to size the actuation system, and estimate the associated weight increase.

The range of the variables are based on the geometry parameters in each compliant structure unit as

$$
\left\{\begin{array}{c}
{ }^{1,2} t^{j}\left(0.001 \leq^{1,2} t^{j} \leq 0.02 \mathrm{~m}\right) \\
{ }^{1,2} L^{j}\left(0.01 \leq^{1,2} L^{j} \leq b^{j} / 2 \mathrm{~m}\right) \\
{ }^{1,2} n^{j}\left(1{ }^{1,2} n^{j} \leq a^{j} / 4\left(\min R^{j}\right), n=1,2 \ldots\right) \min R^{j}=0.01 \mathrm{~m}
\end{array}\right.
$$

The constraints are similar to those in the single unit optimisation as

$$
\left\{\begin{array}{l}
{ }^{1} L+{ }^{1} R+{ }^{2} L+{ }^{2} R<b / 2 \\
{ }^{i} t<{ }^{i} L / 5(i=1,2) \\
{ }^{i} t<{ }^{i} R / 5(i=1,2) \\
\max \sigma_{v} \leq \sigma_{y} \\
\beta=6^{\circ}
\end{array}\right.
$$




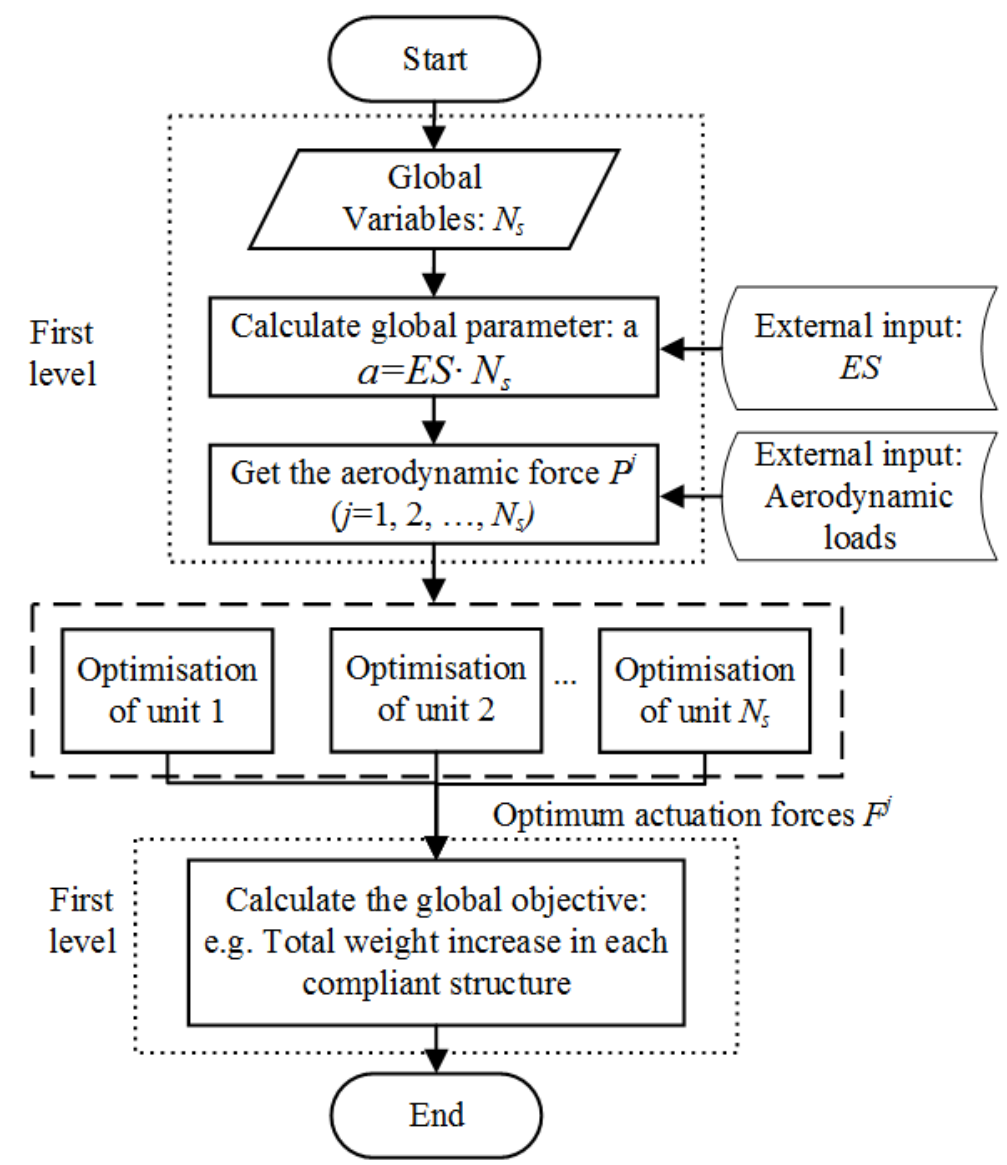

Figure 5.17 Optimisation procedure of the multiple units of the compliant structure

In the current study, two optimisation cases are considered. First, a hypothetical case is tested, in which the wing span, $S$, is $6 \mathrm{~m}$, and the extended length, $E S$, is fixed at $0.5 \mathrm{~m}$. The aerodynamic load distribution is assumed to be perfectly elliptical and the total lift is $1000 \mathrm{~N}$. The height and the width of each compliant structure is $b=0.1 \mathrm{~m}$, and $w=0.1 \mathrm{~m}$ respectively. In this case, the objective is to minimise the sum of the actuation forces in all the compliant structure units as

$$
\min \sum_{1}^{N_{s}} F^{j}
$$

Figure 5.18(a) shows that as the number of complaint structures increases, the sum of the optimised actuation forces is not monotonic, which means that for a fixed span extension, a trade-off selection of the length of each compliant structure should be made to reduce the total actuation force. 
The assumption of a fixed span extension leads to fixed aerodynamic loads, which simplifies the optimisation problem. In contrast, a changing wing span will require both structural and aerodynamic analysis, and hence another optimisation case is performed in a more realistic situation as proposed in Chapter 3. In this case, the optimistion is peformed in sequence to minimise the actuation force in each compliant structure unit as

$$
\min F^{j}
$$

The compliant structure is used to change the dihedral angle of the morphing winglet in the regional airliner. The length and height of a compliant structure unit are fixed at $0.1 \mathrm{~m}$ and $0.101 \mathrm{~m}$ respectively based on the wing geometry, while the span of the wing changes with the increase of the number of compliant structures. The width of the compliant structure is assumed to be equal to the wing tip chord, which is overestimated due to the leading and trailing edge. The aerodynamic loads are still obtained using the software AVL [103].

While the result in Chapter 3 has shown that the relationship between the number of compliant structures and the corresponding performance change, the stiffness asymmetry in that case study is fixed and not optimal. The potential performance improvement of morphing winglets is compromised by the weight increase of the retrofitted structure and actuation systems. The actuation force of the compliant structure is provided by a worm rack system, and the weight of the actuation system is estimated based on the specified actuation force.

In Chapter 3, the required actuation force is used to estimate the weight due to the retrofitted winglet while the stiffness asymmetry is not optimised, which overestimates the actuation force requirements. By using the optimisation, the actuation force can then be reduced, which eventually reduces the weight increase of the gross weight due to the retrofitted winglet. 

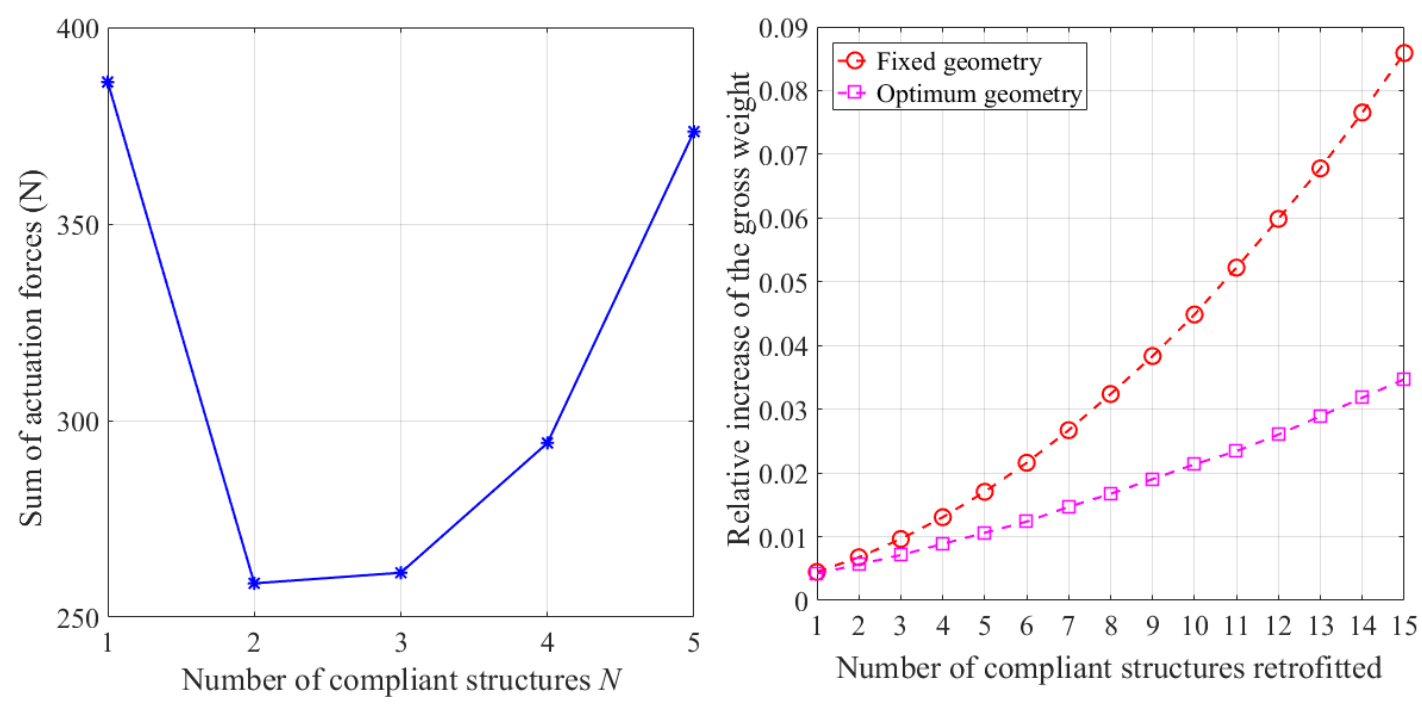

Figure 5.18 (a) Sum of the optimised actuation forces with a fixed length $E S$, (b)

Optimised weight increase compared to the results a fixed geometry

Figure 5.18(b) shows the relative increase of the gross weight before and after the actuation forces are optimised. In this case, the regional airliner is trimmed at steady level flight when it has the maximum gross weight, and the required rotation angle of each compliant structure unit is $6^{\circ}$. The objective is to minimise the actuation force in each compliant structure unit. Compared to the previous result, a significantly smaller weight increase can be obtained after the optimisation procedure is applied, which will make the proposed morphing structure more beneficial.

For instance, the range of the aircraft in steady flight is calculated using Equation (2.2). With the morphing winglet retrofitted, the range can be increased due to the larger lift to drag ratio, which will be partially compromised by the increased weight. For example, if eight units of the compliant structure are retrofitted due to other constraints, the optimisation can improve the range increase by $1.34 \%$ to $2.66 \%$.

\subsection{Summary}

In this chapter, the compliant structure proposed in Chapter 3 is optimised to explore the design space of the stiffness asymmetry in the structure and increase the benefits introduced by the structure. 
The stiffness asymmetry is provided by different geometry variables of the corrugated panels. The stiffness matrix of the compliant structure is built using the equivalent model from Chapter 4, which is verified by detailed finite element analysis and experimental tests. Three samples were manufactured, and the deformation of the samples was measured from photographs of the deformed structure. The slopes of the lateral and axial deflection are compared between the experimental and analytical results. Analysis of the geometry uncertainty due to the manufacturing process also indicates that the difference between the experimental and analytical results is acceptable. The verification also makes the optimisation of the compliant structure reasonable.

Two fixed parameters of the compliant structure are parametrically studied before an optimisation procedure is applied to find the optimised variables of the compliant structure. The optimisation of a single compliant structure shows the optimised variables are compromised to provide a stiffness asymmetry and satisfy the constraints simultaneously. The height constraint will significantly influence the optimum results and the compliant structure consisting of trapezoidal corrugated panels can satisfy a smaller height constraint than that consisting of round corrugation panels in the current optimisation setup. Also, with the current setup, a maximum rotation angle over $10^{\circ}$ can be obtained.

The optimisation is also performed for multiple units of compliant structure used as a morphing winglet. The relationship between the number of units and the objective is found when the length of the entire compliant structure is fixed. A more realistic optimisation case is conducted to obtain the optimised actuation forces for the morphing winglet, which is assumed to be retrofitted to a regional airliner. An improvement is found since the optimisation reduces the actuation forces, which reduces the weight increase due to the retrofitted morphing winglet, and improves the potential benefits brought by the morphing winglet. Although only the corrugated panels are optimised, the optimisation procedure is still able to give reasonable estimation and improve the overall performance. 


\section{Chapter 6 Design and test of a demonstration model}

\subsection{Introduction}

From Chapter 1 to Chapter 5, the conceptual design, fundamental principle, and optimisation of a morphing winglet have been illustrated. Although the concept is still far away from practical application in industry, a demonstration model of the morphing wingtip concept has been designed, manufactured and tested to highlight its potential and build a solid foundation for its further development.

Since the model is mainly for functional demonstration, the NACA 0024 airfoil with a chord $0.25 \mathrm{~m}$ is used, from consideration of the manufacturing cost and convenience. The spanwise length of the structure, $a$, is $0.12 \mathrm{~m}$. The structure of the morphing winglet consists of three main components: the leading edge, the compliant structure and the trailing edge, as shown in Figure 6.1.

The compliant structure based on the unsymmetrical stiffness has to carry the aerodynamic loads on the winglet, while the airfoil shape needs to be maintained, especially at the leading and trailing edges. In the current study, flexible honeycomb structures are used to provide and maintain the aerodynamic profile of the leading and trailing edges. The zero Poisson's ratio honeycomb design is employed due to its flexibility. Flexible honeycomb structures have been proposed and investigated in the field of morphing aircraft as solutions for spanwise or chordwise morphing [38, 39, 119, 120]. The honeycomb structures are connected to the corrugated panels evenly in the spanwise direction, which ensures the deformation caused by the actuator can spread to the whole model. A detailed FEM model is created in Abaqus ${ }^{\circledR}$ to identify possible problems in the structure in the three-dimensional(3D) cases, and the deflection results are used to estimate the shape change of the wingtip. 
In this demonstration model, an electrical linear actuator is used. The actuator is pinned at both ends so that it can rotate with the structure. Part of the upper panel in the middle is removed to install the actuator and avoid interference. An elastomer skin made of silicone rubber is used to provide the aerodynamic surface. A fairing is also added to provide an aerodynamic surface at the tip of the compliant structure.

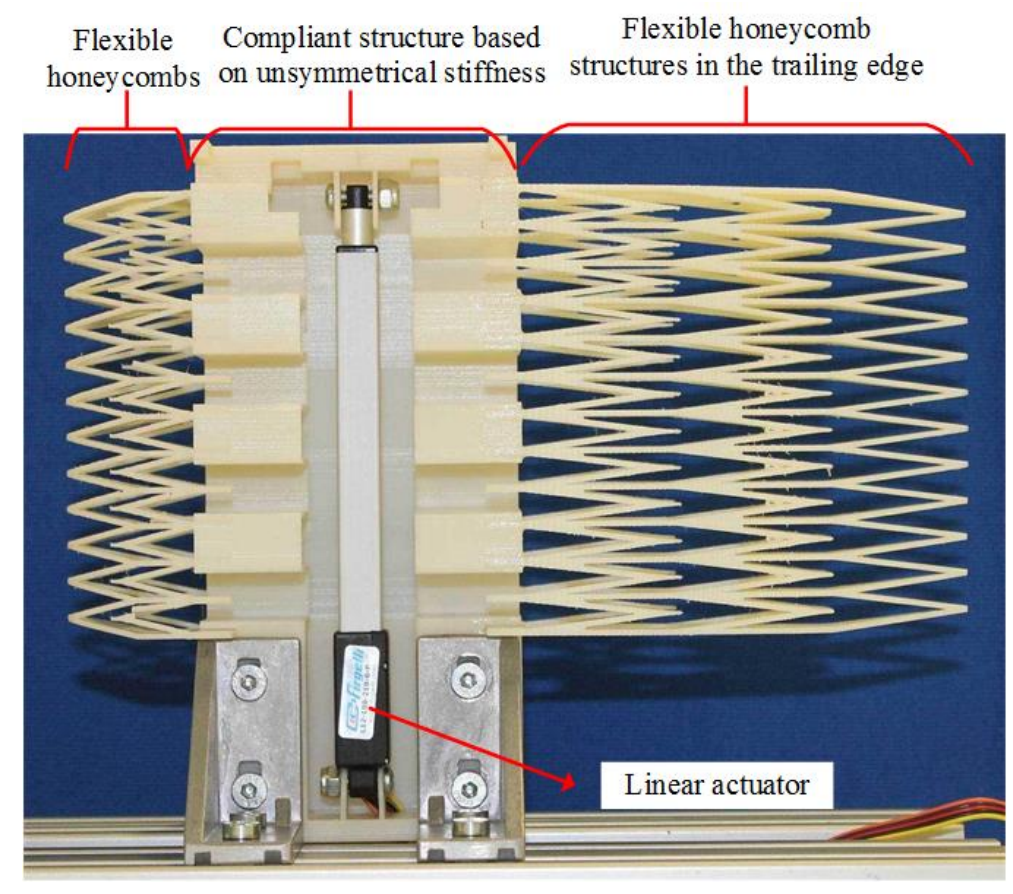

Figure 6.1 The morphing winglet demonstrator (The wingtip fairing and elastomer skin are not included)

The trapezoidal corrugated panel is investigated for the compliant structure, which is optimised to minimise the required actuation force, thus reducing the weight of the actuation system. The equivalent model of the trapezoidal corrugated panel is applied in the optimisation to reduce the calculation time as shown in Chapter 5. Although there has been extensive research on flexible honeycomb structures and corrugated structures, it is less common to combine the two types of flexible structures into one morphing aircraft design. Since the actuation force is applied to the compliant structures based on the corrugated panels, it is essential to ensure the deformation spreads evenly to the flexible honeycomb structure.

The morphing winglet is manufactured using $3 \mathrm{D}$ printing, and a static test is 
performed to demonstrate the deflection of the morphing winglet. Wind tunnel tests are also conducted to demonstrate the change of the aerodynamic performance caused by the morphing wingtip model.

The research activities in this chapter are indispensable for the further development of the morphing winglets proposed in this thesis, which can also validate the concept by the experiments. The process to overcome the problems in the detailed design of the functional model will also provide a valuable experience regarding the study of the compliant structure.

\subsection{Structure integration of the compliant structure into the winglet by optimisation}

\subsubsection{Fitting in the thickness of the airfoil}

The compliant structure based on unsymmetrical stiffness works as the primary component to carry the aerodynamic loads with the help of the linear actuator, while flexible honeycomb structures work as secondary components in the leading and trailing edge to maintain the airfoil shape. The structural integration is focused on the compliant structure based on the unsymmetrical stiffness, which is also the main body of the thesis.

The design of the corrugated panels in the compliant structure is determined by the optimisation. By employing the equivalent model proposed in Chapter 5, the calculation time for the compliant structure is reduced significantly, which leads to a faster optimisation. The compliant structure based on the unsymmetrical stiffness is optimised to reduce the actuation force, while the entire height of the compliant structure, $B\left(B=b+{ }^{1} l_{1} \sin ^{1} \theta+{ }^{2} l_{2} \sin ^{2} \theta\right)$, should be constrained to fit within the thickness of the airfoil. A sequence of optimisation is performed to find the fittest geometry variables. The variables have the same definition to those in Section 4.1 with the subscript and superscript definition defined in Section 5.2.

The objective is to minimise the actuation force as 
The ranges of the variables for the optimisation are

$$
\left\{\begin{array}{l}
{ }^{1,2} l_{1}\left(0.01 \leq{ }^{1,2} l_{1} \leq 0.025 \mathrm{~m}\right) \\
{ }^{1,2} \theta\left(0 \leq{ }^{1,2} \theta \leq 90^{\circ}\right) \\
{ }^{1,2} n\left(1 \leq{ }^{1,2} n \leq 4,{ }^{1,2} n=1,2 \ldots\right) \\
{ }^{1,2} t\left(0.0015 \leq{ }^{1,2} t \leq 0.005 \mathrm{~m}\right)
\end{array}\right.
$$

The variables need to satisfy the geometry constraints, which are

$$
\left\{\begin{array}{c}
\frac{1}{2} h_{\text {act }} f_{\text {safety }} \leq b \cdot L_{\text {act }}-{ }^{1} l_{1} \sin { }^{1} \theta \\
\frac{1}{2} h_{\text {act }} f_{\text {safety }} \leq b\left(1-L_{a c t}\right)-{ }^{2} l_{1} \sin ^{2} \theta \\
{ }^{1} l_{1} \sin { }^{1} \theta+{ }^{2} l_{1} \sin { }^{2} \theta \leq 0.75 b \\
{ }^{1} t \leq \frac{1}{5}{ }^{1} l_{1} ; \quad{ }^{1} t \leq \frac{1}{5}{ }^{1} l_{2} \\
{ }^{2} t \leq \frac{1}{5}{ }^{2} l_{1} ; \quad{ }^{2} t \leq \frac{1}{5}{ }^{2} l_{2} \\
2 l_{1} \cos { }^{1} \theta \leq \frac{a}{2^{1} n} \\
2{ }^{2} l_{1} \cos { }^{2} \theta \leq \frac{a}{2^{2} n} \\
\max \sigma_{v} \leq \sigma_{y} \\
\beta=10^{\circ}
\end{array}\right.
$$

Here, the parameters $h_{a c t}, f_{\text {safety }}$ and $L_{a c t}$ correspond to the height of actuator, the safety factor preventing interference and the actuation location, respectively. These parameters are fixed in the optimisation with values of $0.02 \mathrm{~m}, 1.5$ and 0.3 . The yield stress, $\sigma_{y}$, is assumed to be $30 \mathrm{MPa}$ in the current study. The Young's modulus of the material is $3 \mathrm{GPa}$ to simulate $\mathrm{ABS}$ plastic.

The Matlab® GA optimisation toolbox [105] is applied to find the minimum actuation force required for a specific rotation angle. The entire height of the compliant structure will be influenced by the optimal variables, and thus a series of optimisation cases are performed to find the relationship between the height of the 
compliant structure, $b$, and entire height, $B$. Before the height is determined, the width of the corrugated panels has been found irrelevant to the optimal variables since the equivalent model of the corrugated panel is inherently a 2-dimensional model. A sequence of optimisation cases with the same Matlab GA setup and state is performed to verify the assumption. While the required actuation force changes with the width of the corrugated panels, the optimised variables have almost no difference. With this assumption, the relationship between the entire height of the compliant structure $B$ and the distance between two corrugated panels $b$ is shown in Figure 6.2(a), which gives a linear trend between them. Then, the compliant structure is accommodated within the airfoil, as shown in Figure 6.2(b). The parameter $b$ can be interpolated according to the entire height $B$, and then the width of the corrugated panels can be determined according to the airfoil thickness. The width of the compliant structure is $0.0875 \mathrm{~m}$ in these optimisation cases.
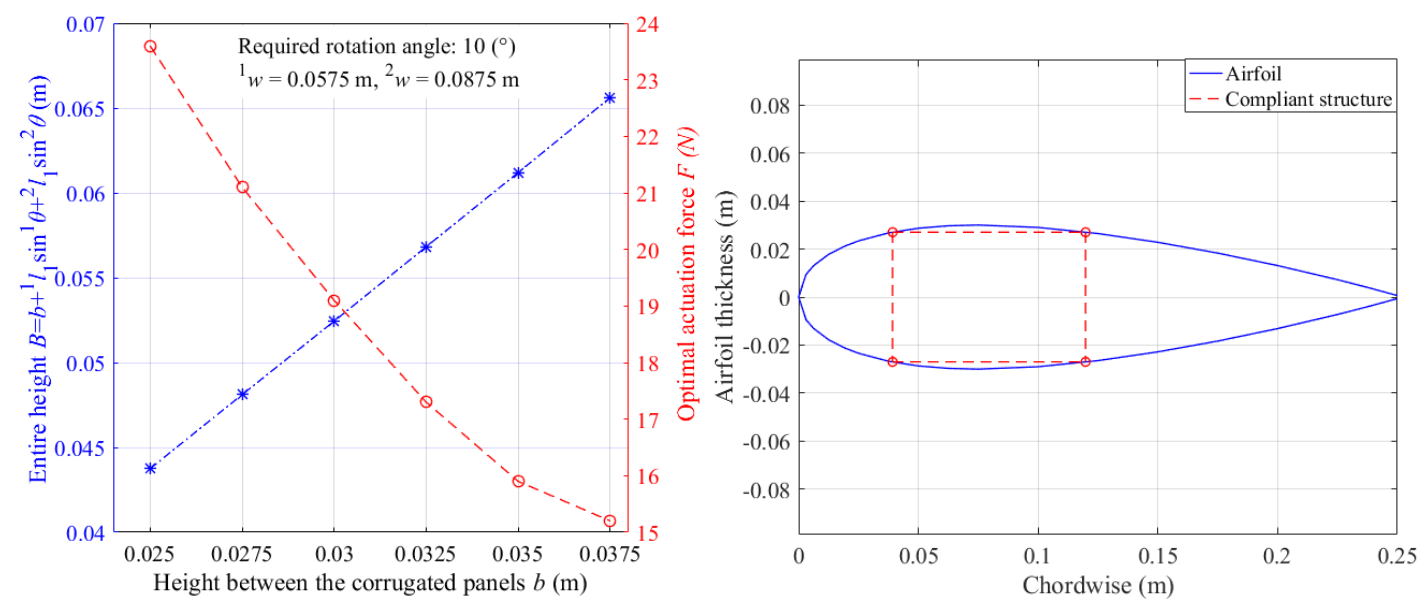

Figure 6.2 (a) Optimised height and actuation force, (b) Compliant structure in the airfoil.

In the current study, the entire height of the compliant structure is $0.0524 \mathrm{~m}$, and the width of the compliant structure accounts for around $35 \%$ of the chord, which starts at $15 \%$. Minor modifications are made considering the installation and the other details, which are not the focus of this thesis. The optimised variables of the corrugated panels are listed in Table 6.1 and the geometry is plotted in Figure 6.3(a). 

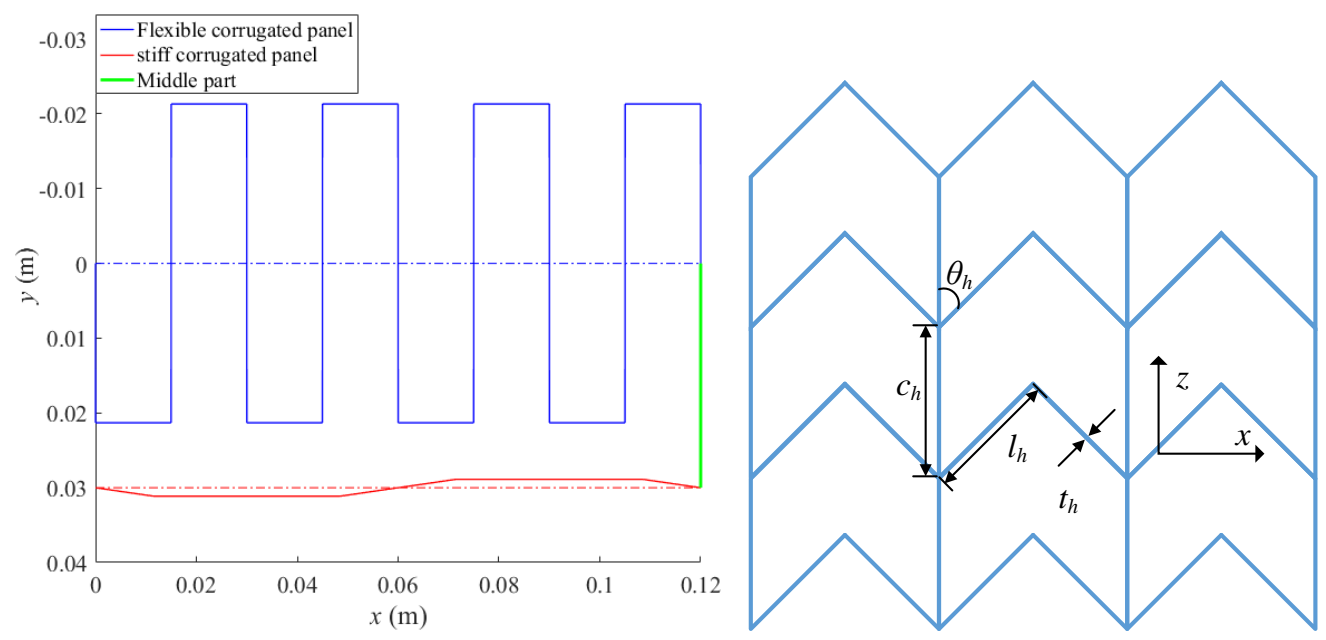

Figure 6.3 (a) Compliant structure based on corrugated panels, (b) Flexible honeycomb structure

The optimised results show that the optimisation provides a much more flexible upper corrugated panel compared to the lower panel, which introduces a significant stiffness asymmetry in the compliant structure.

\begin{tabular}{|c|c|c|c|}
\hline \multicolumn{2}{|c|}{ Upper corrugated panel } & \multicolumn{2}{c|}{ Lower corrugated panel } \\
\hline${ }^{1} l_{1}$ & $0.0213 \mathrm{~m}$ & ${ }^{2} l_{1}$ & $0.0115 \mathrm{~m}$ \\
\hline${ }^{1} \theta$ & $89.98^{\circ}$ & ${ }^{2} \theta$ & $5.60^{\circ}$ \\
\hline${ }^{1} n$ & 4 & ${ }^{2} n$ & 1 \\
\hline${ }^{1} t$ & $0.0015 \mathrm{~m}$ & ${ }^{2} t$ & $0.0015 \mathrm{~m}$ \\
\hline${ }^{1} \overline{E A}$ & $54.975 \mathrm{~N}$ & ${ }^{2} \overline{E A}$ & $65513.226 \mathrm{~N}$ \\
\hline${ }^{1} \overline{E I}$ & $0.0126 \mathrm{Nm}$ & ${ }^{2} \overline{E I}$ & $0.0737 \mathrm{Nm}$ \\
\hline
\end{tabular}

Table 6.1 Optimised variables and properties of the compliant structure in the demonstration model

The thicknesses of both panels are at their lower bounds, indicating the requirement of the smallest actuation force. Also, it can be seen the entire height of the structure will be increased by ${ }^{1} l_{1}$ since the angle ${ }^{1} \theta$ is around 90 degrees but ${ }^{2} l_{1}$ has almost no influence on the entire height due to the small ${ }^{2} \theta$. The small angle in the lower corrugated panel also makes the lower aerodynamic surface flat enough. In this 
chapter, no extra cover is added to the lower panel to simplify the manufacturing.

The equivalent tension stiffness $(\overline{E A})$ and bending stiffness $(\overline{E I})$ are calculated using Equation (4.32). The equivalent tension stiffness of the lower panel is found to be three orders of magnitude higher than that of the upper panel, which reflects an extreme stiffness asymmetry. Thus, according to Equation (3.15), a substantial rotation could be expected, helping to reduce the actuation force for a specified shape change.

\subsubsection{Leading and trailing edge solution}

The leading and trailing edges of the airfoil are provided by the flexible honeycomb structure, as shown in Figure 6.3(b). The equivalent modulus of the honeycomb in the transverse direction, $E_{x}$, has been derived in [119] as

$$
E_{x}=E_{m}\left(\frac{t_{h}}{l_{h}}\right)^{3} \frac{\sin \theta_{h}}{\frac{c_{h}}{l_{h}} \cos ^{2} \theta_{h}}
$$

A sequence of optimisations is performed to minimise the modulus. In addition to the geometry constraints, the constraint of the connection to the corrugated panels is also included: the number of honeycomb units should be an integer multiple of the number of corrugation units to ensure the honeycombs are evenly connected to the corrugated panels at both leading and trailing edges, and the loads can be transferred evenly through the structure. Since four corrugations are required in the upper part, 12 honeycomb units are selected to ensure the smooth connection.

The final selection of the parameters of the flexible honeycomb structure is listed in Table 6.2. 


\begin{tabular}{|c|c|}
\hline Variable name & Selection \\
\hline$\theta_{h}$ & $13.5^{\circ}$ \\
\hline$t_{h}$ & $0.0015 \mathrm{~m}$ \\
\hline$l_{t}$ & $0.0214 \mathrm{~m}$ \\
\hline$c_{h}$ & $0.0167 \mathrm{~m}$ \\
\hline number of honeycomb units & 12 \\
\hline
\end{tabular}

Table 6.2 Selected variables of the flexible honeycomb structures

\subsubsection{Detailed FEM model verification}

A detailed finite element model is created in Abaqus ${ }^{\circledR}$. The model includes all the details of the compliant structure including the boundary conditions and actuation point. Considering the complexity of the geometry, the geometry is built in the professional design software SOLIDWORKS® [121]. Then, the geometry model is imported to Abaqus ${ }^{\circledR}$ as shown in Figure 6.4(a). The fixed boundary condition is applied to simulate the clamping provided by the nuts and bolts. Distributed loads are applied to simulate the actuation, which avoids the extreme node displacement caused by the concentrated force. Solid quadratic tetrahedral elements (C3D10) are used in this model because the tetrahedral elements are good at meshing the complex geometry and the second-order element can avoid the volumetric locking problem of the first-order element [108]. To ensure the accuracy of the numerical simulation, a mesh size of $1.5 \mathrm{~mm}$ is adopted, resulting in the 530460 elements and 946593 nodes. The mesh tool is applied to check the quality of the meshes, which ensures there is no error in the model.

Figure 6.4(b) shows the distribution of the von Mises stress. It can be seen that the high stresses are predicted at the corners of the honeycombs and corrugated panels, which might lead to failures in the tests. The axial and vertical displacements of the compliant structure are also obtained, which can be used to estimate the rotation angle of the compliant structure, although the deformation limits are determined by the material yield stress. 


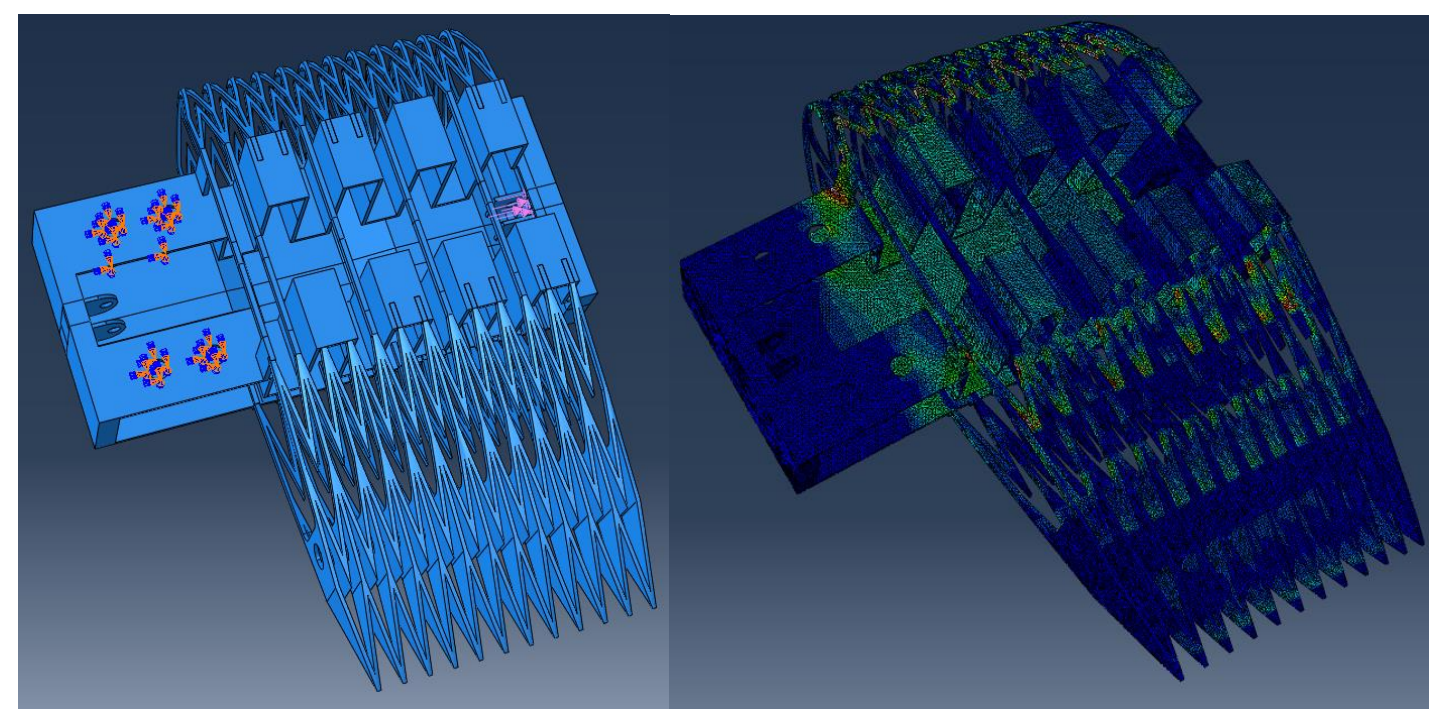

Figure 6.4 (a) Abaqus model used for numerical verification, (b) von Mises stress distribution in the deformed structure

\subsection{Model manufacture and static testing}

\subsubsection{Model manufacture}

The complex geometry obtained from the optimisation leads to problems in manufacturing, which is difficult using the conventional methods used for conventional corrugated and honeycomb structures. Due to the development of 3D printing technologies, the geometry of the functional model might be manufactured using 3D printing.

The structure was printed using an Objet printer, which prints ABS plastic and its maximum printing size is $1000 \times 1000 \times 500 \mathrm{~mm}$. The first trial runs gave a model, with significant initial deformations after printing, especially in the region of the leading and trailing edges due to the slender and thin structures. Some modifications were made to the model with more rib-like supports provided in the leading and trailing edges. As discussed in Chapter 3, the main deformation of the compliant structure is extension or compression, so the rib-like supports will not increase the actuation force since they are placed on the plane perpendicular to the axial deflections. The rib-like supports are also helpful to constrain the material during printing, which can reduce the initial deformation. For the same purpose, some very 
thin columns are added between the two upper and lower corrugation panels to prevent initial deformation, which can be removed after printing.

The current study is focused on the inner structure, rather than the skin. Hence, the skin of the demonstration model is made of silicone rubber due to availability and convenience. Considering the development of morphing skins, such as in [17], there will be better solutions if further work is continued. Although silicone rubber is very flexible, the force needed to deform the skin can still be very high due to its large area and required strain. However, very thin silicone rubber cannot be used due to the likelihood of damage during manufacturing and the requirement to carry local loads. A silicone rubber [122] with $0.5 \mathrm{~mm}$ thickness is employed after considering the maximum actuation force and other constraints.

The silicone rubber is cut to the proper size according to the model's chord and span before it is bonded to the structure using adhesive. A cyanoacrylate adhesive is applied to the surface of the structure, which works for most plastic and rubber bonding [123]. Since the silicone rubber is usually difficult to bond due to its stable characteristics, a primer is applied to the rubber surface to activate it. The structure and the silicone rubber are then assembled with a defined alignment direction. Some pressure is applied to avoid initial wrinkling. The procedure is done by hand since the adhesive is instant, and takes only a few seconds to cure. The cured adhesive then needs 24 hours to reach its maximum strength. Safety procedures provided by the adhesive's supplier were followed strictly during the whole operation and storage.

The linear actuator is installed into the morphing wingtip before the silicone rubber skin is bonded. The stroke of the actuator is $200 \mathrm{~mm}$, and the maximum actuation can be as large as $300 \mathrm{~N}$ with the gear ratio 256:1 [124]. The actuator is controlled by its own software and the extension of the actuator is given as a feedback signal.

\subsubsection{Static test}

The model for the static test is clamped to demonstrate the deflection as shown in 
Figure 6.5. The linear actuator is controlled by a control board and powered by a DC battery. A $9 \%$ initial stroke is given to the actuator to reach its installation position, at which the compliant structure is not rotated. In the static test, the position of the actuator stroke varies from $9 \%$ to $29 \%$ and then back to $9 \%$, which is recorded by a digital camera.

As shown in the figure, the stiffness asymmetry leads to a rotation angle of the compliant structure, $\beta$, as well as a change of the dihedral angle, $\gamma$, if the compliant structure works as a transition section connected to an outer fixed-geometry winglet. According to the geometry relationship, the introduced dihedral angle change $\gamma$ is larger than the rotation angle $\beta$. The angles $\beta$ and $\gamma$ are measured from the pictures, which can be as large as 20 degrees and 45 degrees respectively. While no skin is bonded to the structure during the static test, it has demonstrated the capability of the concept to change the shape of the wing.
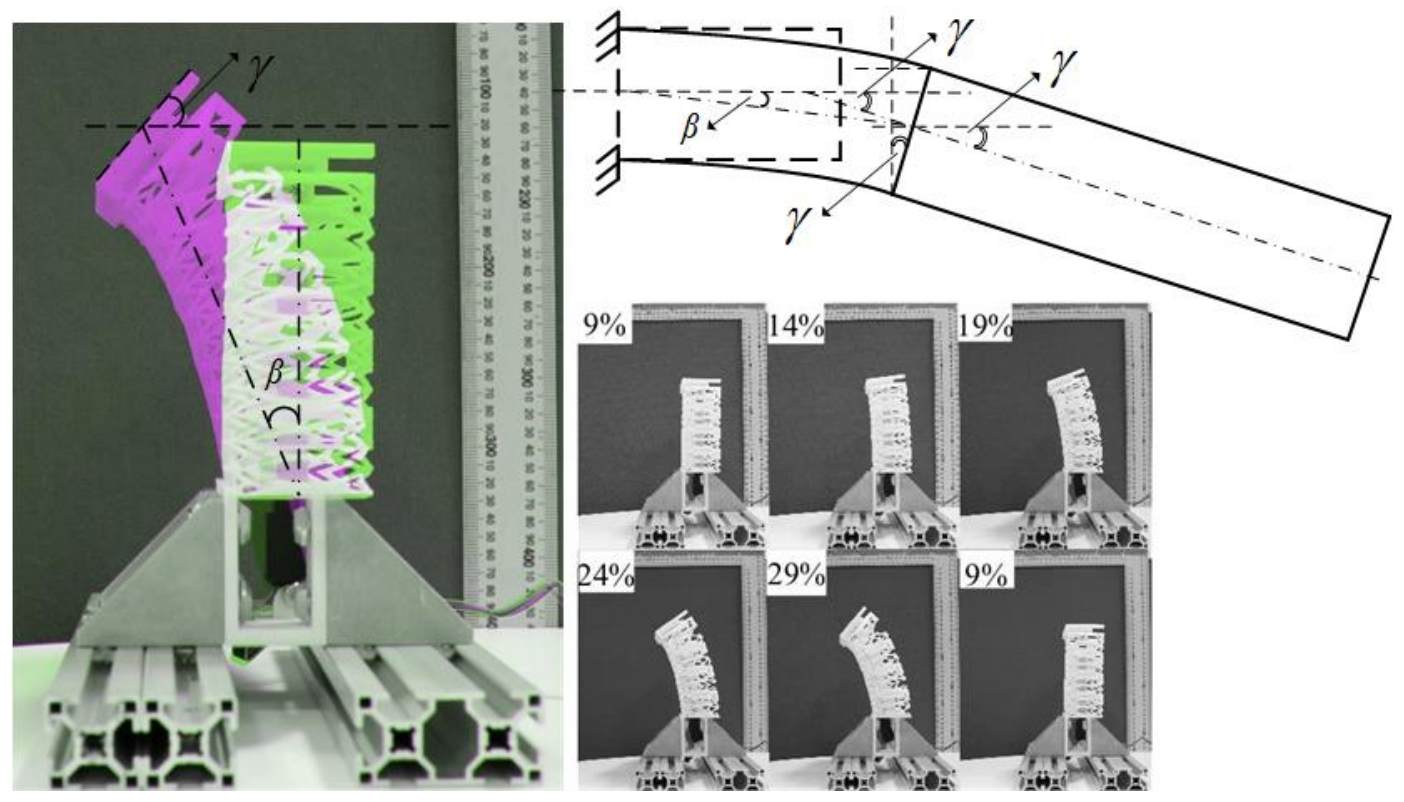

Figure 6.5 Static test demonstration when the actuator position varies from $9 \%$ to $29 \%$ of the stroke 


\subsection{Wind tunnel tests}

\subsubsection{Experimental setup and model design}

The wind tunnel tests are performed in the Swansea University low-speed wind tunnel, which is a closed return circuit wind tunnel. The maximum airspeed is $50 \mathrm{~m} / \mathrm{s}$, and the test section is $1 \mathrm{~m}$ high and $1.5 \mathrm{~m}$ wide. The analytical turbulence intensity and analytical flow uniformity is $0.175 \%$ and $0.04 \%$ respectively according to its operation manual, which is sufficient for the current study.

As shown in Figure 6.6, a balance is installed at the bottom of the test section. A rotating frame is attached to the balance, which can rotate the balance and the model together as much as $\pm 90^{\circ}$. The balance is a six-axis force plate, which can measure the three force components along the coordinate axes and three moment components around the axes. The coordinate system of the wind tunnel is shown in Figure 6.6 following the same coordinate system of the compliant structure, in which positive direction of the $x$-axis points up, the $y$-direction points to the right side if one faces the flow, and the $z$-axis corresponds to the upstream direction. The capacities of the balance are listed in Table 6.3.

\begin{tabular}{|l|c|c|c|c|c|c|}
\hline & $F_{x}(\mathrm{~N})$ & $F_{y}(\mathrm{~N})$ & $F_{z}(\mathrm{~N})$ & $M_{x}(\mathrm{Nm})$ & $M_{y}(\mathrm{Nm})$ & $M_{z}(\mathrm{Nm})$ \\
\hline Capacity & 4462 & 2231 & 2231 & 338 & 677 & 677 \\
\hline
\end{tabular}

Table 6.3 Force and moment capacity of the balance

The wind tunnel is controlled by its dedicated control software, which can read the outputs of the balance, change the airspeed and the rotation angle of the balance with the stepper motor. The wind tunnel model is installed vertically onto the balance. Thus the angle of attack of the model is changed when the rotating frame rotates.

As shown in Figure 6.6, the wind tunnel model is supported by two metal shafts. The shafts go through the bottom disc but have no contact with it, which ensures the loads on the wall is not transferred to the balance. The bottom plastic disc can rotate with the balance and the model, and the gap between the disc and the test section 
wall is sealed by an elastomer washer, which closes the test section and reduces the environmental influence. The shafts are clamped onto the balance, transferring the aerodynamic loads from the wind tunnel model to the wind tunnel balance. A gap, shorter than $0.5 \%$ of the span is provided between the bottom disc and the wind tunnel model, which makes the effect of the shafts negligible [125].
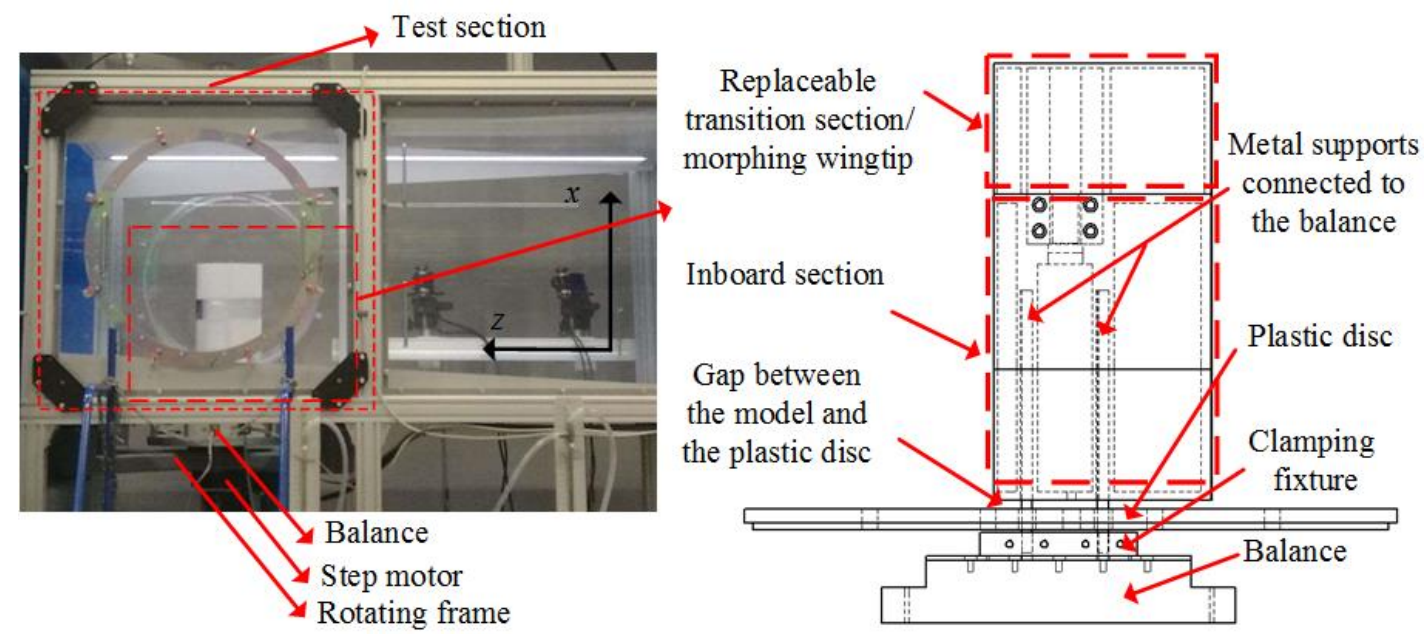

Figure 6.6 Wind tunnel test setup and the model installation

The wind tunnel model used in the tests is shown in Figure 6.7. The wind tunnel model consists of the following components: the inboard section, the transition section, the morphing wingtip and the extended outboard section. All the components are manufactured by $3 \mathrm{D}$ printing except that the extended outboard section is made of foam to reduce the weight and cost. The inboard section, transition section, and the morphing wingtip are assembled by nuts and bolts, which makes them replaceable.

The fixed-geometry inboard region helps to provide a steady inboard flow. And the transition section between the inboard section and the morphing wingtip can provide an initial dihedral angle to the wingtip. The morphing wingtip can also be installed directly onto the inboard section. In this case, the wind tunnel model has no initial dihedral angle, and the test results can be the baseline results for the validation when the actuator does not cause any deformation. The extended outboard section is connected to the morphing wingtip using carbon fibre tubes. With the outboard section, the morphing wingtip works as a transition part to change the dihedral angle 
of a fixed winglet.

The spanwise lengths of the inboard section, the morphing wingtip and the outboard section are $0.35 \mathrm{~m}, 0.15 \mathrm{~m}$ and $0.15 \mathrm{~m}$ respectively. No sweep angle or taper ratio is introduced to simplify the experiments. The NACA 0024 airfoil is used except for the small modification of the airfoil of the morphing wingtip as explained in Section 6.2. Aluminium foil tape is used to seal the gaps between the different sections and provide a smooth surface to the foam.

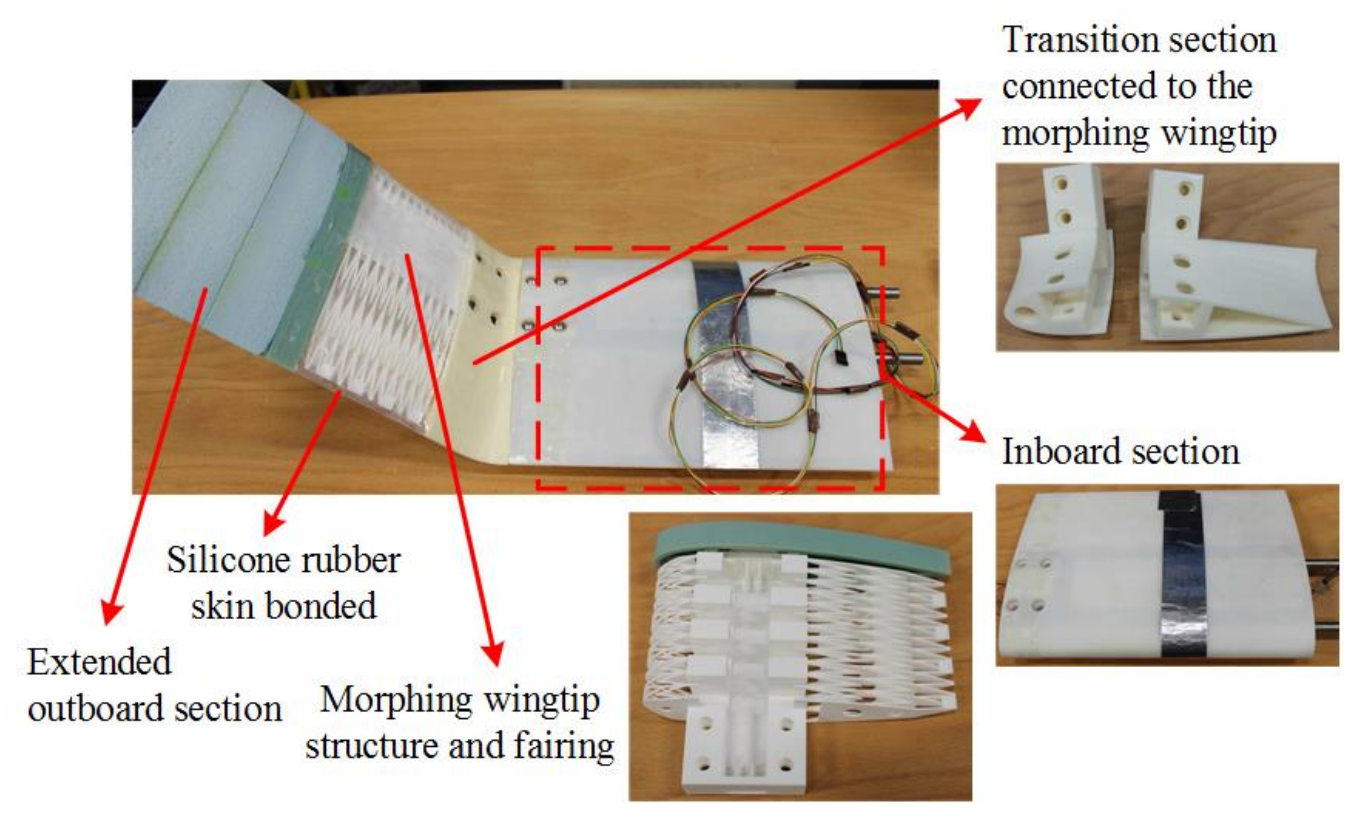

Figure 6.7 Components and assembly of the wind tunnel model

The transition section is $0.088 \mathrm{~m}$ in length. A parametric study using numerical calculation is performed to show the aerodynamic influence of different dihedral angles. A vortex lattice method based software, Tornado VLM (TVLM) [126], is applied. The TVLM software is written in Matlab®, which is sufficient and convenient for the low-speed cases. 280 panels are used to model the wing, providing enough accuracy. As shown in Figure 6.8, the lift coefficient $C_{L}$ and the rolling moment coefficient $C_{M}$ are calculated with different wingtip dihedral angles. The larget slope of $C_{L}$ and $C_{M}$ can be found when the wingtip dihedral angle is $50^{\circ}$. For the same shape change caused by the morphing wingtip, the change in aerodynamic performance can be maximised by choosing the initial dihedral angle. The current transition section leads to a $52^{\circ}$ dihedral, which is optimal considering 
the high capacity of the wind tunnel balance and emphasising the load change caused by the morphing wingtip. Different initial dihedral angles can be introduced with different transition sections if necessary. Employing the transition section also makes the assembling of the morphing wingtip easier since the silicone rubber skin can be bonded to the transition section before the morphing wingtip is installed onto the inboard section.
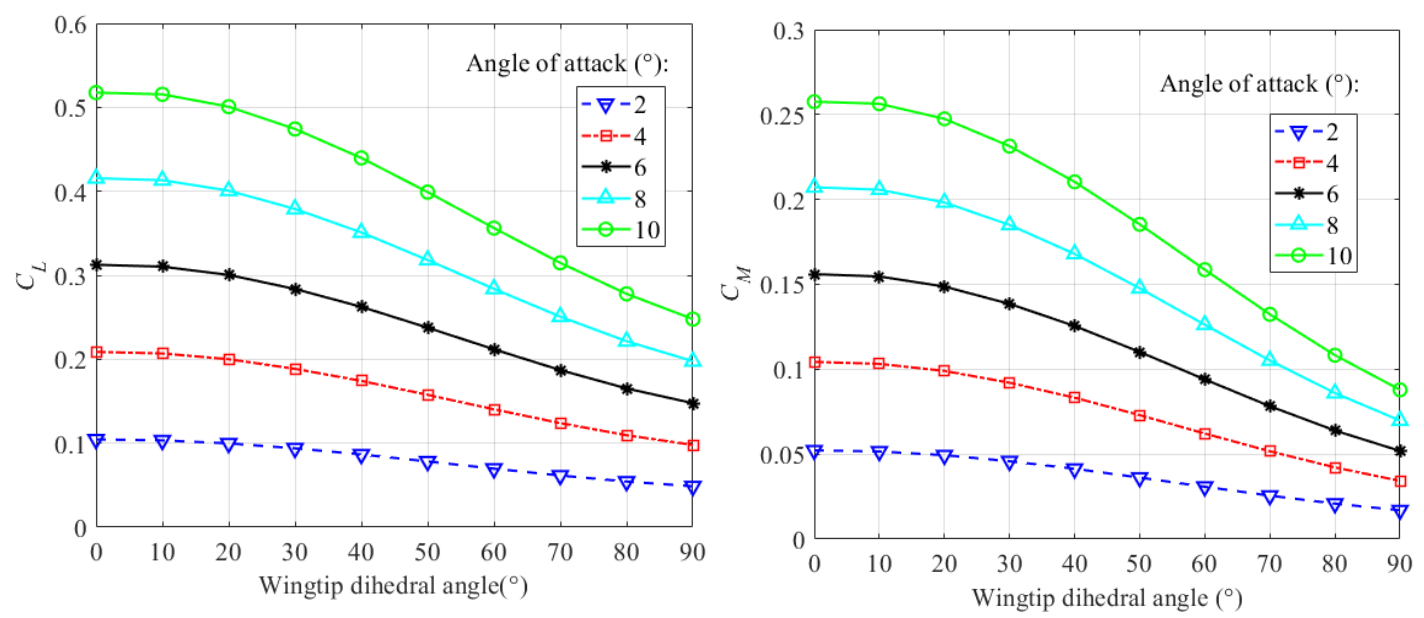

Figure 6.8 Influence of the initial dihedral angle on the (a) Lift coefficient and (b)

Rolling moment coefficient

\subsubsection{Test results and discussion}

The wind tunnel model without the transition section is tested first. The morphing wingtip is installed directly onto the inboard section. It should be noted that the morphing wingtip will lead to a curved spanwise change when the actuator extends or compresses the structure, which makes it difficult to validate the test results. Thus, the actuator does not deform the morphing wingtip for the baseline test, which leads to a wind tunnel model with zero dihedral angle.

The reference span is $0.65 \mathrm{~m}$, the reference area is $0.1625 \mathrm{~m}^{2}$ and the test speed is $20 \mathrm{~m} / \mathrm{s}$. The angle of attack varies from 0 to $10^{\circ}$ with a $2^{\circ}$ increment.

The control software of the wind tunnel takes the average value of the balance outputs and returns the differential pressure in the test section and the table rotation angle as feedback of the wind tunnel status. Corrections to the measured data are 
performed considering solid and wake blockage corrections [125].

The total blockage $\varepsilon_{T}$, is the given as

$$
\varepsilon_{T}=\varepsilon_{s b}+\varepsilon_{w b}=\frac{K_{1} \tau_{1}(\text { wing volume })}{C_{w}^{3 / 2}}+\frac{S_{w}}{4 C_{w}} C_{D u}
$$

Here, the solid and wake blockage is denoted as $\varepsilon_{s b}$ and $\varepsilon_{w b}$ respectively. The body shape factor $K_{1}$ is 1.10 , the factor $\tau_{1}$, which depends on the test section shape and the model span to tunnel width ratio, is 0.925 . The wind tunnel test section (cross-section) area $C_{w}$ is $1.5 \mathrm{~m}^{2}$, and the wing area is denoted as $S_{w}$. The subscript ' $u$ ' is used to indicate the measured data.

The blockage is found to be less than $0.7 \%$. The correction of the velocity, $V$, and the dynamic pressure, $q$, is then made as

$$
\begin{aligned}
& V=V_{u}\left(1+\varepsilon_{T}\right) \\
& q=q_{u}\left(1+\varepsilon_{T}\right)^{2}
\end{aligned}
$$

The test results are compared to the results from the numerical calculation in TVLM.
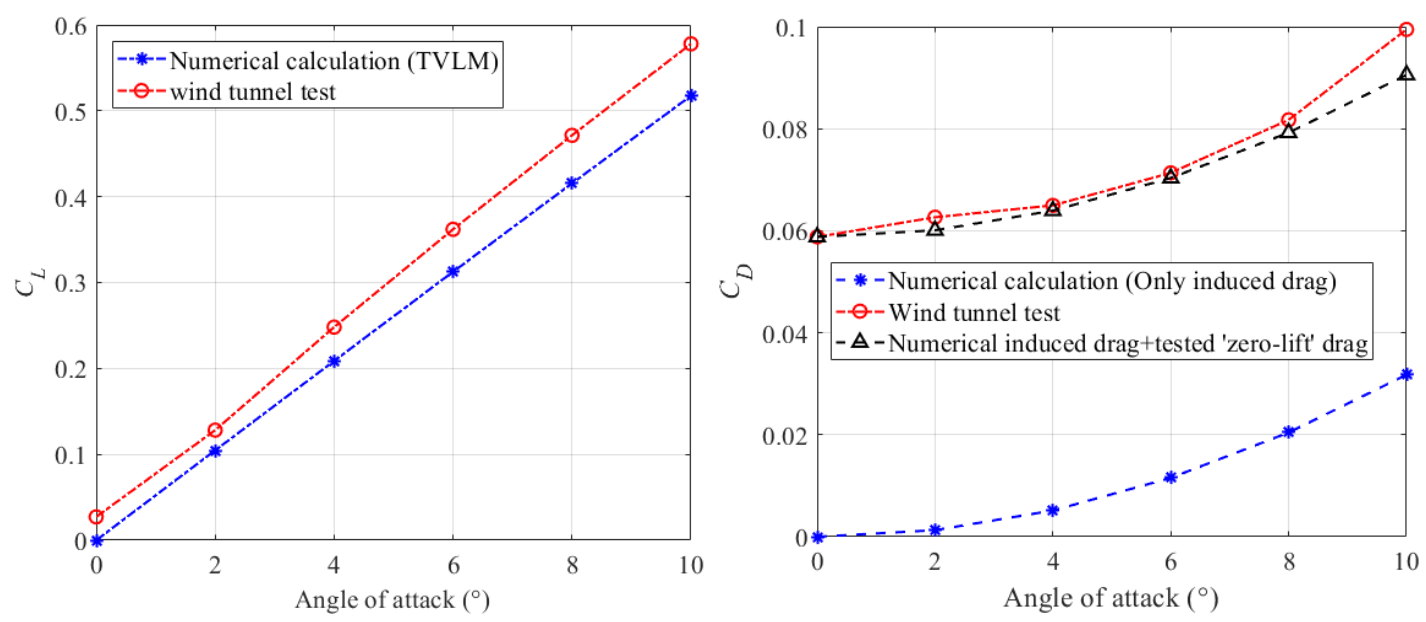

Figure 6.9 Comparisons of the wind tunnel test results: (a) Lift coefficient, (b) Drag coefficient

Figure 6.9 shows that the baseline model generates a small lift when the angle of attack is 0 , although the airfoil NACA 0024 is symmetrical. The lift could be due to the modification of the airfoil by the morphing wingtip, which changes the 
symmetry of the airfoil. Apart from this offset, the lift coefficient has a close trend compared to the numerical calculation. TVLM only calculates the induced drag, while the wind tunnel test will measure all the drag components. The 'zero-lift' drag is estimated roughly using the measured drag coefficient when the angle of attack is 0 , although there exists a small lift. Adding the 'zero-lift' drag to the numerically calculated drag will provide a drag that is close to the wind tunnel test data, which at least shows a similar trend of the drag change. Generally, the wind tunnel tests of the baseline model provide reasonable results compared to the numerical calculation.

The test of the morphing wingtip is then performed with the transition section installed. In this test, the morphing wingtip is made of Polyamide using the selective laser sintering method. Polyamide has a higher tensile strength than ABS plastic but has a lower modulus than ABS, which could lead to a larger change in the shape of the morphing structure. The silicone rubber is bonded after the actuator is installed. As shown in Figure 6.10, the actuator extension causes rotation of the morphing wingtip, which increases the dihedral angle of the outboard section. Estimation from the picture shows a $20^{\circ}$ change of the dihedral angle can be achieved. Although an even larger angle change can be obtained, the actuator extension is limited to $12 \%$ of the stroke to ensure the morphing wingtip does not fail in the test.
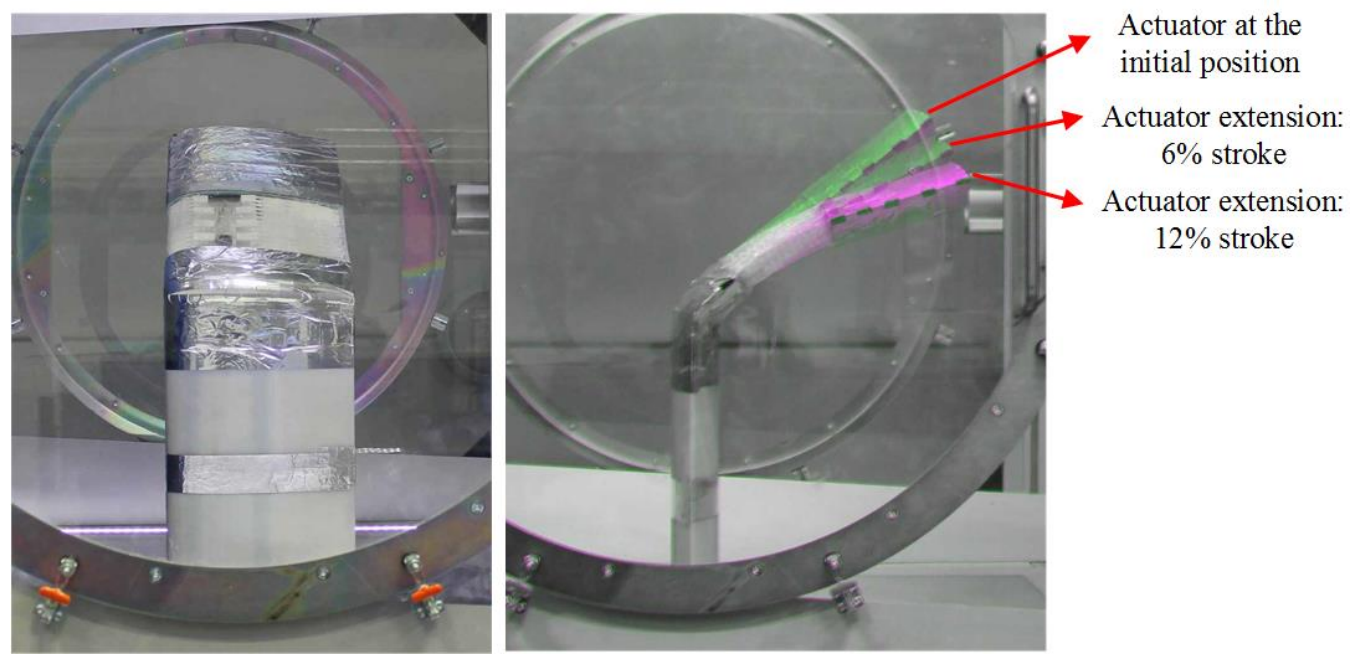

Figure 6.10 The wind tunnel model: (a) Front view, and (b) Side view with different the actuator extensions 
The test is performed with an airspeed $20 \mathrm{~m} / \mathrm{s}$. The angle of attack of the wind tunnel models varies from 0 to $10^{\circ}$. The reference span is $0.734 \mathrm{~m}$ and the reference area is $0.1835 \mathrm{~m}^{2}$. Corrections of the data are also applied using the same method to the baseline test, and the blockage is still quite small. The change of aerodynamic performance caused by the morphing wingtip is shown in Figure 6.11. The rolling moment measured by the balance is based on the reference plane located at the centre of the balance, and hence the rolling moment is actually overestimated. The reduction of the lift coefficient $C_{L}$ and the rolling moment coefficient $C_{M}$ is demonstrated in the test. For example, when the angle of attack is $6^{\circ}$, a $12 \%$ reduction of the $C_{L}$ and a $15 \%$ reduction of $C_{M}$ can be obtained if the actuator extension is $12 \%$ of the stroke. Comparing the results caused by different actuation extensions shows the reduction of the aerodynamic loads can be increased when the actuator causes a larger shape change.
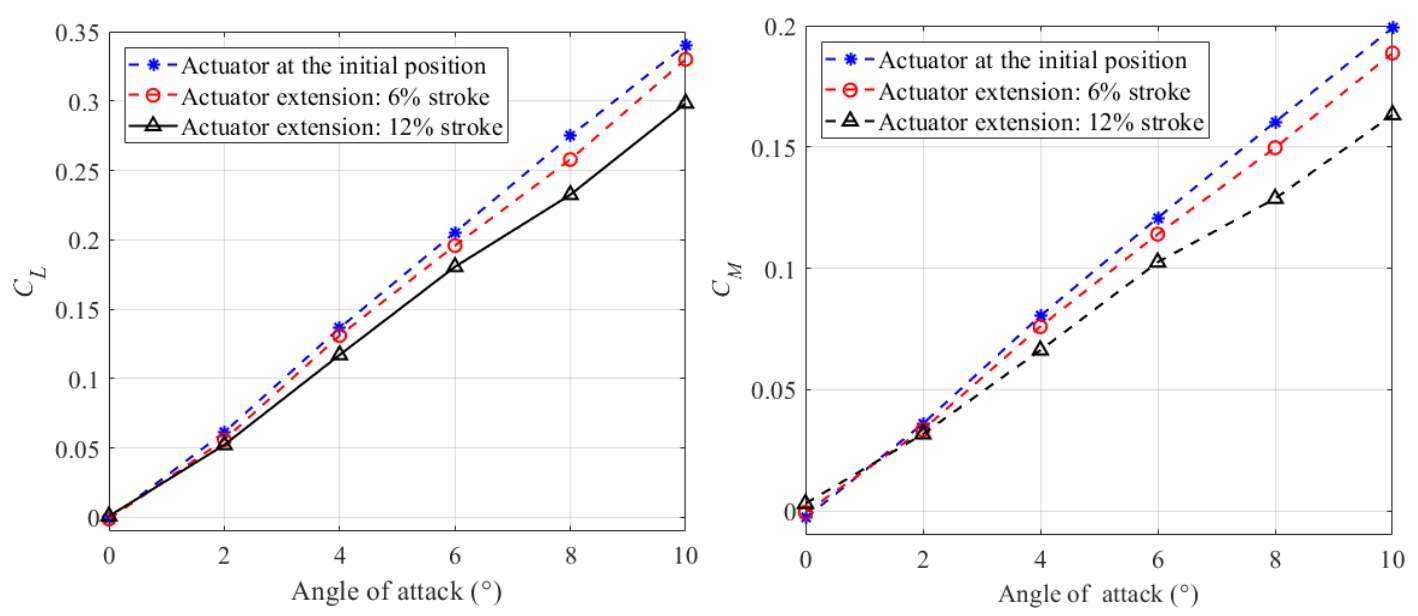

Figure 6.11 Change of (a) lift coefficient and (b) rolling moment coefficient caused by the morphing wingtip

The reduction of the rolling moment also indicates the reduction of the wing root bending moment, which is one of the performance critera in Section 2.2. Although the wind tunnel model has a different geometry compared to the model used in Chapter 2, the decrease of the rolling moment shows the potential to reduce the wing root bending moment with the morphing wingtip. Further tests will be required to validate the aerodynamic study in Chapter 2 comprehensively, considering the 
limitation of the current test speed and the half-wing wind tunnel model, which is beyond the scope of the thesis.

\subsection{Summary}

In this chapter, the development of a demonstration model of the morphing wingtip is introduced. The demonstration model consists of the primary component, which is the compliant structure based on unsymmetrical stiffness, and the secondary components, which use flexible honeycomb structures to provide the airfoil shape.

Trapezoidal corrugated panels are used in the compliant structures. The equivalent model using the stiffness matrix of the corrugated panels is applied in the optimisation to find the optimal stiffness allocation in the structure. To fit within the thickness of the airfoil, a sequence of optimisation cases is performed to find the optimum height of the compliant structure. Flexible honeycomb structures are used in the leading and trailing edges, which are evenly connected to the corrugated panels after the optimisation of the honeycomb structures is performed. The optimisation gives an extreme stiffness asymmetry, which has three orders of magnitude difference between the stiffness of the upper and lower panels and explains the minimal actuation force. The optimisation results also highlight the influence of the unsymmetrical stiffness, and validates the proposed concept.

The functional model is manufactured using 3D printing, and the static tests validate the deformation of the model, which is driven by a linear actuator. The rotation angle is recorded while a larger dihedral angle is found if the proposed model works as a transition section connected to an outboard fixed-geometry winglet, which shows a promising structural solution for a morphing winglet.

The wind tunnel test of the demonstration model validates the potential of the morphing winglet in a low-speed flight condition. A baseline test is first performed with zero dihedral angle. Numerical calculation is applied to compare the test results. The test of the morphing wingtip model shows that the aerodynamic performance is 
affected as the actuator works changes the dihedral angle.

Although the demonstration model is only tested at a low-speed and requires more improvement for a practical industry application, the current progress and results have validated the morphing wingtip concept based on unsymmetrical stiffness. Since the reduction of the aerodynamic loads is measured when the morphing wingtip increases the dihedral angle, potential applications of the design could be on the ground to reduce the span, as well as for load alleviation. 


\section{Chapter 7 Conclusions}

\subsection{Contributions}

The thesis presents the process of developing a morphing aircraft concept. The morphing concept is based on morphing wingtip devices (morphing winglet), which aims for a significant aerodynamic performance improvement to the aircraft while keeping the side effects (weight increase, system complexity) as small as possible. Aerodynamic analysis has been performed first, which reflects the significance of changing the dihedral angle of morphing winglets. Compliant structures are applied to provide the continuous shape change rather than the discrete deformation.

The main contributions of the thesis can be categorised into three aspects:

1) A compliant structure concept based on unsymmetrical stiffness

A novel compliant structure is proposed to achieve the rotation of the structure. A simplified model of the compliant structure has been built in the $2 \mathrm{D}$ situation. Analytical expressions of the compliant structure have been derived, in which the total stiffness, $K_{t}$, is helpful to reduce the deflections under the aerodynamic load. However, the ratio between the extension stiffnesses of the different parts, $r_{s}$, can induce a rotation angle of the compliant structure when the structure is actuated.

A specific stiffness allocation could be found to achieve the two goals simultaneously, which demands the optimisation of the detailed structure. The optimisation performed in Chapters 5 and 6 further investigates the compliant structure, which minimises the actuation force when meeting the requirements of carrying loads and changing shape.

2) An equivalent model of the corrugated panels developed for the compliant structure 
The equivalent model of the corrugated panel is created by obtaining its deflections under different load cases. The corrugated panel can be seen as an equivalent beam, for which the stiffness matrix is built. The equivalent beam model has the same degrees of freedom to an Euler beam element but has coupling components between the vertical deflections and the axial forces. By assembling the stiffness matrix of the corrugated panels and the other parts of the structure, the equivalent model of the entire compliant structure is obtained. The equivalent model has been verified by numerical simulation using detailed finite element models in commercial software and tensile experiments of corrugated panels.

3) The study of the morphing wingtip devices based on the proposed compliant structure

A system level study is conducted to integrate the compliant structure concept into a baseline design in Chapter 3. The weight increase due to the retrofitted morphing winglet is estimated. After optimising the compliant structure using the equivalent models in Chapter 5, the weight increase caused by the actuation system can be reduced significantly, which improves the system level benefits.

A demonstration model is designed, analysed, manufactured and tested in Chapter 6. Static tests and wind tunnel tests have been conducted to validate the model. A rotation angle of $20^{\circ}$ is measured in the static test. In the wind tunnel tests, the change of aerodynamic performance caused by the morphing wingtip has been demonstrated.

\subsection{Future work}

Although much work has been done on the topic, there still exist many opportunities to improve the current work and further investigate the potential of the morphing winglet in several aspects.

1) Introducing the twist deformation by using two compliant structures

The idea to introduce a twist deformation to the winglet is very briefly mentioned in 
the numerical simulation of a large wing structure in Section 3.4. Two compliant structures could be applied to introduce two different rotation angles, which eventually introduce a twist angle of the winglet. However, the idea has not been fully investigated and clarified. By enabling both dihedral and twist angle to be changed, an improved aerodynamic performance could be achieved.

\section{2) Reducing actuation force}

The actuation force has been minimised in this thesis. However, further improvement could be made by adopting some additional mechanisms, such as using passive energy balancing. It might be challenging to apply the mechanism in the limited space of the wing/wingtip, and further research needs to be done considering this constraint. Another approach is to make use of the novel actuators. Such actuators should be selected based on system level benefits considering energy requirements, system complexity, and reliability.

\section{3) Load alleviation}

Passive load alleviation using wingtip devices has been reviewed in the thesis. Load alleviation approaches could be tested using active controlled wingtips, which would change the dihedral and twist angle. The topic would be challenging concerning the response time of the active control system, and the required control algorithm.

4) Multi-disciplinary optimisation of the morphing winglet with more practical data

The thesis intends to take as many aspects of the morphing concept as possible into account. But the optimisation of the compliant structure is still a structural optimisation, which could be further modified to a multi-disciplinary optimisation. By linking the aerodynamic module to the optimisation scheme, further improvement of the morphing winglet performance could be expected.

The data applied in the thesis is acquired from open sources although it represents typical cases. It would be meaningful to apply the optimisation with more practical data, such as using the real aircraft geometry and flight condition parameters. 


\section{References}

1. Weisshaar, T.A., Morphing aircraft technology-new shapes for aircraft design. 2006, ADA479821.

2. Jha, A.K. and Kudva, J.N. Morphing aircraft concepts, classifications, and challenges. Proceedings of Smart Structures and Materials 2004: Industrial and Commercial Applications of Smart Structures Technologies, Vol. 5388, p. 213-225, July 29, 2004. SPIE.

3. Barbarino, S., Bilgen, O., Ajaj, R.M., Friswell, M.I., and Inman, D.J., A review of morphing aircraft. Journal of Intelligent Material Systems and Structures, 2011. 22(9): p. 823-877.

4. Clarivate Analytics. Web of Science 2017, from:

https://apps.webofknowledge.com/.

5. Crouch, T.D. Ader Avion III. from:

https://www.britannica.com/topic/Ader-Avion-III.

6. NASA. Wing warping. from:

https://www.grc.nasa.gov/WWW/Wright/airplane/warp.html.

7. Savine, A. Russian Aviation Museum. from:

http://ram-home.com/ram-old/rk.html.

8. Norman, D., Hynes, R., and Gangsaas, D. An integrated maneuver enhancement and gust alleviation mode for the AFTI/F-111 MAW aircraft. Guidance and Control Conference. Gatlinburg, TN, United States, 1983, AIAA.

9. Gilbert, W.W., Mission Adaptive Wing System for Tactical Aircraft. Journal of Aircraft, 1981. 18(7): p. 597-602.

10. Decamp, R. and Hardy, R. Mission adaptive wing advanced research concepts. 11th Atmospheric Flight Mechanics Conference. Seattle, WA, United States, AIAA.

11. Kudva, J.N., Martin, C.A., Scherer, L.B., Jardine, A.P., McGowan, A.-M.R., 
Lake, R.C., Sendeckyj, G.P., and Sanders, B.P. Overview of the DARPA/AFRL/NASA Smart Wing program. Proceedings of 1999 Symposium on Smart Structures and Materials, Vol. 3674, p. 230-237, Newport Beach, CA, United States. SPIE.

12. Kudva, J.N., Overview of the DARPA smart wing project. Journal of Intelligent Material Systems and Structures, 2004. 15(4): p. 261-267.

13. Flanagan, J., Strutzenberg, R., Myers, R., and Rodrian, J. Development and Flight Testing of a Morphing Aircraft, the NextGen MFX-1. 48th AIAA/ASME/ASCE/AHS/ASC Structures, Structural Dynamics, and Materials Conference. Honolulu, Hawaii, United States, April 23-26, 2007, AIAA.

14. Bowman, J., Sanders, B., Cannon, B., Kudva, J., Joshi, S., and Weisshaar, T. Development of Next Generation Morphing Aircraft Structures. 48th AIAA/ASME/ASCE/AHS/ASC Structures, Structural Dynamics, and Materials Conference. Honolulu, Hawaii, United States, April 23-26, 2007, AIAA.

15. Andersen, G., Cowan, D., and Piatak, D. Aeroelastic Modeling, Analysis and Testing of a Morphing Wing Structure. 48th AIAA/ASME/ASCE/AHS/ASC Structures, Structural Dynamics, and Materials Conference. Honolulu, Hawaii, United States, April 23-26, 2007, AIAA.

16. Westfall, J., Canfield, R., Joo, J., and Sanders, B. Multi-Disciplinary Optimization of a Distributed Actuation System in a Flexible Morphing Wing. 48th AIAA/ASME/ASCE/AHS/ASC Structures, Structural Dynamics, and Materials Conference. Honolulu, Hawaii, United States, April 23-26, 2007, AIAA.

17. Thill, C., Etches, J., Bond, I., Potter, K., and Weaver, P., Morphing skins. The Aeronautical Journal, 2008. 112(1129): p. 117-139.

18. Woods, B.K.S., Bilgen, O., and Friswell, M.I., Wind tunnel testing of the fish bone active camber morphing concept. Journal of Intelligent Material Systems and Structures, 2014. 25(7): p. 772-785.

19. Woods, B.K.S. and Friswell, M.I. Preliminary investigation of a fishbone active camber concept. Proceedings of ASME 2012 Conference on Smart Materials, Adaptive Structures and Intelligent Systems, Vol. p. 555-563, Stone Mountain, Georgia, United States, September 19-21, 2012. ASME. 
20. Woods, B.K.S., Dayyani, I., and Friswell, M.I., Fluid/structure-interaction analysis of the fish-bone-active-camber morphing concept. Journal of Aircraft, 2014. 52(1): p. 307-319.

21. Chen, S., Chen, Y., Zhang, Z., Liu, Y., and Leng, J., Experiment and analysis of morphing skin embedded with shape memory polymer composite tube. Journal of Intelligent Material Systems and Structures, 2014. 25(16): p. 2052-2059.

22. Shan, Y., Philen, M.P., Bakis, C.E., Wang, K.-W., and Rahn, C.D., Nonlinear-elastic finite axisymmetric deformation of flexible matrix composite membranes under internal pressure and axial force. Composites Science and Technology, 2006. 66(15): p. 3053-3063.

23. Campanile, L.F., Initial Thoughts on Weight Penalty Effects in Shape-adaptable Systems. Journal of Intelligent Material Systems and Structures, 2005. 16(1): p. 47-56.

24. Skillen, M.D. and Crossley, W.A., Modeling and optimization for morphing wing concept generation. 2007, NASA/CR-2007-214860.

25. Skillen, M.D. and Crossley, W.A., Modeling and Optimization for Morphing Wing Concept Generation II. Part 1; Morphing Wing Modeling and Structural Sizing Techniques. 2008, NASA/CR-2008-214902.

26. Skillen, M.D. and Crossley, W.A., Morphing Wing Weight Predictors and Their Application in a Template-Based Morphing Aircraft Sizing Environment II. Part 2; Morphing Aircraft Sizing via Multi-level Optimization. 2008, NASA/CR-2008-214903.

27. Crossley, W.A., Skillen, M.D., Frommer, J.B., and Roth, B.D., Morphing aircraft sizing using design optimization. Journal of Aircraft, 2011. 48(2): p. $612-622$.

28. Buannic, N., Cartraud, P., and Quesnel, T., Homogenization of corrugated core sandwich panels. Composite Structures, 2003. 59(3): p. 299-312.

29. Chang, W.-S., Ventsel, E., Krauthammer, T., and John, J., Bending behavior of corrugated-core sandwich plates. Composite Structures, 2005. 70(1): p. 81-89.

30. Biancolini, M., Evaluation of equivalent stiffness properties of corrugated board. 
Composite structures, 2005. 69(3): p. 322-328.

31. Yokozeki, T., Takeda, S.-i., Ogasawara, T., and Ishikawa, T., Mechanical properties of corrugated composites for candidate materials of flexible wing structures. Composites Part A: applied science and manufacturing, 2006. 37(10): p. $1578-1586$.

32. Thill, C., Etches, J.A., Bond, I.P., Potter, K.D., and Weaver, P.M., Composite corrugated structures for morphing wing skin applications. Smart Materials and Structures, 2010. 19(12): p. 124009-124018.

33. Dayyani, I., Haddad Khodaparast, H., Woods, B.K.S., and Friswell, M.I., The design of a coated composite corrugated skin for the camber morphing airfoil. Journal of Intelligent Material Systems and Structures, 2014. 26(13): p. 1592-1608.

34. Previtali, F., Molinari, G., Arrieta, A.F., Guillaume, M., and Ermanni, P., Design and experimental characterisation of a morphing wing with enhanced corrugated skin. Journal of Intelligent Material Systems and Structures, 2015. 27(2): p. 278-292. 35. Alessandro, A., Stephane, F., Elena, B., Paolo, B., and Giuseppe, S., Design and manufacturing of skins based on composite corrugated laminates for morphing aerodynamic surfaces. Smart Materials and Structures, 2017. 26(4): p. 045024.

36. Dayyani, I., Shaw, A.D., Saavedra Flores, E.I., and Friswell, M.I., The mechanics of composite corrugated structures: A review with applications in morphing aircraft. Composite Structures, 2015. 133: p. 358-380.

37. Gibson, L.J. and Ashby, M.F., Cellular Solids: Structure and Properties. Second ed. Cambridge Solid State Science Series. 1997, Cambridge: Cambridge University Press.

38. Bubert, E.A., Woods, B.K., Lee, K., Kothera, C.S., and Wereley, N.M., Design and fabrication of a passive $1 D$ morphing aircraft skin. Journal of Intelligent Material Systems and Structures, 2010. 21(17): p. 1699-1717.

39. Olympio, K.R. and Gandhi, F., Flexible Skins for Morphing Aircraft Using Cellular Honeycomb Cores. Journal of Intelligent Material Systems and Structures, 2010. 21(17): p. 1719-1735.

40. Olympio, K.R., Gandhi, F., Asheghian, L., and Kudva, J., Design of a Flexible 
Skin for a Shear Morphing Wing. Journal of Intelligent Material Systems and Structures, 2010. 21(17): p. 1755-1770.

41. Wang, C., Qiu, J., Nie, R., Ji, H., and Deng, W., Development of a Morphing Skin Based on the Honeycomb Reinforced Elastomer. Computers, Materials, \& Continua, 2012. 32(1): p. 61-79.

42. Bilgen, O., Kochersberger, K., Diggs, E., Kurdila, A., and Inman, D. Morphing Wing Micro-Air-Vehicles via Macro-Fiber-Composite Actuators. 48th AIAA/ASME/ASCE/AHS/ASC Structures, Structural Dynamics, and Materials Conference. Honolulu, Hawaii, United States, April 23-26, 2007, AIAA.

43. Bilgen, O., Kochersberger, K.B., and Inman, D.J., Macro-fiber composite actuators for a swept wing unmanned aircraft. The Aeronautical Journal, 2009. 113(1144): p. 385-395.

44. Bilgen, O. and Friswell, M.I., Implementation of $a$ Continuous-Inextensible-Surface Piezocomposite Airfoil. Journal of Aircraft, 2012. 50(2): p. 508-518.

45. Mabe, J., Calkins, F., and Butler, G. Boeing's Variable Geometry Chevron, Morphing Aerostructure for Jet Noise Reduction. 47th AIAA/ASME/ASCE/AHS/ASC Structures, Structural Dynamics, and Materials Conference. Newport, Rhode Island, United States, May 1-4, 2006, AIAA.

46. Bartley-Cho, J.D., Wang, D.P., Martin, C.A., Kudva, J.N., and West, M.N., Development of High-rate, Adaptive Trailing Edge Control Surface for the Smart Wing Phase 2 Wind Tunnel Model. Journal of Intelligent Material Systems and Structures, 2004. 15(4): p. 279-291.

47. Lu, X., Hu, J., Yang, L., and Zhao, C., A novel in-plane mode rotary ultrasonic motor. Chinese Journal of Aeronautics, 2014. 27(2): p. 420-424.

48. Philip, J., F.W. Lanchester and the Great Divide Journal of Aeronautical History 2014: p. 55-105 No. 2014/02.

49. Chambers, J.R., Concept to Reality: Contributions of the Langley Research Center to US Civil Aircraft of the 1990s. NASA history series, 2003, NASA/SP-2003-4529. 
50. Whitcomb, R.T., A design approach and selected wind tunnel results at high subsonic speeds for wing-tip mounted winglets. 1976, NASA-TN-D-8260, L-10908.

51. Pfeiffer, N. Numerical Winglet Optimization. 42nd AIAA Aerospace Sciences Meeting and Exhibit. Reno, Nevada, United States, January 5-8, 2004, AIAA.

52. Takenaka, K., Hatanaka, K., Yamazaki, W., and Nakahashi, K., Multidisciplinary Design Exploration for a Winglet. Journal of Aircraft, 2008. 45(5): p. 1601-1611.

53. Panagiotou, P., Kaparos, P., and Yakinthos, K., Winglet design and optimization for a MALE UAV using CFD. Aerospace Science and Technology, 2014. 39(Supplement C): p. 190-205.

54. Maughmer, M.D., Swan, T.S., and Willits, S.M., Design and Testing of a Winglet Airfoil for Low-Speed Aircraft. Journal of Aircraft, 2002. 39(4): p. 654-661.

55. Halpert, J., Prescott, D., Yechout, T., and Arndt, M. Aerodynamic Optimization and Evaluation of KC-135R Winglets, Raked Wingtips, and a Wingspan Extension. 48th AIAA Aerospace Sciences Meeting Including the New Horizons Forum and Aerospace Exposition. Orlando, Florida, United States, January 4-7, 2010, AIAA.

56. Büscher, A., Radespiel, R., and Streit, T., Modelling and design of wing tip devices at various flight conditions using a databased aerodynamic prediction tool. Aerospace Science and Technology, 2006. 10(8): p. 668-678.

57. Koreanschi, A., Sugar Gabor, O., Acotto, J., Brianchon, G., Portier, G., Botez, R.M., Mamou, M., and Mebarki, Y., Optimization and design of an aircraft's morphing wing-tip demonstrator for drag reduction at low speed, Part I Aerodynamic optimization using genetic, bee colony and gradient descent algorithms. Chinese Journal of Aeronautics, 2017. 30(1): p. 149-163.

58. Koreanschi, A., Gabor, O.S., Acotto, J., Brianchon, G., Portier, G., Botez, R.M., Mamou, M., and Mebarki, Y., Optimization and design of an aircraft's morphing wing-tip demonstrator for drag reduction at low speeds, Part II - Experimental validation using Infra-Red transition measurement from Wind Tunnel tests. Chinese Journal of Aeronautics, 2017. 30(1): p. 164-174.

59. Vasista, S., Riemenschneider, J., van de Kamp, B., Monner, H.P., Cheung, R.C.M., Wales, C., and Cooper, J.E., Evaluation of a Compliant Droop-Nose 
Morphing Wing Tip via Experimental Tests. Journal of Aircraft, 2016. 54(2): p. $519-534$.

60. Vasista, S., Riemenschneider, J., Kamp, B.v.d., Monner, H.P., Cheung, R.C.M., Wales, C., and Cooper, J. Lessons learned from wind tunnel testing of a droop-nose morphing wingtip. Proceedings of SPIE Smart Structures and Materials + Nondestructive Evaluation and Health Monitoring, Vol. 9799, p. 9, Las Vegas, Nevada, USA, April 15, 206. SPIE.

61. TAMARACK. TAMARACK Aerospace Group. 2017, from: http://tamarackaero.com/.

62. Boeing 777x. Folding raked wingtip. 2017, from:

http://www.boeing.com/commercial/777x/\#/777-and-777x.

63. Aviation week. 777x Flight Deck and Wing Fold Controls Unveiled. 2017, from: http://aviationweek.com/new-civil-aircraft/777x-flight-deck-and-wing-fold-controlsunveiled.

64. Moholt, M. and Benafan, O., Spanwise Adaptive Wing. 2017, NASA Document ID: 20170009544: p. 17.

65. Ursache, N., Melin, T., Isikveren, A., and Friswell, M.I. Morphing Winglets for Aircraft Multi-Phase Improvement. 7th AIAA ATIO Conf, 2nd CEIAT Int'l Conf on Innov and Integr in Aero Sciences, 17th LTA Systems Tech Conf; followed by 2nd TEOS Forum. Belfast, Northern Ireland, UK, September 18-20, 2007, AIAA.

66. Ursache, N.M., Melin, T., Isikveren, A.T., and Friswell, M.I., Technology Integration for Active Poly-Morphing Winglets Development. 2008(43314): p. 775-782.

67. Smith, D.D., Isikveren, A.T., Ajaj, R.M., and Friswell, M.I. Multidisciplinary design optimization of an active nonplanar polymorphing wing. 27th Congress of the International Council of the Aeronautical Sciences. Nice, France September 19 - 24, 2010.

68. Smith, D.D., Ajaj, R.M., Isikveren, A.T., and Friswell, M.I., Multi-objective optimization for the multiphase design of active polymorphing wings. Journal of Aircraft, 2012. 49(4): p. 1153-1160. 
69. Ajaj, R.M., Friswell, M.I., Smith, D.D., and Isikveren, A.T., A conceptual wing-box weight estimation model for transport aircraft. The Aeronautical Journal, 2013. 117(1191): p. 533-551.

70. Smith, D.D., Lowenberg, M.H., Jones, D.P., and Friswell, M.I., Computational and Experimental Validation of the Active Morphing Wing. Journal of Aircraft, 2014: p. 1-13.

71. Cooper, J.E., Chekkal, I., Cheung, R.C.M., Wales, C., Allen, N.J., Lawson, S., Peace, A.J., Cook, R., Standen, P., Hancock, S.D., and Carossa, G.M., Design of a Morphing Wingtip. Journal of Aircraft, 2015. 52(5): p. 1394-1403.

72. Falcão, L., Gomes, A.A., and Suleman, A., Aero-structural Design Optimization of a Morphing Wingtip. Journal of Intelligent Material Systems and Structures, 2011. 22(10): p. 1113-1124.

73. Gomes, A.A., Falcao, L., and Suleman, A. Study of an articulated winglet mechanism. Collection of Technical Papers 54th AIAA/ASME/ASCE/AHS/ASC Structures, Structral Dynamics, and Materials Conference. Boston, Massachusetts, United States, April 8-11, 2013, AIAA.

74. Bourdin, P., Gatto, A., and Friswell, M.I. The Application of Variable Cant Angle Winglets for Morphing Aircraft Control. 24th AIAA Applied Aerodynamics Conference. San Francisco, California, United States, June 5-8, 2006, AIAA.

75. Bourdin, P., Gatto, A., and Friswell, M.I., Aircraft Control via Variable Cant-Angle Winglets. Journal of Aircraft, 2008. 45(2): p. 414-423.

76. Bourdin, P., Gatto, A., and Friswell, M.I. Potential of Articulated Split Wingtips for Morphing-Based Control of a Flying Wing. 25th AIAA Applied Aerodynamics Conference. Miami, Florida, United States, June 25-28, 2007, AIAA.

77. Gatto, A., Bourdin, P., and Friswell, M.I., Experimental Investigation into Articulated Winglet Effects on Flying Wing Surface Pressure Aerodynamics. Journal of Aircraft, 2010. 47(5): p. 1811-1815.

78. Castrichini, A., Siddaramaiah, V.H., Calderon, D.E., Cooper, J.E., Wilson, T., and Lemmens, Y., Preliminary investigation of use of flexible folding wing tips for static and dynamic load alleviation. The Aeronautical Journal, 2017. 121(1235): p. 
73-94.

79. Castrichini, A., Hodigere Siddaramaiah, V., Calderon, D.E., Cooper, J.E., Wilson, T., and Lemmens, Y., Nonlinear Folding Wing Tips for Gust Loads Alleviation. Journal of Aircraft, 2016. 53(5): p. 1391-1399.

80. Castrichini, A., Cooper, J.E., Wilson, T., Carrella, A., and Lemmens, Y., Nonlinear Negative Stiffness Wingtip Spring Device for Gust Loads Alleviation. Journal of Aircraft, 2016. 54(2): p. 627-641.

81. Cheung, R.C., Castrichini, A., Rezgui, D., Cooper, J.E., and Wilson, T. Testing of Wing-Tip Spring Device for Gust Loads Alleviation. 58th AIAA/ASCE/AHS/ASC Structures, Structural Dynamics, and Materials Conference. Grapevine, Texas, United States, January 9-13, 2017, AIAA.

82. Cooper, J., Miller, S., Sensburg, O., and Vio, G. Optimization of a Scaled Sensorcraft Model with Passive Gust Alleviation. 12th AIAA/ISSMO Multidisciplinary Analysis and Optimization Conference. Victoria, Bristish Columbia Canada, September 10-12, 2008, AIAA.

83. Miller, S., Cooper, J., and Vio, G. Development of an Adaptive Wing Tip Device. 50th AIAA/ASME/ASCE/AHS/ASC Structures, Structural Dynamics, and Materials Conference. Palm Springs, California, United States, May 4-7, 2009, AIAA.

84. Miller, S.J., Adaptive Wing Structures for Aeroelastic Drag Reduction and Load Alleviation. $\mathrm{PhD}$. School of Mechanical, Aerospace and Civil Engineering, University of Manchester, 2010.

85. Ricci, S., Castellani, M., and Romanelli, G., Multi-fidelity design of aeroelastic wing tip devices. Proceedings of the Institution of Mechanical Engineers, Part G: Journal of Aerospace Engineering, 2013. 227(10): p. 1596-1607.

86. Guo, S., Los Monteros, D., Espinosa, J., and Liu, Y., Gust Alleviation of a Large Aircraft with a Passive Twist Wingtip. Aerospace, 2015. 2(2): p. 135-154.

87. Han, M.-W., Rodrigue, H., Kim, H.-I., Song, S.-H., and Ahn, S.-H., Shape memory alloy/glass fiber woven composite for soft morphing winglets of unmanned aerial vehicles. Composite Structures, 2016. 140(Supplement C): p. 202-212.

88. Li, W., Xiong, K., Chen, H., Zhang, X., Su, Y., and Ren, Z., Research on 
variable cant angle winglets with shape memory alloy spring actuators. Acta Aeronautica et Astronautica Sinica, 2012. 33(1): p. 22-33.

89. Daniele, E., De Fenza, A., and Della Vecchia, P., Conceptual adaptive wing-tip design for pollution reductions. Journal of Intelligent Material Systems and Structures, 2012. 23(11): p. 1197-1212.

90. Jian, S., Hongliang, G., Fabrizio, S., Cristian, L., Yanju, L., and Jinsong, L., Active inflatable auxetic honeycomb structural concept for morphing wingtips. Smart Materials and Structures, 2014. 23(12): p. 125023.

91. Gatto, A., Mattioni, F., and Friswell, M.I., Experimental Investigation of Bistable Winglets to Enhance Aircraft Wing Lift Takeoff Capability. Journal of Aircraft, 2009. 46(2): p. 647-655.

92. Arrieta, A.F., Bilgen, O., Friswell, M.I., and Hagedorn, P., Passive load alleviation bi-stable morphing concept. AIP Advances, 2012. 2(3): p. 032118.

93. Saravanos, D., Arrieta, A.F., Bilgen, O., Friswell, M.I., and Hagedorn, P., Dynamic control for morphing of bi-stable composites. Journal of Intelligent Material Systems and Structures, 2012. 24(3): p. 266-273.

94. Kim, S.W., Lee, J.Y., and Cho, K.J. Towards a bistable morphing winglet for unmanned aerial vehicle(UAV). IEEE ISR 2013. Seoul, South Korea, October 24-26, 2013, IEEE.

95. Shelton, A., Tomar, A., Prasad, J., Smith, M., and Komerath, N., Active Multiple Winglets for Improved Unmanned-Aerial-Vehicle Performance. Journal of Aircraft, 2006. 43(1): p. 110-116.

96. Cerón-Muñoz, H.D. and Catalano, F.M., Experimental analysis of the aerodynamic characteristics adaptive of multi-winglets. Proceedings of the Institution of Mechanical Engineers, Part G: Journal of Aerospace Engineering, 2006. 220(3): p. 209-215.

97. Kauertz, S. and Neuwerth, G., Excitation of instabilities in the wake of an airfoil by means of active winglets. Aerospace Science and Technology, 2006. 10(7): p. $551-562$.

98. Breitsamter, C. and Allen, A., Transport Aircraft Wake Influenced by Oscillating 
Winglet Flaps. Journal of Aircraft, 2009. 46(1): p. 175-188.

99. Guha, T.K., Oates, W.S., and Kumar, R., Characterization of piezoelectric macrofiber composite actuated winglets. Smart Materials and Structures, 2015. 24(6): p. 065043.

100. Guha, T.K. and Kumar, R., Characteristics of a wingtip vortex from an oscillating winglet. Experiments in Fluids, 2016. 58(1): p. 8.

101. Brady, C. The Boeing 737 Technical Site. from: http://www.b737.org.uk/techspecsdetailed.htm.

102. UIUC Applied Aerodynamic Group. UIUC Airfoil Coordinates Database. from: http://m-selig.ae.illinois.edu/ads/coord_database.html.

103. Drela, M. and Youngren, H. AVL. 2017, from:

http://web.mit.edu/drela/Public/web/avl/.

104. Raymer, D.P., Aircraft Design: A conceptual Approach. Fourth ed. 2006, Virginia: American Institute of Aeronautics and Astronautics.

105. The MathWorks Inc., Matlab R2014b. 2014, The MathWorks Inc.

106. Mair, W.A. and Birdsall, D.L., Aircaft Performance. Cambridge Aerospace Series. 1996, Cambridge, UK: Cambridge University Press.

107. DaDeppo, D.A., Introduction to structural mechanics and analysis. 1999: Prentice Hall.

108. Dassault Systèmes, Abaqus 6.13 Analysis User's Guide. 2013, Dassault Systèmes.

109. Kaw, A.K., Mechanics of composite materials. Second ed. 2010, Boca Raton: CRC press.

110. Dayyani, I., Friswell, M.I., Ziaei-Rad, S., and Flores, E.I.S., Equivalent models of composite corrugated cores with elastomeric coatings for morphing structures. Composite Structures, 2013. 104: p. 281-292.

111. Xia, Y., Friswell, M.I., and Flores, E.I.S., Equivalent models of corrugated panels. International Journal of Solids and Structures, 2012. 49(13): p. 1453-1462.

112. Tsai, S.W. and Wu, E.M., A general theory of strength for anisotropic materials. Journal of composite materials, 1971. 5(1): p. 58-80. 
113. Jelaska, D.T., Gears and gear drives. 2012: John Wiley \& Sons.

114. GearWurx. Torxis Heavy Duty Linear and Rotary Servos. from: http://www.invenscience.com/index_files/torxis_rotary_servo.htm.

115. Samanta, A. and Mukhopadhyay, M., Finite element static and dynamic analyses of folded plates. Engineering Structures, 1999. 21(3): p. 277-287.

116. Mohammadi, H., Ziaei-Rad, S., and Dayyani, I., An equivalent model for trapezoidal corrugated cores based on homogenization method. Composite Structures, 2015. 131: p. 160-170.

117. Hosford, W.F., Mechanical Behavior of Materials. Second ed. 2010, Cambridge: Cambridge University Press

118. Markforged. The Mark Two. from: https://markforged.com/.

119. Vocke, R.D., Kothera, C.S., Woods, B.K.S., and Wereley, N.M., Development and Testing of a Span-Extending Morphing Wing. Journal of Intelligent Material Systems and Structures, 2011. 22(9): p. 879-890.

120. Olympio, K.R. and Gandhi, F., Optimal Cellular Core Topologies for One-Dimensional Morphing Aircraft Structures. Journal of Mechanical Design, 2012. 134(8): p. 081005-081005-10.

121. Dassault Systèmes. SOLIDWORKS. 2017, from:

http://www.solidworks.com/sw/3d-cad-design-software.htm.

122. SILEX LTD. from:

http://www.silex.co.uk/shop/superclear-silicone-sheet/superclear-silicone-sheet-40\% C2\%B0-shore-915mm-wide/c-24/c-105/p-689.

123. Permabond Engineering Adhesives. from: http://www.permabond.co.uk/cyanoacrylates.

124. Actuonix Motion Devices Inc. ACTUONIX P16-P Linear Actuator With FeedBack. from: https://www.actuonix.com/P16-P-Linear-Actuator-p/p16-p.htm.

125. Jewel B. Barlow, W.H.R., Alan Pope, Low-Speed Wind Tunnel Testing. Third ed. 1999: Wiley.

126. TVLM. Tornado VLM. 2010, from: http://tornado.redhammer.se/. 